\title{
What Makes an Electric Welding Arc Perform Its Required Functions
}

Thomas B. Correy

September 1982

Prepared for the U.S. Department of Energy under Contract DE-AC06-76RLO 1830

Pacific Northwest Laboratory Operated for the U.S. Department of Energy by Battelle Memorial Institute 


\section{DISCLAIMER}

This report was prepared as an account of work sponsored by an agency of the United States Government. Neither the United States Government nor any agency thereof, nor any of their employees, makes any warranty, express or implied, or assumes any legal liability or responsibility for the accuracy, completeness, or usefulness of any information, apparatus, product, or process disclosed, or represents that its use would not infringe privately owned rights. Reference herein to any specific commercial product, process, or service by trade name, trademark, manufacturer, or otherwise, does not necessarily constitute or imply its endorsement, recommendation, or favoring by the United States Government or any agency thereof. The views and opinions of authors expressed herein do not necessarily state or reflect those of the United States Government or any agency thereof.

\section{PACIFIC NORTHWEST LABORATORY operated by \\ BATTELLE for the \\ UNITED STATES DEPARTMENT OF ENERGY under Contract DE-AC06-76RLO 1830}

\begin{tabular}{|c|c|}
\hline \multicolumn{2}{|c|}{$\begin{array}{l}\text { Available from } \\
\text { National Technical Information Service } \\
\text { United States Department of Commerce } \\
5285 \text { Port Royal Road } \\
\text { Springfield. Virginia } 22151\end{array}$} \\
\hline \multicolumn{2}{|c|}{$\begin{array}{l}\text { NTIS Price Codes } \\
\text { Microfiche A01 }\end{array}$} \\
\hline \multicolumn{2}{|c|}{ Printed Copy } \\
\hline Pages & $\begin{array}{l}\text { Price } \\
\text { Codes }\end{array}$ \\
\hline $001-025$ & $\mathrm{~A} 02$ \\
\hline $026-050$ & $\mathrm{~A} 03$ \\
\hline 051.075 & $\mathrm{~A} 04$ \\
\hline $076-100$ & A05 \\
\hline 101.125 & A06 \\
\hline $126-150$ & $\mathrm{~A} 07$ \\
\hline $151-175$ & $A 08$ \\
\hline $176-200$ & $\mathrm{~A} 09$ \\
\hline $201-225$ & $A 010$ \\
\hline $226-250$ & A011 \\
\hline 251.275 & A012 \\
\hline $276 \cdot 300$ & $\mathrm{~A} 013$ \\
\hline
\end{tabular}


WHAT MAKES AN ELECTRIC WELDING ARC PERFORM ITS REQUIRED FUNCTION

Thomas B. Correy

September 1982

Prepared for the U.S. Department of Energy under Contract DE-AC06-76RLO 1830

Pacific Northwest Laboratory Richland, Washington 99352 



\section{FOREWORD}

This report is based on work performed at the Pacific Northwest Laboratory, operated by Battelle Memorial Institute for the U.S. Department of Energy, by Thomas B. Correy, Senior Development Engineer, Materials Department of Pacific Northwest Laboratory.

It was presented orally at the IEEE sponsored session, American Welding Society Meeting at Detroit, Michigan, April 24-28, 1967. Upon review for publication, the decision was made to enlarge its contents to include Heat Transfer by Electric Arcs, the Effect of Pressure on the Inert Gas Shielded Welding Arc, Pulsed Arc Welding, Procurement of Welding Power Supplies, and Maintenance of Welding Power Supplies.

This paper was written in the form of an abstract to provide the greatest amount of information on the welding arc's performance with the minimum amount of reading. For those who need or want details of any function, a broad reference list, a supplementary reference list, and an appendix are included.

During the preparation of this paper many significant developments have been made in arc welding processes. Many of these have been incorporated in the text. Near the end of the preparation it was no longer feasible to incorporate them into the text due to the amount of rewriting required. Therefore, items appearing in the literature and of interest related to the theme of the text have been included as addenda. 


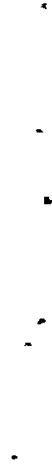




\section{$\underline{\text { ABSTRACT }}$}

The welding arc is a driven circuit element, and its performance in welding is determined by the instantaneous value and the variation of the instantaneous value of the force driving it. It is a very short point-toplane arc surrounded by an atmosphere whose pressure may be any place from a few pounds per square inch absolute to at least 600 psia and is further complicated by non-uniform electric and magnetic fields, reversing non-linear volt-ampere characteristic, filler metal, if used, and shielding gas(es). The arc's performance is further complicated by very high de-ionization and thermal electron emission decay rates as the current drops to a low value or is interrupted. Conversely, the rate of rise of current is limited by the rates of rise of ionization and thermal electron emission. In alternatingcurrent welding, arc performance is further complicated by polarity reversal and the sudden requirement to establish a new cathode spot at its operating temperature.

Direct-current constant current welding power supplies available vary from the three-phase, full-wave, rectified units through the six-phase, full-wave, rectified units whose output current on a single range has a ripple content at any value of current that will not exceed the commutator ripple of a motor generator. The basic test of a fine quality directcurrent welding power supply is: Can the welding current be varied from maximum to minimum or minimum to maximum while welding aluminum with helium as a shielding gas at maximum welding speed that will produce a stable arc at maximum output current without the arc going out, becoming unstable, or squealing? The benefits derived from these power supplies as you go from the various three-phase full-wave units through the six-phase units are:

- Positive, smoother, faster arc starting

- Higher welding speeds

- Less porosity

- Smoother welds with minimum heat input

- Reduced quantity of shielding gas 
Pulsed current direct-current welding power supplies are available from the general purpose units whose welding current rise and decay times are exponential and are fixed by the relations of the resistance, inductance, and capacitance of the welding current-carrying circuit to the sophisticated units that produce virtually a square wave with current rise and decay times of $10^{7} \mathrm{~A} / \mathrm{sec}$. In addition, they are available with a variable high frequency current superimposed on both the high and low pulse currents. These units can be obtained with all of the sophisticated programming and controls available on the regular sophisticated direct-current welding power supplies. Pulsed direct-current power supplies can be operated as a continuous direct-current power supply.

Alternating-current welding power supplies available vary from the nonbalanced-current, saturable reactor controlled units requiring continuous high-frequency, to the balanced sine wave units*, to balanced current square waves that have equal time periods and virtually constant amplitude at all instants for each half cycle and the tailored pulsed current square wave. The benefits derived from these power supplies as you go from a saturable reactor control with its badly distorted third harmonic sine wave through the pulsed square wave are:

- Smoother and faster arc starting with no continuous high frequency.

- Higher welding speeds, less porosity, and smoother welds with min imum heat input.

- Retention of cathodic etching that produces cleaner welds while welding with virtually direct-current.

- A stable arc when welding deep narrow grooves with submerged arc welding when square wave alternating-current is used.

*For a detailed discussion of the effects and correction of unbalanced alternating welding current, see References 13 and 14 in the Supplementary References. 


\section{ACKNOWLEDGMENT}

I wish to acknowledge the contributions of all the people who have assisted with this paper during its twelve years of preparation. So many have made suggestions, contributed additions, and reviewed it that it is impossible to recognize them individually. Thus, to each and everyone who has in any way assisted, my heartfelt thanks. 


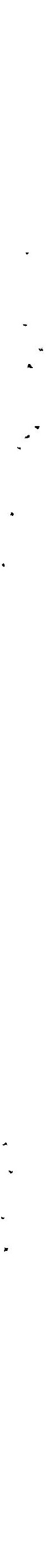


FOREWORD. . . . . . . . . . . . . . . . . . . . . .

ABSTRACT. . . . . . . . . . . . . . . . . . V v

ACKNOWLEDGMENT. . . . . . . . . . . . . . . . . . . . vii

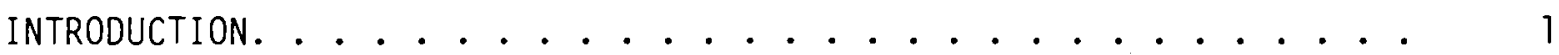

PHYSICS OF THE WELDING ARC.................... 3

Cathodes and Anodes. . . . . . . . . . . . . . . 3

Direct-Current Welding Arcs. . . . . . . . . . . 7

Modes of Operation. . . . . . . . . . . . . 21

Variable Weld Penetration ............. 23

Arc Ignition Characteristics. . . . . . . . . . . 26

Principle of Similarity.............. . . 27

Pole Spots.................. . . 28

Effect of Electrode Geometry. . . . . . . . . . . 29

Mixed and Contaminating Gases ............ . 31

Magnetic Arc Blow . . . . . . . . . . . . 32

Phenomena of Travel Speed and Arc Stability . . . . . . 36

Pressurizing Above Atmospheric Pressure . . . . . . . . 43

Alternating Current Welding Arcs ............. 47

Influence on Arc Characteristics. .......... . 47

Sinusoidal Current Arc Characteristics. . . . . . . . 47

Half Cycle Polarity Reversal. . . . . . . . . . 50

Arc Ignition Characteristics. ............ 51

Arc Hysteresis. . . . . . . . . . . . . 53

Effect of Cathode Substances on Restriking. . ...... 55

Effect of Travel Speed on Arc Stability.......... 55

HEAT TRANSFER OF DIRECT-CURRENT WELDING ARCS. . . . . . . . . . 57

Heat Transfer Within Arc . . . . . . . . . . . 57

Transport Properties Within Arc ........... 57

Plasma Composition. .............. 57 
Plasma Energy Transfer to a Solid Surface. . . . . . . . . 69

Plasma Heat Transfer Without Current Flow to an Anode . . . 69

Plasma Heat Transfer With Current Flow to an Anode. . . . . 75

Free Burning Arcs With Plane Anodes . . . . . . . . 77

Cathode Axis Parallel to a Plane Anode. . . . . . . . 80

Cylinder Geometry With an Annular Anode . . . . . . . . 89

Heat Flow Appendix. . . . . . . . . . . . . 91

Energy Flux Relationships. . . . . . . . . 91

Temperature Field Created by a Stationary Heat Source. 93

List of Symbols. . . . . . . . . . . . . . 94

Plasma Energy Transfer to a Molten Surface . . . . . . . 9 96

Plasma Heat Transfer With Current Flow. . . . . . . . 96

WELDING POWER SUPPLIES. . . . . . . . . . . . 100

Welding Power Supply Characteristics .......... 100

Direct-Current Welding Power Supplies ......... 100

Pulsed Current Direct-Current Welding Power Supplies. . . . 110

Alternating-Current Welding Power Supplies. . . . . . 122

PROCUREMENT .............................. 139

Constant Current Power Supplies. . . . . . . . . . . . 139

Pulsed Current Power Supplies. . . . . . . . . . . . . 140

Specifications ................. 140

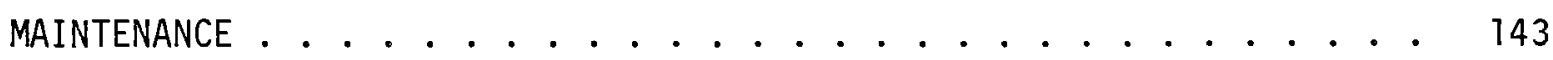

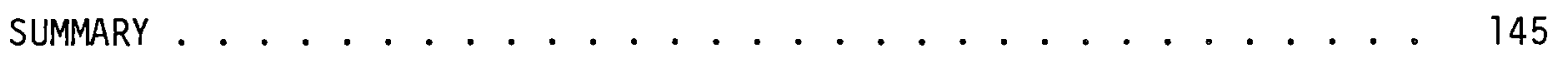

REFERENCES. . . . . . . . . . . . . . . . . . . . . . 149

SUPPLEMENTAL REFERENCES ...................... 159

APPENDIX A - THE GLOW DISCHARGE AND ELECTRIC ARC COMPONENTS . . . . . 163

APPENDIX B - COMPOSITION OF INCONEL ALLOY $600 \ldots \ldots 7$

APPENDIX $C$ - COMPOSITIONS OF ZIRCALLOYS-2 AND -4. . . . . . . 169

APPENDIX D - ALTERNATING-CURRENT BALANCING. . . . . . . . 171

APPENDIX E - NATURAL IONIZATION IN AIR. . . . . . . . . 173

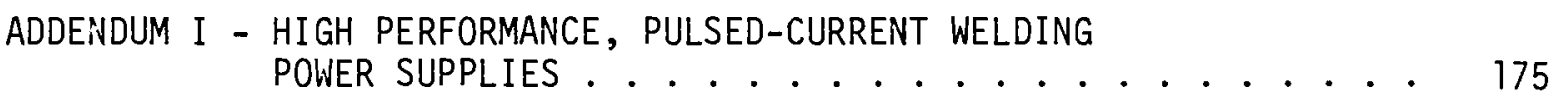

Introduction . . . . . . . . . . . . 175

Current Ripple and Current Pulsing .......... 177 
Pulse Formation of Direct-Current. . . . . . . . . . 177

Pulse Shape Synthesizing.............. 178

Certification of High Performance Welding Power Supplies . . . 179

ADDENDUM II - ELECTRICAL EFFICIENCIES OF WELDING POWER SUPPLIES. . . . . . . . . . . 187

ADDEMDUM III - SQUARE WAVE ALTERNATING-CURRENT NARROW GROOVE SUBMERGED ARC WELDING. . . . . . 189

DISTRIBUTION. . . . . . . . . . . . . . . . . . 193 


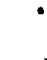




\section{FIGURES}

1 Representative Burning Voltage Distribution for an 8 Volt, 100 Ampere Arc With 1/16 inch Thoriated Tungsten Cathode,

Zircaloy Anode, Argon Shielding Gas. .......... 6

2 Volt-Ampere Characteristics of Arcs in Argon, Arc Length $=0.1$ inch $(2.54 \mathrm{~mm}) \ldots \ldots \ldots 7$

3 Volt-Ampere Characteristics of High Current Arcs . . . . . . 8

4A Voltage-Current Characteristics of the Arc in Helium for Electrode Separations of 0.1 inch $(2.54 \mathrm{~mm})$ and 0.3 inch

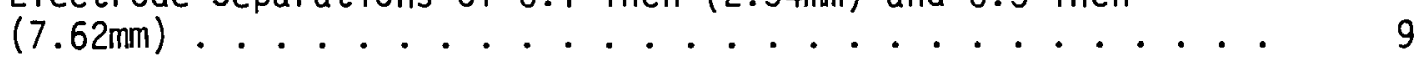

4B Same as Figure 4A But for Separations of 0.05 inch $(1.27 \mathrm{~mm})$, 0.2 inch $(5.08 \mathrm{~mm})$ and 0.4 inch $(10.16 \mathrm{~mm}) \ldots . . . .99$

5 Arc Voltage as a Function of the Electrode Separation. . . . . 10

6 Voltage Gradient as a Function of the Mixture for $\mathrm{I}=50$ Amperes and for Electrode Separations Between

0.1 inch $(2.54 \mathrm{~mm})$ and 0.3 inch $(7.62 \mathrm{~mm}) . \ldots . . .$.

$7 A$ Arc Voltage as a Function of the Mixture for I - 20 Amperes and I = 60 Amperes Electrode Separation, etc. . 12

7B Same as 7A But for a Separation of 0.4 inch $(10.16 \mathrm{~mm})$. . . . 12

8 Arc Voltage-Arc Length Relationship for 3 Inert Gases and One Mixture................. 13

9A Sketch of Arcs Composition With Corresponding Photograph Electrode Negative.............. 14

9B Sketch of Arcs Composition With Corresponding Photograph Electrode Positive . . . . . . . . . . . . .

10 Approximate Behavior of Sputtering Yield With Incident Ion Energy for Argon and Helium Ions Normally Incident Upon Aluminum. . . . . . . . . . . . . . . 17

11 Electron Micrographs of Cathodically Etched Zircaloy-2 . . . 19

12 Power Delivered to the Anode-Arc Current Relationship. . . . . 20

13 Schematic Representation of Five Types of the Argon Tungsten Arc ............... 22

14A Total Thermal Conductivity of Some Representative Gases as a Function of Temperatures. . . . . . . . . . 24

14B Electrical Conductivities of Argon, Nitrogen, and Hydrogen as a Function of Temperature . . . . . . . . . . . .

15 Effect on Arc Voltage of Different Metals for Anode and Cathode. 


\section{FIGURES (cont'd.)}

16A Cathodically Etched Tracks Produced by Instability of Arc Cathode Spot During the First Few Milliseconds of Ignition in Argon. . . . . . . . . . . . . . . . . . . . 27

16B Helium Produces No Etching Though Instability Was Observed. . . 27

17 Effect of Vertex Angle of Conical Tip on Depth of Penetration. . . . . . . . . . . . . . . . 30

18 Effect of Vertex Angle of Conical Tip on Weld Width . . . . . . 30

19 Erosion of Tungsten Electrode by Water Vapor in the Shielding Gas..................... . 31

20 A Magnetic Field Around a Conductor . . . . . . . . . . . 33

20B Magnetic Field of a Conductor in Interaction with an Outside Magnetic Field. .............. . 33

21A Magnetic Field of an Arc in the Proximity of Its Return Connection. . . . . . . . . . . . . . . . 34

$21 \mathrm{~B}$ Magnetic Field in the Case of the Balanced Return Connection .................. . . 34

22 Assymetric Location of Eddy Currents Caused by Heated Non-Magnetic Area.................... 35

23 Arc Formation and Extension . . . . . . . . . . . . 37

24 Anode Jet.................... . . . . 38

25 Variation of Anode Spot Characteristics With Electrode Speed .................... 38

26 Variation of Anode Spot Characteristics With Current. . . . . . 39

27 Variation of Arc Characteristics With Electrode Separation. . . 40

28 Variation of Area of Individual Anode Spots With Total Arc Current ...................... . 40

29 Variation of Total Lateral Area Melted Per Second With Speed of the Moving Tape ............... 41

30 Variation of the Area of the Individual Anode Spots With Speed of the Moving Tape . . . . . . . . . . . . 42

31 Rate of Growth of Lateral Area of Individual Anode Spots. . . . 42

32 Variation of Apparent Current Density With Electrode Speed. . . 43

33 Variation of Apparent Current Density With Total Arc Current. . 44

34 Electrode Spacing-Arc Voltage Characteristics . . . . . . . 45

35 Gas Pressure-Arc Voltage Characteristics. . . . . . . . . . . 45

36 Voltage-Current Relationships for 200 Ampere Balanced Sine Wave Inert Arc. . . . . . . . . . . . . . 48 


\section{FIGURES (cont'd.)}

37 Sequence of Alternating-Current Arc Starting Sequence, Damage Done to Electrode by Arc Striking Shank, and Cathodic Etching of Shank. . . . . . . . . . .

38 Volt-Ampere Characteristics of Alternating-Current Balanced Sine Wave Argon Arc . . . . . . . . . . .

39 Arc Hysteresis Loop for 200 Ampere Balanced Sine Wave Inert Arc. . . . . . . . . . . . . . . 54

40 Dissociation in Molecular Gases at 1 atm Pressure. . . . . . 58

41 Argon-Shielded Arc Plasma Composition. .......... 58

42 Nitrogen-Shielded-Arc Plasma Composition . . . . . . . 59

43 Hel ium-Shielded Arc Plasma Composition .......... 59

44 The Dependence of Arc-Plasma Shape on Composition

(Percent by Volume) of Argon-Helium Gas-Shielded Arcs. . . . . 63

45 Dependence of Plasma Gradient on Composition (Percent by Volume) of Argon-Helium Shielded Arcs. . . . . . . 64

46 Temperature Distribution in an Argon Shielded Tungsten Arc 300 Amperes Electrode Negative............ 64

47 Opposing Pressure of Self Magnetic Compression and Kinetic Gas Pressures in Arcs. . . . . . . . . . . . 67

48 PRANDTL Number for Frozen and Equilibrium Composition. . . . . 72

49 Heat Transfer Parameter Determined Analytically and Experimentally as a Function of Gas Enthalpy ........ 73

50 Profiles of Gas Temperature $T_{a}$ and Electron Temperature $T_{e}$ in a Wall Stabilized Arc.............. 74

51 Energy Balance of Anode Surface Element. . . . . . . . . . 76

52 Radial Distribution of Energy Transfer at Anode Surface. . . . 79

53 Impinging Jets ................... 81

54 Temperature Field Created by a Moving Point Heat Source On the Surface of a Semi-Infinite Body ......... 83

55 Distribution of Energy Transfer at Anode Surface, Argon. . . . 84

56 Time Dependence of the Current Distribution for the Segmented Anode. ................ 85

57 Frames From High Speed Movies Demonstrating Fluctuating Mode in Argon. . . . . . . . . . . . . . 86

58 Steady and Fluctuating Mode of an Arc. . . . . . . . 87

59 Schematic Drawing of Water-Cooled Cascaded Arc, Diagonal Hatching - Conducting Material, Solid Black Electrical Insulator. . . . . . . . . . . . . . . 


\section{FIGURES (cont'd.)}

60A Fraction of Total Heat Transferred to Anode Shown in Figure 59 by Indicated Transfer Mechanisms. . . . . . . . . . 92

60B Fraction of Total Heat Transferred to Anode Shown in Figure 59 by Indicated Transfer Mechanisms. . . . . . . . . . . 92

61 Copper: Relationship Between Heat Input. . . . . . . . . 96

62 Variation of Positive Column Potential Gradient With Arc Current. . . . . . . . . . . . . . . . 99

63 Ripple in the Full-Wave Current of 2-, 3-,6-, and 12-Pulse Rectifiers................ 101

64 Definition of Current Ripple................ 103

65 Oscillograms of Three-Phase Half-Wave Magnetic Amplifier Showing Amount of Ripple. . . . . . . . . . . . . 104

66 Oscillograms of Three-Phase Full-Wave Magnetic Amplifier Showing Amount of Ripple. . . . . . . . . . . . . . 105

67 Oscillograms of Three-Phase Full-Wave Magnetic Amplifier Showing Amount of Ripple................. . 106

68 Oscillograms of Three-Phase Full-Wave Magnetic Amplifier Rectifier Showing Amount of Ripple. . . . . . . . . . . 106

69 Oscillograms of Six-Phase Half-Wave Silicon Controlled Rectifier Showing Amount of Ripple. . . . . . . . . . 108

70 Schematic of an Ideal Pulsed Current Square Wave Form and Associated Pulse Parameters . . . . . . . . . . . . . 111

71 A Representative General Purpose Pulsed Current Square Wave Form and Its Three Phase $60 \mathrm{~Hz}$ Ripple............ 113

72 Hybrid Current Pulse. . . . . . . . . . . . . . . . 114

73 Relationship Between Pulse Current Amplitude and Duration For Full Penetration on $3.5 \mathrm{~mm}$ (0.14 in.) Stainless Steel. . . . 115

74 Top width of Weld Beads for Full Penetration on $3.5 \mathrm{~mm}$ (0.14 in.) Stainless Steel................. 117

75 Range of Current Wave Shapes Investigated . . . . . . . . . 124

76 Saturable Reactor 80-Volt Open-Circuit Un-Balanced Current High-Frequency Stabilized Third Harmonic Distorted Sine Wave. . 124

77 Saturable Reactor 165 Volt Open-Circuit Balanced Current No Stabilization Third Harmonic Distorted Sine Wave . . . . . .

78 Moving Coil Transformer 0scillograms of Arc Starting Sequences for 100 Volts Open-Circuit Sine Wave Current With No Stabilization or Balancing. . . . . . . . . . . . . . . . 


\section{FIGURES (cont'd.)}

79 Moving Coil Transformer Oscillograms of Arc Starting, Welding, and Crater Filling Sequences for 100 Volt

Open-Circuit Balanced Sine Wave Current Arc,

No Stabilization. . . . . . . . . . . . . . . . .

80 Moving Coil Transformer Oscillograms of Arc Starting, Welding, and Crater Filling Sequences for 150 Volt Open-Circuit Sine Wave With No Stabilization or Balancing . .

81 Moving Coil Transformer Oscillograms of $150 \mathrm{Volt}$ Open-Circuit Balanced Sine Wave Arc, No Stabilization . . .

82 Sel in Saturable Reactor Oscillograms of 165 Volts Open-Circuit Balanced Cube Root Sine Wave Arc, No Stabilization.

84 Weed Saturable Reactor With Inductance. . . . . . . . . . 132

85 Pulsed Square Wave Alternating-Current. . . . . . . . . 133

86 Wide Ranges, Balanced Sine Wave Current . . . . . . . . . 136

87 Characteristics of the Low Pressure Glow Discharge. . . . . . 164

88 Steady State Welding Current. . . . . . . . . . . . . 180

89 Square Wave Slow Pulsing. ............... 180

90 Square Wave Fast Pulse. . . . . . . . . . . . . 181

91 Slow Sawtooth Pulsing............... 181

92 Fast Triangular Pulsing . . . . . . . . . . . . 182

93 Pulse With Upslope. . . . . . . . . . . . . . . . . 182

94 Linear Sloped Penetration Peak Pulse. . . . . . . . . . 183

95 Penetration Peak Pulse With Peak Hold . . . . . . . . . 183

96 Linear Sloped Penetration Peak Pulse With Hold and Down Slope With Pulsing . . . . . . . . . . . . 184

97 Square Current Pulses With Sawtooth Pulses Superimposed on Peak and Background Pulses . . . . . . . . . . . . 184

98 Square Current Pulses With Sine Wave Pulses Superimposed on Peak and Background Pulses. . . . . . . . . .

99 Square Current Pulse With Square Pulses Superimposed on Peak and Background Pulses . . . . . . . . . . . . . . .

100 Square Current Pulses With Sine Wave Pulses Superimposed on Peak and Background Pulses . . . . . . . . . . . . . 


\section{FIGURES (cont'd.)}

101 Square Current Pulses With Sine Wave of Ten Times the Frequency of Figure 100 Superimposed on Peak and Background Pulses Showing the Building Block Pulse of One Millisecond Duration Used to Tailor the Pulses. . . . . . . . . .

102 Comparison of Sine and Square Wave Current Produced With Alternating-Currents Submerged Arc Welding. . . . . . . .

103 Comparison of Submerged Arc Welding Grooves for Sine and Square Wave Welding Currents for the Same Material and

Thickness....................... 


\section{TABLES}

1 Observed Number of Cathode Spots for Different Electrode Materials. . . . . . . . . . . . . . . 4

2 Square Current Wave, Arc Stability Relationships For Variable Gas Ratios. . . . . . . . . . . . . . . 56

3 Dependency of Temperature Parameter Ratio on the Dimensionless Parameter $\mathrm{Pe}_{r}$. . . . . . . . . . . . . 83

4 Values of Constants in the Heat-Input Equation . . . . . . . 97 


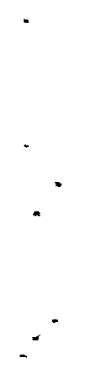


WHAT MAKES A WELDING ARC PERFORM ITS REQUIRED FUNCTION

\section{INTRODUCTION}

The work that can be obtained from a welding arc is determined by the static and dynamic characteristics of the system through which the power to energize it flows.

The welding arc is distinguished as a low-voltage high-current discharge that is non-linear with reversing volt-ampere characteristics as the welding current changes from minimum to maximum and maximum to minimum. A stable arc requires the establishment and maintenance of anode (positive) and cathode (negative) spots with the cathode spot exerting the greatest influence as the electrons, which carry the main portion of the current, originate at the cathode. For a finite electrode material and diameter at medium to high current values, the electron current is dependent on ion bombardment of the cathode spot and resistance heating in the electrode starting at the surface of the cathode spots. For most electrode materials, above ten amperes, there are multiple cathode spots. At low values of current for a high current cathode, there is not sufficient heat at the cathode nor in the plasma column to supply all of the required electrons by thermal emission. The mechanism of field emission, pulling the electrons from the cathode surface with the electrostatic field produced by the arc voltage, starts supplying electrons as the current decreases, requiring a rising arc voltage.

Generally speaking, the higher the resistivity and the lower the thermal conductivity of a metal, the easier it is to establish a stable thermionic cathode. This is demonstrated by the ease with which an alternating-current arc attaches itself to a very small piece of tungsten fused to the surface of aluminum in preference to establishing a cathode spot on the aluminum.

At any instant there must be adequate voltage available to supply the burning voltage of the arc at its required current or the arc will be unstable or go out. 
The static and dynamic characteristics of the arc and the system through which the power flows to energize the arc influence the starting and burning characteristics of the welding arc. In addition, these same characteristics determine the maximum current programming rates and minimum current iimits.

Extensive work has been performed at Hanford to determine the most desirable characteristics for welding power supplies for precision gas tungsten arc welding. From the results of this work and work of others, there are now available welding power supplies to satisfy the most sophisticated gas tungsten arc welding requirements. 


\section{PHYSICS OF THE WELDING ARC*}

A. CATHODES AND ANODES**

The electric arc as used in welding has the following characteristics:

- A low to high-pressure, high-current, low-voltage electrical discharge in gases not 1 imited to atmospheric pressure.

- Has multiple cathode spots above approximately ten amperes.

- Current density in the cathode spot ranging from $10^{4}$ to $10^{7} \mathrm{~A} / \mathrm{cm}^{2}$ $\left(6.45 \times 10^{4}\right.$ to $\left.6.45 \times 10^{7} \mathrm{~A} / \mathrm{in} .^{2}\right){ }^{1,2,3}$

- A cathode fall potential in the order of the minimum ionization potential of the gas or metal vapor, about 10 volts. ${ }^{4}$

- An anode fall potential that may be from 1 volt to over 35 volts. ${ }^{5,6}$

- Current densities in the anode spot ranging from 100 to $2500 \mathrm{~A} / \mathrm{cm}^{2}$ (645 to $16,125 \mathrm{~A} /$ in. $^{2}$ ).

- An arc potential in the order of 8 to 40 volts.

In addition, the light from the plasma contains spectral lines from the anode and cathode materials and shielding media. For most cathode materials and for currents in excess of 10 amperes, the cathode spot becomes multiple spots. ${ }^{7}$ In Table 1 are shown the number of cathode spots observed for various cathode materials and currents. ${ }^{8}$

High speed motion pictures of high current arcs between a variety of materials show average cathode spot currents ranging from 6 to 26 amperes. ${ }^{9}$ The diameters of the cathode spots for a low current arc with a single cathode spot may vary from approximately $1 \times 10^{-3} \mathrm{~cm}$ to $4 \times 10^{-3} \mathrm{~cm}$ (0.0394 to 0.01567 in.)..$^{10}$ The time to establish a single cathode spot is

\footnotetext{
*For a discussion of arc physical structure, see Appendix A. ** In the following discussion the theory of the arc mechanism is 1 imited to those factors that are directly related to welding. For example, the usefulness of the discussion is not affected regardless of whether one electron leaving the cathode reaches or does not reach the anode, whether any one positive ion travels the full length of the arc, or whether the ions have single or multiple ionization.
} 
TABLE 1. Observed Number of Cathode Spots for Different Electrode Materials

OBSERVED NUMBER OF CATHODE SPOTS FOR DIFFERENT ELECTRODE MATERIALS

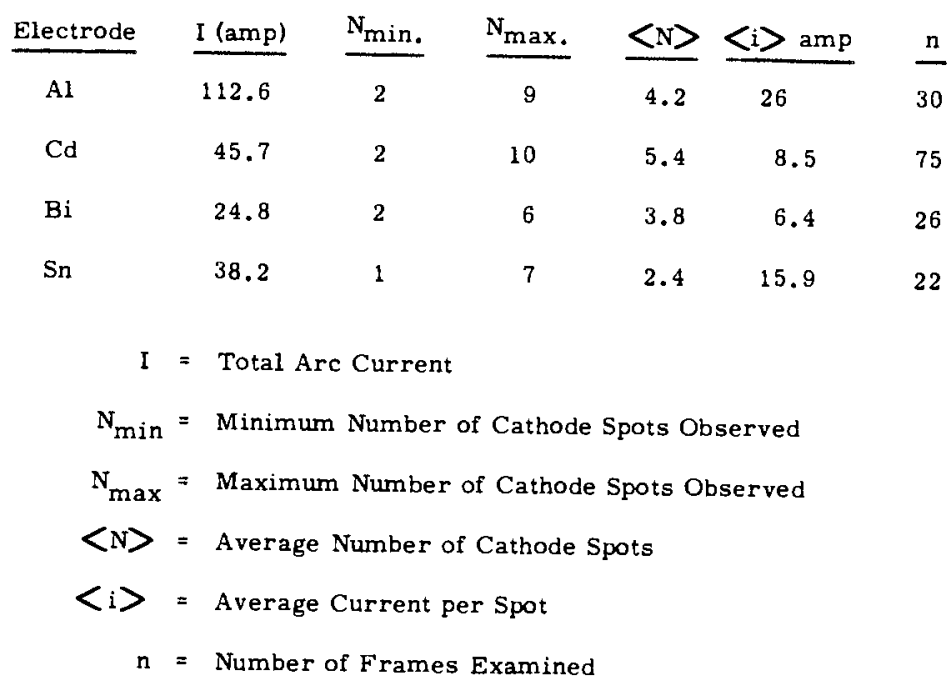

dependent on the current density, thermo-electron emission properties, resistivity, thermal conductivity, and the melting and boiling points of the cathode material. It may vary from a few microseconds to a few milliseconds. 11

When initiated between parallel plate electrodes, the arc has a constant voltage with changing current. This occurs as long as the ionized plasma does not exceed the paraliel surfaces of the electrodes or become less than that required for adequate thermal electron emission from the cathode. If the plasma volume increases beyond the parallel electrode surfaces, or decreases below the point of adequate thermal electron emission, the voltage will start to rise.

The cathode is at the end of the negative electrode which is the point of electron current transfer from the metal electrode to the plasma. The cathode is the most important region in the arc as its size, contour, and 
symmetry determine penetration and weld symmetry. It is the source of the electrons which carry some 90 percent of the current. The remainder of the arc current is carried by positive ions which move toward the negative terminal or cathode and proceed through the cathode fall space to develop the current continuity at the cathode terminal. ${ }^{12}$ Though the equivalent of many lifetimes has been spent studying it, the mechanics of electron emission from the arc cathode spot are still rather poorly understood. However, the thermal electron emission mechanism is well understood for such metals as tungsten, whose cathodes have relatively low current densities. A positive ion cloud surrounds the cathode. Positive ion bombardment from this cloud raises a spot on the cathode to the thermal electron emission temperature needed to supply the required electron currents. These ions are drawn from the cloud surrounding the cathode by the negative potential of the cathode. The low boiling point materials, such as aluminum, are characterized by high cathode current densities. These cannot produce the required electrons by thermionic emission unless the cathode spot is more than twice the boiling point of the metal, about $5000^{\circ} \mathrm{K}\left(9000^{\circ} \mathrm{F}\right)$. The existence of a surface so far above the boiling point of the material has not been established. 13

The cathode spot phenomenon at low currents appears to be definitely associated with the presence of oxide layers or similar films on the electrode surface. It has thus far been impossible to maintain a stable arc cathode spot on low boiling point metals in an inert gas atmosphere unless some oxide is present. This has also been observed for thin sheet tungsten cathodes where cooling is so great that the spot does not produce an incandescent area. The arc could not be maintained in argon or helium uniess the surface of the tungsten was slightly oxidized. ${ }^{14}$

The anode of an arc may be at any temperature below its elevated equilibrium temperature without affecting its stability. For instance, an arc will be stable on a water-cooled block with no evidence of melting in the anode spot. ${ }^{15}$ This principle is employed in mercury arc rectifier anodes. 
The burning voltage of a welding arc is divided into three distinct regions (Figure 1). The figure proportions are those of a direct-current welding arc. 16 A considerable amount of voltage is used up in a relatively short distance in front of the cathode. This distance is known as the cathode-drop region. The cathode-drop region is one of very high positiveion space charge. In an arc at atmospheric pressure or above, a cathode dark space has never been observed; its thickness therefore must be extremely smal1. The positive column is a region of uniform longitudinal voltage gradient whose magnitude depends on the shielding gas, gas pressure, and arc current. The total voltage along the positive column is a linear function of the length of the column. The anode-fall region uses up a considerable portion of the arc voltage in a relatively short distance in front of the anode. In addition, the magnitudes of each of these voltages is affected by the compositions of the electrodes and shielding gas. At the temperature of the welding arc, the metal vapors from each electrode migrate through the plasma. The metal vapors may influence the plasma and the opposite electrode drop. 17

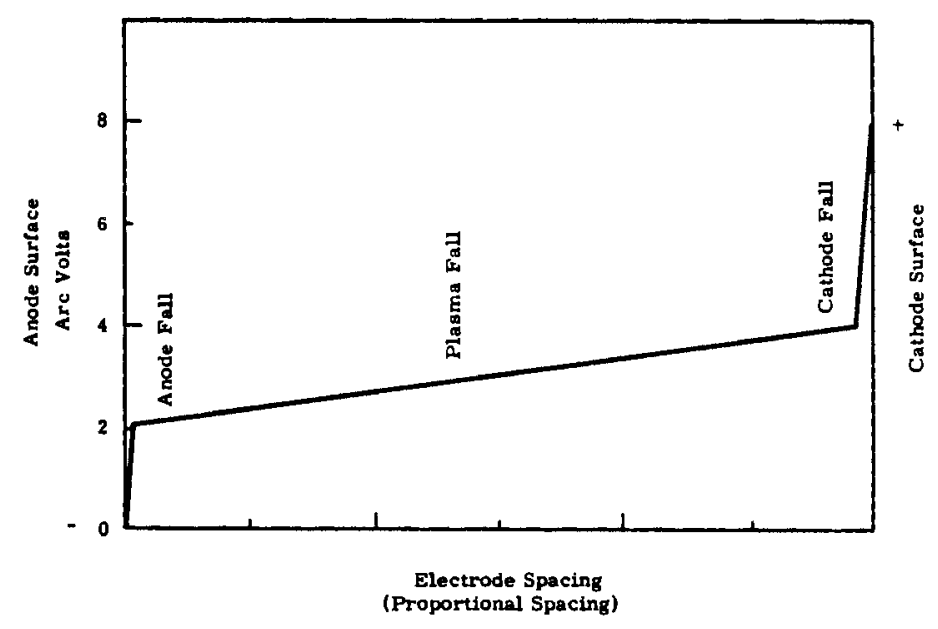

FIGURE 1. Representative Burning Voltage Distribution for an 8 Volt, 100 Ampere Arc With 1/16 inch Thoriated Tungsten Cathode, Zircaloy Anode, Argon Shielding Gas 
The mininum theoretical voltage for any arc is the sum of its anode and cathode drops. These voltages must be available before any arc can be struck or exist.

\section{B. DIRECT-CURRENT WELDING ARC}

The welding arc is distinguished as a low-voltage high-current discharge that is non-linear and has reversing volt-ampere characteristics (Figure 2). 18 In the low current range, the drooping characteristic is caused by insufficient heat to supply the thermal electrons. As the current increases, a region is reached at which the arc voltage remains essentially constant, thus producing a linear change of heating as the welding current changes in the current-electrode size welding range. In this portion, there are adequate thermal electrons as the plasma cross section in contact with the electrode increases until it covers the end of the electrode. With further increase in current, the plasma starts moving over the edge of the electrode (anode or cathode) and the voltage starts to rise. At low values of current

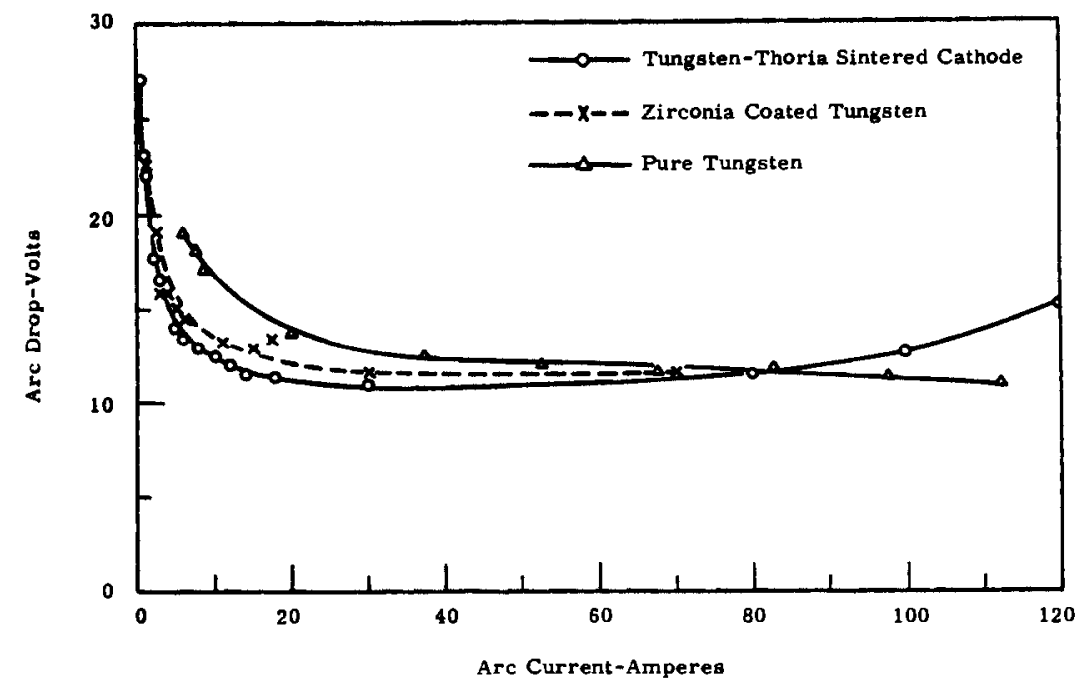

FIGURE 2. Volt-Ampere Characteristics of Arcs In Argon Arc Length $=0.1$ inch $(2.54 \mathrm{~mm}) 18$ 
for a high current rated cathode there is not sufficient heat at the cathode to supply all of the electrons by thermal emission so the mechanism of field emission starts supplying electrons. Generally speaking, the higher the resistivity and the lower the thermal conductivity of a metal, the easier it is to establish a stable thermionic cathode. This is demonstrated by the ease with which an alternating-current arc attaches itself to a very small piece of tungsten fused to the surface of aluminum.

Argon produces a stable arc over the full current range (Figure 2). ${ }^{18}$ Helium has a bi-stable region (Figures $3,{ }^{19} 4 \mathrm{~A}$, and $4 \mathrm{~B}^{20}$ ).

There is a decline in voltage with corresponding increases in current in the helium arc up to the transition points where the voltages take a sudden drop. Transitions take place spontaneously and may be in either direction. The jump from the upper curve to the lower curve, with a particular set of parameters, is random and does not occur at any definable value of current. It may be an abrupt jump or it may be a gradual change. The

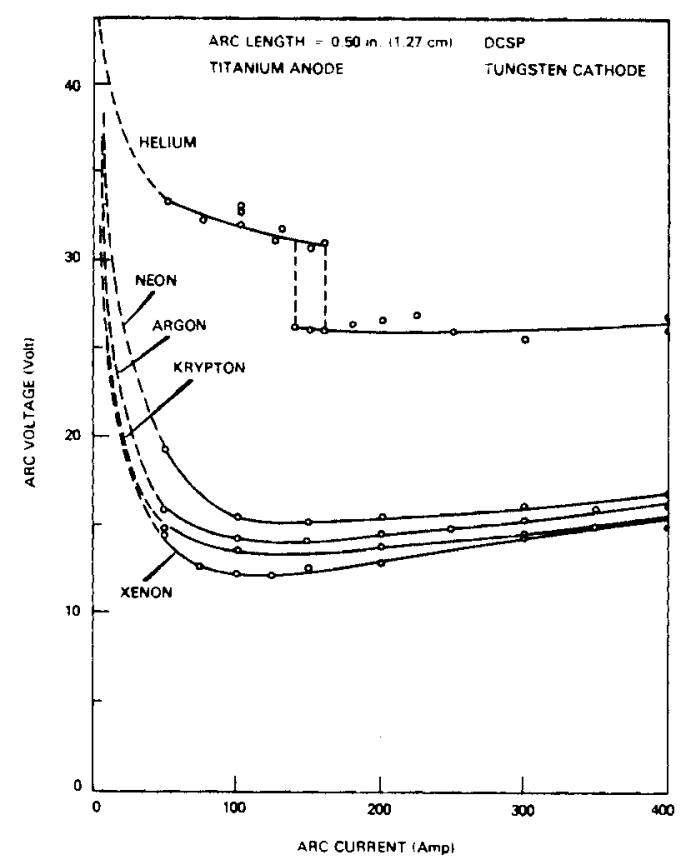

FIGURE 3. Voltwimpere Characteristics of ligh Current Arcs ${ }^{19}$ 


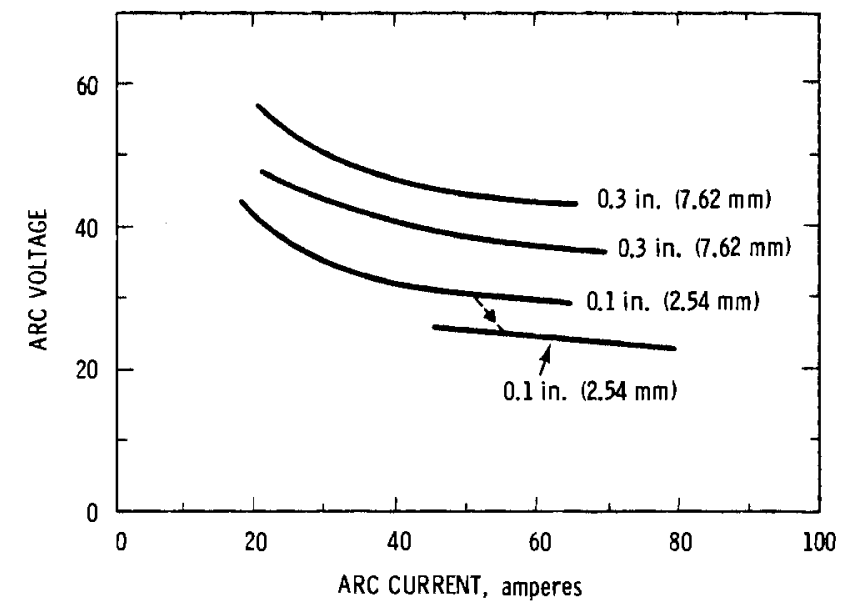

FIGURE 4A. Voltage-Current Characteristics of the Arc in Helium for Electrode Separations of 0.1 inch $(2.54 \mathrm{~mm})$ and 0.3 inch $(7.62 \mathrm{~mm})^{20}$

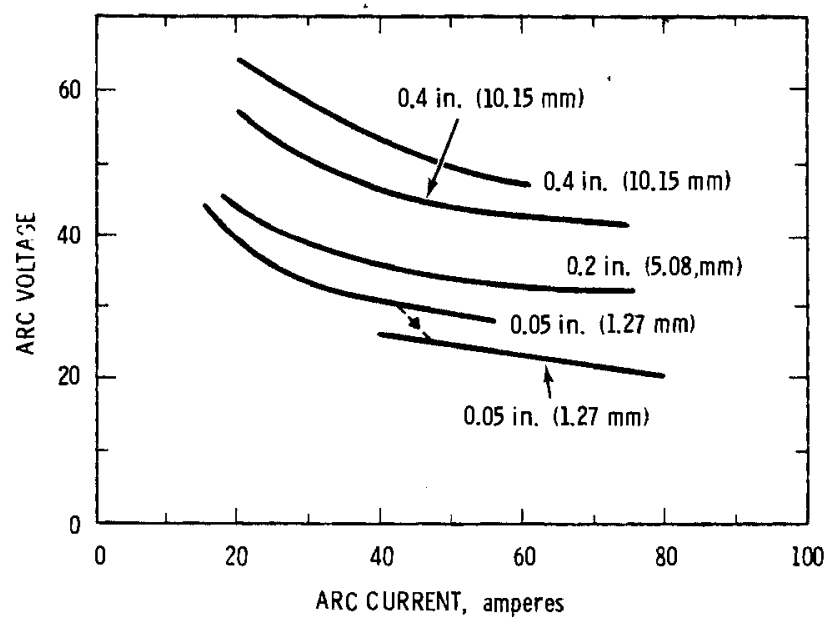

FIGURE 4B. Same as Figure 4A but for Separations of 0.05 inch $(1.27 \mathrm{~mm})$, 0.2 inch $(5.08 \mathrm{~mm})$ and 0.4 inch $(10.16 \mathrm{~mm}) 20$ 
transition is accompanied by the appearance of the blue flame of the arc and the disappearance of the reddish-pink cathode flame. For decreasing currents, the jump from the lower curve to the upper curve occurs at a lower current and the transition is more gradual. When the cathode is tungsten, the mechanism of voltage drop is associated with an appreciable increase of tungsten vapor near the cathode region. Below the transition point the plasma velocity and current density are low, while above the transition point they are both high. Transitions in practice are accompanied by changes in current that are determined by the welding power supply volt-ampere characteristics. The point at which the transition occurs is a function of the electrodes and the electrode diameters, electrode separation, and the welding current (Figures 3,4A, 4B, and 5). 21 Increasing the electrode separation with a constant-current produces a transition point in the arc voltage (Figure 5). 20 This phenomenon appears when the cathode flame makes direct contact with the anode.

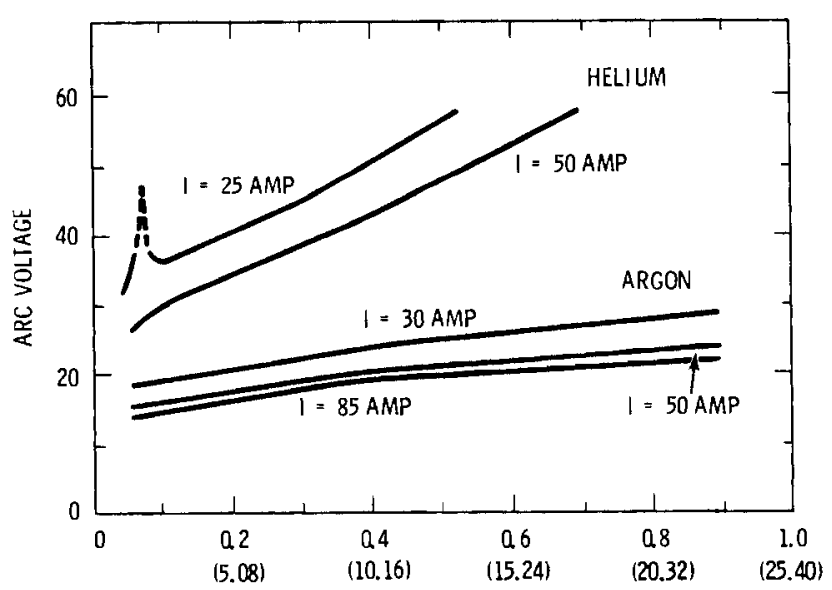

ELECTRODE SEPARATION, inch (mm)

Figure 5. Arc Voltage as a Function of the Electrode Separation 20 
The voltage gradient for mixtures of argon with increasing helium is linear up to about 75 percent helium, at which point the helium characteristic rapidly starts to predominate (Figure 6). 21 In the transition region, starting at about 75 percent helium, the arc is unstable at electrode separations where the anode flame should appear (Figures $7 A^{20}$ and $7 B^{20}$ ).*

The effect of arc length on voltage is shown in Figure 8.22 Although the voltage drop along the plasma is linear, the total arc voltage-length relationship is not linear. However, in the region of typical welding arc lengths, the usable length is so short that the arc voltage-length characteristics can usually be considered linear.

The welding arc is a point-to-plane arc whose surrounding pressure may vary from below atmospheric to above atmospheric pressure. Its many parts are confined in such a short distance as to be virtually inseparable.

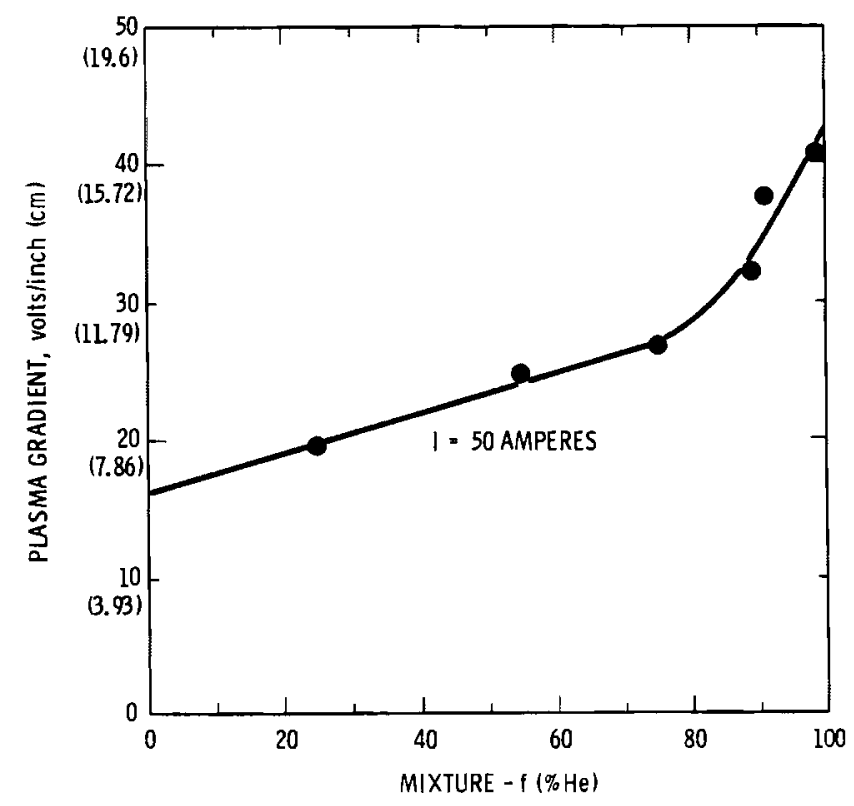

FIGURE 6. Voltage Gradient as a Function of the Mixture for $I=50$ Amperes and for Electrode Separations Between 0.1 inch $(2.54 \mathrm{~mm})$ and 0.3 inch $(7.62 \mathrm{~mm})^{20}$

*For the mechanism of the relative diameters of argon and helium arcs, see page 63 . 


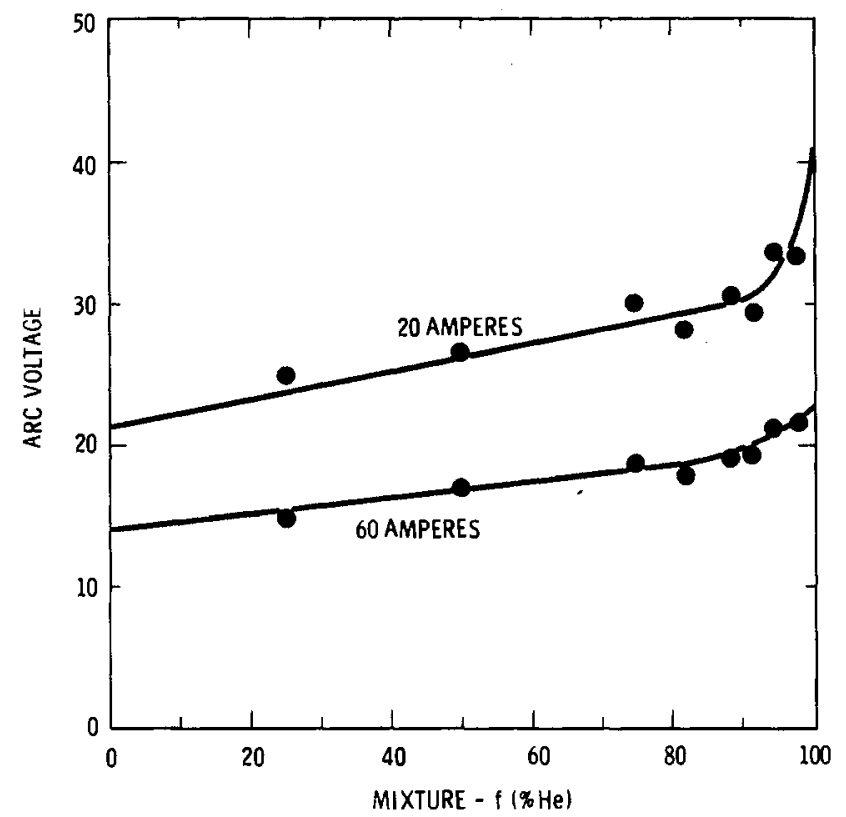

FIGURE 7A. Arc Voltage as a Function of the Mixture for $I=20$ Amperes and $I=60$ Amperes. Electrode Separation 0.05 inch $(1.27 \mathrm{~mm})^{20}$

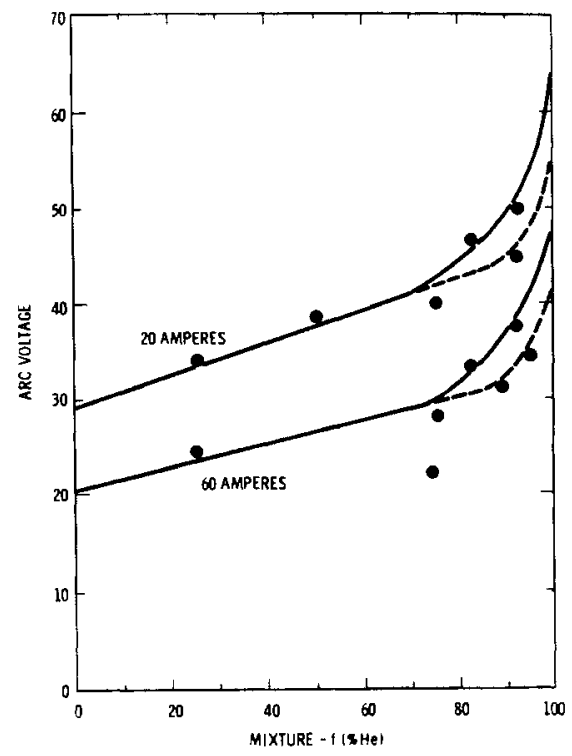

FIGURE 7B. Same as 7A But for a Separation of 0.4 inch $(10.16 \mathrm{~mm})^{20}$ 


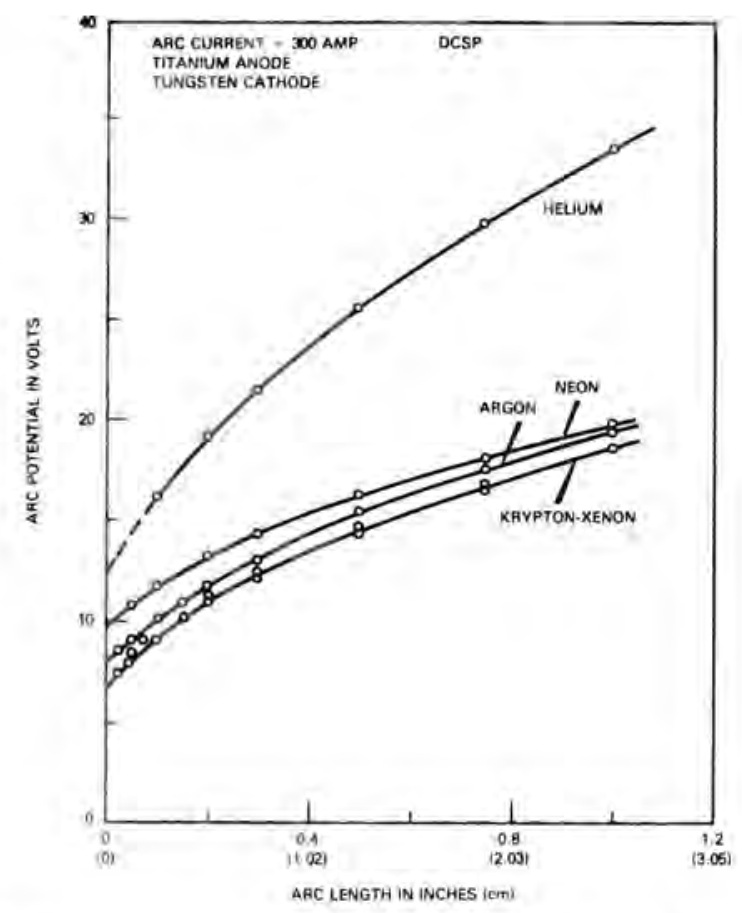

FIGURE 8. Arc Voltage-Arc Length Relationship for 3 Inert Gases and One Mixture22

Figures $9 \mathrm{~A}$ and $9 \mathrm{~B}$ show arc structure and photographs of electrode negative and electrode positive to plane arc. Their lengths have been extended in the sketches to show their components. The photographs are from a highspeed motion picture of an alternating-current 5-7 argon-helium volume ratio gas shielded tungsten arc.

The principal parts of an arc are:

- The principal cathode spot area

- The secondary cathode spots or pole spots

- The cathode fall space

- The plasma column

- Core

- Mantle

- The anode fall space

- Anode spot 

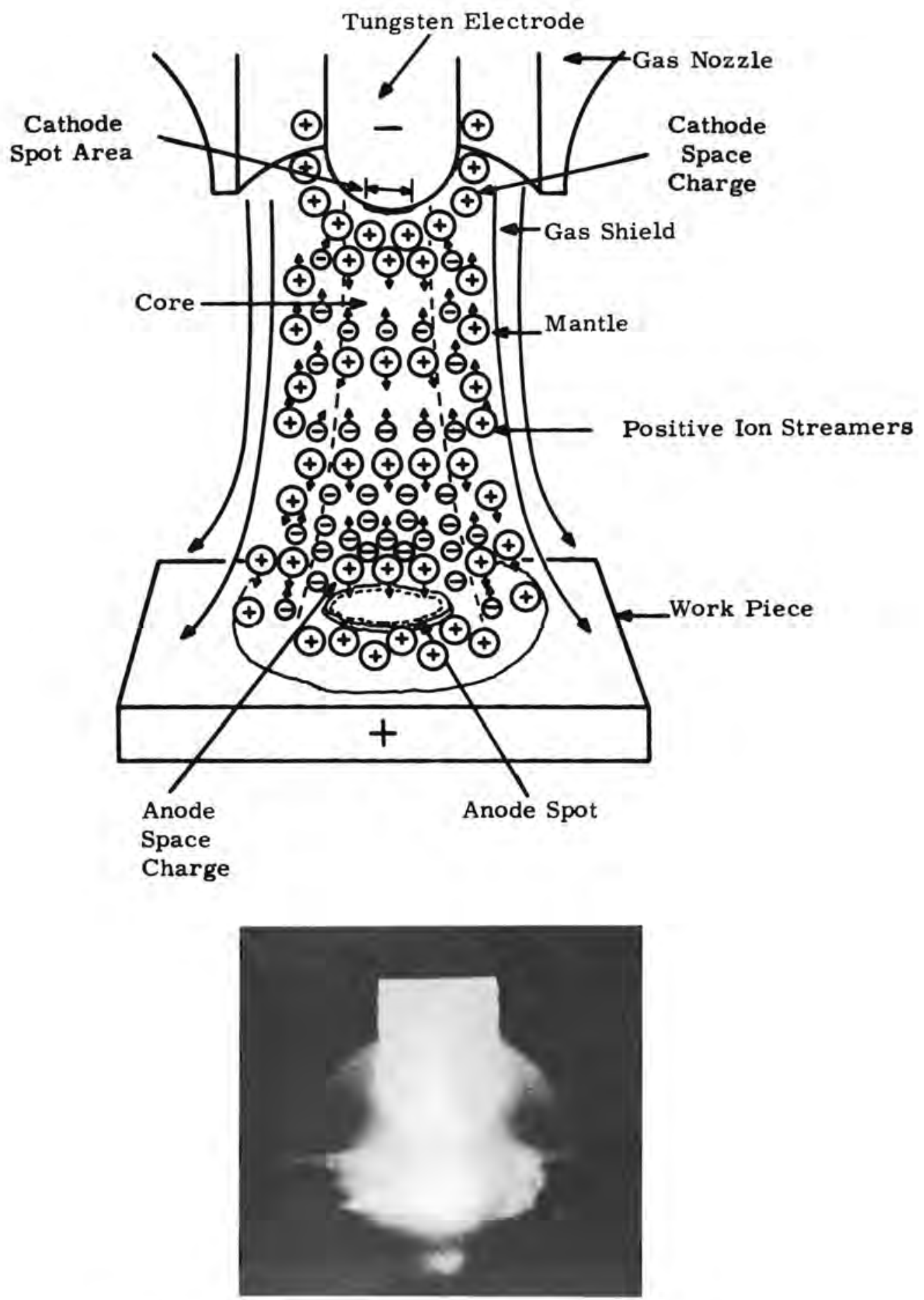

Shielding Gas 5-7 Argon-Helium Volume Ratio

FIGURE 9A. Sketch of Arcs Composition With Corresponding Photograph Electrode Negative 34 

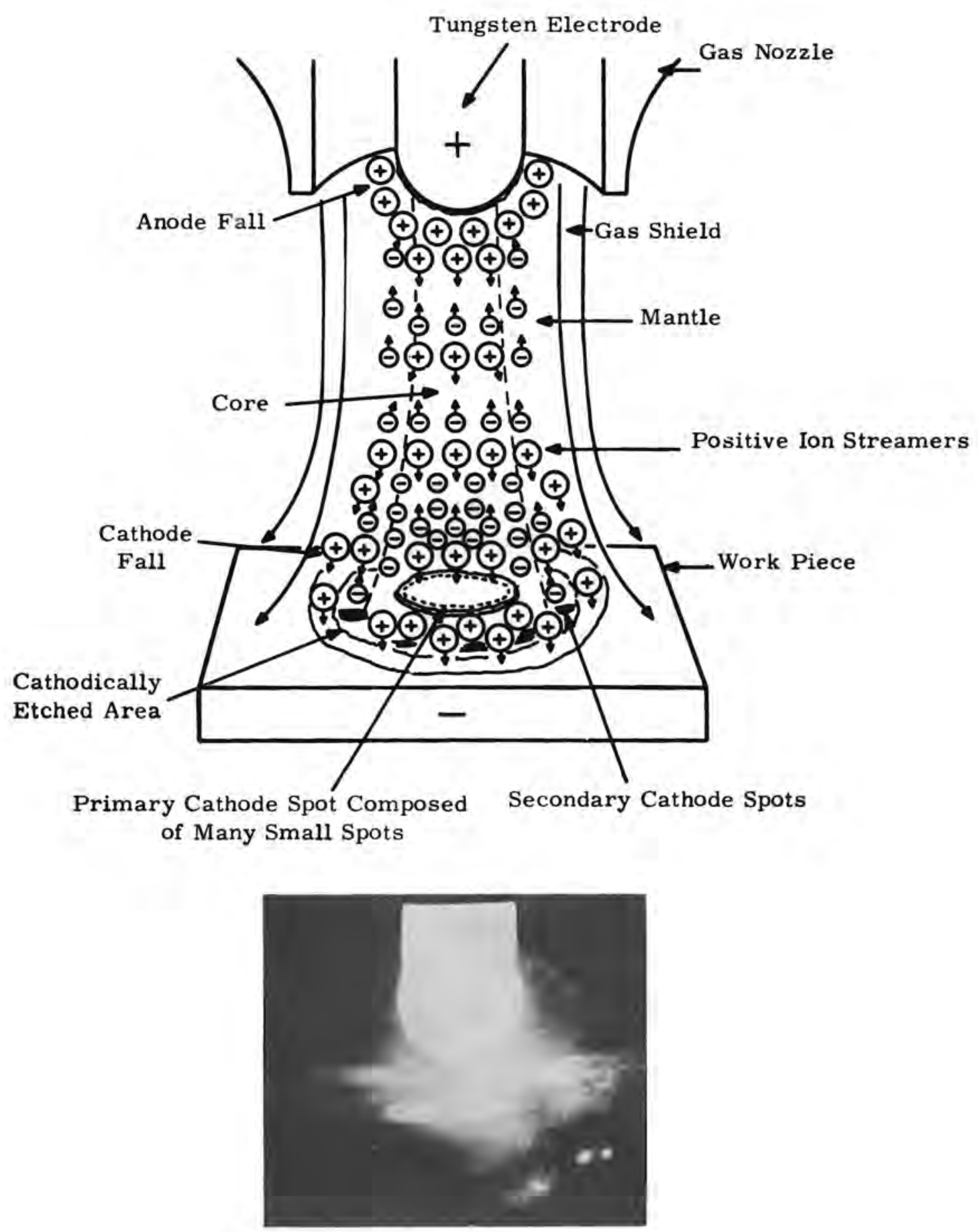

Shielding Gas 5-7 Argon-Helium Volume Ratio

FIGURE 9B. Sketch of Arcs Composition With Corresponding Photograph Electrode Positive34 
The thermionic cathode, which develops at the negative end of the arc, is a very sma11 area of extremely high current density that supplies the electrons. The electrons emitted by the cathode are accelerated through the shielding gas by the positive potential of the anode, producing the plasma by electron collision with the gas atoms. The electrons decelerated on collision with the anode produce the heat that melts the anode. As the electrons leave the cathode surface they absorb energy and exert a cooling effect on the cathode. ${ }^{23}$ At the normal operating current of thoriated tungsten electrodes the temperature of the tip of the cathode is lower than the adjacent region of the electrode due to electron evaporation. ${ }^{24}$

The positive ion sheath around the cathode provides the principal source of heat to the cathode for thermionic electron emission and melting by bombardment of the cathode. In addition, the positive ions selectively remove the oxides from the cathode before starting to remove the metal. The main and pole spots of a cathode spot resist crossing narrow, shallow scratches. ${ }^{12}$ In the cathodic etching of metals the discharge has a tendency to penetrate narrow cracks, etching both surfaces. It is not unusual when welding with inert gas shielded alternating-current or cathodic etching for the etching to fully penetrate and etch some of the back surfaces of plates $1 / 8$ to $1 / 4$ inch $(0.317$ to $0.634 \mathrm{~mm})$ thick that are either cut or fractured. Approaching the edge of plates that may be as much as $1 / 2$ inch $(12.7 \mathrm{~mm})$ thick, the cathode spots will travel over the edge and onto the back surface of the plates. 25 It is generally agreed that the mechanism of removal of all materials by cathodic etching (metals and nonmetals) is by kinetic energy exchange between the positive gas ions and the oxide and metal atoms.26,27 There is no evidence of cathodic etching when using helium as a shielding gas for welding. Its particle mass and sputtering yield are so low that the arc cathode drop voltages are unable to impart enough energy to a helium atom to dislodge very many metal atoms or oxide molecules (Figure 10).28 At the present time no explanation has been developed to explain why the positive ions selectively remove the oxide film(s) and then the metal. The action appears to take place between layers of oxide, or of oxide and metal. 

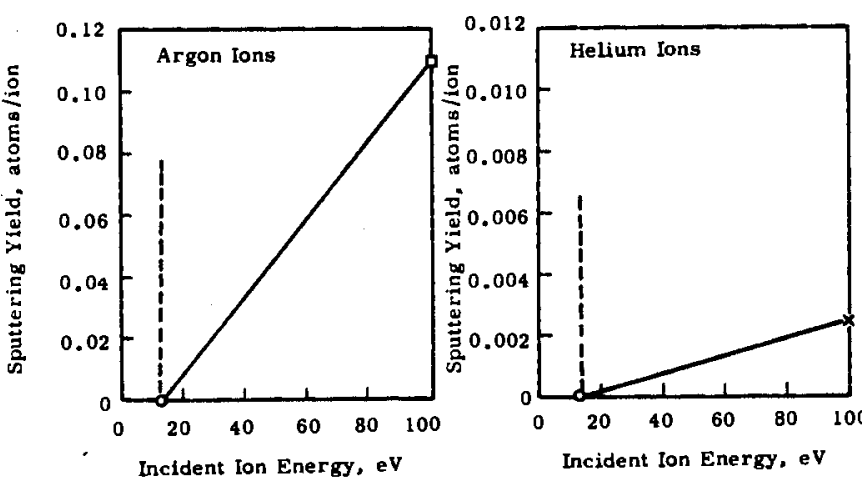

DATA SOURCES

D Laegreid and Wehner, J. Appl. Phys.. 32. 365 (1961)

Interpolation of Data from Rosenberg and Wehner,

J. Appl. Phys.. 33, 1842 (1962)

- Stuart and Wehner, J. Appl. Phys., 33. 2345 (1962).

FIGURE 10. Approximate Behavior of Sputtering Yield

With Incident Ion Energy for Argon and Helium Ions Normally Incident Upon Aluminum28

The current densities in the arc cathode spots for low and high resistivity materials may vary from $10^{4}$ to $10^{7} \mathrm{~A} / \mathrm{cm}^{2}\left(6.45 \times 10^{4}\right.$ to $6.45 \times 10^{7} \mathrm{~A} /$ in. $^{2}$ ) respectively. ${ }^{29}$ In addition, they may vary with arc current within approximately this range for arcs with single cathode spots. There is no known relation between the total arc current and the individual cathode spot currents. 30

In the case of low-resistivity electrodes, the heat required to produce the electron emission necessary to maintain the arc is generated virtually at the surface of the electrode by positive ion bombardment. The positive ions carry about 10 percent of the current. A negligible amount of heat is generated in the electrode by resistance heating, starting at the surface of the cathode spots. The combination of low resistivity and high thermal conductivity in metals makes it difficult for the arc to raise the temperature of the cathode spot(s) high enough to supply the required thermal electrons. In some cases they may be partially supplied by field emission. 31 
In refractory metals, a considerable amount of the heat necessary to produce the required thermal electrons is produced by positive ion bombardment of the cathode. 32 In addition, an appreciable amount is produced by resistance heating in the metal starting at the surface of the cathode spots. For current densities of $10^{7} \mathrm{~A} / \mathrm{cm}^{2}\left(6.45 \times 10^{7} \mathrm{~A} / \mathrm{in} .^{2}\right)$, the heats supplied by positive ion bombardment and resistance heating are approximately equal. 33 This value was determined with currents not exceeding 10 amperes. Above 10 amperes there is more than one cathode spot for most electrode materials. As the current increases the original cathode spot is surrounded by an increasing number of small pole spots. At least some of these are. transient spots that wander over the surface of the cathode at very high speeds. Their speed and random movement indicate that they may cross the central stable cathode-spot area. They are continually forming, etching the cathode, and disappearing. With higher values of current, the central cathode spot area is a multiplicity of spots. ${ }^{34}$ From the transient cathode spots observed in high speed motion pictures, their maximum life span is not more than a few milliseconds. The shortest spot life is probably a microsecond or less, just long enough to leave a minute etched spot on the cathode surface. In Figure 11 are shown cathodic etchings with grains and pits starting to appear as heavily etched areas. 25

The cathode fall space of the arc discharge is produced by the positiveion space charge which the cathode fall voltage develops at the negative end of the arc and is the order of the least ionization potential of the gas. 35 It may actually be less than the ionization potential because of ionization produced by multiple electron bombardment of single atoms.

The plasma column is the region between the anode and cathode. The core temperature of the column is determined by the current magnitude. The core temperature may vary from approximately $4000^{\circ} \mathrm{K}\left(7200^{\circ} \mathrm{F}\right)$ for $10 \mathrm{w}$ current arcs to about $16,000^{\circ} \mathrm{K}\left(27,000^{\circ} \mathrm{F}\right)$ for an arc current of 300 amperes. ${ }^{36}$ At about 30 amperes, contraction by electromagnetic forces produced by the welding current start to occur. This produces a current density in the core in the order of $3000 \mathrm{~A} / \mathrm{cm}^{2}\left(19,350 \mathrm{~A} / \mathrm{in} .{ }^{2}\right) .^{37}$ The core is considered to be the current-carrying portion of the column. There is some uncertainty as to where the core stops and the mantle begins since there is no discontinuity 

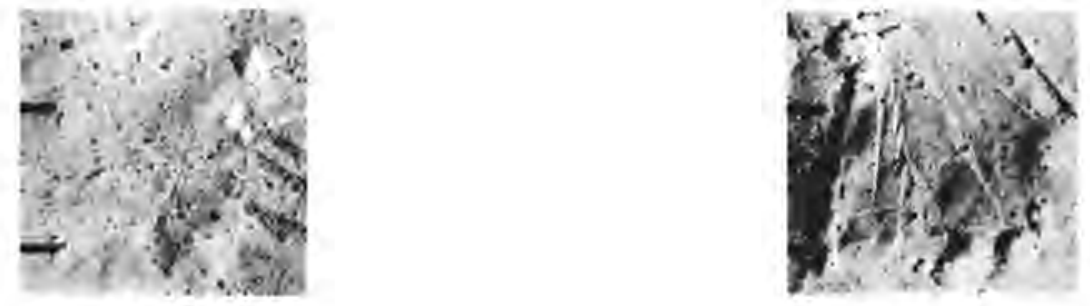

$1000 \mathrm{x}$

Incipient Etching Showing Earliest Stage as Streaks and Start of Heavy Etching as Scattered Pits

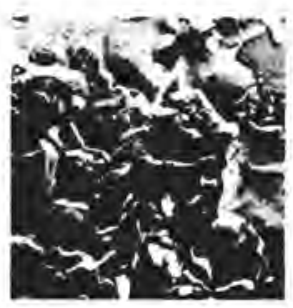

Heavily Etched

FIGURE 11. Electron Micrographs of Cathodically Etched Zircaloy-2 25

between them. The mantle is a region of chemical activity and transmits low or negligible current compared with the core. Voltage drop along the plasma column is linear as a function of arc length. 38

The anode drop is produced by the positive-ion cloud that collects immediately in front of the anode drop. It may have a voltage drop as much as the ionization potential of the gas or metal vapor. Incoming electrons produce the positive ions by collision. A higher electron-space-charge is present at the anode end of the anode-drop region. This electron space charge is due to incoming electrons collected by the anode. At high gas pressure, electrons are emitted from the anode owing to its high temperature, and contribute to the space charge until pulled back into the anode by its positive potential. ${ }^{39}$ Positive ions leaving the anode surface cool the anode. The percentage of the current carried by the positive ion(s) is a function of the element(s) in the anode and thus the net amount of heat produced in the anode. See References 48, pages 23 and 49, and page 25 . 
Electrons reaching the anode-fall space are accelerated as they pass toward the anode. On striking the anode, they give up

- The energy of evaporation they received on leaving the cathode

- The energy they received passing through the cathode-fall space

- The energy received in the plasma core and anode-fall space

thereby producing virtually all of the energy delivered to the anode spot. 40

The variation in power delivered to the anode spot by the arc is shown as a function of arc current magnitude in Figure $12^{41}$ indicating a virtually linear relationship. Though the power delivered to the anode increases with arc length, particularly with helium, experience has shown this increase is at the expense of power density.

Nothing could be found in all of the literature of the magnitude of the resistance heating in the anode spot. It appears to be rather insignificant since the anode current density is very low compared to cathode spot current density. There is no evidence to indicate that the composition of the anode

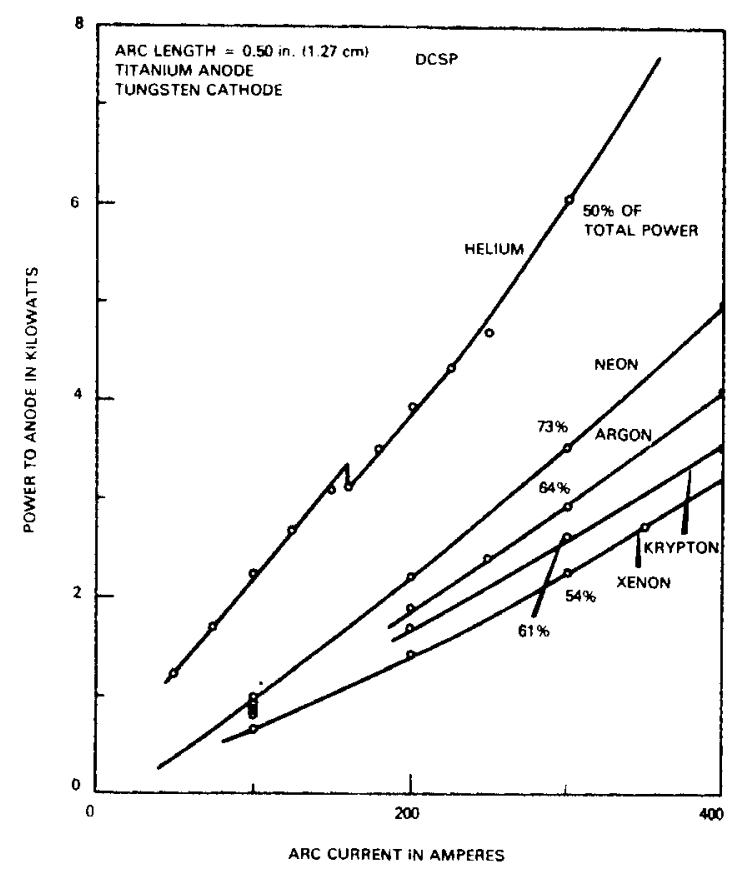

FIGURE 12. Power Delivered to the Anode-Arc Current Relationship 41 
in any way affects the volt-ampere characteristic of the arc as long as melting and evaporation of the anode do not take place.

Even though arc characteristics (volt-ampere, stability, volt-length, mode of operation, etc.) are based on mutually consistent results of several experiments, it must be emphasized that these finite characteristics are reproducible only with the finite setup. ${ }^{42}$ The finite characteristics of an arc are determined by:

- Chemical composition of both electrodes 43

- Diameter or mass of both electrodes

- Tip configuration of both electrodes

- Melting or non-melting of the anode

- Shielding gas - static or dynamic

- Cooling of the electrodes

- Shrouding of the electrodes, including a nozzle

- Alternating or direct-current

- Mode of arc operation

A small change in any one of these parameters may cause a considerable change in the arc characteristic.

1. Modes of Operation

The arc has many modes of operation and even under ideal laboratory conditions it is difficult to predict the mode of arc operation. 42 The modes are determined to a great extent by: 1) electrode composition, such as pure or thoriated tungsten, and 2) the shape of the electrode. ${ }^{44}$ It is of extreme importance to have the same mode of operation of the arc throughout the whole series of experiments. There are five known modes of operation of the electrode negative gas tungsten arc with argon shielding gas. The modes of operation are shown in Figure 13.45 A description of each mode is given in the following:

a. The cathode-spot arc has a bell-shaped envelope and an intense blue cone based on the cathode with a small dark spot (not cathode dark space) in the center of the base. This type of arc forms usually above 150 amperes. 

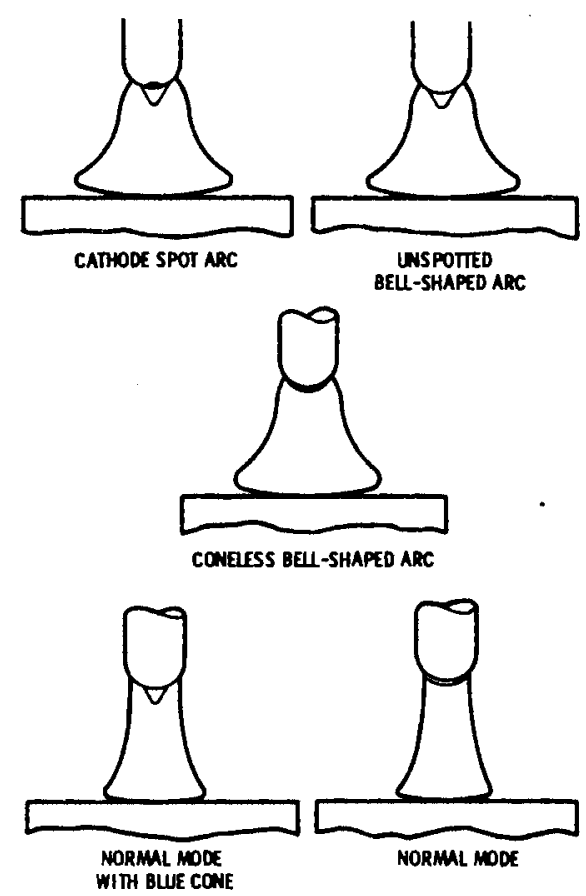

FIGURE 13. Schematic Representation of Five Types of the Argon Tungsten Arc 45

b. The unspotted bell-shaped arc is similar to the previous arc but without a dark spot. This type of arc occurred usually between 50 and 150 amperes, rarely at higher currents.

c. The coneless bel1-shaped arc has neither cathode cone nor cathode spot. It usually occurred between 100 and 150 amperes.

d. The normal mode with blue cone has an axe-shaped envelope but no cathode spot. It was formed in transition from the unspotted bel1-shaped to the normal mode and was observed usually between 30 and 90 amperes.

e. The normal mode is an axe-shaped arc without any spot or blue cone. This type exists over a wide current range.

Peak anode current densities vary from 100 to $2500 \mathrm{~A} / \mathrm{cm}^{2}$ (645 to $\left.16,125 \mathrm{~A} / \mathrm{in}^{2}{ }^{2}\right)$ and peak power transfer from 1 to $20 \mathrm{~kW} / \mathrm{cm}^{2}(6.45$ to $\left.129 \mathrm{~kW} / \mathrm{in} .^{2}\right) .46$ 
The temperatures of the surfaces of the anode and cathode must be in excess of the boiling point of most metals. Atmospheric-pressure, high frequency stabilized, low-voltage, direct-current discharges in argon gas without any evidence of a thermal cathode spot, or melting of the anode are unstable though continuously burning. The current was five amperes and the discharges were about two inches long. The colors of the light emitted at the anode and cathode ends of the discharge were determined by the anode and cathode materials. No transferring of either electrode material through the arc column was visible to the eye by a color change of the discharge. The thermal and electrical conductivities of a gas are very strongly temperature dependent (Figures $14 A^{73}$ and $14 B^{47}$ ). Thus, the discharge voltage is determined by the kinds of ions, level of ionization of the atoms, and number of each ion present in the plasma. It has been demonstrated that partial pressure of $7.5 \times 10^{-7}\left(7.5 \times 10^{-6}, \mathrm{~N} / \mathrm{cm}^{2}\right)$ atmospheres of copper vapor in a nitrogen atmosphere resulted in 97 percent of the current being carried by electrons from copper ions. ${ }^{48}$ With a current of 100 amperes in argon, the shortest two percent-thoriated tungsten arc without short circuiting on a Zircaloy-2 anode is about eight volts. The ionization potential of argon is 15.76 volts and that of zirconium 6.92 volts indicating that the current is carried mainly by electrons from the zirconium atoms. The effect on arc voltage of different metals for anodes and cathodes is shown in Figure $15.49 *$ 2. Variable Weld Penetration 50

In welding Inconel Alloy 600, vapors of $\mathrm{Ni}, \mathrm{Cr}$, and $\mathrm{Mn}$ were very clearly present in the arc; the amount was not sufficient to cause a detectable reduction in arc temperature. In addition, photographs of the arc taken with a molten anode pool revealed no noticeable difference in the shape or contour of the arc compared to those taken with a cold anode. The problem of weld penetration variability is most pronounced with automatic machine welding with the gas tungsten arc and appears to have no simple solution. In one case, the material is from two heats of metal processed

*Current, shielding gas, and arc length are not in the article and could not be obtained from author. 


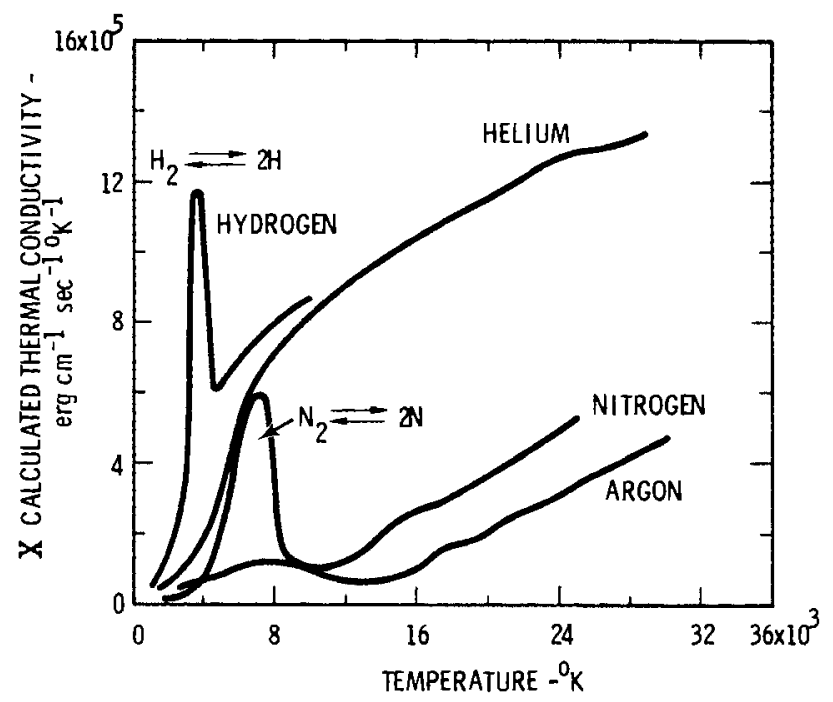

FIGURE 14A. Total Thermal Conductivity of Some Representative Gases as a Function of Temperatures 73

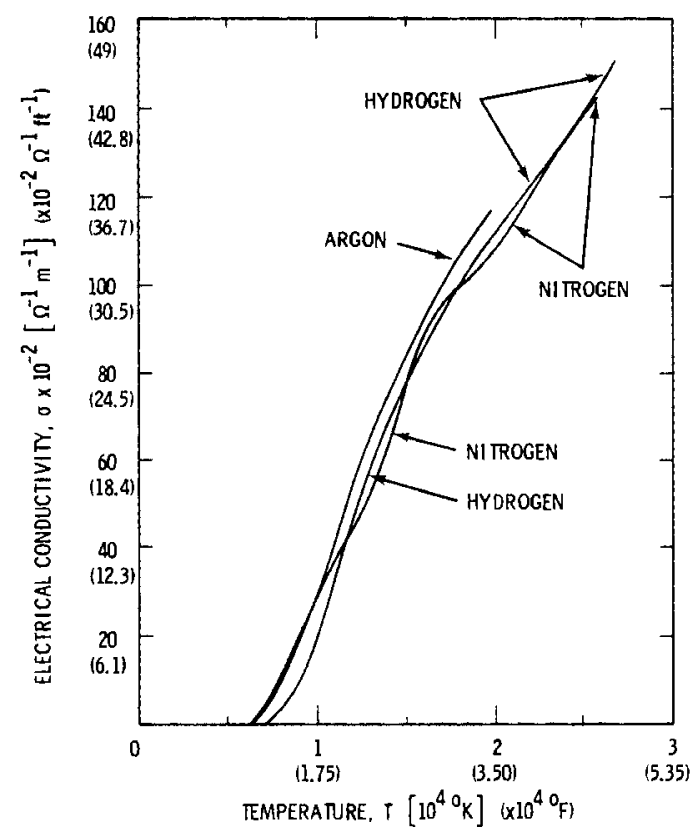

FIGURE 14B. Electrical Conductivities of Argon, Nitrogen, and Hydrogen as a Function of Temperature. 47 Similar Information for Helium Could Not be Found 


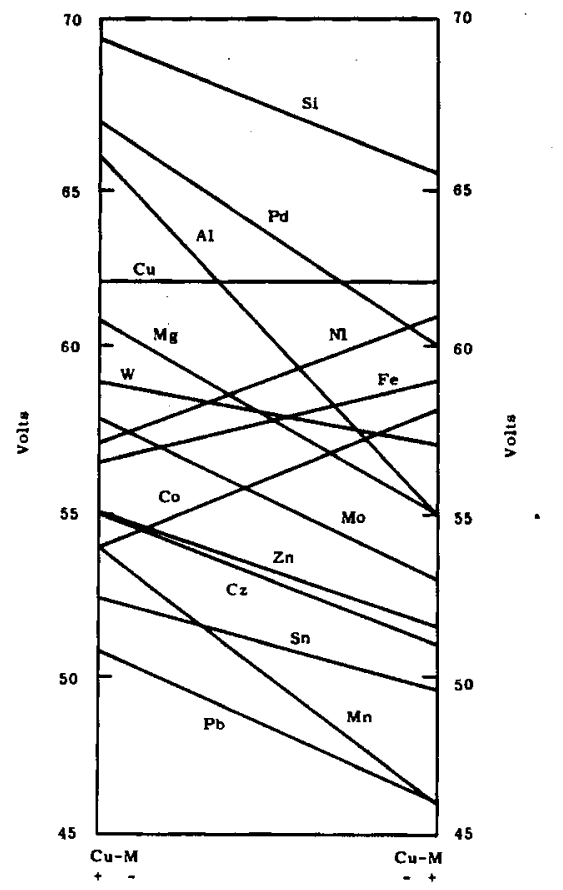

FIGURE 15. Effect on Arc Voltage of Different Metals for Anode and Cathode $49^{\star}$

exactly the same and the certifications are well within the required analysis span. In another case, one melt was inert gas arc melted and the other was vacuum arc melted resulting in a factor of ten in weld penetration and size of the molten pool. Part of the problem has been traced to infintesimal differences in the halide content of two melts, yet they were well within the specification. Others have found that the elements aluminum, calcium, magnesium, titanium, and zirconium produce high melting point slags that reduce weld penetration. One investigator noticed a very light film or dross over the surface of the molten pool that was associated with penetration reduction. There is some evidence that the presence of rare earths (cesium and lanthanum) was associated with a reduced penetration in gas tungsten arc welding of stainless steel. A group of investigators

*Current, shielding gas, and arc length are not in the article and could not be obtained from author. 
examined 20 vacuum-arc-remelted heats of 303 stainless steel and found that there was a preferred composition for silicon and manganese which promoted penetration. Another investigator found that the easily ionized impurities in the base metal which migrate into the arc produced an increase in the molten anode spot size. It was found that approximately a four-fold increase in the total impurities (oxygen and nitrogen) in the shielding gas produced approximately a 50 percent reduction in penetration. Thus, no single answer can be given for the change in weld penetration from one heat of metal to another.

\section{Arc Ignition Characteristics}

The mechanism of direct-current arc starting which takes place in less than $10^{-5} \mathrm{sec}$ appears to follow these steps:

- High frequency spark produces shielding gas ionization.

- Open circuit voltage forms a glow discharge at less than 0.1 ampere.

- Heat generated in the cathode dark space reduces its thickness, increases its area, and increases the temperature at its center; the combined effects producing thermal ionization.

- Increasing thermal ionization results in the dark space cascading its temperature and reducing its effective thickness.

- The end result is that the hot spot in the center of the cathode spot and the cathode spot transfer enough heat to the cathode (negative metal electrode) that thermionic emission takes place, and the discharge converts to a true arc with a thermionic cathode. When this takes place, the arc voltage instantly drops to a minimum value. It has been observed that for pure tungsten the arc burning voltage increases when the wandering cathode spot, such as is characteristic of the field type of emission on such metals as aluminum, changes to the fixed thermionic cathode spot. 16

- The final scene in the act is the melting, superheating, and vapor emission from the anode spot with the subsequent movement of atoms from the anode spot into the plasma. At this point, the arc stabilizes reaching its minimum voltage. There is evidence to indicate that atoms from the surface of the anode leave and enter the plasma before melting takes place. 
The cathode spot does not always initiate and stabilize on the tip of the thoriated tungsten electrode during its first few milliseconds of 1 ife as shown by cathodically etched tracks on the shank of the electrode when using argon shielding gas. The two electrodes shown in Figures $16 \mathrm{~A}$ and $16 \mathrm{~B}$ are $1 / 16$ inch $(1.59 \mathrm{~mm})$ and are two percent thoriated tungsten which has started the arc on a Zircaloy-2 anode approximately 50 times with the same current (75 amperes) and electrode spacing. The upper electrode was used in an argon atmosphere and the lower one in a helium atmosphere. There is visible cathodic etching on the argon shielded electrode. There is no visible evidence of cathodic etching on the helium shielded electrode though instability was visible at arc starting.

4. Principle of Similarity

According to the principle of similarity put forth by Steenbeck in 1932 , the potential across any region of the electric arc will adjust itself to the lowest possible level for a given current intensity. Applied to the
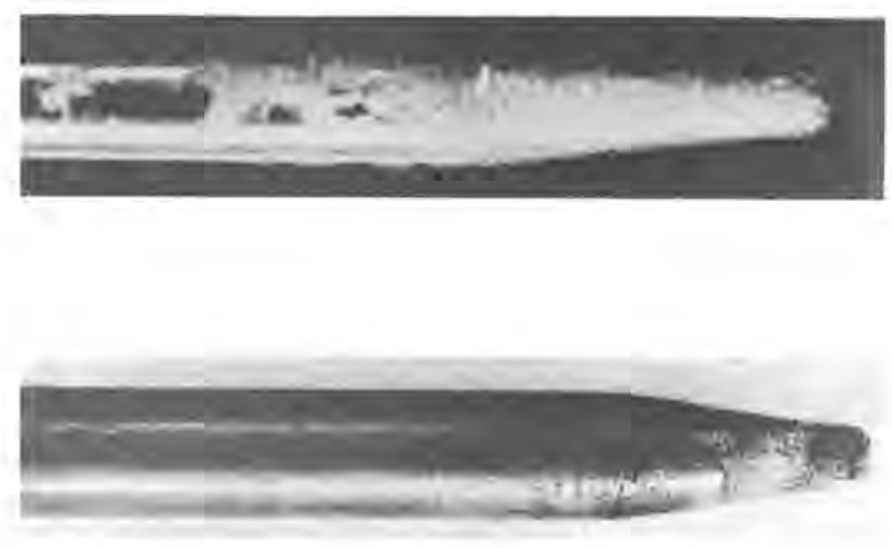

FIGURE 16A. Cathodically Etched Tracks Produced by Instability of Arc Cathode Spot During the First Few Milliseconds of Ignition in Argon (Upper)

FIGURE 16B. Helium Produces No Etching Though Instability Was Observed (Lower) 
electric arc as a whole, the minimum principle implies that any other distribution of potential between anode, cathode and arc column, or any temperature or diameter distribution other than the one chosen by the arc itself at a given current intensity will increase the total potential drop across the arc. In other words, the minimum principle states that the arc, for a given current intensity, chooses the lowest potential at conditions corresponding to the lowest possible energy production. 51,52

\section{Pole Spots}

The field intensity in front of a field-emitting cathode is of an order of magnitude of $10^{6}$ volts $/ \mathrm{cm}\left(2.54 \times 10^{6}\right.$ volts/in.), and the cathode region is about $10^{-5} \mathrm{~cm}\left(3.94 \times 10^{-6} \mathrm{in}\right.$.) 1ong. The current density of a fieldemitting cathode is not evenly distributed. It is concentrated on a very 1 imited area, the so-called cathode spot, with a diameter from $1 / 10$ to $1 / 100 \mathrm{~mm}\left(3.94\right.$ to $10^{-3}$ to $3.94 \times 10^{-4} \mathrm{in}$.) and with a current density up to $10^{7} \mathrm{~A} / \mathrm{cm}^{2}\left(6.45 \times 10^{7} \mathrm{~A} / \mathrm{in}^{2}\right)$. Near a thermally emitting cathode and near the anode a certain contraction may also be found; but the contraction is much less, $10^{-1} \mathrm{~mm}\left(3.94 \times 10^{-3} \mathrm{in}.\right)$ with a maximum current density of $10^{3} \mathrm{~A} / \mathrm{cm}^{2}\left(6.45 \times 10^{3} \mathrm{~A} / \mathrm{in.}^{2}\right) .52$

These pole spots or secondary cathode spots may be explained in general by the minimum principle. The arc tries to reduce heat losses by reducing pole areas. The minimum principle will also predict small cathode spots in field emission. As the cathode spots are reduced in size, the cathode temperature increases. This causes the thermal emission part of the total emission to increase, with the final result that the cathode drop increases. It must, however, be admitted that much about the nature of the pole spots is still obscure. In electric arc welding with field emission, the pole spots are not stationary; they wander on the cathode with a speed on the order of $100 \mathrm{~m} / \mathrm{sec}(328 \mathrm{ft} / \mathrm{sec})$, suddenly disappearing only to return again. It is believed that the arc, in accordance with the minimum principle, seeks out the most favorable locations for electron emission, i.e., locations of low wark function, but that these locations only fulfill the required conditions very temporarily. 53 


\section{Effect of Electrode Geometry}

The width, depth of penetration, and surface finish produced with the electrode negative inert-gas tungsten-arc are virtually determined by the composition, diameter, and geometry of the tip of the tungsten electrode. ${ }^{24}$ Varying the anode composition will not affect the results unless melting takes place. ${ }^{54}$ Generally speaking, the smaller the diameter and the blunter the tip of the electrode, the deeper the penetration for a finite value of current. ${ }^{45}$ As with all generalizations, there are exceptions. In welding thin plate, sheet, or root passes, the small blunt electrode does not produce the smallest diameter and the deepest penetration. ${ }^{55}$ Using a 1/8-inch $(3,18 \mathrm{~mm})$ diameter two percent thoriated tungsten electrode having a 30 degree included angle point, the smaller the diameter of the truncated point the lower the value of current required to produce full penetration, all other factors remaining the same. For the same application, the use of a broad angle point produces a much wider fusion zone to produce full penetration, the required current increasing with the angle. 55

A rough emitting surface on the electrode tip, particularly grooves that are concentric with the circumference of the electrode, produce essentially step changes in the longitudinal movement of the arc over the emitting surface as the current is either increased or decreased. 56 This is produced by the cathode end of the arc moving very freely over a smooth surface but virtually refusing to pass over a shallow scratch. ${ }^{12}$ During precision welding this produces variable weld width, penetration, and surface roughness.

The electrode diameter and tip contour used in mechanized directcurrent electrode negative gas tungsten-arc welding influences greatly the depth of penetration and width of the fusion zone (Figures $17^{57}$ and $18^{57}$ ). With a finite angle of the electrode tip, varying the current over the upper 75 percent of the current range of the electrode while holding the electrode to work distance and travel speed constant, the depth to width ratio of the fusion zone will remain virtually constant. In welding with pulsed current, maximum depth to width ratios of the order of two to one may be obtained by the smallest diameter two percent thoriated tungsten electrode whose tip 


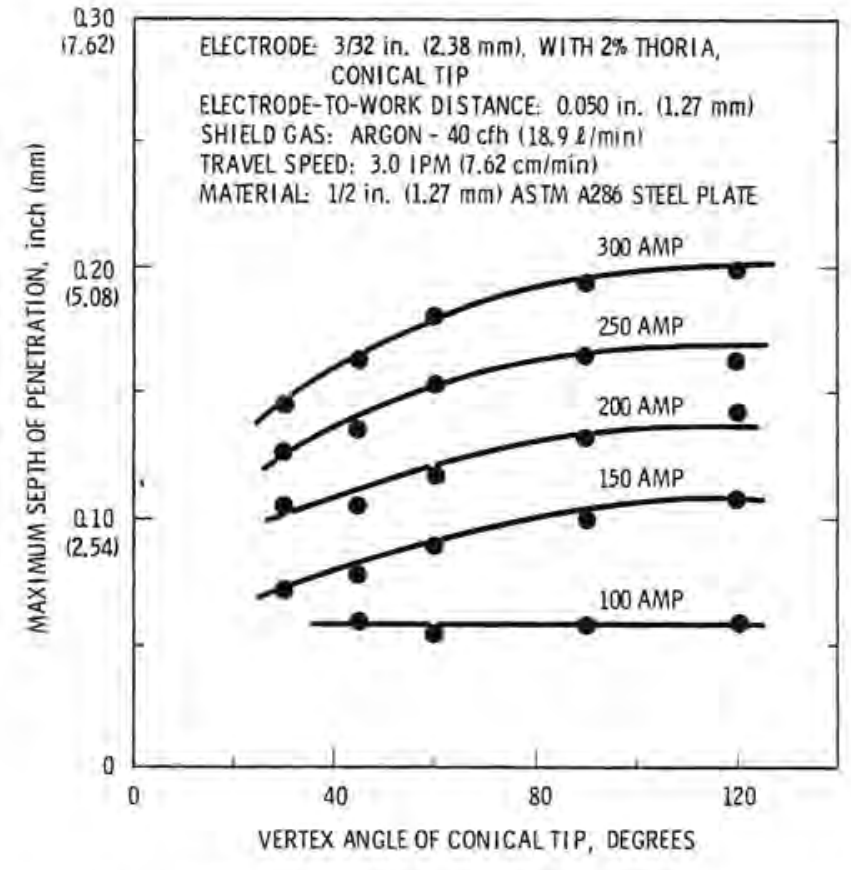

FIGURE 17. Effect of Vertex Angle of Conical Tip on Depth of Penetration 57

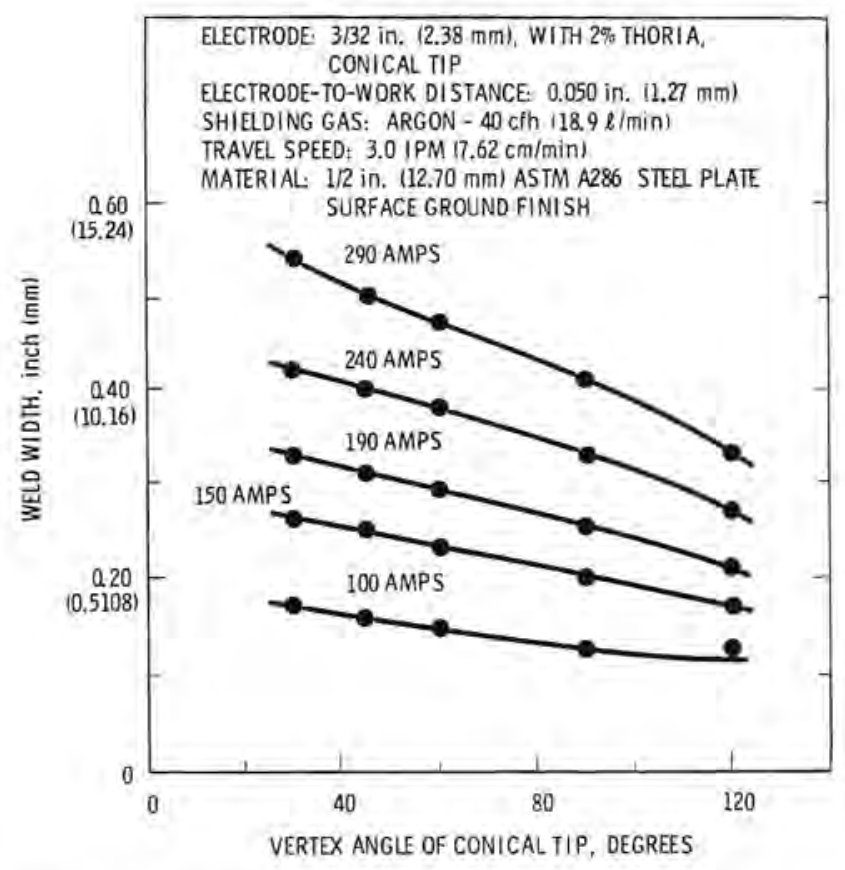

FIGURE 18. Effect of Vertex Angle of Conical Tip on Weld Width ${ }^{57}$ 
will not melt or whose end will not fall off due to heating within the nozzle. The point must have a 90 degree or greater included angle. Penetrations through stainless steel on the order of $1 / 4$ inch $(6.36 \mathrm{~mm})$ thick have been accomplished using small diameter electrodes, high current, and short duration single pulses of current. When this phenomenon is applied to metal joining it is called PIGME ${ }^{58}$ welding (Programmed Inert Gas Multiple Electrode).

\section{Mixed and Contaminating Gases}

It is virtually impossible to maintain an absolutely pure gas or an absolute mixture of gases. However, if the contents of a mixed shielding gas varies from its composition, particularly mixtures of helium and argon, the depth-to-width ratio and depth of penetration will change appreciably. Suppliers* of mixed gases recommend that before use the gases should be agitated mechanically or thermally to eliminate stratification. Contaminants such as water vapor and nitrogen exert their influence by changing the electrode negative tip configuration and arc voltage. In Figure 19 is shown a $1 / 4$-inch $(6.36 \mathrm{~mm})$, two percent thoriated tungsten electrode that was

\footnotetext{
FIGURE 19. Erosion of Tungsten Electrode by Water Vapor In the Shielding Gas** 1/4" $(6.3 \mathrm{~mm})$

$2 \%$ Thoriated Tungsten
}

\footnotetext{
*Matheson, Lyndhurst, N.J. 07071

**Courtesy of United Nuclear, New Haven, Connecticut
} 
operated in a helium atmosphere containing a trace of moisture. The loss of tungsten was produced by the evaporation of the tungsten oxide formed by the reaction between the tungsten and water vapor.

\section{Magnetic Arc Blow*}

Arc blow is the deflection of the flexible welding arc column by magnetic forces produced by the welding current and possibly by extraneous magnetic fields. The effect of the magnetic forces is analyzed most convenientTy by means of diagrams of the magnetic flux. In Figure $20 \mathrm{~A}^{59}$ is shown the magnetic field produced by the current flowing through the conductor, and in Figure $20 \mathrm{~B}^{59}$ the fields produced by:

- Current flowing in the conductor

- External field

- Resultant field

The lines of force on one side of the conductor add to the external 1 ines of force; on the other side of the conductor they subtract from the external lines of force. The net force produced by the difference in the number of lines of force on the two sides of the conductor produces a force at right angles to the conductor; the force is toward the less dense side (Figure 20B). 59 The direction in which the current flows, as long as the magnitudes are equal, has no influence on the magnitude of the arc deflection produced by the reaction between the flux produced around the arc by the welding current and the flux produced by the welding current in the magnetic material. When the current return connection is near the arc, the magnetic field is as shown in Figure $21 \mathrm{~A},{ }^{59}$ blowing the arc column and end in contact with the plate away from directly below the electrode. This can be eliminated by providing a second return lead (Figure $21 \mathrm{~B}$ ) ${ }^{59}$ in such a way that the magnetic field around the arc is symmetrical. The 1ocally magnetized areas within the weldment must be removed before welding to produce a uniform magnetic field by the welding current.

*For a more informative and detailed discussion of magnetic control of welding arcs see supplemental references. 


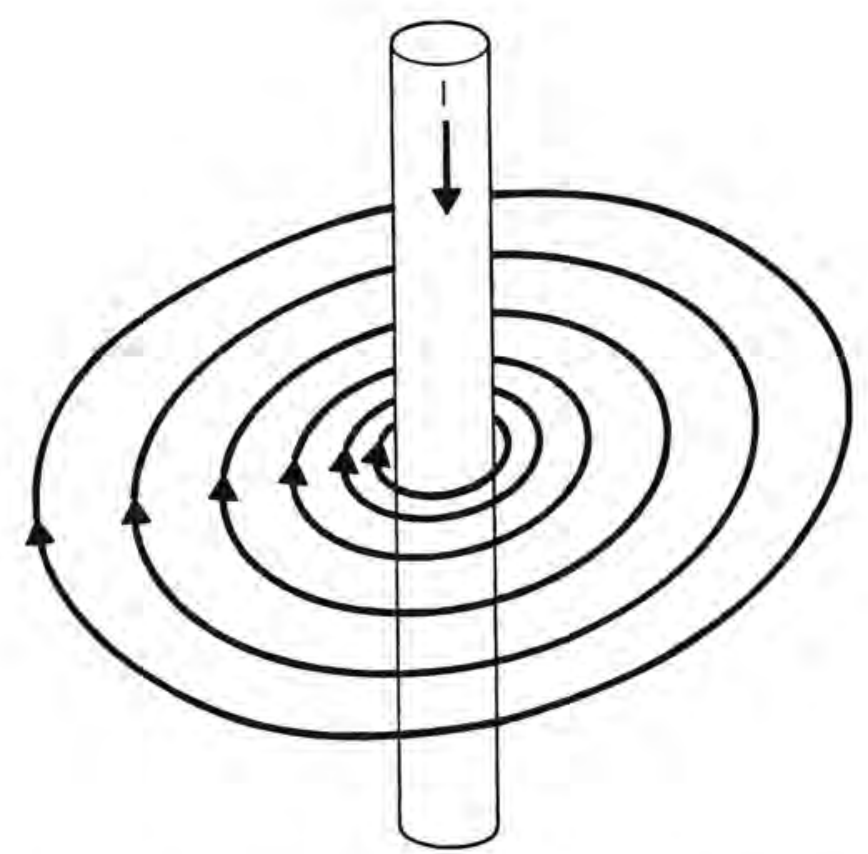

FIGURE 20A. Magnetic Field Around a Conductor 59

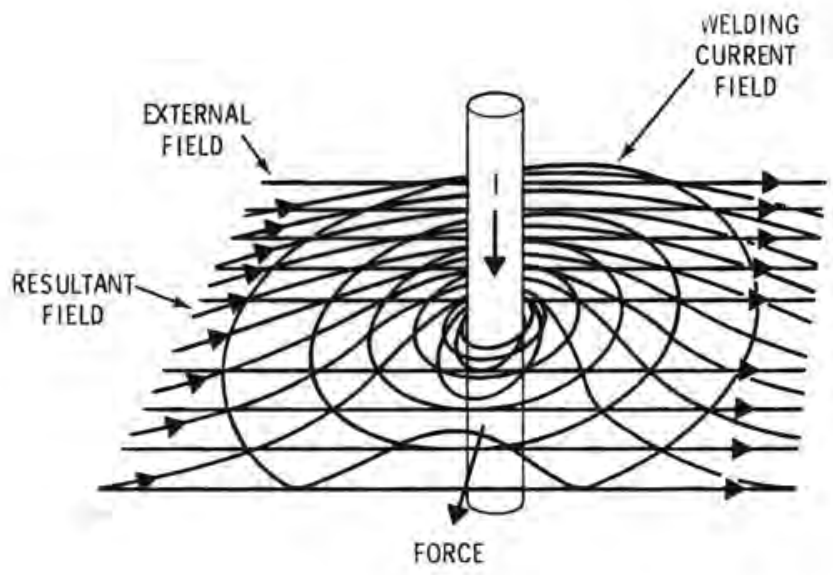

FIGURE 20B. Magnetic Field of a Conductor in Interaction With an Outside Magnetic Field 59 


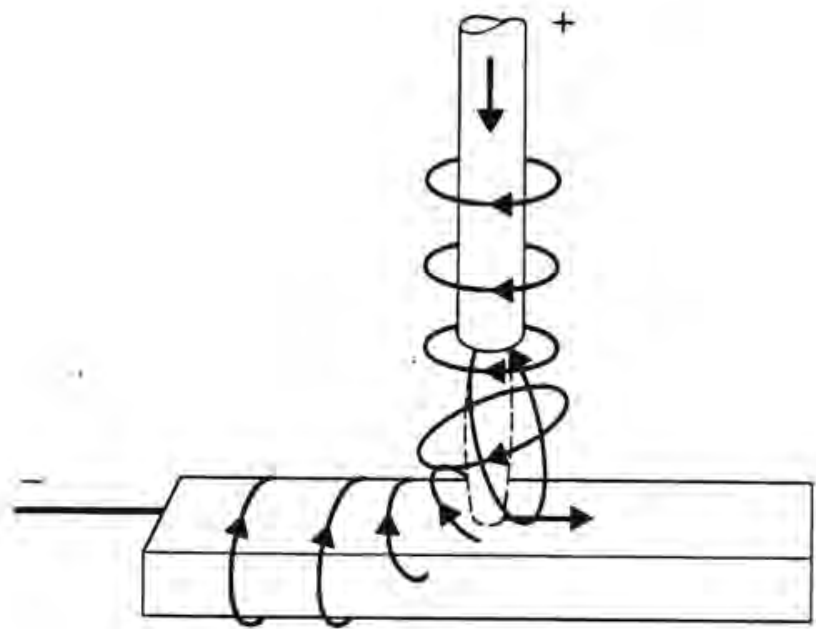

FIGURE 21A. Magnetic Field of an Arc in the Proximity of Its Return Connection 59

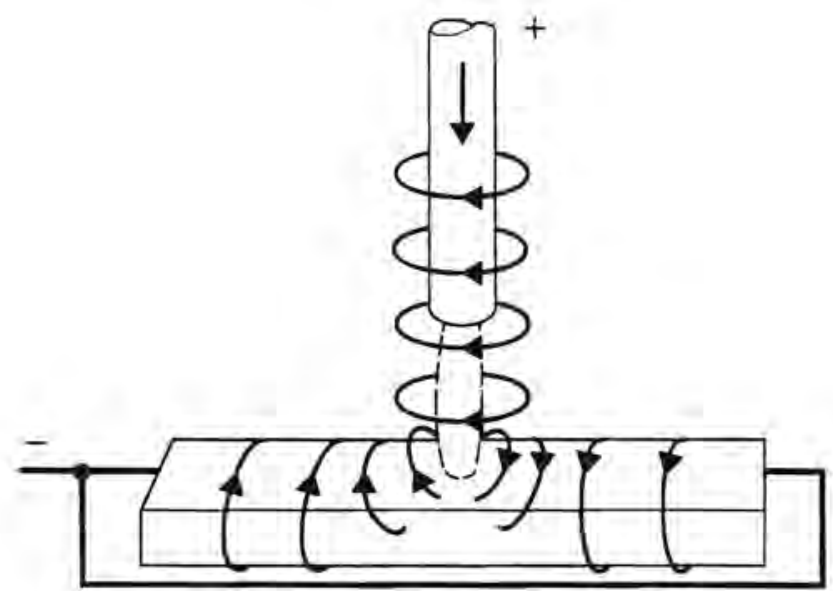

FIGURE 21B. Magnetic Field in the Case of the Balanced Return Connections 59 
When welding with alternating-current, eddy-currents are induced in the work. These currents produce a field of their own which tends to neutralize the field produced by the arc current. Welding with alternating-current reduces the arc blow significantly below that produced when welding with direct-current. When welding magnetic materials, the eddy-currents may produce a resultant force on the arc. This occurs when the eddy-currents are not symmetrically located with respect to the arc. Material in the weld puddle and extending out some distance around the puddle in an asymmetric pattern (Figure 22) $)^{59}$ is raised above the Curie point, about $730^{\circ} \mathrm{C}(1346 \mathrm{~F})$ and is non-magnetic while the material outside this area retains its magnetic properties. The field produced by the welding current in the magnetic material is moved away from the electrode and has an asymmetric pattern. The eddy-currents produce a resultant force in the direction of welding (Figure 22). $\star^{59}$

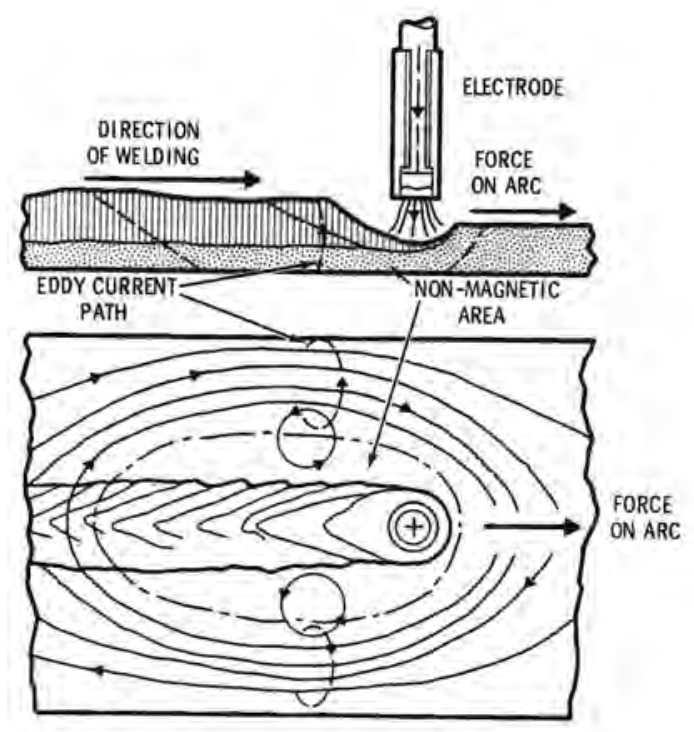

FIGURE 22. Assymetric Location of Eddy Currents Caused by Heated Non-Magnetic Area ${ }^{59}$

*For electromagnetic control and positioning of the welding arc see supplementary references $22,23,24$, and 25 . 


\section{Phenomena of Travel Speed and Arc Stability}

In a point to plate arc with the electrode-negative and the plate a long strip of carbon stee1, as the arc starts and melting of the strip takes place, superheating of the anode spot in the resulting puddle takes place. Metal vapors from the anode spot enter the arc column. The combination of these factors lowers the arc voltage and anchors the arc to the anode spot. Any change of horizontal or vertical spacings between the anode and cathode will produce a change in the voltage of the arc.*

As the anode strip accelerates from the stationary starting position, the width and depth of the fusion zone decreases and the temperature of the superheated molten anode spot decreases reducing the number of ions entering the plasma from the anode. The molten anode puddle starts lagging behind the cathode (Figure $23(\mathrm{a})$ ). ${ }^{60}$ The sum of these changes produces an increase in arc voltage.

With a further increase in the strip speed, a point is reached at which the molten anode puddle ceases to move (Figure $23(b)) .60$ As the arc lengthens, the arc column between the anode and cathode bends toward parallelism with the moving anode surface. The closest point of the plasma to the anode, in relation to the cathode, is virtually below the end of the cathode. When the voltage between the plasma on the end of the cathode and the anode strip becomes sufficiently high, the shortest distance between the plasma and the anode surface forms a preferred position for the formation of a new arc (Figure 23(c)). 60 The new arc starts burning when its ignition voltage is attained or exceeded slightly, dropping the voltage on the arc at the old location, causing it to extinguish and transferring a11 of the current to the new arc. High speed motion pictures at the rate of 3000 frames per second show that when the arc jumps to a new location a new anode is formed simultaneously. Coincident with the anode spot

* For this discussion, the cathode is considered stationary and the strip travels. Physically, the converse would produce equal results if windage from the electrode speed would not interrupt the shielding gas surrounding the electrode. 


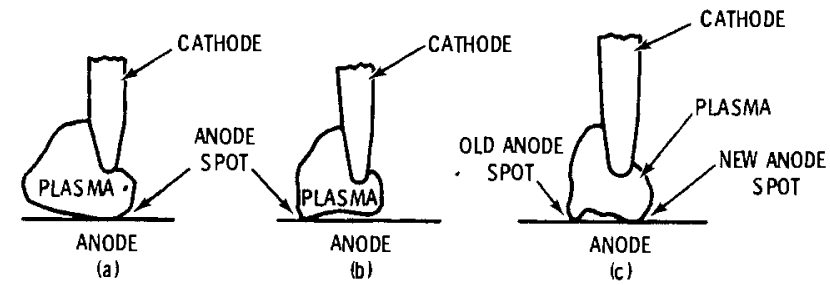

FIGURE 23(a), (b), \& (c). Arc Formation and Extension Three Drawings of Motion Picture Frames Blue Filter Carbon Cathode, 52 Amperes Anode Speed, $25 \mathrm{ft} .,(7.62 \mathrm{~m})$ Per Minute 60

formation is the appearance of a high velocity jet, consisting of vapor and incandescent particles, projected outward from the spot, and perpendicular to the anode surface (Figure 24). ${ }^{60}$ 0scillography shows there is a slight decrease in current at the point the new arc initiates but no discontinuity in the current, producing a smooth transfer of current from the old arc to the new one. Performance of this process is related to the material of the cathode, which is the source of electrons that carry the major portion of the arc current, and is also influenced by the arc atmosphere and the composition of the anode. As the strip continues to move at constant speed and current, uniformly spaced, fused spots of uniform diameter are produced with centers raised above the anode surface. Increasing the anode travel speed beyond this point increases the spacing of the anode spots a rather small amount (Figure 25). ${ }^{60}$ Increasing the current at constant anode travel speed decreases the spot spacing (Figure 26), ${ }^{60}$ eventually reaching the point that the anode spot starts to travel, forming a continuous fusion 


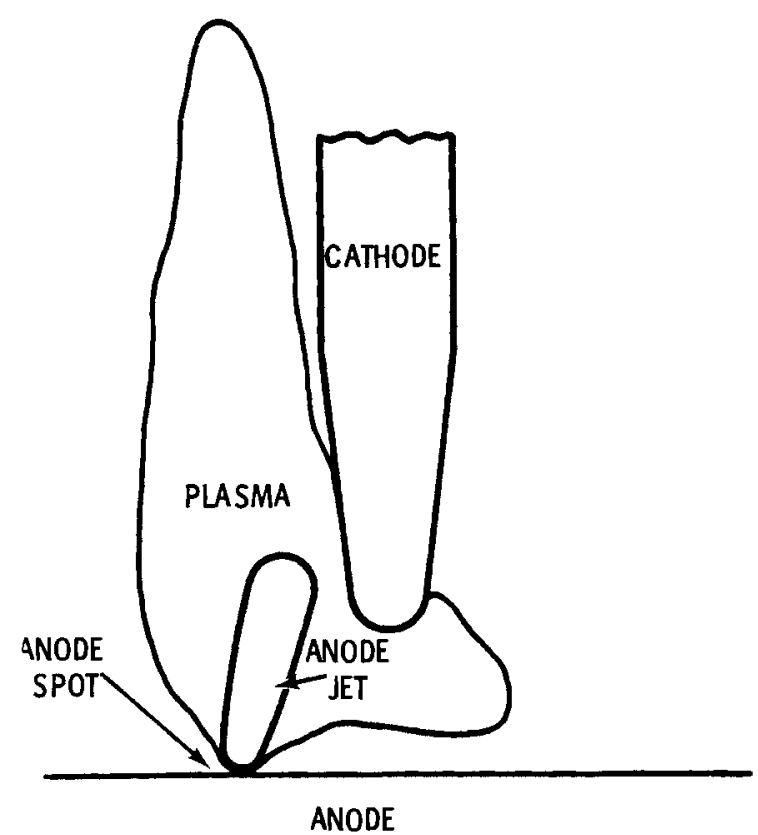

FIGURE 24. Anode Jet. Red Filter. Carbon Cathode, 52 Ampere. Anode Tape Speed 25 Feet

(7.62m) Per Minute60

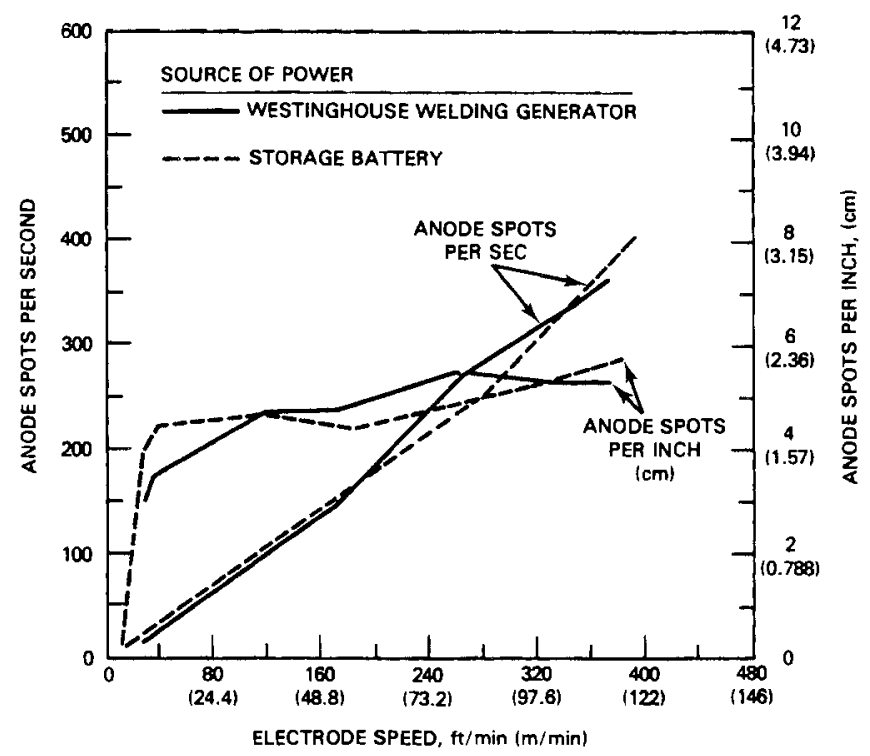

FIGURE 25. Variation of Anode Spot Characteristics With Electrode Speed. 60 


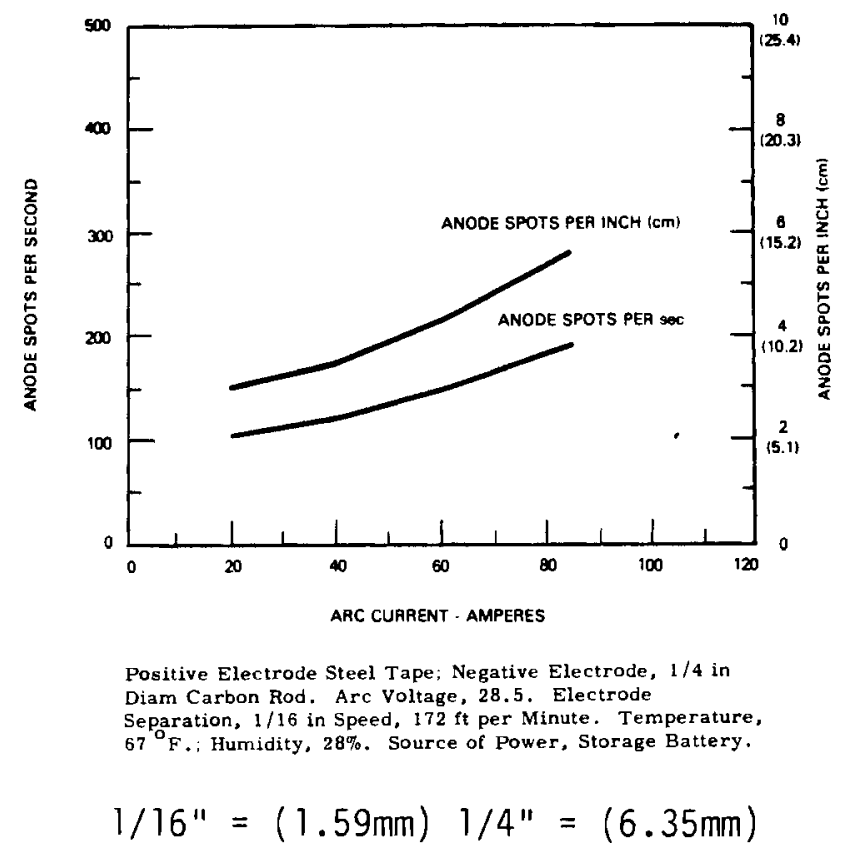

FIGURE 26. Variation of Anode Spot Characteristics With Current 60

path.* Conversely, decreasing the current increases the anode spot spacing until the arc voltage can no longer maintain the arc--the arc becomes unstable and extinguishes.

Increasing the arc length at constant current and travel speed increases the arc burning voltage, and thus the spacing between the spots, as the plasma must be lengthened to produce the ignition and burning voltages required for increased arc length (Figure 27).60

In Figure $28^{61}$ is shown the variation of the area of the individual anode spots produced with variation of current at constant travel speed. The linear relationship with current indicates that over the speed and current ranges covered, the heat that is causing the steel surface to melt is produced by a constant voltage arc.

*Continuously molten puddles have been maintained at 5000 I.P.M. $(127,000 \mathrm{~mm} / \mathrm{sec})$. 


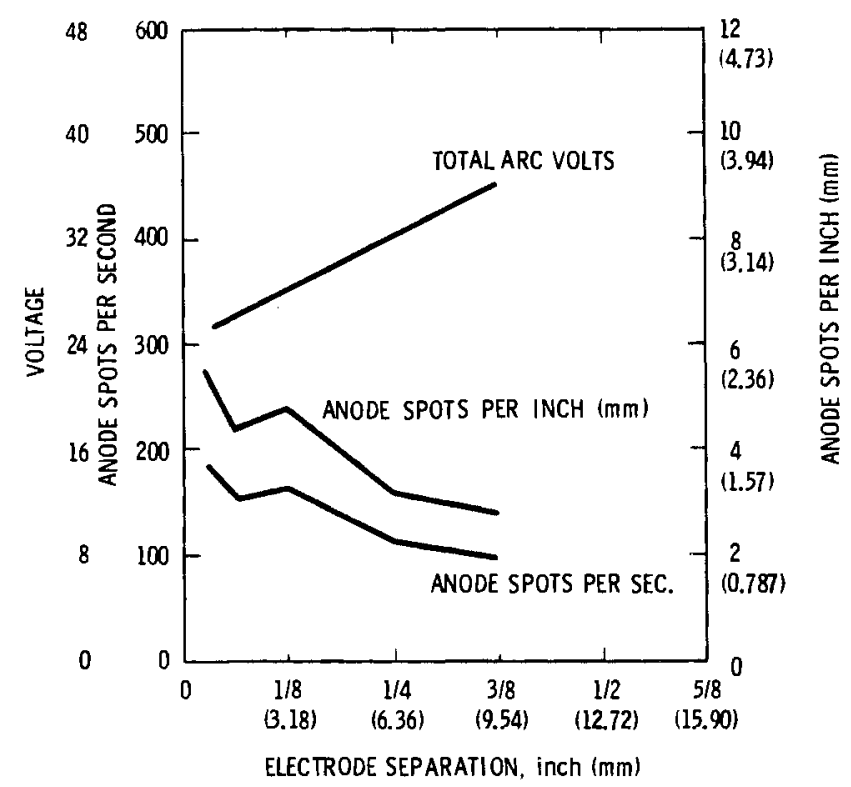

FIGURE 27. Variation of Arc Characteristics With Electrode Separation 60

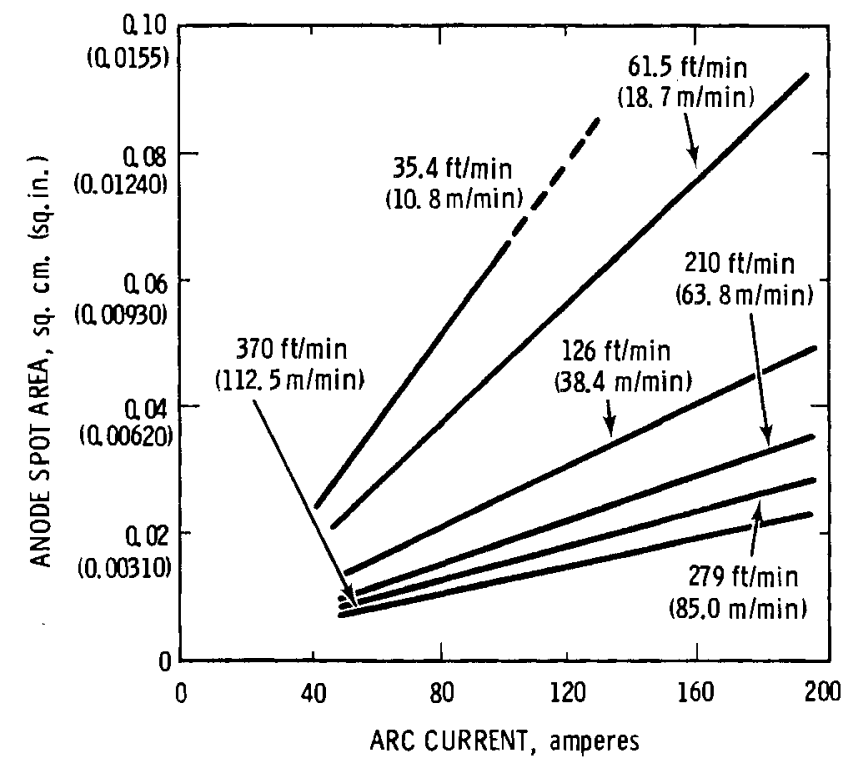

FIGURE 28. Variation of Area of Individual Anode Spots With Total Arc Current61 
Shown in Figure 2961 is the rate of anode melting as a function of strip speed. It is seen that the lateral rate of melting increases in an approximately linear manner as the relative electrode speed is increased. This indicates that the heat which produces melting of the anode surface is most efficiently utilized at high rates of relative electrode speeds.

The variation of the area of individual anode spots with electrode speed is shown in Figure 30.61 Using the data from these curves and from Figure 28,61 the relationship between the area of the individual anode spots and the time in milliseconds that the arc is burning in each spot was determined (Figure 31).61 The intercepts of these curves with the horizontar time axis should give a measure of the time required to begin melting the steel surface in an anode spot.

The variation in the apparent current density with electrode speed is shown in Figure 32.61 This should indicate the order of magnitude of current densities involved to a closer degree than can be obtained using a stationary electrode system.

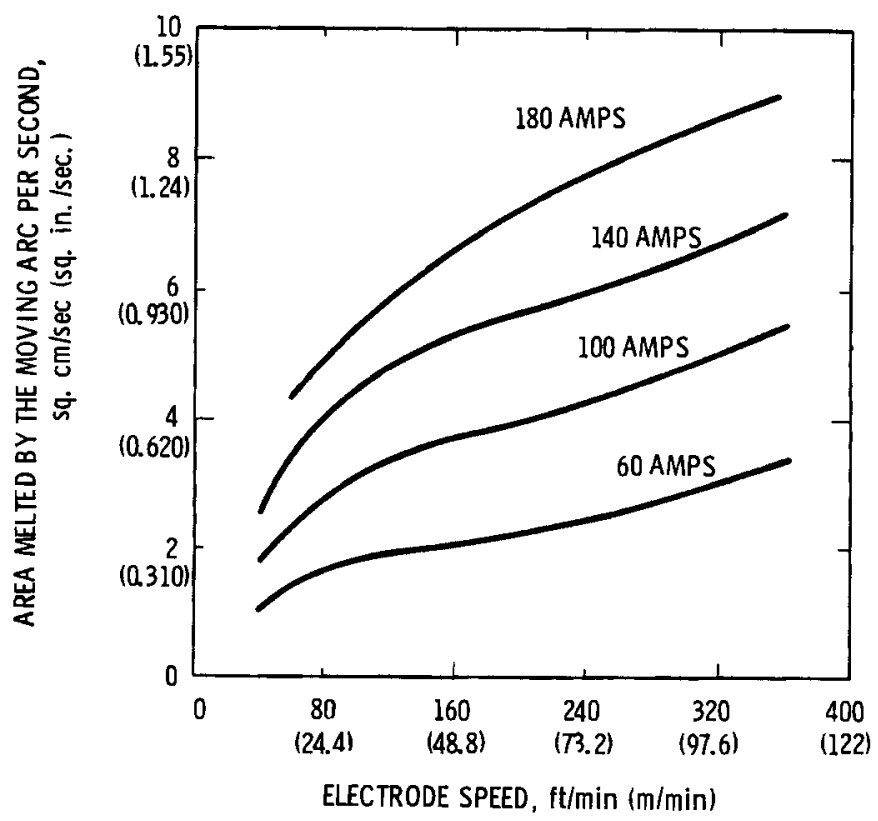

FIGURE 29. Variation of Total Lateral Area Melted Per Second With Speed of the Moving Tape 61 


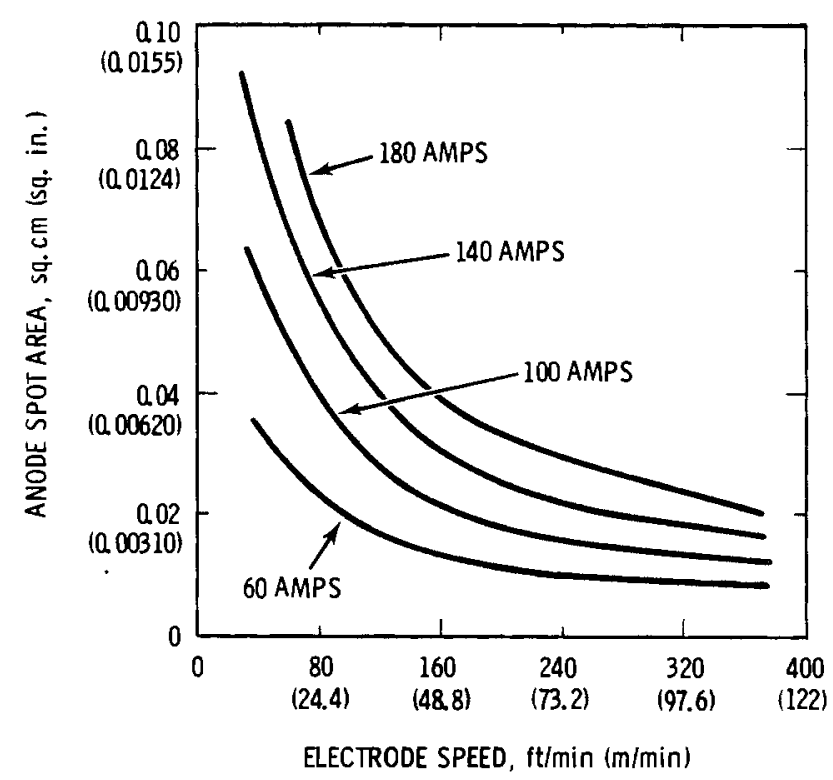

FIGURE 30. Variation of the Area of the Individual Anode Spots With Speed of the Moving Tape ${ }^{61}$

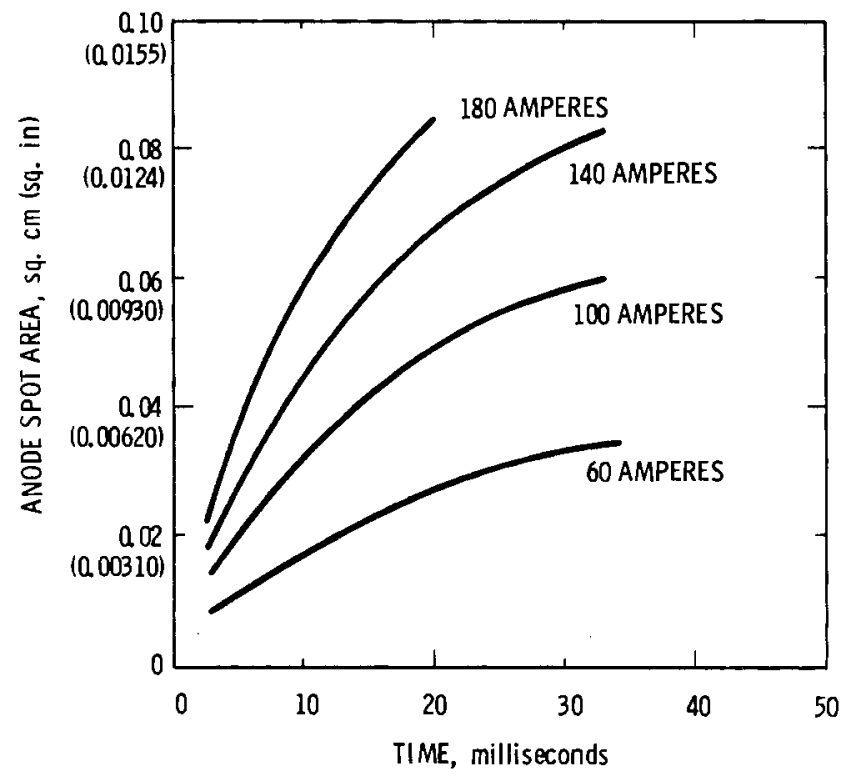
FIGURE 31.
Rate of Growth of Latera 1 Area
of Individual Anode Spots 61 


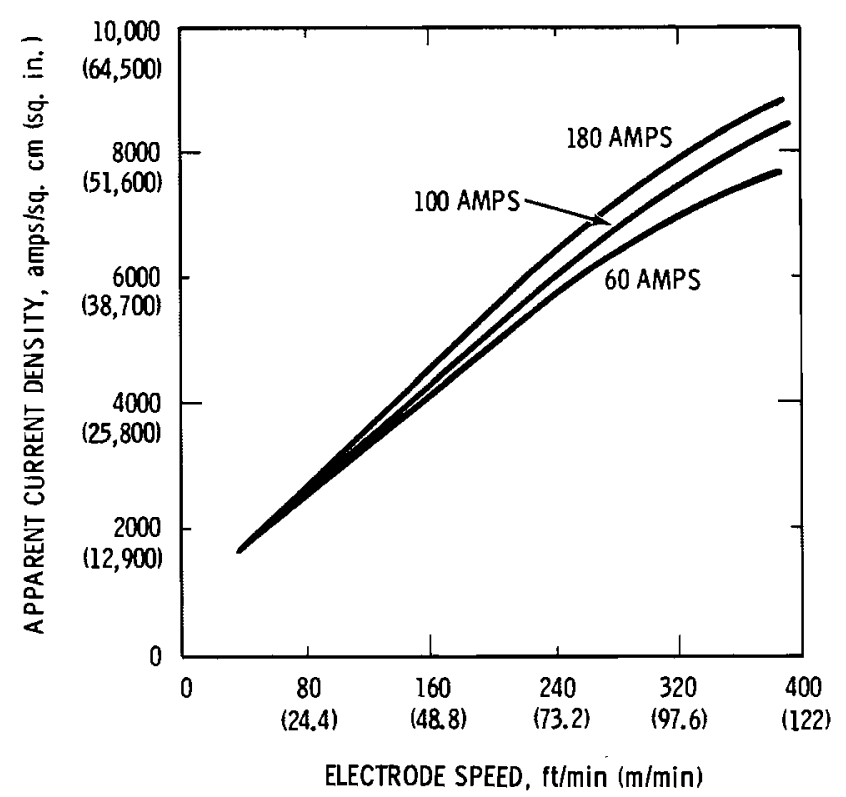

FIGURE 32. Variation of Apparent Current Density With Electrode Speed 61

Apparent current density variation with arc current at various electrode speeds is shown in Figure 33.61 These curves indicate that the apparent current density is approximately constant with current variation and a finite speed.

\section{Pressurizing Above Atmospheric Pressure}

A few papers have been published on the operation of welding arcs above atmospheric pressure. The principal work reported has been done on carbon steel. 62,63 A little work has been reported on Zircaloy-4 and beryllium. It is obvious that considerable work has been done on Zircaloy-2* and -4 * and not reported as the final end closure on nuclear fuel is being made at pressure above 20 bars $\left(200 \mathrm{~N} / \mathrm{cm}^{2}\right)$ helium. Definitive work has been done on carbon steel to 32 bars $^{63}\left(320 \mathrm{~N} / \mathrm{cm}^{2}\right)$, definitive work on beryllium to

\footnotetext{
*See Appendix C for compositions.
} 


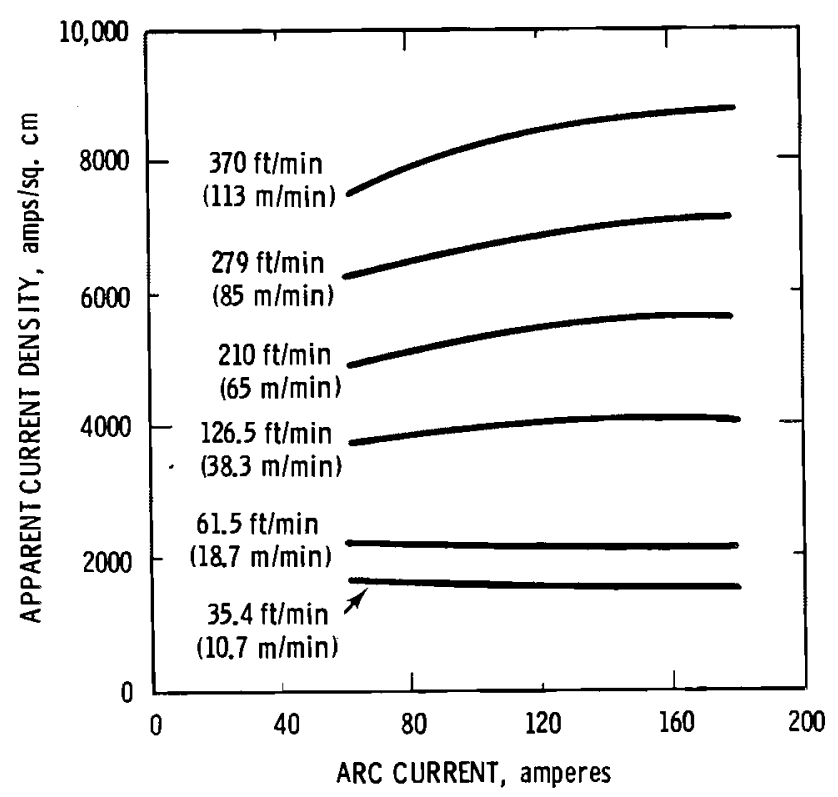

FIGURE 33. Variation of Apparent Current Density With Total Arc Current 61

4 bars $\left(40 \mathrm{~N} / \mathrm{cm}^{2}\right)^{64}$ and sketchy incomplete non-definitive notes on Zircaloy-4 to 40 bars $^{65}\left(400 \mathrm{~N} / \mathrm{cm}^{2}\right)$. In Figure $34^{65}$ is shown the effect of varying the electrode spacing at 20 bars $\left(200 \mathrm{~N} / \mathrm{cm}^{2}\right)$ on arc voltage while Figure $35^{65}$ shows the effect of varying the pressure on arc voltage for shielding gases of helium, 70/30 neon-helium and argon. In both cases Zircaloy-4 was being welded.

As the pressure of argon and helium shielding gases used for inert gas tungsten-arc welding increases above atmospheric to 40 bars (with the current, arc length, and travel speed held constant), the following take place:

- The arc voltage rises

- The arc column contracts

- The area covered by the arc on the cathode decreases raising the temperature of the electrode tip 


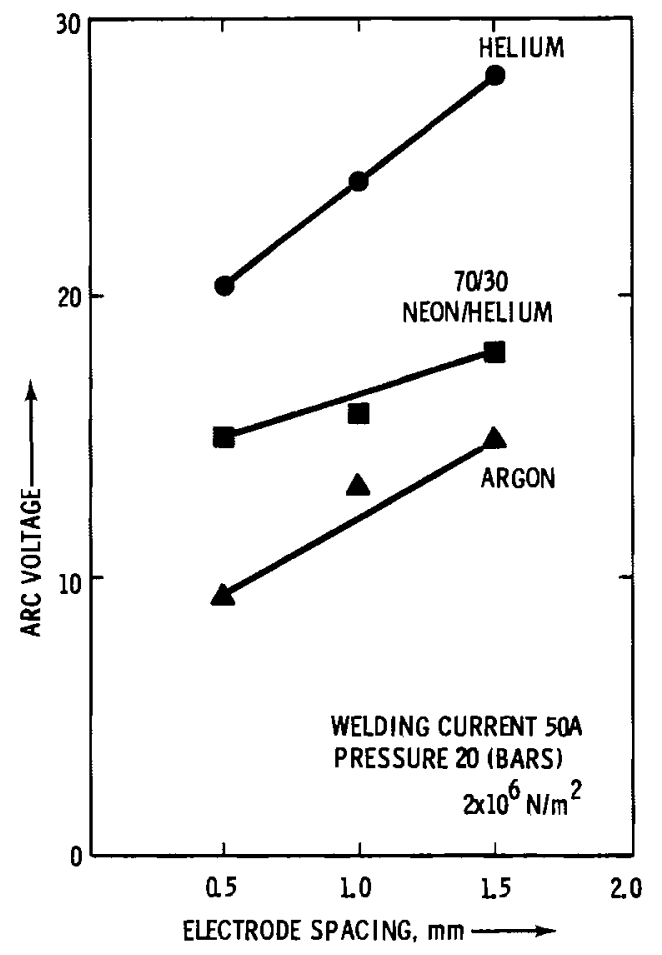

FIGURE 34. Electrode Spacing-Arc Voltage Characteristics 65

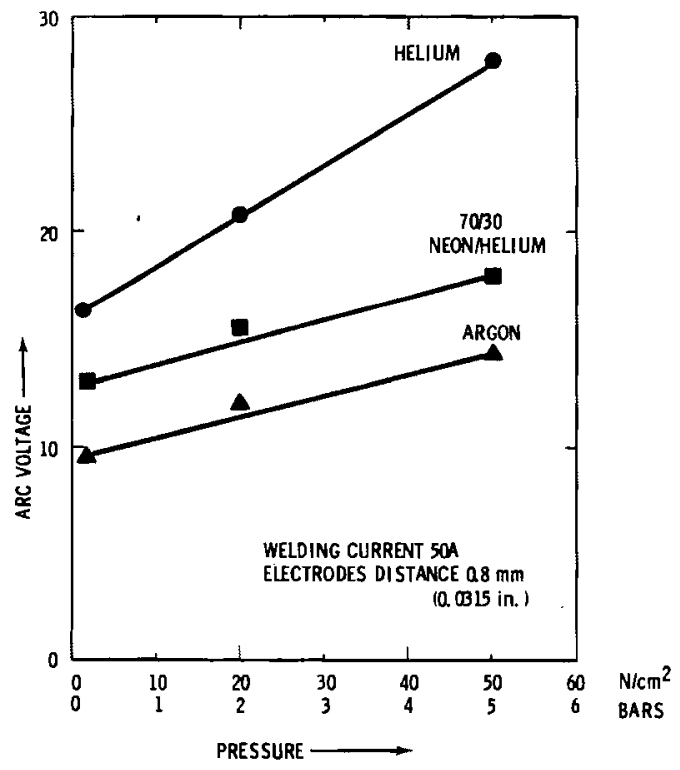

FIGURE 35. Gas Pressure-Arc Voltage Characteristics 65 
- The thoriated tungsten electrode tip melts and balls, with the cathode spot wandering around the ball, causing the anode spot to wander. 63

- The arc on carbon steel in helium becomes unstable at approximately 8 bars $\left(80 \mathrm{~N} / \mathrm{cm}^{2}\right)$; in argon at approximately 16 bars $\left(160 \mathrm{~N} / \mathrm{cm}^{2}\right) .63$

- Penetration increases with pressure until the thoriated tungsten electrode tip starts to melt, causing arc wander on both electrodes and decreasing penetration. 63,64

- Arc starting with standard high frequency and 80 volts open-circuit

- becomes impossible in helium at 2 bars $\left(20 \mathrm{~N} / \mathrm{cm}^{2}\right.$ ) and in argon at 4 bars $\left(40 \mathrm{~N} / \mathrm{cm}^{2}\right)$.

- In helium at 20 bars, $\left(200 \mathrm{~N} / \mathrm{cm}^{2}\right) 400$ open-circuit volts are required to start the arc, and 30 to 40 kilovolts are required for the high frequency starter. 65 There is some evidence that the arc will start in hel ium above 20 bars $\left(200 \mathrm{~N} / \mathrm{cm}^{2}\right)$ at 150 open-circuit volts with an impulse arc starter.

- When welding Zircaloy-4 in helium at 10 bars $\left(100 \mathrm{~N} / \mathrm{cm}^{2}\right)$ and above, metal vapors pass out of the arc in the form of a cloud. ${ }^{64}$ This was not reported with carbon steel. Composition of the deposit was $60 \mathrm{wt} \% \mathrm{Sn}, 30 \mathrm{wt} \% \mathrm{Zr}$, and $10 \mathrm{wt} \% \mathrm{C}$. Condensation and alloying of the metal vapors on the tungsten electrode tip hinders arc starting, requiring frequent electrode replacements.

- Damage to the thoriated tungsten cathode became visible in helium at 8 bars $\left(80 \mathrm{~N} / \mathrm{cm}^{2}\right)$ and in argon at 16 bars $\left(160 \mathrm{~N} / \mathrm{cm}^{2}\right) .62$

- The addition of cold filler wire to carbon steel welding stabilized the arc to 32 bars $\left(320 \mathrm{~N} / \mathrm{cm}^{2}\right)$, the maximum pressure investigated. 63 Gas metal arc welding produced satisfactory arc performance and welds to 32 bars $\left(320 \mathrm{~N} / \mathrm{cm}^{2}\right)$ using argon-oxygen mixtures on carbon steel. At 32 bars $\left(320 \mathrm{~N} / \mathrm{cm}^{2}\right)$ the brown fume coming from the arc was sufficient to prevent detailed viewing of the arc. ${ }^{63}$ As the gas pressure rose, the arc voltage increased. Shielded metal arc welding produced satisfactory arc performance and sound welds. As the gas pressure rose, the minimum arc voltage remained essentially constant. ${ }^{66}$ 


\section{ALTERNAT ING-CURRENT WELDING ARCS}

1. Influence on Arc Characteristics

The characteristics of an alternating-current arc are influenced markedly by:

- Nature of the electrodes

- Mass of the electrodes

- Shielding gas

- Shielding gas pressure

- Length of the arc

- Open-circuit voltage

- Current wave shape

- Constants of the current carrying circuit

2. Sinusoidal Current Arc Characteristics

Essentially, the instantaneous characteristics of the alternatingcurrent arc are equivalent to a direct-current arc of the same polarity.* Figure 36 shows the current and voltage wave forms of a balanced ${ }^{\star *}$ sinusoidal current welding arc with 5 argon -7 helium volume ratio shielding gas, a tungsten electrode, and an aluminum workpiece. Numbers 1 , 2 , and 3 indicate open-circuit voltages of 150, 100, and 80 volts, 4 indicates the arc voltage, and 5 the welding current which is in phase with it. The restrike voltage when the aluminum is negative is 6 . It will be noted that the magnitude of the restrike is greater than the instantaneous voltage of the 80-volt open circuit wave so that the arc will not restrike on an 80-volt power supply. However, by the application of superimposed high-frequency, pulse injectors, ${ }^{\star \star \star}$ a higher open-circuit voltage, or with welding current balancing capacitors, the necessary instantaneous voltage will be supplied so that the arc will continue to burn. A 5-7 volume ratio argon-helium arc will start and burn on aluminum with balancing of the current and without stabilization at 100 volts open-circuit at high values

*For a detailed discussion of the sinusoidal alternating-current arc, see Reference 127.

$\star \star$ For a discussion of the balanced current arc, see Appendix $D$.

$\star \star \star$ See Supplemental Reference 21. 


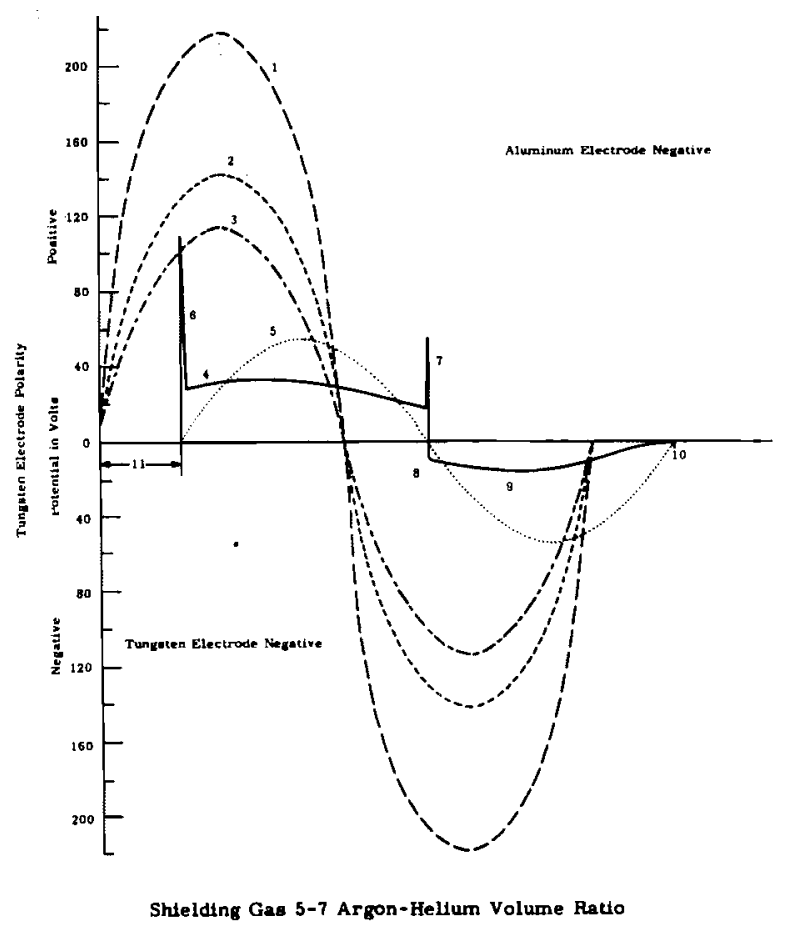

FIGURE 36. Voltage-Current Relationships for 200 Ampere Balanced Sine Wave Inert Arc

of current for the electrode used. Energy stored in the molten aluminum cathode pool when it is the anode plus the net energy added by the negative end of the arc* maintains the electron emission of the aluminum high enough that the open-circuit voltage plus the series current balancing capacitor voltage are not exceeded during the half-cycle. When the molten aluminum pool goes negative, the arc will go out or become unstable if the current is decreased. For crater filling, the required restrike voltage is greater than the algebraic sum of the instantaneous values of the open-circuit voltage and the series capacitor voltage. As the current decays during crater filling, the charge on the balancing capacitor decreases, thus decreasing its maximum voltage. A point is reached at which the restrike voltage (open-circuit voltage plus capacitor voltage) is no longer of sufficient magnitude when the tungsten electrode is positive and the arc does not restrike. When the tungsten electrode again becomes negative on *(Electron emission removes energy from the molten aluminum cathode pool and positive ion bombardment adds energy.) 
the second half cycle and the arc burns, a second charge is placed on the series capacitor which effectively doubles its voltage. The next time the tungsten electrode becomes positive there is sufficient restrike-voltage available to restrike the arc. During the arc burning period the charge on the series capacitor decreases, thus decreasing its voltage. The phenomenon repeats every two cycles or until the current has decayed to the point that the total charge on the capacitor plus the open circuit voltage is no longer large enough to produce the required restrike voltage. The weld surface is very rough and the weld has virtualiy no penetration.

An open-circuit potential of at least 150 volts is required when welding aluminum using argon shielding gas with a sine wave power supply with either balanced or unbalanced current and without high-frequency or surge-injector stabilization. The arc burning voltage, 4, in Figure 36 has a rising characteristic starting at the conversion from a cold cathode discharge to an arc. At this point the temperature of both the plasma and the cathode are forced to rise to supply and carry the increased number of electrons. As the current approaches its maximum value, the burning voltage increases more slowly and actually decreases before the maximum value of current is reached. With decreasing current there is excess heat in the electrode and plasma and the burning voltage drops lower than in the first quarter cycle. At the point where the instantaneous value of the open-circuit potential is no longer high enough to maintain thermionic emission, the arc changes to a cold cathode discharge. The extinguishing voltage, 7 , then appears. With the current reversing, the tungsten electrode is hotter than is required to supply the electrons. The electrode positive discharge starts as a true arc without evidence of the re-ignition voltage spike, 8 . In the sequence of the oscillograms from which this cycle was taken, a few restrikes showed evidence of an incipient spike. The burning voltage of the arc, 9, with the tungsten electrode negative is much lower than when the aluminum was negative. The tungsten is a much better electron emitter for the same value of current. The rate of rise of voltage after restrike and the maximum value are virtualiy the same for each half cycle. With decreasing current, the voltage decreases at about the same rate in each half cycle. Finally a point is reached where there is an abrupt change and the rate 
is decreased by almost one-half. As the current approaches zero, the arc voltage approaches zero eventually reaching the point at which the voltage starts to rise, 10, when the arc converts to a cold cathode discharge. When the current becomes zero the voltage becomes zero.

Other investigators agree that circuit elements, including the arc, develop stored energy and produce inductive kicks in voltage when the arc current approaches and passes through zero. Energy stored in circuit elements causes increases in voltage, for example, across inductances and capacitances which become manifest when the current goes through zero. The reason for the presence or absence of a spike on a series of half cycles when the tungsten is positive probably lies in the changes with time of circuit elements.

In Figure 36 the phase shift between the welding current and the opencircuit voltage is shown by 11 . Its value is determined by the quadrature relationship between the total resistance, inductance, and capacitance related to the welding current circuit. The displacement shown is not an actual case but was estimated by what is known of the characteristics of 80- and 100-volt open-circuit units. If there were virtually no resistance in the circuit and the value of the capacitance was very high (very low capacitive reactance), the restriking would occur at the maximum of the open-circuit voltage wave. Conversely, if resistance only was in the circuit, the restrike would not occur at the zero point of the open-circuit voltage wave. Since there is not adequate voltage, the arc would not restrike. However, since the current and voltage go through zero simultaneously, if the electrodes had sufficient thermal storage, the arc would be able to restrike at a later point in the half cycle. In addition, the smaller the ratio between the arc burning voltage and the open-circuit voltage, the nearer the maximum value of the open-circuit voltage approaches the restrike point.

\section{Half Cycle Polarity Reversal}

Upon polarity reversal of the alternating-current arc, depending on the electrode materials, the new cathode may be at a higher temperature than the previous cathode. Thus, it appears that a thermionic cathode can 
be established before appreciable de-ionization of the column takes place. This is particularly true for the refractory materials such as tungsten and carbon. It is the result of the anode voltage drop being greater than the cathode drop. ${ }^{67,68}$

The restriking of an alternating-current arc may be considered a race between 1) the de-ionization and electrode cooling, and 2) the rise in restrike voltage. The fleetest is the winner in determining whether the arc restrikes, becomes unstable, or goes out. ${ }^{69}$

High speed motion pictures ( 3000 frames/sec) disclose that the major part of the cathodic etching (cleaning action) in alternating-current gas tungsten arc welding aluminum takes place while the electrode positive current pulse is rising to its maximum value.

\section{Arc Ignition Characteristics}

In initiating the alternating-current inert-gas-shielded tungsten-arc, the first few cycles are composed of cold cathode, field emission, and thermionic discharges that form randomly over the end and shank when the tungsten electrode is negative as well as a fairly large area of the workpiece when it is negative (Figure 37). The point or points of arcing are determined by the lowest sparking potential. This is controlled by the natura 1 ionization* around the electrodes. In many instances, the initial sparking distance may be greater than the shortest distance between the electrodes. As the temperature of the electrode rises, the work function of the electrons in the tungsten decreases. The shielding gas temperature rise facilitates ionization by collision, the current increases, and the voltage decreases until the arc becomes stable with full operating temperature.

The number of cycles required to produce a stable arc is a function of:

- Shielding gas

- Shielding gas pressure

- Electrode material

*For a description, see Appendix E. 

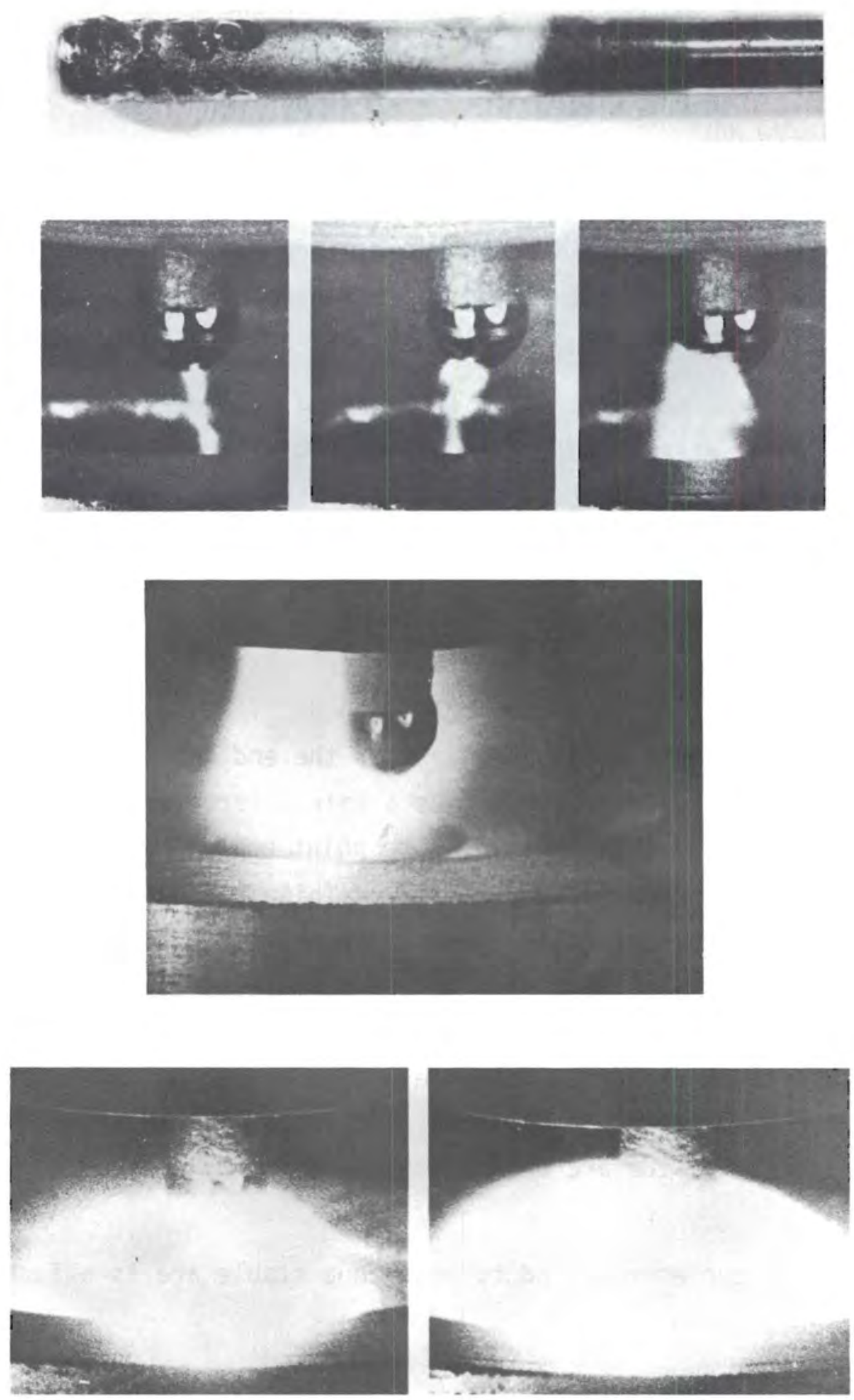

FIGURE 37. Sequence of Alternating-Current Arc Starting Sequence, Damage Done to Electrode by Arc Striking Shank, and Cathodic Etching of Shank 
- Mass of electrodes

- Open-circuit voltage

- Current magnitude

- Current wave shape of the power supply

- Constants of the current carrying circuit (R, L, C)

- Polarity and point on the voltage wave at which the arc is first ignited.

The volt-ampere characteristics of the argon-gas-shielded, alternatingcurrent balanced sine wave argon arc are shown in Figure 38 . It has virtually the same shape as the one for the direct-current arc.

5. Arc Hysteresis

In the alternating-current arc there is considerable difference in the volt-ampere characteristic of the arc between rising and falling currents. This difference is produced by the thermal lags of the electrodes and plasma. The high thermal lags assist in restriking the arc when the voltage

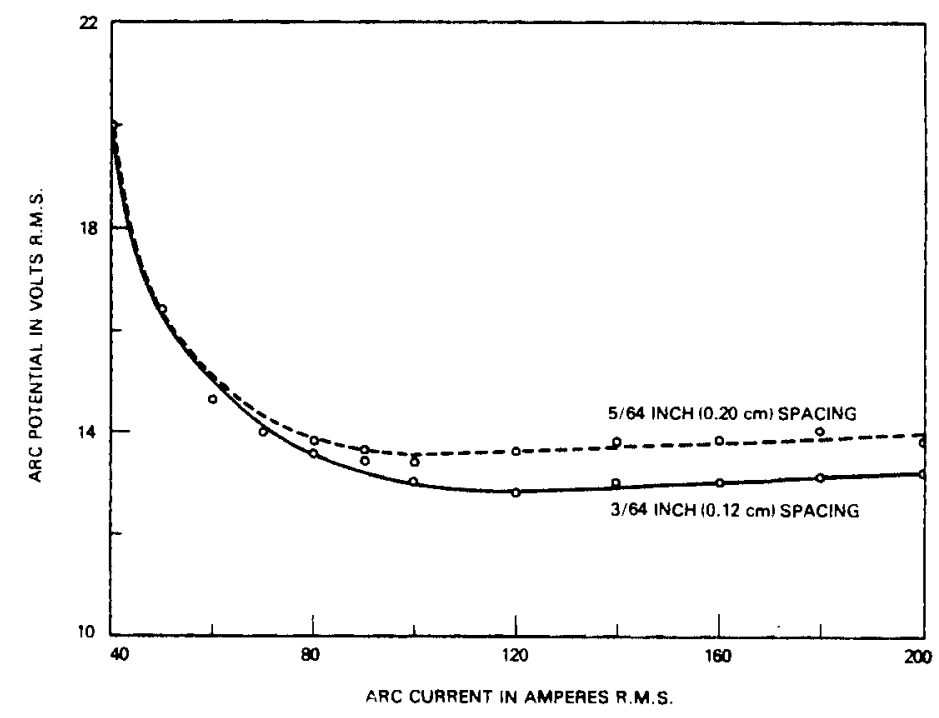

FIGURE 38. Volt-Ampere Characteristics of Alternating-Current Balanced Sine Waie Argon Arc. Argon Shielding, 1/8" (3.2mm) Tungsten Electrode, Aluminum Work Piece 
reverses. However, they also limit the rate at which an arc can be struck and stabilized. This is known as arc hysteresis 70,71 and is shown in Figure 39 for an arc with a balanced sine wave current, 5 argon - 7 helium volume ratio shielding gas, a tungsten electrode, and an aluminum workpiece. As the current goes to zero, the tungsten electrode is hotter than necessary for the required electron emission. Thus, very little restrike voltage (1) is required to re-ignite the arc as the tungsten electrode goes negative. Owing to the de-ionization and cooling taking place during the period of zero current and the rising current requiring more electrons, the burning voltage of the arc (2) during the period of increasing current will be greater than the static characteristic value (3). After the current maximum is reached, ionization of the shielding gas and electron emission of the thoriated tungsten electrode are in excess of that required for lower currents. Therefore, for decreasing currents the voltage will be less than the static value (4). At low currents the voltage rises with decreasing

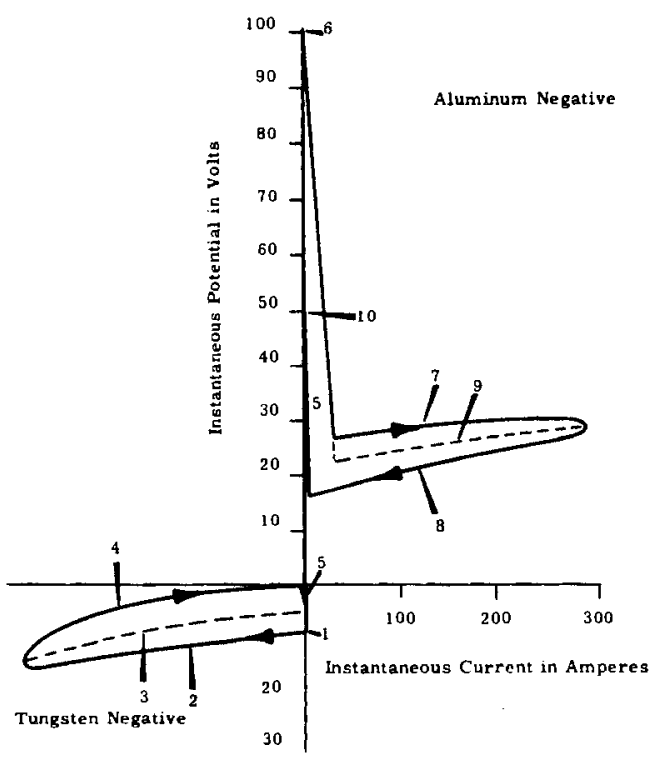

Shielding Gas 5-7 Argon-Helium volume Ratio

FIGURE 39. Arc Hysteresis Loop for 200 Ampere Balanced Sine Wave Inert Arc 
current (as it would in the static case) reaching a maximum at the extinguishing voltage is dependent on whether there is enough voltage to maintain the arc discharge as the current approaches zero or the arc will go out.

As the current goes to zero, de-ionization of the gas and very fast cooling of the aluminum electrode take place reducing the available electrons. As a consequence a very high value of restrike voltage (6) is required to re-ignite the arc when the aluminum electrode goes negative. The low thermal emission characteristic and high thermal conductivity of aluminum produce, with increasing current, a burning voltage (7) approximately three times that of when the tungsten electrode is negative. After the current maximum is reached, ionization of the shielding gas and electron emission of the aluminum are in excess of that required for lower currents. Therefore, for decreasing current the voltage (8) will be less than the static voltage (9). At low currents the voltage rises and reaches a maximum at the extinguishing voltage (10) as the arc changes to a field emission or possibly cold cathode discharge before being extinguished. In going through zero there is a very short interruption in the current, detectable by oscillography, the length of which is determined by the current wave shape, open-circuit voltage, and electrode materials and mass.

\section{Effect of Cathode Substances on Restriking}

Cathode substances fall into two groups when the restriking time is investigated: a circuit containing a carbon arc can be interrupted for up to about one second at open-circuit potentials of several hundred volts and will restrike without a change of the electrode separation. A copper arc when interrupted for $10^{-3}$ seconds or less will not restrike. ${ }^{72}$

\section{Effect of Travel Speed on Arc Stability}

With a constant-current alternating-current arc, the point of instability of the arc as the travel speed increases is determined by the cathode spot on the workpiece as it is more sensitive to temperature change than the anode. However, the point of instability can be extended to higher speed by increasing the open-circuit voltage across the arc, Table 2. ${ }^{125}$ 
TABLE 2. Square Current Wave, Arc Stability Relationships For Variable Gas Ratios 125

\begin{tabular}{|c|c|c|c|c|c|c|c|c|c|c|c|c|}
\hline \multirow{4}{*}{$\begin{array}{l}\text { OPEN } \\
\text { CIRCUIT, } \\
\text { VOLTS }\end{array}$} & \multirow{3}{*}{\multicolumn{2}{|c|}{$\begin{array}{l}\text { SHIELDING GAS } \\
\text { VOLUME RATIO } \\
\end{array}$}} & \multirow{3}{*}{\multicolumn{2}{|c|}{$\begin{array}{c}\text { HIGH } \\
\text { FREQUENCY } \\
\text { STABILIZATION } \\
\end{array}$}} & \multicolumn{8}{|c|}{ ARC STAB ILITY, inches/min (cm/min) } \\
\hline & & & & & \multicolumn{4}{|c|}{$17.8(45.2)$} & \multicolumn{4}{|c|}{$52.9(134)$} \\
\hline & & & & & & ALMOST & & VERY & & ALMOST & & VERY \\
\hline & ARGON & HELIUM & YES & No & UNSTABLE & STABLE & STABLE & STABLE & UNSTABLE & STABLE & STABLE & STABLE \\
\hline 80 & $\left\{\begin{array}{l}x \\
x \\
5\end{array}\right.$ & 7 & $\begin{array}{l}x \\
x\end{array}$ & $x$ & $\begin{array}{l}x \\
x\end{array}$ & & & $x$ & $\begin{array}{l}x \\
x\end{array}$ & & & \\
\hline 90 & $\mid \begin{array}{l}x \\
5\end{array}$ & 7 & $\mathrm{x}$ & $\mathrm{x}$ & $x$ & & & & $x$ & & & \\
\hline 95 & $\mid \begin{array}{l}x \\
5\end{array}$ & 7 & $\mathrm{x}$ & $\mathrm{x}$ & & & $x$ & & & $x$ & & \\
\hline 100 & $\mid \begin{array}{l}x \\
x \\
5\end{array}$ & 7 & $\begin{array}{l}x \\
x\end{array}$ & $x$ & & & $x$ & & & $x$ & $x$ & \\
\hline 105 & $\left\{\begin{array}{l}x \\
5 \\
5\end{array}\right.$ & $\begin{array}{l}7 \\
7\end{array}$ & $\mathrm{x}$ & $\begin{array}{l}x \\
x\end{array}$ & $x$ & & $x$ & & & & & \\
\hline 110 & $\mid \begin{array}{l}5 \\
3\end{array}$ & $\begin{array}{l}7 \\
9\end{array}$ & & $\begin{array}{l}x \\
x\end{array}$ & $\mathrm{x}$ & & $\mathrm{x}$ & & & & & \\
\hline 120 & $\mid \begin{array}{l}5 \\
3\end{array}$ & $\begin{array}{l}7 \\
9\end{array}$ & & $\begin{array}{l}x \\
x\end{array}$ & & & & & $x$ & $x$ & & \\
\hline 125 & $\mid \begin{array}{l}5 \\
3\end{array}$ & $\begin{array}{l}7 \\
9\end{array}$ & & $\begin{array}{l}x \\
x\end{array}$ & & & & & $\mathrm{x}$ & & $x$ & \\
\hline 130 & $\begin{array}{l}5 \\
3\end{array}$ & $\begin{array}{l}7 \\
9\end{array}$ & & $\begin{array}{l}x \\
x\end{array}$ & & & & & & & & $\begin{array}{l}x \\
x\end{array}$ \\
\hline
\end{tabular}




\section{HEAT TRANSFER OF DIRECT-CURRENT WELDING ARCS}

\section{A. HEAT TRANSFER WITHIN ARC* ${ }^{73}$ \\ 1. Transport Properties Within Arc}

As the attained temperatures and degree of thermal ionization are increased the effects of neutral molecules and atoms contained in the arc plasma are lessened because of a decrease in their number density. On the other hand, there is a proportionate increase in the number density of ions and electrons which take on the character of the transport properties of the plasma as the temperature rises. Moreover, the coupling of ions and free electrons to the self-magnetic field introduces pressure gradients. These gradients, which produce acceleration of the particles, cause streaming toward either terminal of the arc. The irreversible transformation of input energy in arcs is controlled by the intensity and distribution of these forces and fluxes as influenced by the composition of the arc plasma. Several gases have been chosen to illustrate how their transport properties influence the geometric forms of arc plasma exhibited.

\section{Plasma Composition}

Molecular dissociation, an energy process to which plural atomic gases are subjected, is shown in Figure 40 for hydrogen, ${ }^{74}$ nitrogen, ${ }^{77}$ and carbon dioxide. ${ }^{75}$ Molecular dissociation is essentially compiete for all three gases at $10,000^{\circ} \mathrm{K}\left(18,000^{\circ} \mathrm{F}\right)$. Particle number densities as related to temperature for argon, nitrogen, and helium are shown in Figures 41,42 , and 43 . The determination of the particle-number densities was made according to the Saha theory of the thermal dissociation and ionization. ${ }^{76}$ The ordinate is given in units of particle number per $\mathrm{cm}^{3}\left(\mathrm{in}^{3}\right)$. The temperature range (abscissa) covers that involved in most welding and cutting arcs.

*This section reproduces virtually all of an article by $H$. C. Ludwig "Plasma-Energy Transfer in Gas-Shielded Welding Arcs," Welding Journal, Welding Research Supplement, Volume 38, pp. 296s-300s, 1959. In association with the Metals Joining Section of the Westinghouse Research Laboratories, Pittsburgh, the author presented the paper at the 1958 American Welding Society Annual Spring Meeting in St. Louis, April 14-18. The article is reproduced herein with the permission of the American Welding Society. 


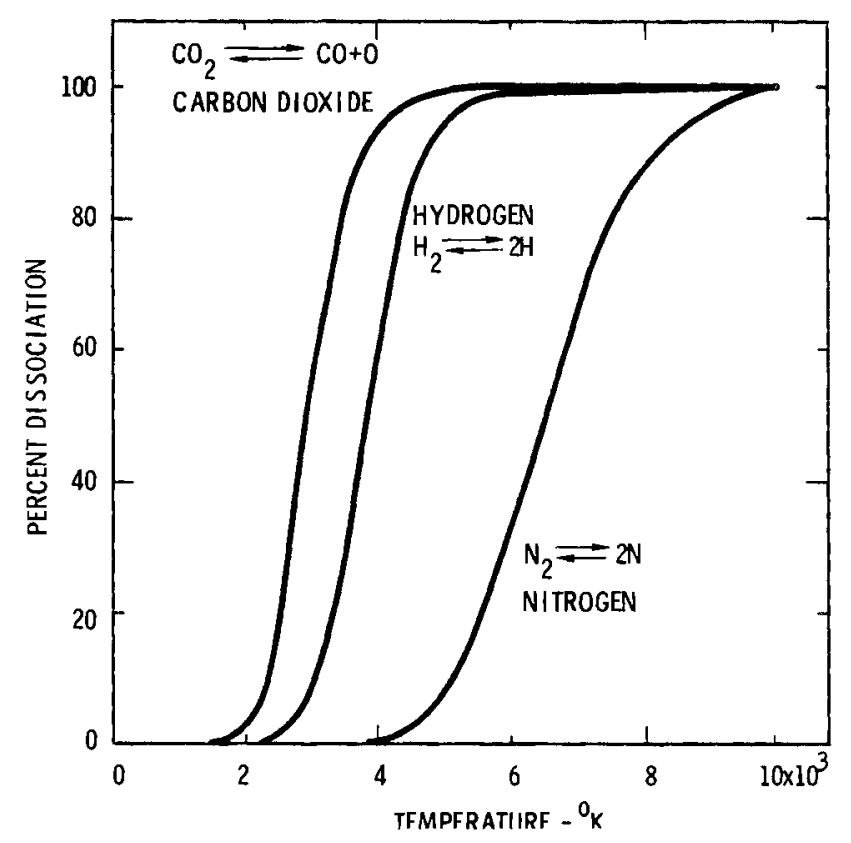

FIGURE 40. Dissociation in Molecular Gases at $1 \mathrm{~atm}$ Pressure ${ }^{73}$

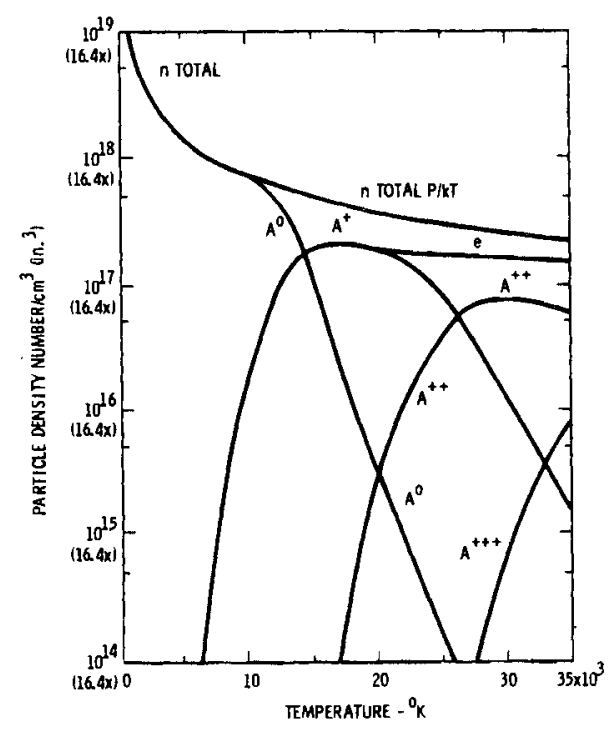

FIGURE 41. Argon-Shielded Arc Plasma Composition $760 \mathrm{~mm}$ (14.7 PSI) Pressure, G. Busz and W. Finkelburg ${ }^{73}$ 


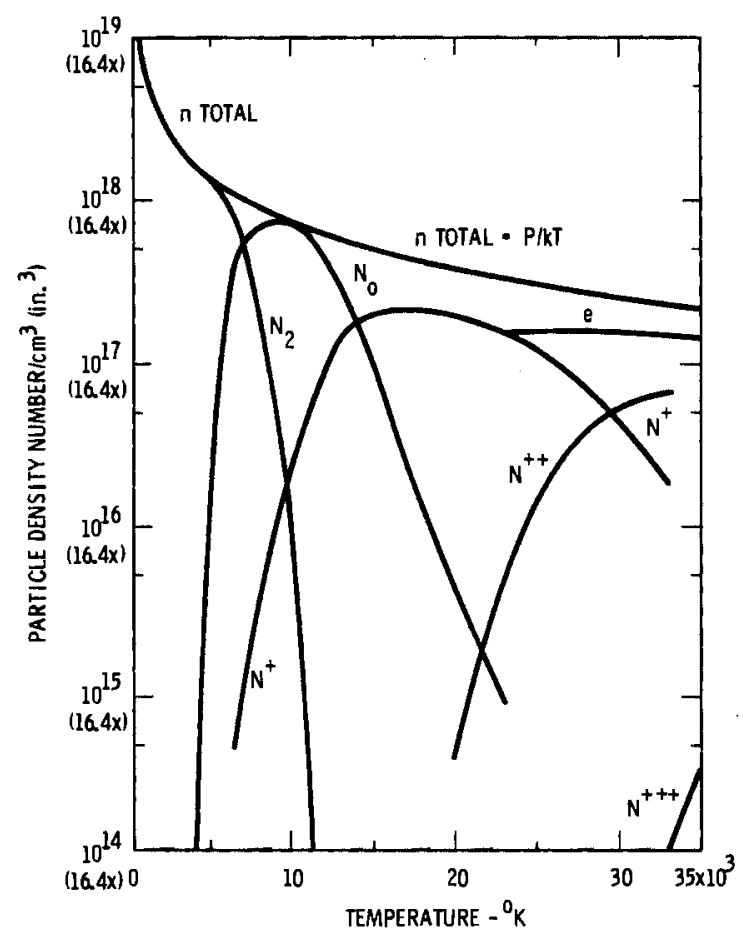

FIGURE 42. Nitrogen-Shielded-Arc Plasma Composition $760 \mathrm{~mm}$ (14.7 PSI) Pressure, G. Busz and W. Finkelburg ${ }^{73}$

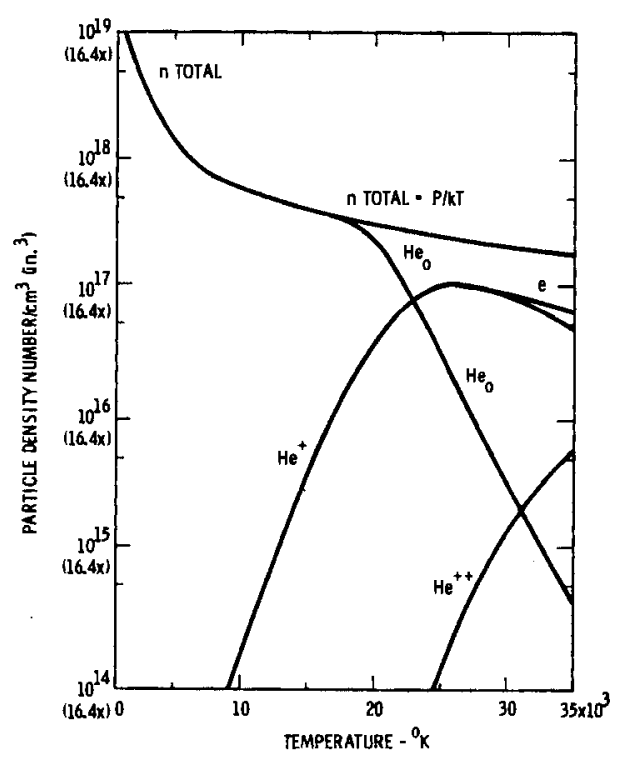

FIGURE 43. Helium-Shielded Arc Plasma Composition $760 \mathrm{~mm}$ (14.7 PSI) Pressure 73 
In following the course of dissociation and thermal ionization with temperature (Figures $41,42,43$ ), one starts at the upper left hand corner of each semi-logarithmic graph corresponding to the maximum total number of atoms or molecules per $\mathrm{cm}^{3}\left(\right.$ in. $\left.^{3}\right)$, the total being some $10^{19}$ particles per $\mathrm{cm}^{3}\left(6.62 \times 10^{19}\right.$ particles/in. $\left.{ }^{3}\right)$ at room temperature. As the temperature increases, molecular dissociation may take place as for nitrogen (Figs. $40^{73}$ and $42^{73}$ ), and excitation and ionization proceed. The total number of particles per unit volume varies with temperature as shown by the topmost curve in each figure. The density of singly ionized atoms, for example $\left(\mathrm{N}^{+}\right)$, increases with temperature and reaches a maximum, while the density of neutral atoms $\left(N_{0}\right)$ decreases. The increase in the density of doubly ionized particles occurs at approximately $17,000^{\circ} \mathrm{K}\left(30,600^{\circ} \mathrm{F}\right)$ for argon and nitrogen and $26,000^{\circ} \mathrm{K}\left(46,800^{\circ} \mathrm{F}\right)$ for helium. The curves for electron density (e) follow the low temperature side of singly ionized particles to their maxima and thereafter follow separate curves as the singly ionized particle densities decrease. Each stage of ionization contributes to the number of free electrons. The particle-density curves for argon and nitrogen are from G. Busz and W. Finkelnburg, 77 and the curve for helium from H. C. Ludwig.

\section{Arc Energy Transfer}

The electrical energy is changed, within the arc, into other forms of energy. An understanding of the details of these changes provides information about the thermal conductivity of the arc plasma. The usefulness of the arc as a joining tool is largely determined by the manner in which the electrical energy is transformed into heat energy; however, other forms of energy may extend this utility.

The heat energy in an arc is transferred by the mechanisms of:

- Conduction

- Convection

- Radiation

These are influenced to some extent by the development of a magnetic field, another form of energy. In the analysis of the arc energy, a division can be made into that portion which moves axially, governed primarily by 
Ohm's law, and that which moves radially, accounting for the deviation in electrical conductivity from Ohm's law. The irreversible process of energy transformation produces magnetic and thermal forces and fluxes which incite the movement of plasma particles and energy. Account is made for the total of these quantities in the magnitude of the plasma-field strength.

For definitive purposes, a description of the mechanism of heat energy transfer in a plasma is reviewed. Conduction takes place by means of interparticle transfer from a region of higher temperature, called the source, to a region of lower temperature, called the sink. Radiation travels in waves through gaseous and vacuous space. Convection of the natural type is driven by the force of gravity and depends on the difference in mass density of the heated gas and the main body of the surrounding gas. Diffusion which can take place via molecules, atoms, ions, and electrons, depends on both concentration and thermal gradients.

Radial heat transfer by conduction and diffusion mechanisms in arc discharges has been treated by Maecker ${ }^{78}$ in the following expression for the thermal-conductivity coefficient $(X)$ :

$$
x=\left[\frac{1 / 2 k v_{0} n_{0}}{4 \sqrt{2 Q_{0}\left(n_{0}+n_{+}\right)}}\right]+\left[\frac{\left[1 / 2 k n_{+}+2 / 3 V\left(d n_{+} / d T\right] v_{+}\right.}{4 \sqrt{2} n_{0} Q_{0}+1 / 4 n Q_{++}}\right]+\left[\frac{1 / 2 k v_{e} n_{e}}{n_{0} Q_{0}+5 / 4 n_{e} Q_{+}}\right]
$$

where

$$
\begin{aligned}
\mathrm{k} & =\text { Boltzman's constant } \\
\mathrm{v}_{0}, \mathrm{v}_{+}, \text {and } \mathrm{v}_{\mathrm{e}}= & \text { respective thermal velocities of atoms, ions, and } \\
& \text { electrons. } \\
\mathrm{n}_{0}, \mathrm{n}_{+}, \text {and } \mathrm{n}_{\mathrm{e}}= & \text { respective particle densities of atoms, ions, and } \\
& \text { electrons. } \\
\mathrm{Q}_{0} \text { and } \mathrm{Q}_{+} \quad & \text { effective cross sections for atoms and ions, } \\
& \text { respectively. } \\
\mathrm{v} & \text { ionization potential of the plasma gas. }
\end{aligned}
$$

It is possible to make similar calculations to account for the contributions of heat transfer due to the recombination of atoms to molecules if the diffusion coefficient differs from the normal atoms. As the temperature 
increases the contribution due to electrons becomes greater and, therefore, through classical electron conduction assumes the important role of thermal conductivity.

\section{Thermal Conductivity}

From the composition of plasma gases, obtained by means of the Saha equation, ${ }^{76}$ and the development of thermal velocities and effective cross sections by kinetic theory, one can determine the total thermal conductivity of gases. The most recent progress in the determination of cross sections has been compiled by Drawin. ${ }^{79}$ This compilation includes the results of Gvosdover, ${ }^{80}$ Maecker, ${ }^{78}$ Chapman and Cowling, 81 and Spitzer and Harm. 82 From these data and those aforementioned, the total thermal conductivity can be determined by adding all of the contributions due to molecules, atoms, ions, and electrons.

The total thermal and electrical conductivities of several gases has been determined. The coefficients for hydrogen, 83 helium and nitrogen, $84,85,86$ and argon $77,84,86,87$ are shown in Figures $14 \mathrm{~A}$ and $14 \mathrm{~B}$ for a wide temperature range. The curve for helium was obtained from calculations by $H$. C. Ludwig. A general increase in thermal conductivity with temperature for all gases is noted. Contributions of the molecular dissociation-diffusion-association mechanism in diatomic gases, hydrogen, and nitrogen are readily observed. In the following section an attempt will be made to show how the plasma transport property of the thermal conductivity influences the geometric shape of the arc discharge in argon and helium.

\section{Nature of High Current-Density Arcs}

Experimental evidence shows that thermal conduction influences the geometric shape of the arc discharge. To illustrate the point, a comparison is made of the geometric forms of arc plasma due to low- and high-thermal conductivity gases, argon, and helium, respectively. A series of accurately reproduced illustrations of electrode negative arcs burning in argon, helium, and argon-helium gas mixtures is shown in Figure $44 .^{73}$ In this figure the pure helium arc is shown on the left and the pure argon on the right. As helium is added to the argon arc the plasma is visibly altered by the 


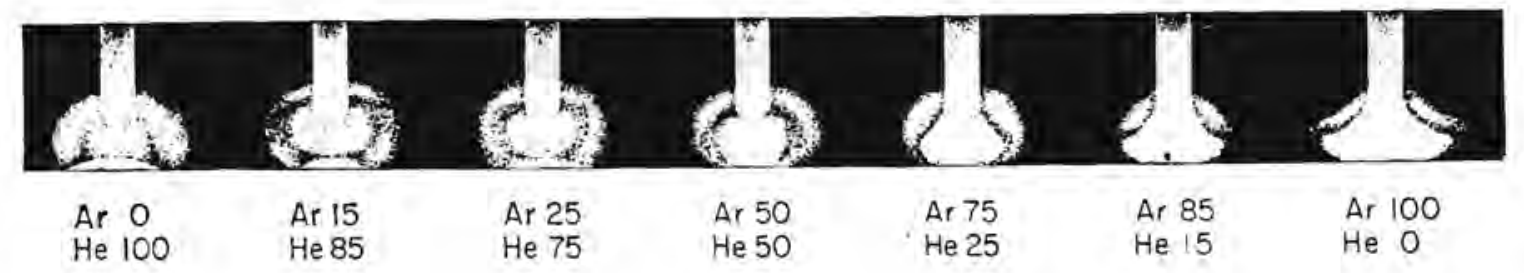

FIGURE 44. The Dependence of Arc-Plasma Shape on Composition (Percent by Volume) of Argon-Helium Gas-Shielded Arcs (Tungsten Electrode, 100 Ampere, Electrode Negative 73

gradual change from the truncated cone to a spherical form. A11 arcs were maintained at a current of $100 \mathrm{~A}$. The variation in the plasma gradient (field strength) with plasma composition is shown in Figure $45^{73}$ indicating a considerably higher power consumption for the helium arc than for the argon arc. The higher potential across the helium arc also indicates that higher temperatures are necessary and that more energy is being dissipated to maintain the discharge. Measurements of the radial temperature of an argon-tungsten arc by spectroscopic means show that the temperatures in the arc axis attain values on the order of $16,000^{\circ} \mathrm{K}\left(29,000^{\circ} \mathrm{F}\right)$ with the temperature falling to $10,000^{\circ} \mathrm{K}\left(18,000^{\circ} \mathrm{K}\right)$ near the boundary. A limited survey of temperature distribution in the helium arc indicates an axial temperature of $27,500^{\circ} \mathrm{K}\left(49,000^{\circ} \mathrm{K}\right)$. Shown in Figure $46^{73}$ are H. C. Ludwig's isotemperature curves for 300-A argon tungsten arc. 


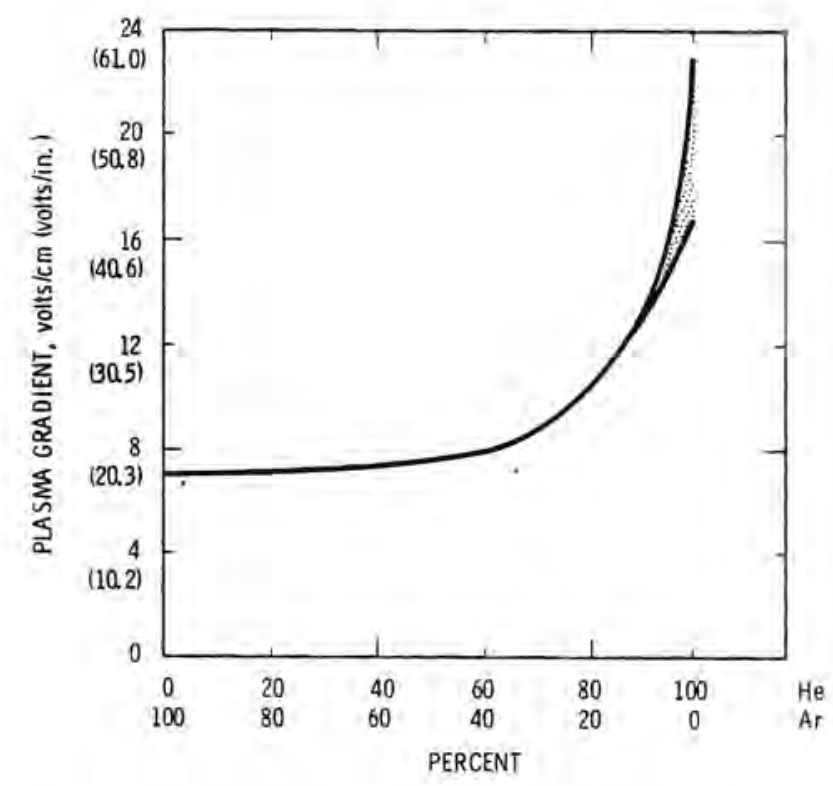

FIGURE 45. Dependence of Plasma Gradient on Composition (Percent by Volume) of Argon-HeTium Shielded Arcs. (Tungsten Electrode Negative 100 Ampere 73

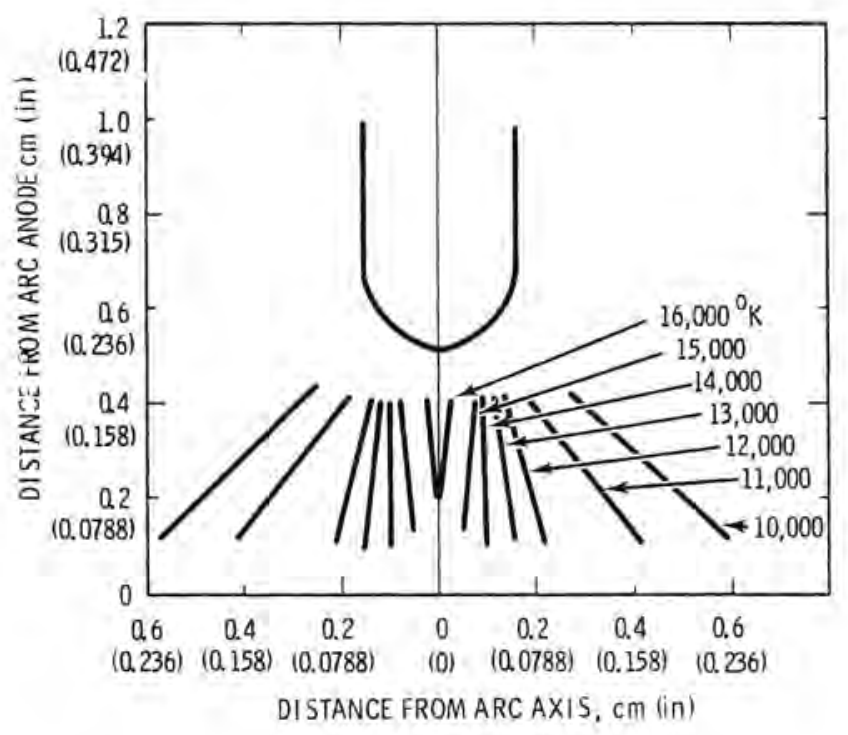

FIGURE 46. Temperature Distribution in an Argon Shielded Tungsten Arc 300 Amperes Electrode Negative 73 
If the thermal conductivity of a gas is very high, the plasma will be approximately isothermal. In other words, this means that the temperature gradient of a helium plasma will be significantly less than for the argon plasma. Also, the mean temperature for any given horizontal plane of the helium arc will be greater than that for a corresponding argon plasma because of both the higher axial temperature and lower temperature gradient. In a given volume the thermal gas pressure for the helium arc will be higher than for the argon, according to the Ideal Gas Law.

The interaction between the arc-plasma kinetic pressure and the selfmagnetic field pressure is a very important factor in physical characteristics of the arc as the current conducted becomes high. The amount of conducted current to make this interaction significant varies with the gas making up the arc plasma. The self-magnetic field effect of "pinch," the force acting to reduce the cross section of the conductor, has been observed in mercury vapor, ${ }^{88}$ xenon, ${ }^{89}$ argon, 90,91 and in carbon arcs. ${ }^{92} \mathrm{~A}$ streaming of the plasma particles which occurs away from the plasma zone of smallest cross section and of highest pressure has been observed with measured velocities as high as $300 \mathrm{~m} / \mathrm{sec}^{85}$ (984 ft/sec) (a speed approaching that of sound) and with developed forces on the order of 1000 dynes 90,92 $\left(2.248 \times 10^{-3} \mathrm{lbs}\right)$. The axial gradient in pressure introduced by the compressive force of the magnetic field produces the streaming motion which transfers metal through the arc.

The plasma pressure must be balanced by the combined magnetic pressure and atmospheric pressure. The effects of convection, conduction, diffusion, and radiation mechanisms contributing to the attained temperature of the arc plasma and temperature gradient should appear in a rigorous energybalance equation. An analogy in pressure balance may be found in the equilibrium of pressure within and without a soap bubble or balloon where atmospheric pressure and the pressure exerted by the bubble or balloon wall confine the gas. The arc plasma may also contain an axial gradient in pressure. This is analogous to the difference in pressure produced when a break or opening is made in the bubble or balloon causing the escape of the confined gas. 
In the diagrams of Figure $47, A^{73}$ and $B, 73$ a balance of the interacting forces $^{93}$ is illustrated consisting of 1) the kinetic pressure of the gas radial outward from the arc axis, and 2) the self-magnetic field pressure inward. The self-magnetic field varies with the distance $(r)$ from the axis of conducting radius (a) according to the relation: $H-2 i r / a^{2}$, where $i$ is the current in electromagnetic units. The magnetic force will be $2 \mathrm{ir} / \mathrm{a}^{2}$ at a distance $(r)$ from the axis directed tangentially to $(r)$ in a clockwise sense when the current passes into the plane of Figure 47A. 97 Considering a small element in the conductor $r d r d \theta$ ), the current flowing will be ( $r \mathrm{dr} d e i \pi \mathrm{a}^{2}$ ). The force upon this element over a length of $1 \mathrm{~cm}$ (0.394 in.) acting radially inward will be

$$
d F=\frac{2 i^{2}}{\pi a^{4}} r d r \cdot r d \theta
$$

The force will be supported by the small area $(r \mathrm{~d} \theta)$ so that the pressure will be

$$
d p=\frac{2 i^{2}}{\pi a^{4}} r \cdot d r
$$

The total area on the ring, as shown in Figure 47A, will be

$$
p=\frac{2 i^{2}}{\pi a^{4}} \quad \int_{r}^{a} r \cdot d r=\frac{i^{2}}{\pi a^{4}}\left(a^{2}-r^{2}\right) \frac{d y n}{c m^{2}}
$$

The total difference in pressure acting radially inward will be

$$
P_{\text {mag. }}=\frac{i^{2}}{\pi a^{2}}
$$

tending to oppose the kinetic pressure of the confined high temperature pìasma. 


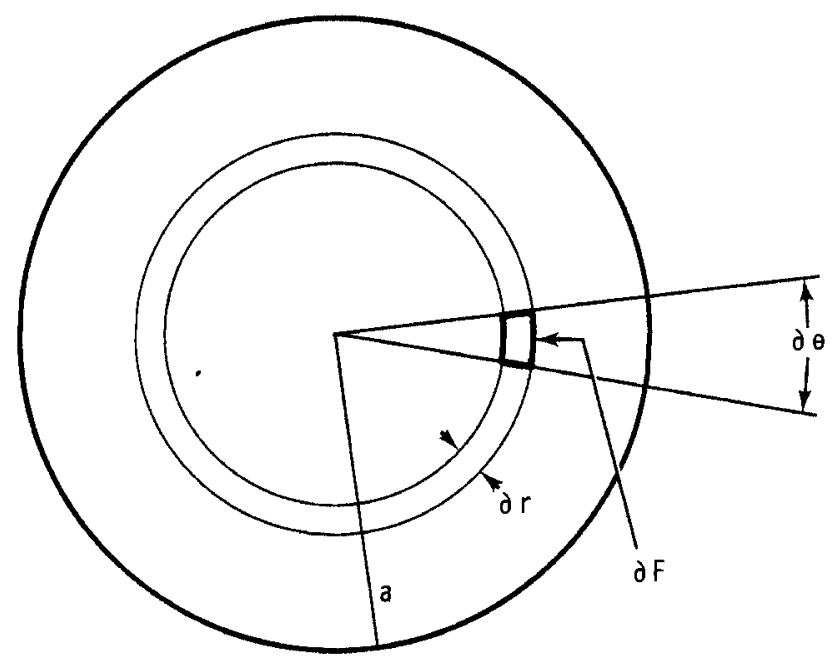

(a) $P_{\text {mag }}=\frac{i^{2}}{\pi a^{2}}$ dynes $/ \mathrm{cm}^{2}\left(1.405 \times 10^{-7} 0 z / \mathrm{in}^{2}\right)$

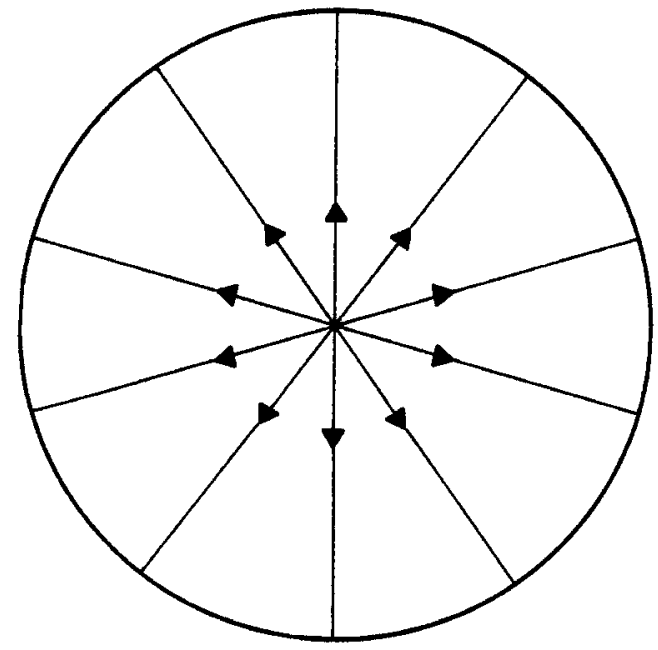

(b) P $P_{\text {therm. }}=\left(n_{+}+n_{e}+n_{0}\right) k T$

FIGURE 47, A \& B. Opposing Pressure of (A) Self Magnetic Compression and (B) Kinetic Gas Pressures in Arcs 73 
The kinetic pressure exerted radially outward by a plasma of infinite thermal conductivity would be, according to the Ideal Gas Law

$$
P_{\text {therm. }}=\left(n_{0}+n_{+}+n_{e}\right) k T
$$

where $n_{0}, n_{+}$, and $n_{e}$ are the representative gas particle densities, $k$ is Boltzman's constant, and $T$ is the plasma temperature, assuming an isothermal condition.

However, since welding arc plasmas have finite thermal conductivities, a radial gradient in temperature occurs. Therefore, the pressure of the gas will vary as a function of the temperature distribution. Equilibrium between the opposing forces is shown by the equation

$$
\operatorname{gradP}=\vec{J} \times \vec{H}
$$

where $\vec{J}$ is the current density and $\vec{H}$ is the magnetic field intensity. Gases which attain a higher temperature and lower temperature gradient will attain a higher pressure for a constant volume. The determination of the radial plasma pressure gradient which exists with a radial temperature gradient is mathematically complicated and will not be treated here. However, if we can assume equilibrium between the plasma pressure and the magnetic pressure, a solution can be more easily procured through the determination of the self-magnetic field pressure by integration of $\vec{J} \times \vec{H}$. It is apparent that kinetic pressure of the gas for a given volume is greater for higher temperature; thus the gas expands until the equilibrium with opposing pressures is attained. Because of both the higher axial temperature and lower temperature gradient, the hel ium-tungsten arcs are more difficult to "pinch," requiring a higher magnetic force. A higher magnetic force may be obtained by increasing the drawn arc current since the magnetic force varies as the square of the current. A threshold appears with the helium-tungsten arc at about a $150 \mathrm{~A}^{94}$ current where the shape of the arc changes from a sphere to a truncated cone. With a consumable steel electrode $[1.5 \mathrm{~mm}(0.0591 \mathrm{in.})]$ the threshold of discernable pinch occurs at $470 \mathrm{~A}$, reverse polarity. 
B. PLASMA ENERGY TRANSFER TO A SOLID $\operatorname{SURFACE}^{95}(*, * *)$

1. Plasma Heat Transfer Without Current Flow to an Anode

At first, heat transfer on a surface which is exposed to a high temperature gas stream but through which no electric current flows will be briefly reviewed. The heat transfer process has been studied extensively in recent years with reference to the reentry problem of space vehicles falling into the atmospheres of the earth or of other planets; the process was studied by research conducted analytically with results which have been verified by experiments in shock tubes and in arc wind tunnels. The gases considered were: air, nitrogen, carbon dioxide, and argon.

The pressure range in which the heat transfer to the skin of the vehicle becomes crucial is such that thermal equilibrium (among translation, vibration, excitation, and electron temperatures) is closely approximated; it is also such that the process can be described by continuum analysis. The re-entering object has generally a blunt shape, and maximum heat transfer occurs near the stagnation point.

When the entry velocity is sufficiently large, the object is surrounded by a layer which has been heated to temperatures causing a molecular gas to dissociate. The surface itself has to be kept cold; it is therefore surrounded by a usually laminar boundary layer within which the temperature drops to the surface value. Recombination of atoms consequently occurs, either within the boundary layer or by catalytic action at the surface of the skin of the vehicle. Energy is transferred through the boundary layer

\footnotetext{
*Application of this data is limited to heat transfer to a water cooled anode, a working fluid of argon and pressures greater than atmospheric. **This section reproduces virtually all of an article by $E$. R. G. Eckert and E. Pfender - "Plasma Energy Transfer to a Surface With and Without Electric Current," Welding Journa1, Welding Research Supplement, Volume 46, pp 471s-480s, 1967. The article is a review of the authors' work sponsored by the NASA Lewis Research Center under Contract NAS 3-2595 and by the Aerospace Research Laboratories, Office of Aerospace Research, under Contract AF 33-(657)-7380. The article is reproduced herein with the permission of the American Welding Society.
} 
and to the wall (in addition to radiation and bulk convection) by thermal conduction and by enthalpy convection caused by the interdiffusion of atoms and molecules. The ratio of the intensities of the last two mentioned transfer processes is determined by the magnitude of a dimensionless parameter called the Lewis number (see Heat Flow, Appendix A, page 91, Ref.).

$$
L e=\frac{\rho D c_{p f}}{k_{f}}
$$

which may here be defined with the frozen specific heat $\left(c_{p f}\right)$ at constant pressure and the frozen thermal conductivity $\left(k_{f}\right) ; \rho$ denotes the density and $D$ the diffusion coefficient considering the gas as a two component mixture of atoms and molecules. The following relation describes the overall heat flux $\left(\dot{q}_{w}\right)$ into the skin surface per unit area and time (excluding radiation):

$$
\dot{q}_{w}=h\left(i_{e}-i_{w}\right)
$$

The symbol $h$ denotes the heat transfer coefficient; $i_{e}$ and $i_{w}$ are the total enthalpies at the other edge of the boundary layer and at the skin surface respectively. These values have to include the energy of dissociation. A Nusselt number $\mathrm{Nu}$ is defined as:

$$
N u=\frac{h L c_{p f}}{k_{f}}
$$

with $L$ indicating a characteristic length. For laminar boundary layer flow of a gas with a Lewis number equal to one, the relation

$$
\mathrm{Nu}=\mathrm{CPr}_{\mathrm{f}}^{1 / 3} \sqrt{\mathrm{Re}}
$$


presents a good approximation to the results calculated by Lees, ${ }^{96}$ Fay and Ridde11,97 and others. The dimensionless parameters appearing on the right-hand side of the equation are the Prandtl number, a dimensionless transport property of the fluid, here again defined with frozen properties:

$$
P r_{f}=\frac{\mu C_{p f}}{k_{f}}
$$

and the Reynolds number:

$$
\operatorname{Re}=\frac{\rho V L}{\mu}
$$

$\mu$ is the viscosity and $V$ is the gas velocity outside the boundary layer. The constant $C$ in Equation 11 has the numerical value 0.57 for two dimensional stagnation flow and 0.76 for a rotationally symmetric stagnation point. The properties appearing in the above equations vary throughout the boundary layer as a consequence of temperature variation and properly defined mean values have to be introduced. 98 In a gas which is dissociated but not ionized, the Prandt number assumes values between 0.6 and 1 . The Lewis number has values between 1 and 2 . Corrections have been established by which Equation 11 can be adjusted to a value of the Lewis number somewhat different from unity. 97

If the temperature is sufficiently high for thermal ionization, electrons and ions appear in addition to neutral particles in the gas. The Prandtl number is now strongly reduced because of electron conduction and may drop at sufficiently high temperature to values as low as 0.01 which are comparable to those of metals.

Figure 48 presents the frozen Prandt 1 number of argon according to calculations by Penski ${ }^{99}$ and Park. 100 The equilibrium Prandt 1 number is also plotted. It increases at high temperatures caused by the enthalpy of interdiffusion. One might assume that the heat flux is increased drastically in such a situation. This, however, is not the case on a cold 


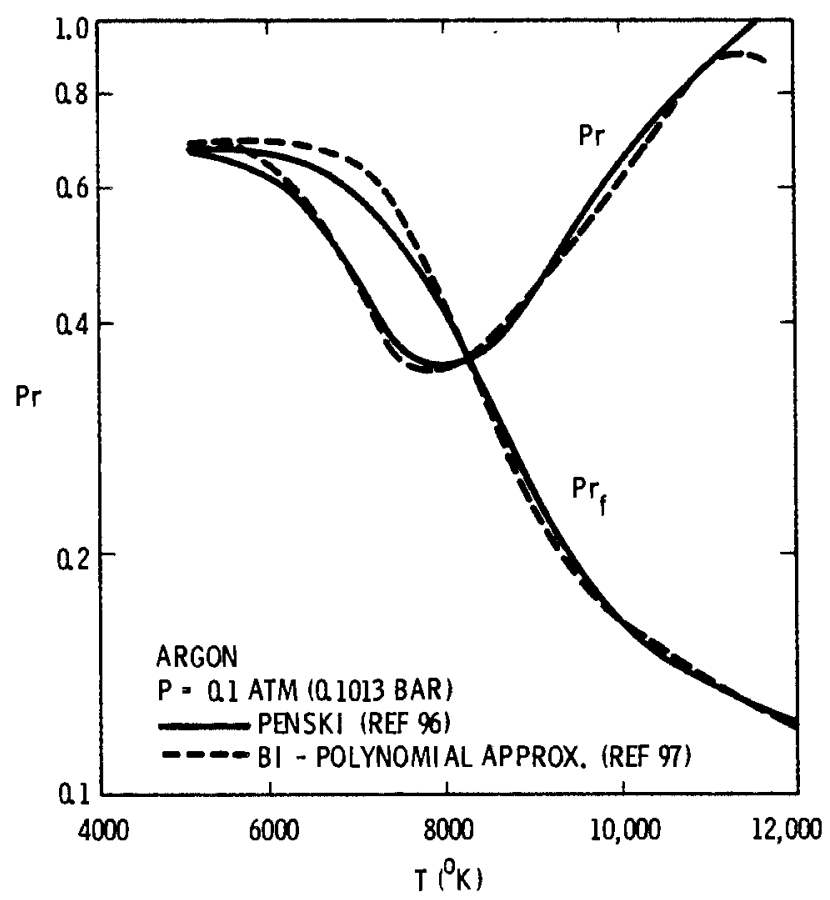

FIGURE 48. PRANDTL Number for Frozen and Equilibrium Composition 100

catalytic wall because the surface will always be separated by a relatively cold non-ionized layer from the hotter part of the boundary layer. This layer will be relatively thick when chemical equilibrium between charged and neutral particles is established within the boundary layer. It will be somewhat thinner for a chemically frozen boundary layer. Only a noncatalytic surface can establish an electron density with finite values through the whole range of the boundary layer, and electronic conduction can then cause the heat transfer to the skin to increase by an order of magnitude.

There still exists another process which tends to reduce the heat transfer. Interdiffusion of electrically charged and uncharged particles is determined by ambipolar diffusion. The diffusion coefficient $\left(D_{a}\right)$ for this process is assumed to be approximately twice as large as the diffusion coefficient for interdiffusion of neutral particles. It, therefore, maintains in an ionized gas its order of magnitude in the Lewis number whereas 
the thermal conductivity $\left(k_{f}\right)$ increases by at least one order of magnitude. This causes the Lewis number to assume smaller values than 1 , and correspondingly the correction which has to be applied to Equation 11 reduces the Nusselt number. This is so because Equation 9, which connects the energy flux $\left(q_{w}\right)$ with the total enthalpy difference, over-estimates the interdiffusion process for a Lewis number smaller than 1 .

The considerations which have been presented above are borne out by boundary layer analyses and experiments. Figure $49^{100}$ taken from Park 100 summarizes such results. The enthalpy $\left(i_{e}\right)$ is used on the abscissa of Figure $52^{101}$ because the enthalpy $\left(i_{W}\right)$ corresponding to the skin surface temperature is negligible compared with $\left(i_{e}\right)$. The properties on which the heat transfer parameter $\mathrm{Nu} / \sqrt{\mathrm{Re}}$ appearing on the ordinate is based have been introduced at the skin surface temperature and are, therefore, for the non-ionized gas.

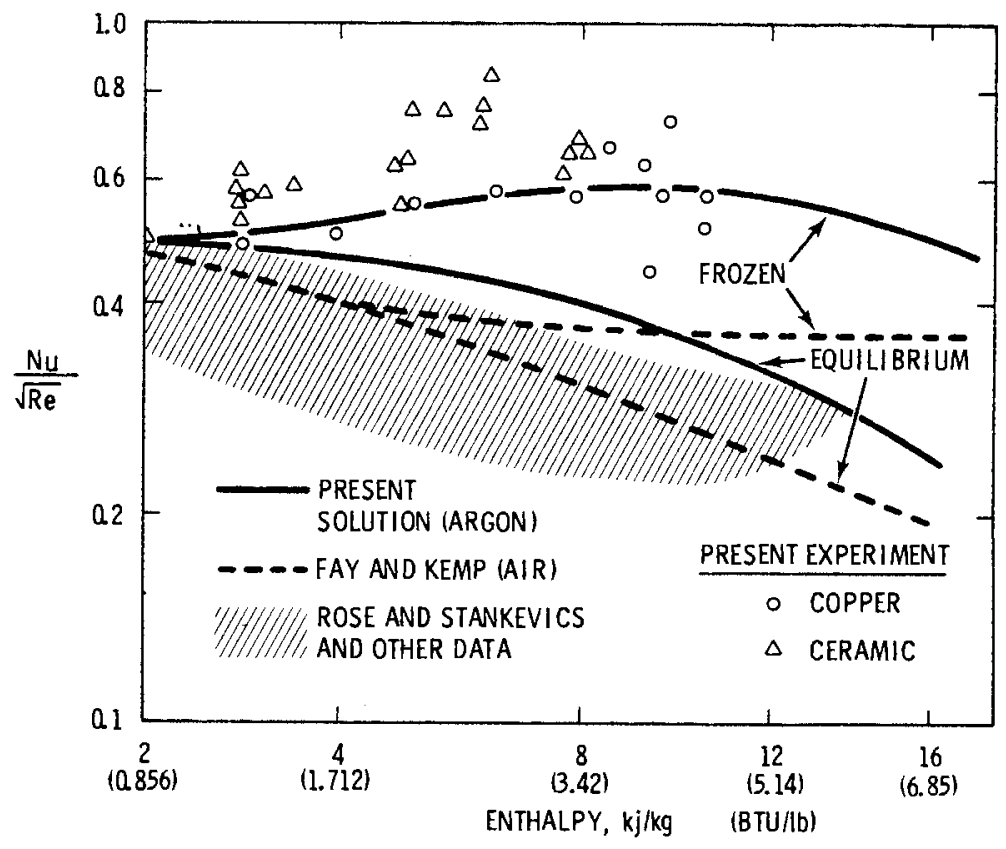

FIGURE 49. Heat Transfer Parameter Determined Analytically and Experimentaliy as a Function of Gas Enthalpy 100 


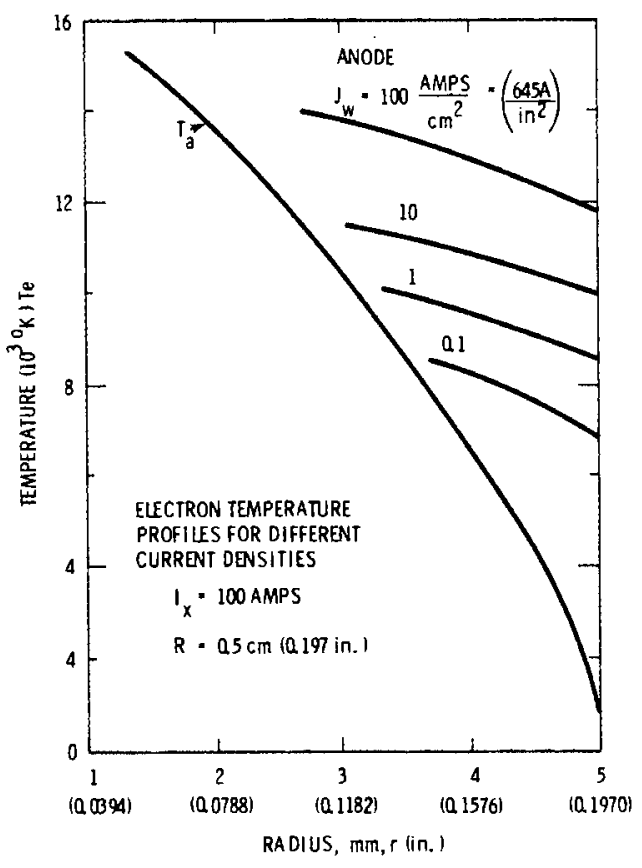

FIGURE 50. Profiles of Gas Temperature $T_{a}$ and Electron Temperature $T_{e}$ in a Wa 11 Stabilized Arc ( $I_{x}$ Ax $\left.i d\right]$ Current, $J_{W}$ Current Density to Biased Wall Segment) 101

It will be observed that the parameter $\mathrm{Nu} / \sqrt{\mathrm{Re}}$ maintains, in general, its order of magnitude regardless of the value of the gas enthalpy. Equilibrium boundary layers exhibit somewhat lower values of the heat transfer parameter than frozen boundary layers in agreement with the considerations above. The experiments appear to indicate that the boundary layers were chemically frozen. In summary then, heat transfer can be calculated with the equations above with some degree of accuracy. The main uncertainties are caused by insufficient knowledge of transport properties at high temperatures.

The skin exposed to an ionized gas flow assumes a negative electric potential relative to the plasma when it is an electric conductor. This is a consequence of the large thermal velocities of the electrons. A positive space charge is established simultaneously in a thin sheath of gas bordering the skin surface. The thickness of the sheet is generally an order of magnitude smaller than the boundary layer thickness. 


\section{Plasma Heat Transfer With Current Flow to an Anode 95}

The interaction of the various processes contributing to the energy transfer to a surface changes considerably when an electric current flows into the surface. The following discussion deals with this situation which is frequently found in high intensity arcs. Especially interesting is the anode regime of such arcs which provides a flexible and to a certain degree also controllable current attachment region. Even with the simplest arc geometries operated in inert gas atmospheres, however, secondary effects (e.g. anode and cathode plasma jets or arc fluctuations) are inevitable, they frequently disclose a very severe influence on the anode energy transfer. This fact requires a careful consideration of these secondary effects.

The main features of the energy transfer process can be described by a model which considers the plasma bordering the anode surface as a mixture of two gases: an electron gas and a heavy gas consisting of ions and neutral particles. The electrons are now sucked into the anode; as a consequence, their time of residency in the boundary layer and in the sheath (which assumes now a negative space charge) is so short that the electrons are not able to equalize their thermal energy with the heavy particles. It appears that one can approximate the condition created in this way by specifying an electron temperature which is now different from the temperature of heavy particles.

This situation is well demonstrated by the results of an analysis presented in Figure 50. 101 The analysis deals with a wal1-stabilized arc. It should, however, apply qualitatively to a boundary layer as well. Figure 50 indicates that the electron temperature stays almost constant throughout the boundary layer and the sheath region at higher current densities drawn to the surface. The fact that high energy electrons are sucked into the anode is the main reason for the large increase in heat flux since it occurred on a surface through which an electron current flows rather than a surface with zero current. 


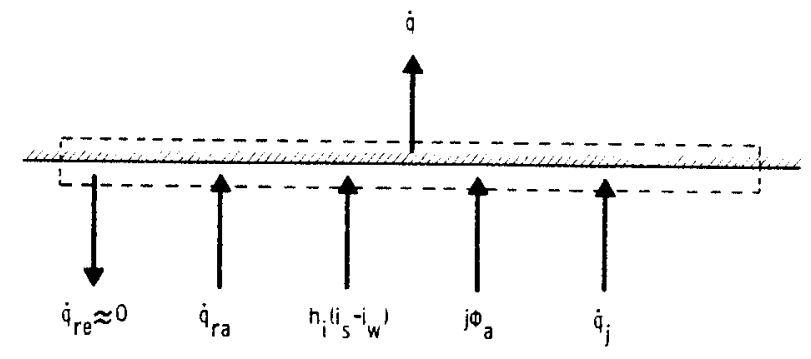

FIGURE 51. Energy Balance of Anode Surface Element ${ }^{95}$

One of two problems arise in the development of engineering devices utilizing electric arcs: the total heat flux to a water-cooled current carrying surface represents in many applications the predominant energy loss which diminishes the efficiency of the respective plasma device. Therefore, efforts have been made to reduce such losses. Transpiration cooling has been considered because part of the heat flux to a surface is by this method recovered by the gas transpiring through the surface. 102,103

The second problem is connected with the specific heat flux to a current carrying surface; this may become so high that local melting occurs despite application of the most efficient water cooling system which is able to remove about $10 \mathrm{~kW} / \mathrm{cm}^{2}\left(64.5 \mathrm{~kW} / \mathrm{in} .^{2}\right)$. This situation has been experienced in high pressure arcs, even at moderate currents using working fluids with high heat conductivity (for example hydrogen). The high pressure as well as the high heat conductivity of the working fluid leads to a constricted anode attachment resulting frequently in anode failure. The 
properties of the anode sheath and of the boundary layer apparently have a decisive influence on the current transition and, therefore, also on the local heat transfer to the anode.

A cold surface favors a constricted attachment in order to keep the electron temperature and, with it, the electric conductivity sufficiently high despite the intense cooling of the boundary layer. High temperature surfaces may permit a more diffuse current transition. To prevent too high specific heat loads at the surface the anode arc terminus may be moved with high velocities over this surface distributing the heat flux to a larger area. Heated anodes offer another possibility to keep specific heat fluxes within reasonable limits.

In the following sections anode heat transfer studies will be reviewed. These have been carried out on various arc geometries and under various test conditions. For many of these investigations energy transfer models have been proposed in order to determine the contribution of the different energy transfer modes to the total anode heat flux. Detailed anode heat transfer studies are available for arcs burning in argon and for water-cooled copper anodes which permit rather accurate over-a11 and local heat transfer measurements. Therefore, this review will only deal with results obtained from such anodes.

\section{Free Burning Arcs With Plane Anodes}

The characteristic arc geometry of the free-burning, high-intensity arc shows a well-known bell-shaped stationary arc column. 104,105 The reason for the steadiness of this arc column is the strong cathode jet generated by magnetohydrodynamic forces in the cathode region. 106,107,108 This region acts as an electromagnetic pump drawing gas from the surroundings and ejecting it toward the anode in the form of a jet. The cathode has an important influence on the convective part of the anode heat flux, especially at small arc lengths and at high currents.

The energy transfer to the anode involves a variety of transfer mechanisms. These can be described by an energy transfer model $102,109,110,111$ based on the observations at the start of this section and indicated in the 
scheme of Figure $51 .{ }^{95}$ The energy transferred to the anode consists of:

- Thermal and kinetic energy of the electrons comprising the arc current and penetrating the anode surface:

$$
\dot{q}_{j}=\frac{j}{e}\left(5 / 2 k T e+e U_{a}\right)
$$

where $\dot{q}_{j}$ is the energy flux per unit area connected with current flow into the surface, $j$ is the current density, e the electronic charge, $k$ is the Boltzman constant, $T_{e}$ is the electron temperature, and $U_{a}$ is the anode fall of potential.

$$
\dot{\mathrm{q}} \Phi_{\mathrm{a}}=\jmath \Phi \mathrm{a}
$$

- Convective heat transfer from the hot plasma through the boundary layer $\dot{q}_{\text {conv }}$.

- Radiative heat transfer from the arc plasma: $\dot{q}_{r a}$.

Electrons suffer elastic collisions with heavy particles in the boundary layer and the anode sheath. This is a process that will reduce the part of the electron current stagnation enthalpy which is transferred to the anode. However, the number of elastic collisions in the thin fall region is not large enough to transfer an appreciable amount of energy to the heavy particles since the energy transfer per collision is very sma11. The energy loss of the electrons to heavy particles will therefore be essentially only the amount required for ion generation in this region. The ion current density in turn is only of the order of 1 percent of the total current density so that the energy loss of the electrons traveling through the anode fall space may be neglected.

The energy carried away from the anode surface consists of:

- The heat $\dot{q}$ conducted away from the anode surface by the cooling water, and

- The heat $\dot{q}_{\text {re }}$ radiated away from the anode surface to the environment. 


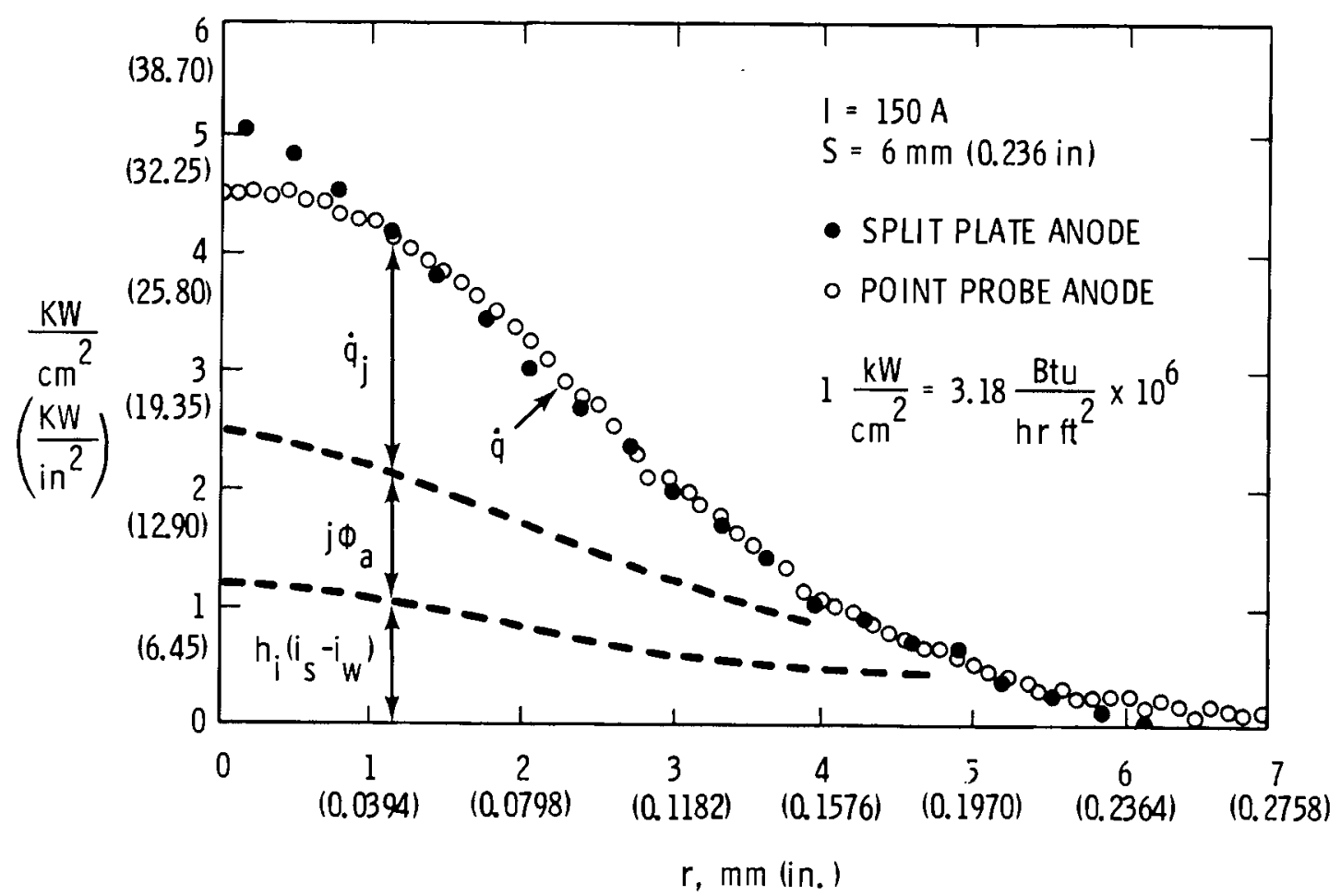

FIGURE 52. Radial Distribution of Energy Transfer at Anode Surface 95

Under steady state conditions the energy balance for a surface element of the anode can be written as:

$$
\dot{q}+\dot{q}_{r e}+\dot{q}_{a b l}=\dot{q}_{j}+\dot{q}_{a}+\dot{q}_{r a}+\dot{q}_{c o n v}
$$

In normal operation the anode surface is kept well below the melting point, therefore, $\dot{q}_{r e}$ and $\dot{q}_{a b l}$ are negligible. All other quantities besides $U_{a}$ and $\Phi_{a}$ can be experimentally determined. The results of such measurements in a current range from 50-150 A 102,109,110,111 indicate that the heat flux due to the electron flow to the anode is the predominant heat transfer mechanism. A typical diagram of such measurements is shown in Figure 52.101 The convective heat transfer was calculated with Equation 9 assuming that this equation holds for a current carrying surface. Measurements by Nestor 112 for currents up to 300 A show essentially the same results. Overa11 energy balances demonstrate ${ }^{113}$ that up to 85 percent of the arc power 
input is transferred to the anode (with electrode gaps up to $20 \mathrm{~mm}$ [26/32 in.]) and the percentage of energy transfer to the anode is about independent of the current.

Another interesting finding of these studies is the behavior of the current density which maintains its maximum value at the anode center regardless of the total current $(100 \mathrm{~A}<\mathrm{I}<400 \mathrm{~A})$. The $\operatorname{arc}$ column and especially the anode attachment region spreads correspondingly out with increasing current, and it appears that the arc adjusts its attachment area to that current density which provides a sufficiently high electron temperature and electric conductivity, Figure 50. The corresponding anode heat fluxes reported by Schoek $102,109,110,111$ are in the range of $4-6 \mathrm{~kW} / \mathrm{cm}^{2}(25.8$ $38.7 \mathrm{~kW} / \mathrm{in}^{2}$ ).

The convective heat flux density increases with increasing current, corresponding to nearly a linear increase of the velocity with which the cathode jet approaches the anode. This velocity reaches a value of $180 \mathrm{~mm} /$ $\sec (509 \mathrm{ft} / \mathrm{sec})$ at $150 \mathrm{~A}$ and $\mathrm{a} 6 \mathrm{~mm}(0.236 \mathrm{in.})$ cathode-to-anode distance.

In a more recent thesis by Eberhart 114 the current range has been extended to $1100 \mathrm{~A}$. The percentage of the energy transferred to the anode up to $1100 \mathrm{~A}$ is again independent of the current. However, the electron heating of the anode which predominates at lower currents is surpassed by gaseous convection at very high currents depending upon the electrode gap. This fact is, at least in part, a consequence of another finding which indicates that the local current density at the stagnation point even decreases with increasing current.

\section{Cathode Axis Parallel to a Plane Anode}

When the cathode is orientated parallel to the anode surface, one obtains quite a different shape of the plasma column as shown in Figure 53 . The cathode jet now impinges against an anode jet which is generated by a current density gradient toward, and in the immediate vicinity of, the anode. The reason for the fact that the arc column tends to attach to the anode in a rather contracted manner is probably due to a low temperature gas layer on the anode surface. This constriction is usually not as strong 

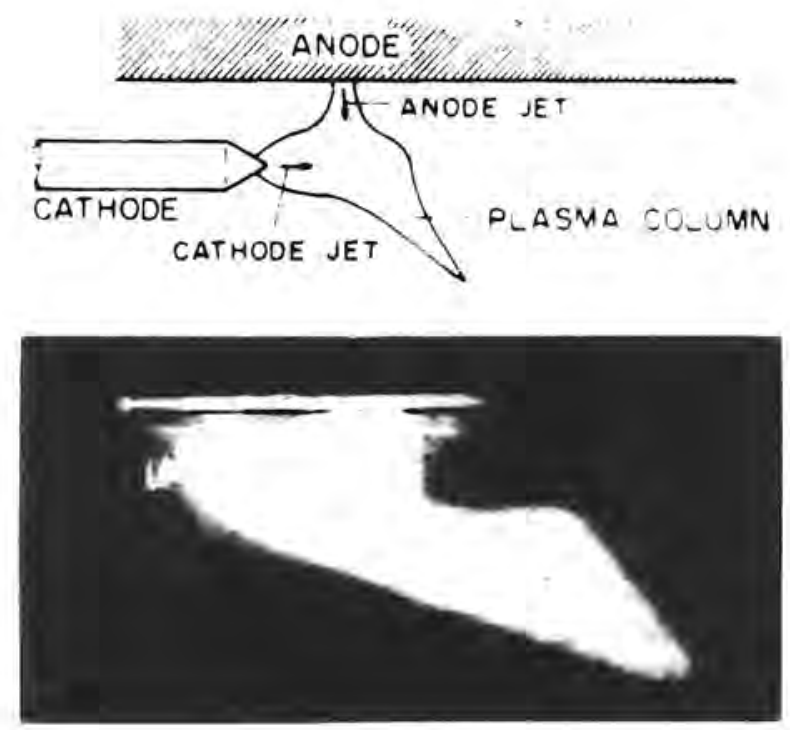

FIGURE 53. Impinging Jets. A (Top) Schematic; B (Bottom) Photograph95

as in the cathode region. 115 The heat flux per unit area is expected to have a distribution curve simiiar to the one shown in Figure $50^{101}$ but with a smaller attachment area and a correspondingty larger maximum. From the photographs it appears that the maximum may be 100 times as high. The local heat flux at the anode will, under such conditions, surpass the highest permissible value and it is, therefore, fortunate that a superimposed axial gas flow causes the arc frequently to fluctuate leading to a moving anode arc terminus. This spreads the anode heat flux over a wider surface area and reduces the maximum temperature of the anode appreciably.

An estimate of the reduction in the maximum temperature which is obtained in this way can be found from the following model: Consider a semi-finite solid with a point heat source moving with constant velocity $v$ along a straight path on its surface. The temperature field created in this way in the solid material is steady when viewed by the observer who 
moves along with the heat source. Surfaces of constant temperature have a shape similar to half an elipsoid and are described by the following equation: 116

$$
\begin{aligned}
& v=\frac{1}{2 \pi P e_{r}} \exp \left[-\frac{P e_{r}}{2}\left(\frac{P e_{x}}{P e_{r}}+1\right)\right] \\
& v=\frac{(T-T \infty) k^{2}}{\rho c_{p} v \dot{q}}, P e_{r}=\frac{v r}{\alpha}, P e_{x}=\frac{v x}{\alpha}
\end{aligned}
$$

in which $v$ denotes a temperature parameter, and Pe denotes a dimensionless parameter which is frequently used in heat transfer analysis and is called the Peclet number. $T$ is the local temperature in the solid at the radial distance $r$ from the heat source, $T_{\infty}$ is the temperature at $r=\infty$, $k$ the thermal conductivity, $p$ is the density, and $c_{p}$ is the specific heat of the solid. $\dot{q}$ is the heat flux released by the point source per unit time, $x$ the projection of $r$ onto a straight line through the heat source in the direction of its movement, and $\alpha$ is the thermal diffusivity of the material.

The curves in Figure 54 present the intersections of the isotherma 1 surfaces with the plane surface of the solid. Equation 17 can also be interpreted as describing the temperature of the material when instead of the point source a situation is considered in which the heat flux $\dot{q}$ is distributed over any of the isothermal surfaces. On this basis a comparison can be made with the corresponding temperature field of a stationary heat source (described in more detail in the Heat Flow Appendix IV-B-5-a-ii) with the result that the ratio $\mathrm{v} / \mathrm{v}_{\mathrm{s}}$ of the temperature parameter of the moving heat source to the parameter $v_{s}$ of the stationary heat source depends on the Peclet number $\left(\mathrm{Pe}_{r}\right)$ in a way as listed in Table 3 . It can therefore be seen that the maximum temperature increase in the solid material is reduced to about one-third of the value createu by a stationary heat source of equal strength when the Peclet Number $P e_{r}$ has a value of 10 . 


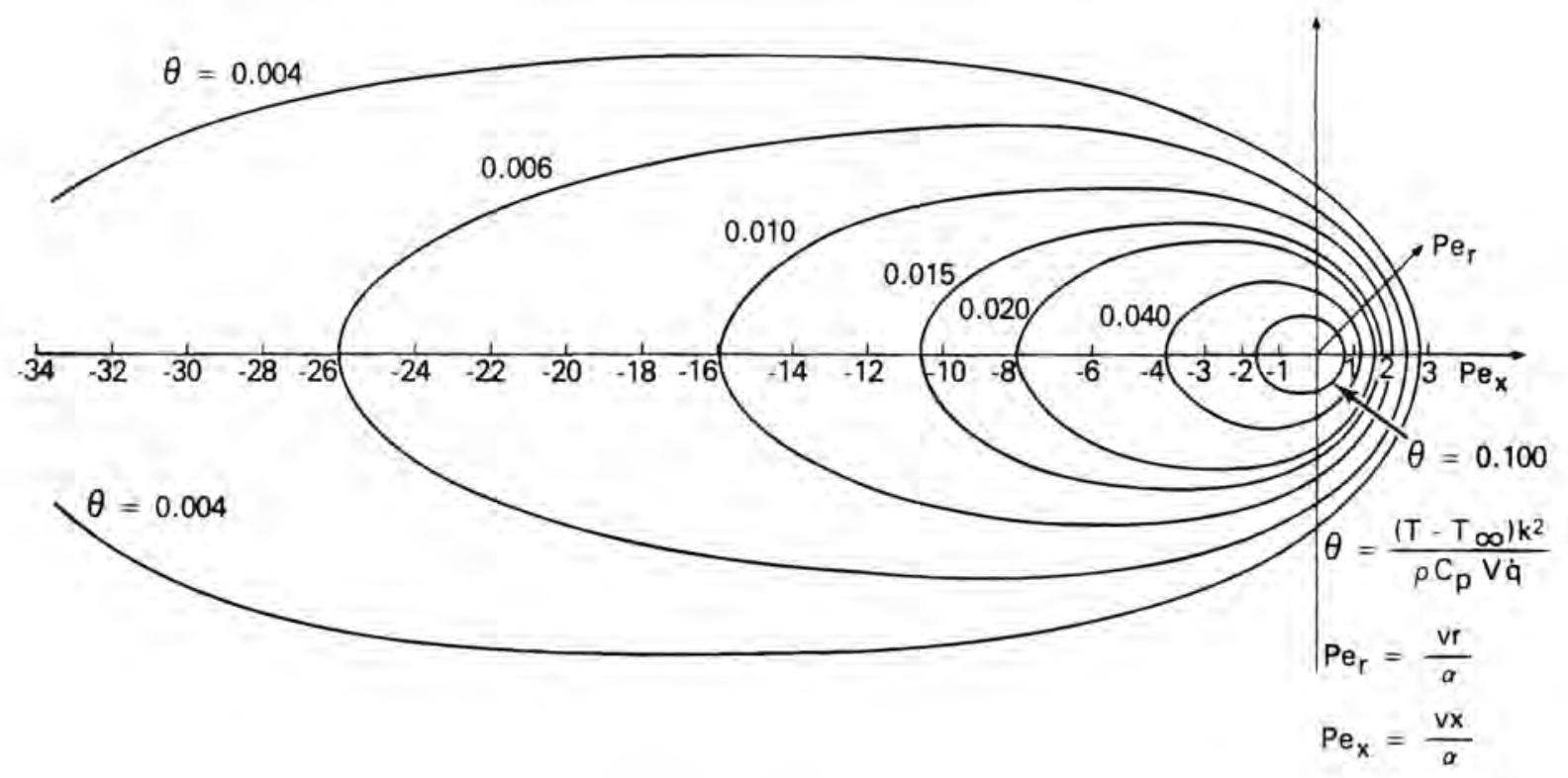

FIGURE 54. Temperature Field Created by a Moving Point Heat Source On the Surface of a Semi-Infinite Body 95

TABLE 3. Dependency of Temperature Parameter Ratio On the Dimensionless Parameter $\mathrm{Pe}_{r} 119$

$\begin{array}{ccccccc}\mathrm{Pe}_{r} & 1.1 & 2.25 & 3.9 & 4.82 & 6.7 & 10 \\ \nu / \nu_{s} & 0.69 & 0.56 & 0.49 & 0.45 & 0.42 & 0.39\end{array}$


The fluctuating arc repeats its movement down the anode almost periodically and the maximum temperature in the solid material will be somewhat larger than the value obtained by the estimate described above. The model suggested here can certainly be refined, taking into account the fact that the anode usually is a thin wall cooled on the backside by water and considering a heat flux as presented in Figure $54 .^{95}$ It is felt, however, that the model already describes the order of magnitude correctly in its present form.

No measurements of the distribution curve of the heat flux or of the temperature distribution in the anode have been performed up to now with which the above analysis can be compared. Some indication of the relief provided by the arc movement is obtained by the results of some tests in which the anode was composed of segments with a width of $1.25 \mathrm{~cm}(0.492 \mathrm{in}$.) measured in flow direction. The results are presented in Figure $55 \mathrm{~A}^{95}$ and $55 \mathrm{~B}^{95}$ in which $\dot{Q}_{S}$ represents the flux into an individual segment and $\dot{Q}_{A}$ the total anode heat flux.

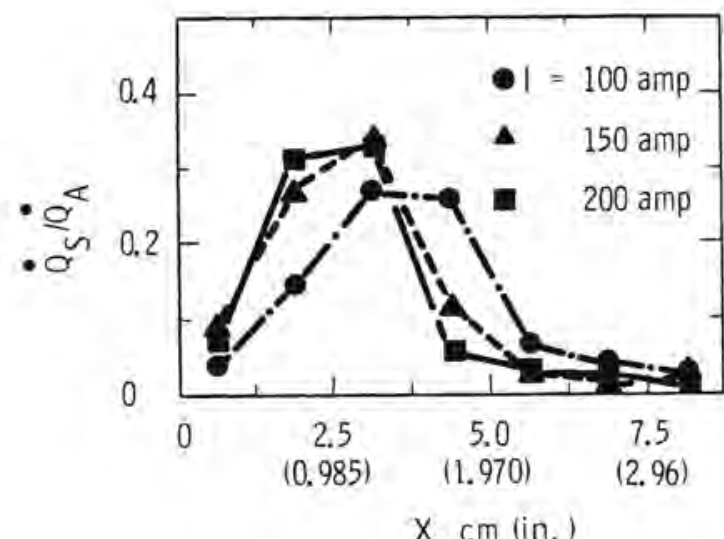

$x, \mathrm{~cm}$ (in.)

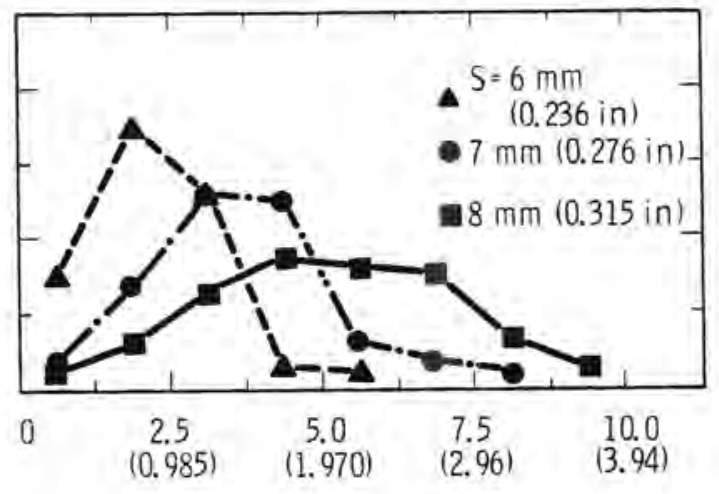

$x, \mathrm{~cm}$ (in.)

FIGURE 55A \& B. Distribution of Energy Transfer at Anode Surface, Argon, $P=380 \mathrm{~mm} \mathrm{Hg}(15 \mathrm{in}$. $\mathrm{Hg}), v=100 \mathrm{~m} / \mathrm{sec}$. $(328 \mathrm{ft} / \mathrm{sec}))^{95}$ 
Figure $56^{95}$ shows the time dependence of the current flux to the segments with different parameter settings. The six traces on the oscillograms, starting from the top, show the individual currents through four consecutive segments and the tota 1 current as a d-c signal and the overall voltage as an a-c signa1. In the third oscillogram, under $380 \mathrm{~mm}$ (14.96 in.) Hg pressure column, of the second row a complete cycle of the so-called "fluctuating arc" is clearly demonstrated. The first cycle shows a re-striking of the arc at the first segment, transition to segment two, three, and four, and decay of the arc at segment four accompanied by re-striking again at the first segment. The following cycles show essentially the same behavior.

In a sequence of frames taken from a high speed movie, Figure 57 , the actual anode attachment and its movement is shown in a complete cycle, frame 2 through 12. Analys is as we11 as experiments indicate the maximum temperature in the anode can be considerably reduced by the movement of the arc, and it is therefore of interest to know under which conditions that the arc fluctuates and under which conditions it does not.

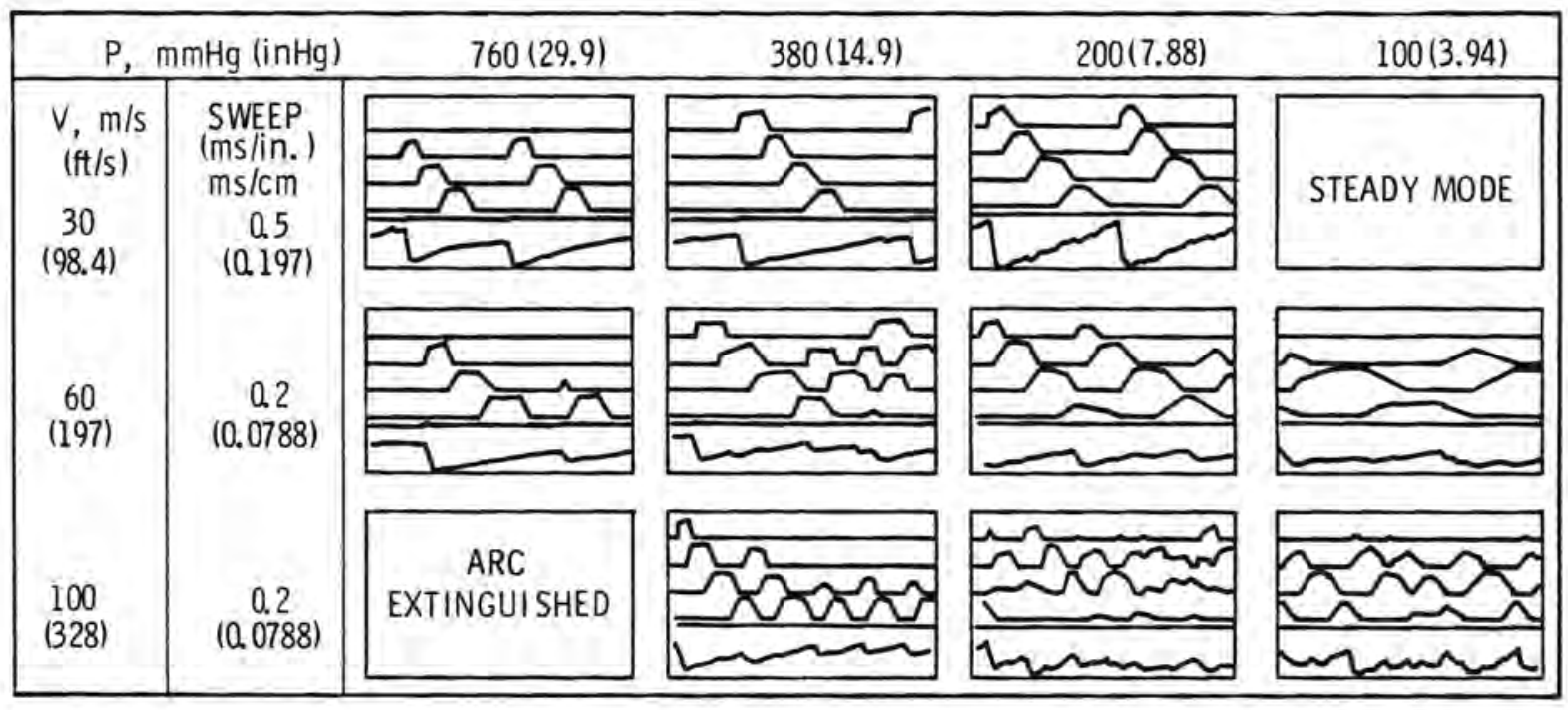

FIGURE 56. Time Dependence of the Current Distribution for the Segmented Anode 95

(Argon, $I=100$ Amp., $S=9 m m$ (0.354 in.) 

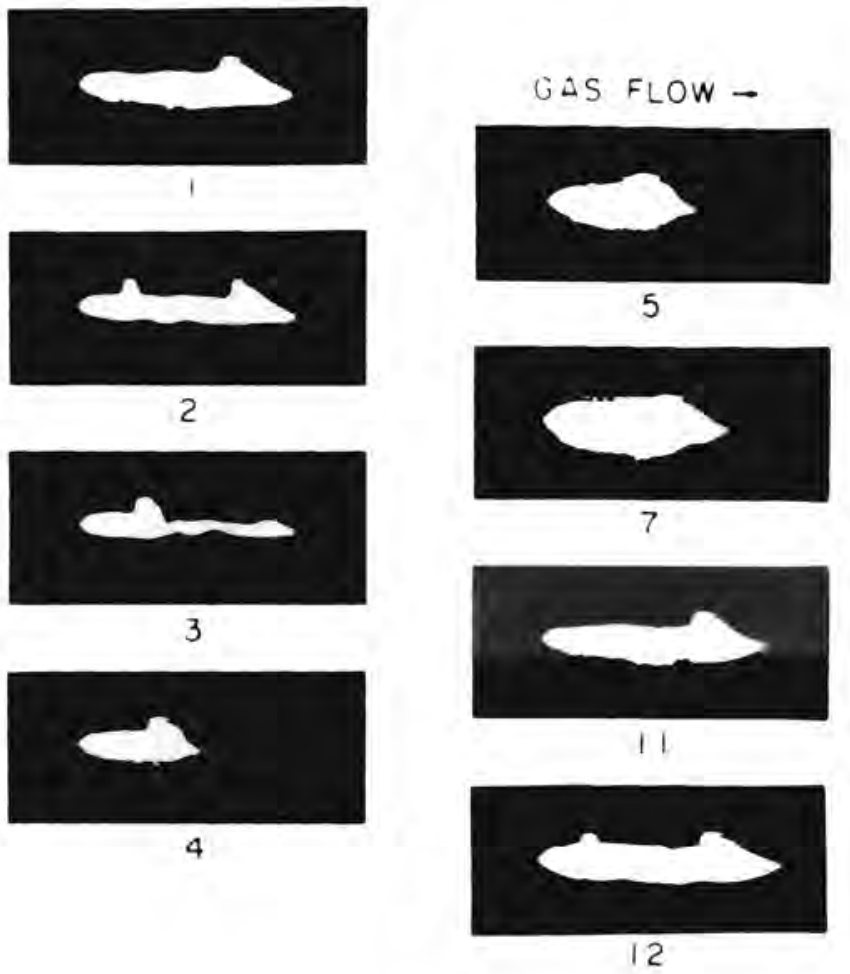

FIGURE 57. Frames From High Speed Movies Demonstrating Fluctuating Mode in Argon95 
Figure $58^{95}$ presents the results of pertinent experiments. The curves in this figure separate the domains of the two modes of arc operation. The figure indicates that the magnitude of electric current shifts the curve separating the two modes only to a minor degree; this leads to the conclusion that the flow and the energy transfer process are dominant in determining the mode of operation. It is reasonable, however, to assume that a temperature field for a line heat source similar to the one in Figure $54^{95}$ describes the conditions in the neighborhood of the arc in that part of the column which is normal to the flow direction. It will of course, be modified by the large density variations.

The Peclet number $\mathrm{Pe}$ is:

$$
P e=\frac{v r}{\alpha}=\frac{\rho c_{p} v r}{k}
$$

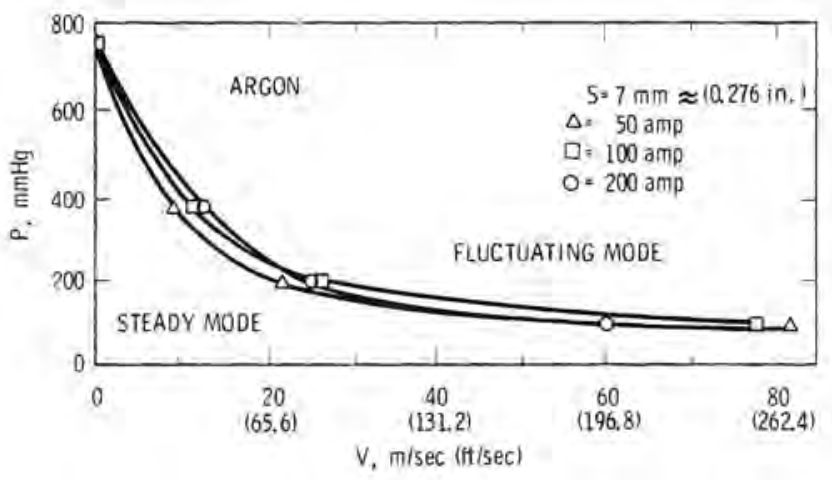

FIGURE 58. Steady and Fluctuating Mode of an Arc (Parameter: Arc Current) 95 
This indicates that the ratio of the convective transfer process determines the temperature field. A check on the curves in Figure $58^{95}$ shows that they are quite wel1 represented by hyperbolas, pv - constant, which for approximately constant temperature leads to $\rho v=$ constant. This suggests the conclusion that a characteristic Peclet number may be the main parameter determining the arc mode. On the other hand, viscous effects may also be of influence. This is so since the attachment of the arc occurs through a boundary layer suggesting the influence of the Reynolds number. Further experiments with different gases may shed some light on these questions.

The fluctuating mode has also been achieved in engineering arc plasma devices by magnetically rotating the anode arc terminus or by using segmented anodes where each segment has been connected over a smal1 resistor to the power source. This forces the anode arc terminus either to rotate or to split into a number of anode arc attachment spots corresponding to the number of segments.

A comparison of the overall energy balance results obtained from this arc with those obtained from the classical free-burning arc shows a drastic reduction in the anode losses. In the latter case, between 75 to 85 percent of the electrical input energy was consumed by the anode; while in the present case, only 20 to 60 percent of the input energy is transferred to the anode. There are essentially two reasons for the drastic change in anode losses--the changing of the electrode configuration and the adding of a gas flow.

In the present case, the cathode jet is oriented parallel to the anode surface which results in a longer arc column and, therefore, also a high power input at a constant current reducing the fraction of the total energy which is transferred to the anode. At the same time, the convective part of the anode energy transfer caused by the cathode jet is almost eliminated. Experiments demonstrated, however, that the main reduction occurs when a gas flow parallel to the anode is added, causing a large amount of the heat generated in the arc to be carried away by bulk convection thus reducing the anode energy flux considerably. 


\section{Cylinder Geometry With an Annular Anode}

The wall stabilized cascaded arc arrangement ${ }^{117}$ exhibits well-defined arc parameters. This arrangement also represents a close approach to the arc flow, current transition and heat transfer situation found in actual arc plasma generators which are used in numerous applications. On the other hand, it offers fewer possibilities for detailed local studies than the geometries discussed before, and experiments are mainly confined to overal1 mean values.

A schematic diagram showing the type of apparatus used in these experiments is presented in Figure $59.9^{95}$ The arc is operated with a relatively high axial flow from the cathode end of the constrictor tube. The flow and the current transition are similar to that described in the preceeding section. However, the arc column is more constricted in the prevailing case; this leads to higher temperatures in the plasma column. Corresponding to the small length of the anode, the longitudinal movement of the anode arc

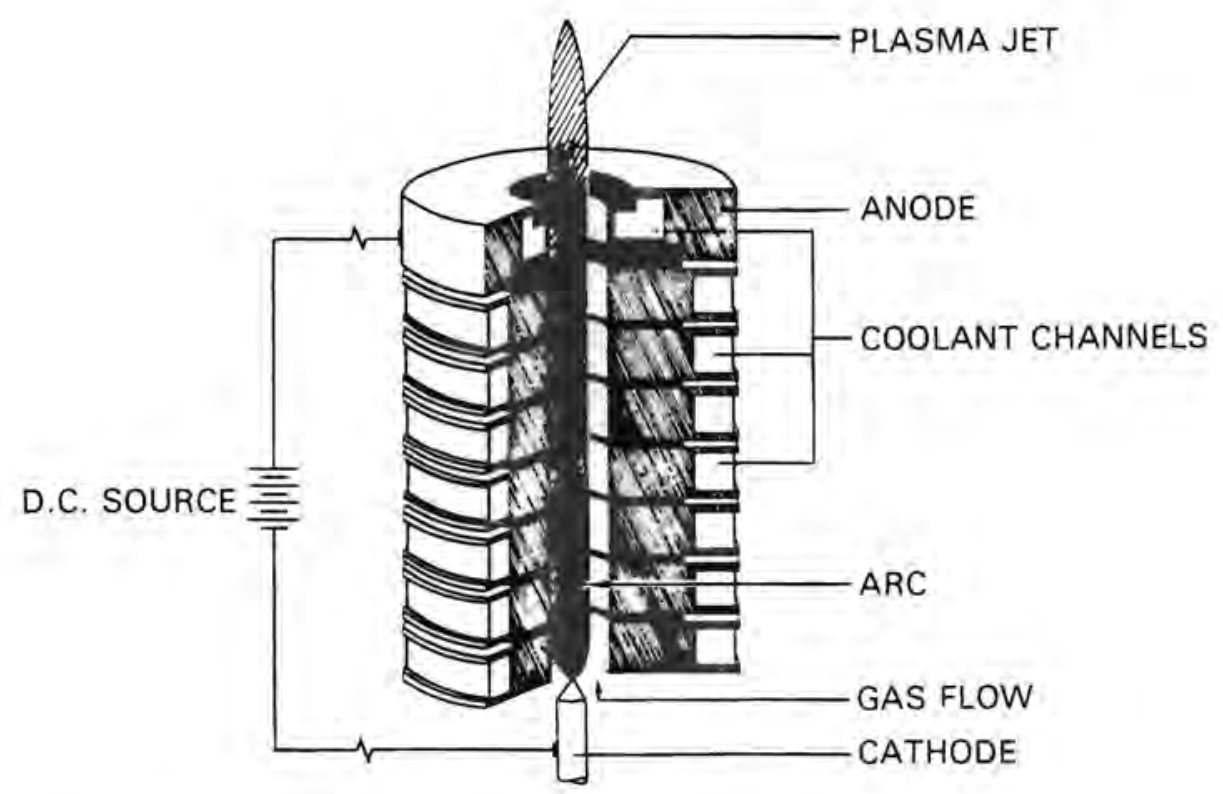

FIGURE 59. Schematic Drawing of Water-Cooled Cascaded Arc, Diagonal Hatching - Conducting Material, Solid Black-Electrical Insulator 95 
terminus is limited. Nevertheless, the same characteristic behavior of the arc vottage which is symptomatic for the fluctuating mode of the arc, and which has been clearly demonstrated with plane anode, $5,118,119$ may also be found in cylindrical geometries. 120,121 Therefore, the basic heat transfer situation at the anode will remain the same.

In order to distinguish between the various energy transfer modes (especially between the contribution of the current flux and the other heat transfer mechanisms) an anode energy transfer model is established, but in this case, for the overall energy fluxes. The single energy transfer modes are the same as discussed previously. Combining the convective and radiative energy transfer into one term, the overall energy balance may be written as:

$$
\dot{Q}+\dot{Q}_{r e}+\dot{Q}_{a b 1}=\dot{Q}_{I}+\dot{Q}_{\Phi a}+\dot{Q}_{r a}+c
$$

where the designation of the terms is the same as in Equation 16 with one exception of the last term $\dot{Q}_{r a}+c$ which is:

$$
\dot{Q}_{r a}+c=\dot{Q}_{r a}+\dot{Q}_{c o n v}
$$

The terms $\dot{Q}_{a \mathrm{~L}}$ and $\dot{Q}_{r e}$ may be neglected because the anode surface temperature is kept well below the melting point. $\dot{Q}$ is determined calorimetrically, and $\dot{Q}_{\Phi a}$ can be calculated from the anode work function. The values of work function of metals found in the tables refer usually to vacuum conditions. The surface conditions which are doubtless quite different at the anode of an arc discharge may cause a drastic change of the work function. Therefore $\dot{Q}_{\phi a}$ may be appreciably in error by using these values for the work function.

The term $\dot{Q}_{r a+c}$ is determined in the following way: Over the length of the constrictor tube where the flow is usually developed, the heat transfer per unit area should be constant. Therefore, $\dot{Q}_{r a+c}$ can be determined from 
the heat flux to a segment situated in the thermally developed region, because the only heat flux to such a constrictor segment is the convective and radiative transfer from the plasma. The typical diagrams for two different flow rates $\dot{m}_{C}$ through the constrictor tube are shown in Figures $60 A$ and $B$. From this, Figures $60 A$ and $B$, the energy acquired by the electrons in the anode fall region is seen to be the most important single factor in determining the heat transfer to the anode. Convection and radiation are more than an order of magnitude smaller than the total contribution of the current to the anode energy flux. More details about the experimental procedure may be found in the literature. 103

\section{A. Heat Flow Appendix}

i. Energy Flux Relationships. The energy flux $(\dot{q})$ per unit time and area in a two-component gas through a plane through which no bulk flow occurs is given by the following equation:

$$
\dot{q}=-k_{f} \frac{\partial T}{\partial n}-\rho D \frac{\partial w_{1}}{\partial n}
$$

where $k_{f}$ is the frozen conductivity, $\rho$ is the mixture density, $D$ is the mass diffusion coefficient, $w_{1}$ is the mass fraction of one component, and $n$ is the normal to the plane. The enthalpy ( $i$ ) of such a mixture is a function of temperature $(T)$ and of the mass fraction $\left(w_{1}\right)$. The derivative of the enthalpy in the direction $n$ can therefore be written as:

$$
\frac{\partial i}{\partial n}=\left(\frac{\partial i}{\partial T}\right)_{W_{1}} \frac{\partial T}{\partial n}+\left(\frac{\partial i}{\partial W_{1}}\right)_{T} \frac{\partial w_{1}}{\partial n}
$$

The term $(\partial i / \partial T)_{w_{1}}$ is the specific heat for a situation in which the composition is locally constant, the so-called frozen specific heat. The term $\left(\partial i / \partial w_{1}\right)$ is for an ideal gas mixture $\left(i_{1}-i_{2}\right)$. In this way Equation 22 can be transformed into:

$$
\frac{\partial i}{\partial n}=c_{p f} \frac{\partial T}{\partial n}+\left(i_{1}-i_{2}\right) \frac{\partial w 1}{\partial n}
$$




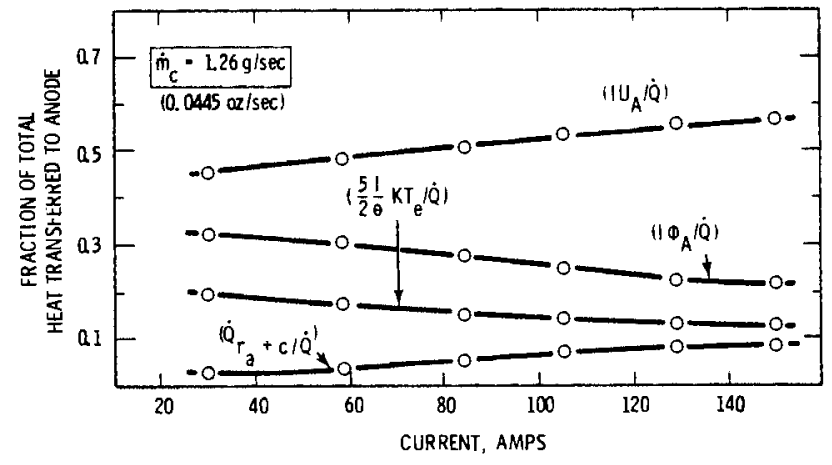

FIGURE 60A. Fraction of Total Heat Transferred to Anode Shown in Figure 59 by Indicated Transfer Mechanisms 95

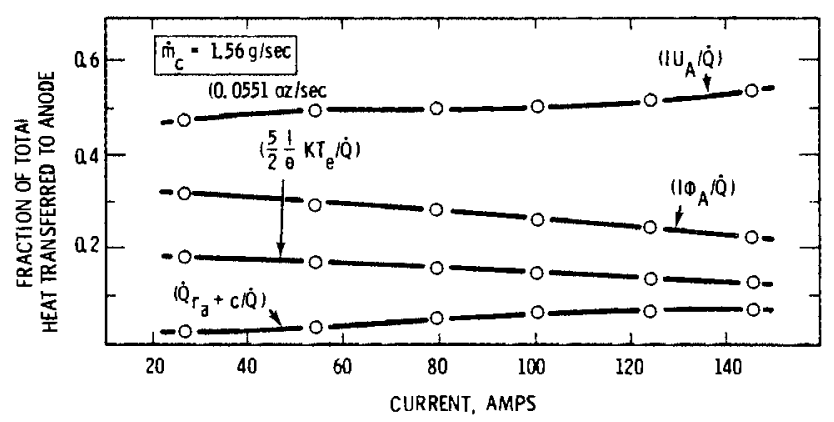

FIGURE 60B. Fraction of Total Heat Transferred to Anode Shown in Figure 59 by Indicated Transfer Mechanisms 95 
This equation is now used to replace the temperature derivative in Equation 20 with the result:

$$
\dot{q}=-\frac{k_{f}}{c_{p f}} \frac{\partial i}{\partial n}-\rho D\left(1-\frac{1}{L e}\right) \times\left(i_{1}-i_{2}\right) \frac{\partial w_{1}}{\partial n}
$$

with the Lewis number Le given by Equation 7. For a gas mixture with a Lewis number equal to one, the second term is equal to zero, and Equation 23 simplifies to:

$$
\dot{q}=-\frac{k_{f}}{c_{p f}} \frac{\partial i}{\partial n}
$$

This is the basis for finding that heat transfer analyses based on enthalpies and frozen properties lead for dissociating gases with Lewis numbers not far from unity to simple solutions. For a more detailed discussion, the reader is referred to Lees. 96

\section{B. TEMPERATURE FIELD CREATED BY A STATIONARY HEAT SOURCE}

Equation 17 describes the temperature field in a semi-infinite solid over which a point heat source moves with a constant velocity(v). The corresponding equation describing the temperature field created by a stationary heat source in a semi-infinite solid is:

$$
v=\frac{1}{2 \pi P e_{r}}
$$

The velocity $v$ appears in the parameters on the left and on the righthand side of the equation and cancels out. The equation, however, is written in this form to bring out similarity to Equation 17. It has been mentioned in the text that both equations can also be interpreted as describing the temperature field in the material when a heat flux $\dot{q}$ is distributed over any of the isothermal surfaces. In this way the two equations can be used to obtain an estimate of the reduction in temperature 
increase caused by the movement of the point source by selecting an isotherm in Figure 54, determining an average $\mathrm{Pe}_{r}$ for this isotherm, and calculating the corresponding temperature parameter $v_{s}$ from Equation 26 . The table in the text was calculated in this way. In order to apply this table to the determination of the maximum temperature increase, one may calculate a Peclet number $\mathrm{Pe}_{r}$ with the radius of the arc attachment area at the anode and with the velocity with which the arc moves in downstream direction.

\section{ii. List of Symbols}

\begin{tabular}{|c|c|c|}
\hline Symbol & Unit & Definition \\
\hline $\mathrm{C}$ & -- & constant (depending on flow geometry) \\
\hline$c_{p}$ & $\mathrm{~kJ} / \mathrm{kg}{ }^{\circ} \mathrm{K}$ & specific heat at constant pressure \\
\hline $\mathrm{D}$ & $\mathrm{m}^{2} / \mathrm{sec}$ & diffusion coefficient \\
\hline $\mathrm{D}_{\mathrm{a}}$ & $\mathrm{m}^{2} / \mathrm{sec}$ & ambipolar diffusion coefficient \\
\hline e & $A / \sec$ & electronic charge \\
\hline$h_{i}$ & $\mathrm{~kg} / \mathrm{m}^{2} \mathrm{sec}$ & heat transfer coefficient based on enthalpies \\
\hline I & A & total current \\
\hline$i$ & $\mathrm{~kJ} / \mathrm{kg}$ & total enthalpy \\
\hline j & $A / m^{2}$ & current density \\
\hline k & $\mathrm{kJ} /{ }^{\circ} \mathrm{K}$ & Boltzman constant \\
\hline k & $\mathrm{kW} / \mathrm{m}^{\circ} \mathrm{K}$ & thermal conductivity \\
\hline L & m & characteristic length \\
\hline Le & -- & Lewis number \\
\hline$\dot{\mathrm{m}}_{\mathrm{c}}$ & $\mathrm{kg} / \mathrm{sec}$ & mass flow through constrictor tube \\
\hline $\mathrm{Nu}$ & -- & Nusselt number \\
\hline$n$ & -- & surface normal \\
\hline $\mathrm{Pe}$ & -- & Peclet number \\
\hline $\operatorname{Pr}$ & -- & Prandt7 number \\
\hline$\dot{Q}$ & $\mathrm{~kW}$ & total heat flux per unit time \\
\hline$\dot{q}$ & $\mathrm{kw} / \mathrm{m}^{2}$ & heat flux per unit time and area \\
\hline $\operatorname{Re}$ & -- & Reynolds number \\
\hline$r$ & $\mathrm{~m}$ & distance in polar coordinates \\
\hline$T$ & ${ }^{\circ} \mathrm{K}$ & absolute temperature \\
\hline $\mathrm{T}_{\mathrm{e}}$ & ${ }^{\circ} \mathrm{K}$ & absolute electron temperature \\
\hline
\end{tabular}


List of Symbols (cont'd.)

\begin{tabular}{cc} 
Symbol & Unit \\
\hline$U_{a}$ & $V$ \\
$V$ & $\mathrm{~m} / \mathrm{sec}$ \\
$v$ & $\mathrm{~m} / \mathrm{sec}$ \\
$w$ & -- \\
$x$ & $m$
\end{tabular}

Greek

$\begin{array}{lcl}\alpha & \mathrm{m}^{2} / \mathrm{sec} & \text { thermal diffusivity } \\ \nu & -- & \text { temperature parameter } \\ v_{\mathrm{S}} & -- & \text { temperature parameter (stationary) } \\ \mu & \mathrm{kg} / \mathrm{m} \mathrm{sec} & \text { dynamic viscosity } \\ \rho & \mathrm{kg} / \mathrm{m}^{3} & \text { density } \\ \Phi & v & \text { work function }\end{array}$

Subscripts

A

a

$a b 1$

conv

e

$\mathrm{f}$

I

j

$r$

ra

$r a+c$

re

$s$

w

$\mathrm{x}$

$\infty$

$\Phi_{\mathrm{a}}$
Definition

anode fall potential

gas velocity outside the boundary layer

velocity of heat source

mass fraction of one component

distance in Cartesian coordinates

going to the anode

property of the anode (except in $D_{a}$ )

ablative

convective

at the outer edge (except in $\mathrm{Te}$ )

"frozen"

related to the total current

related to current density

based on a length in polar coordinates

radiative (absorbed)

radiative and convective

radiative (emitted)

going to a segment (except in $v_{S}$ )

at the wall or surface

based on a length in Cartesian coordinates

at $r=\infty$

related to anode work function 


\section{PLASMA ENERGY TRANSFER TO A MOLTEN SURFACE ${ }^{122^{*}}$ \\ 1. Plasma Heat Transfer With Current Flow}

The interactions of the various arc processes going on with a molten anode spot make a very difficult job of developing an equation to describe plasma heat transfer into the anode. Tests were used to determine the value of the heat input to the workpiece and its dependence upon both the arc current and arc length. In both cases an approximately linear relationship was found; the curves for copper are shown in Figure $61 \mathrm{~A}$ and $\mathrm{B}$, and the values of the constants for the equation representing the results obtained

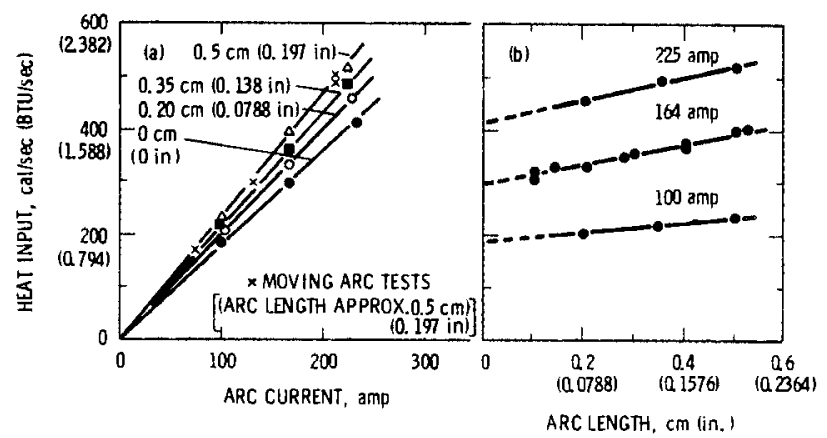

FIGURE 61A \& B. Copper: Relationship Between Heat Input (a) Arc Current for Different Arc Lengths

(b) Arc Length for Different Arc Currents 122

*This section reproduces approximately one page of material from an article by R. L. Apps and D. R. Milner - "Heat Flow in Argon-Arc Welding," British Welding Journal, Vol. 2, No. 10, 108, pp. 477-478, 1955. This article is a report of the authors' work at Birmingham University, sponsored by the U. K. Atomic Energy Authority, Harwell Research Establishment. 
with other metals are given in Table 4.122 The heat-input/arc-length curves have been extrapolated to zero arc length enabling a relationship to be derived for the heat input in terms of current and arc length, since preliminary work has suggested that the heat input at zero arc length would be an important factor.

Calorimetric measurements of the heat input obtained in actual weld runs were made. Results showed good agreement with the values obtained with a stationary arc although experimental accuracy was lower because of the difficulty in controlling the arc length during a welding run (Figure 61A). 122

The extrapolation of the heat-input/arc-length curves to zero arc length allows the heat input to be expressed as the sum of two terms, one depending solely on arc current and the second being dependent on both arc current and arc length:

$$
q=a I+b L I
$$

TABLE 4. Values of Constants in the Heat-Input Equation 122

\begin{tabular}{|c|c|c|c|c|}
\hline METAL & $A, V$ & RANGE OF $b, V$ & MEAN $b, V$ & $\mathrm{p}$ \\
\hline Aluminum & $7 \cdot 1$ & $1 \cdot 8-2 \cdot 5$ & $2 \cdot 2$ & $0.31-0.44$ \\
\hline Armco Iron & $7 \cdot 3$ & $4 \cdot 2-4 \cdot 9$ & $4 \cdot 6$ & $0.60-0.78$ \\
\hline Nickel & $6 \cdot 6$ & $3 \cdot 4-4 \cdot 2$ & $3 \cdot 7$ & $0.44-0.56$ \\
\hline Copper & $7 \cdot 6$ & $4 \cdot 2-5 \cdot 2$ & $4 \cdot 5$ & $0.43-0.67$ \\
\hline Lead & $6 \cdot 7$ & $2 \cdot 3-2 \cdot 9$ & $2 \cdot 7$ & $0.46-0.56$ \\
\hline Copper & $8 \cdot 0$ & $4 \cdot 4-4 \cdot 8$ & - & $0.50-0.75$ \\
\hline
\end{tabular}


where $q=$ heat input to the workpiece of any length $L$,

$I=\operatorname{arc}$ current

$a=$ constant depending on metal comprising work piece, and

$b=$ constant, possibly variable with current

There is some indication that $b$ varies with current, particularly in the low current range. Between 40 and $200 \mathrm{~A}$ this variation is so small as to be effectively constant. The mean value was sufficiently accurate for use in heat flow experiments. Values of $a$ and $b$ for five metals are summarized in Table 4. Equation 27 permits an interpretation of the experimental results in terms of arc mechanism. The large term, aI, which gives the current at zero arc length can be interpreted as the heat input due to electron current, which enters the anode through the very small area of the anode spot. The second term, bLI, may be attributed to the heat received from the positive column by either radiation or gaseous conduction.

The experimental error is only a small fraction of the total heat input but is a significantly larger percentage of the positive column heat input alone, particularly since the latter is obtained by the subtraction of the zero arc length value which is itself obtained by extrapolation. However, the error in the value of the heat input at zero arc length should be small, and this is the quantity which is important in the present work.

Bearing in mind this possible error, an estimate was made of the proportion of the total energy expended in the positive column which is being absorbed as heat by the anode. The total heat generated in the positive column:

$$
H_{T}=E L I
$$

where $E$ is the potential gradient of the positive column, Then from Equation 27, the proportion $p$ of this heat that is being absorbed by the anode is:

$$
p=\frac{b}{E}
$$


However, in some cases the potential gradient varies with the current and this may in fact account for the variation of $b$ with current. Curves relating $E$ and $I$ are given in Figure $62^{122}$ and these have been used to derive the values of $p$ that are included in Table 4.122

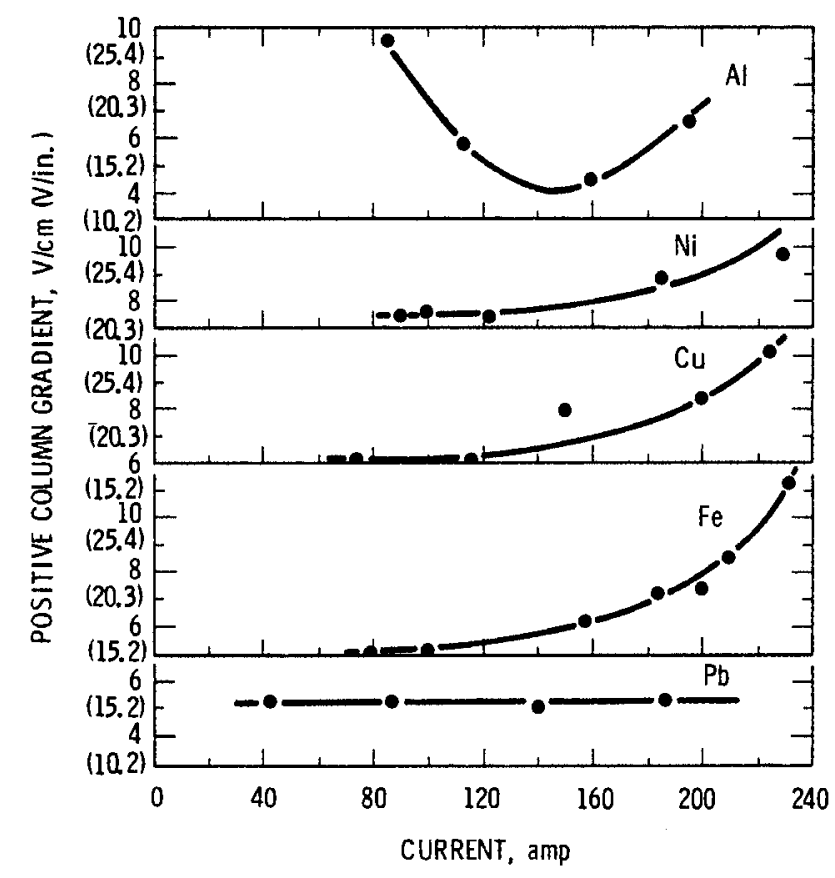

FIGURE 62. Variation of Positive Column Potential Gradient With Arc Current 122 


\section{WELDING POWER SUPPLIES}

\section{A. WELDING POWER SUPPLY CHARACTERISTICS}

The work performed in determining the suitability of welding power supplies for precision welding, both direct- and alternating-current, was directed toward the improvement of end closure welding for nuclear fuel elements and fabrication of ordinance components. $123,124,125$ The lengths of the welds for nuclear fuel (all of those involved were circular) vary from about one inch to about eight inches. Arc starting required that minimal tungsten was blown down into the welds. In addition, the current had to be decayed at the closure point until it would not leave a crater when the arc was extinguished. The weld surface had to be very smooth and inspectable for minute defects.

All of the work with direct- and alternating-current was performed with the gas-tungsten-arc with the direct-current used on aluminum, copper, and Zircaloy-2. Alternating-current was used exclusively on aluminum.

Requirements of the welding process were:

- Good arc starting

- Smooth readily inspectable weld surfaces

- Good alloying of the braze metal and aluminum cladding into a weld

- A crater fill that was free of craters and cracks

The ordinance components were of a single geometry of many designs.

\section{Direct-Current Welding Power Supplies}

Single-range direct-current welding power supplies available on the market today, as far as is known, are of the six pulse type. ${ }^{126}$

For one complete phase rotation (from the time a phase starts to conduct in a positive direction until that phase starts to conduct again in the positive direction), six half cycles or pulses of current of the same polarity have been delivered to the welding circuit.

Note: Whenever "cycles" is used by itself, it refers to 60 cycles per second. *For recent developments, see Addendum. 
There are four types of rectifier circuits currently employed:

Magnetic amplifier, three-phase, full-wave bridge

Thyristor, three-phase, full-wave bridge

Thyristor, six-phase star, half-wave

Transistor, three-phase, full-wave bridge

A six-phase full-wave bridge is available on special order. In Figure 63 are shown for comparison the unfiltered full-wave currents of two-, three-, six-, and twelve-pulse rectifier systems. However, the ripple may be increased by the type of current control system used with the rectifier, i.e., saturable reactor, magnetic amplifier, thyristor, or transistor controlled. The transistor has the capability of valving or throttling the output current of the rectifier without affecting the amount of ripple or open-circuit voltage. However, with the judicious inclusion of inductance in the current carrying circuit, the output current ripple over the full welding current range can be set at any desired pattern

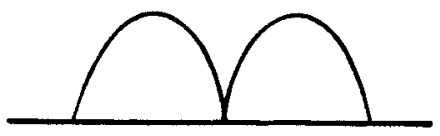

2 Pulse System

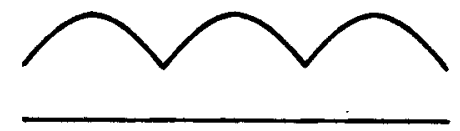

3 Pulse System

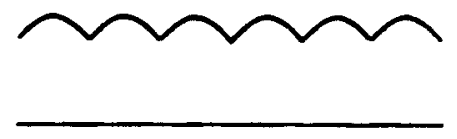

6 Pulse System

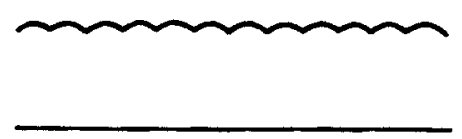

12 Pulse System

FIGURE 63. Ripple in the Full-Wave Current

of 2-, 3-, 6-, and 12-Pulse Rectifiers 
between the unfiltered, throttled current to the equivalency of the output current of a motor generator set. The inductance is designed by the manufacturer to produce optimum current rise and decay times required for optimum arc starting, current programming, and crater filling for the rectification circuit employed. Additional inductance to reduce current ripple beyond that supplied by the manufacturer, of either the saturating or non-saturating types of inductance, to the welding current circuit does nothing to improve and may somewhat inhibit arc starting. It may also decrease the time rate of rise of the ignition current as there must be current flowing through the inductance before there is any reduction in the peak to valleys of the current and thus of the voltage. (Pulsing current is treated in a following section.) The point of maximum current ripple on the current range of all commercially available welding power supplies may vary from the minimum current to three-quarters of the maximum current. Its exact ultimate of maximum or minimum current ripple, to some extent, is determined by the arc voltage and the electrode size-current relationship. The excess thermal electron emission produced by thermal lag in the tungsten electrode, as the current decays rapidly, permits the maintenance of the arc with a lower instantaneous open-circuit voltage than when the electrode temperature is lower at the time current decay starts.

In order to obtain reproducible results that may be compared with various direct-current power supplies studied, all of the oscillograms were taken with a resistance load in place of an arc. For each power supply, the same value of current per step was used with a load potential of 30 volts as read with a D'Arsonval type voltmeter for all steps and power supplies.

In order to have a definition of current ripple which may be measured, is meaningful for a welding arc, and readily understood, the following definition has been devised: The ripple in the welding current is the ratio of the difference between the maximum peak and the minimum value of the current during $1 / 60 \mathrm{sec}$ to the value as read with a D'Arsonval type ammeter (Figure 64). The peak to valley difference can be determined by either an oscilloscope or a light beam oscillograph. The response time of a direct writing oscillograph is too slow to obtain a meaningful value. 


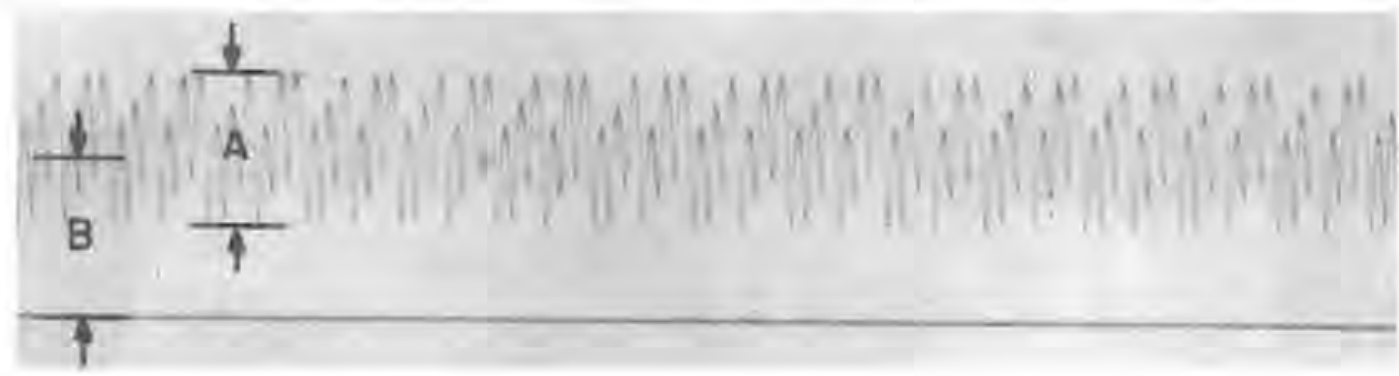

Percent Ripple $=\frac{A-\text { As Read with Oscillograph }}{B-\text { As Read with D' Arsonval Ammeter }} \times 100$

\section{FIGURE 64. Definition of Current Ripple}

The open-circuit voltage required to produce a stable arc is determined by the ratio of the depth of the current valley to the residual current and the time-width of the valley. This applies to both direct-and alternatingcurrents.

The magnetization of the inductive circuit during current rise is driven by the welding current while during current decay the magnetization coasts down. Thus, the time for free current rise will be less than the time for free current decay. The time ratio of decay to rise may be as great as two to one.

\section{a. Power Supplies Studied}

i. Magnetic Amplifier. Oscillograms of the welding current of an early model three-phase, half-wave, magnetic amplifier power supply with heavy inductance which delivers three current pulses per cycle are shown in Figure 65. When welding aluminum at 100 amperes with helium as a shielding gas, the arc becomes a series of discharges, making a high pitched squealing noise when the current is decayed to 10 amperes and below. The welding current is a series of discharges producing crescent shaped pulse patterns on the weld surface as they overlap. It is obvious that the unit has 


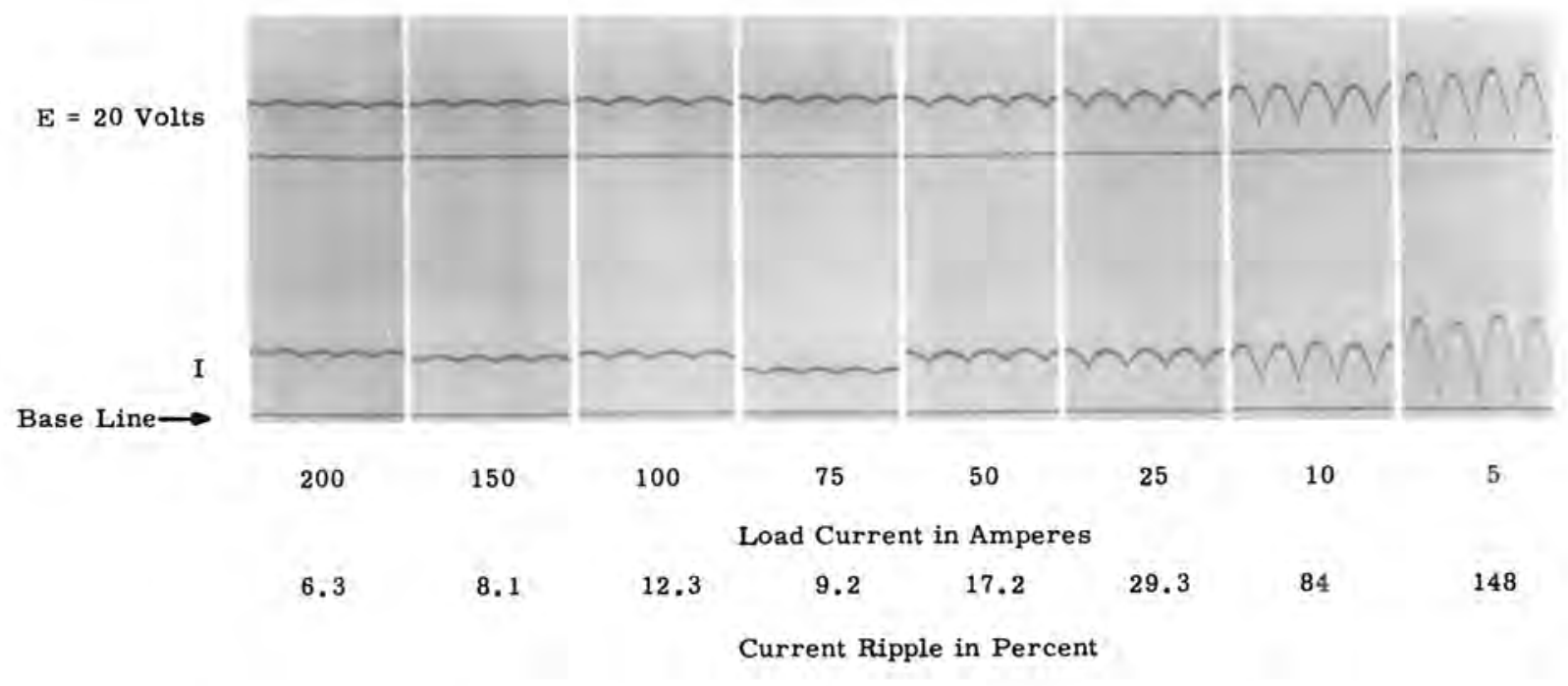

FIGURE 65. Oscillograms of Three-Phase Half-Wave Magnetic Amplifier Rectifier Showing Amount of Ripple

adequate open-circuit voltage as the arc does not go out. To provide fast positive arc starting it has a hot start circuit. By reducing the inductance the ripple was increased to 86 percent at 150 amperes for studies of the effect of current ripple on welding aluminum clad nuclear fuel elements.

In Figure 66 are shown the oscillograms of a three-phase, ful1-wave, magnetic amplifier type power supply which delivers six current pulses per cycle. The magnitude of the current ripple is so great that it was not possible to weld aluminum using the gas-tungsten-arc process with helium as a shielding gas. Over the current range from 250 to 150 amperes the minimum value of voltage available to keep the arc burning is approximately six volts which is less than the burning voltage of an inert gas tungsten welding arc. Arc hysteresis plus the open-circuit voltage keeps the arc burning. The addition of inductance and balancing of the magnetic amplifiers would reduce the ripple, improve the quality of the welds and arc performance, and reduce the quantity of shielding gas required. The time rate of 


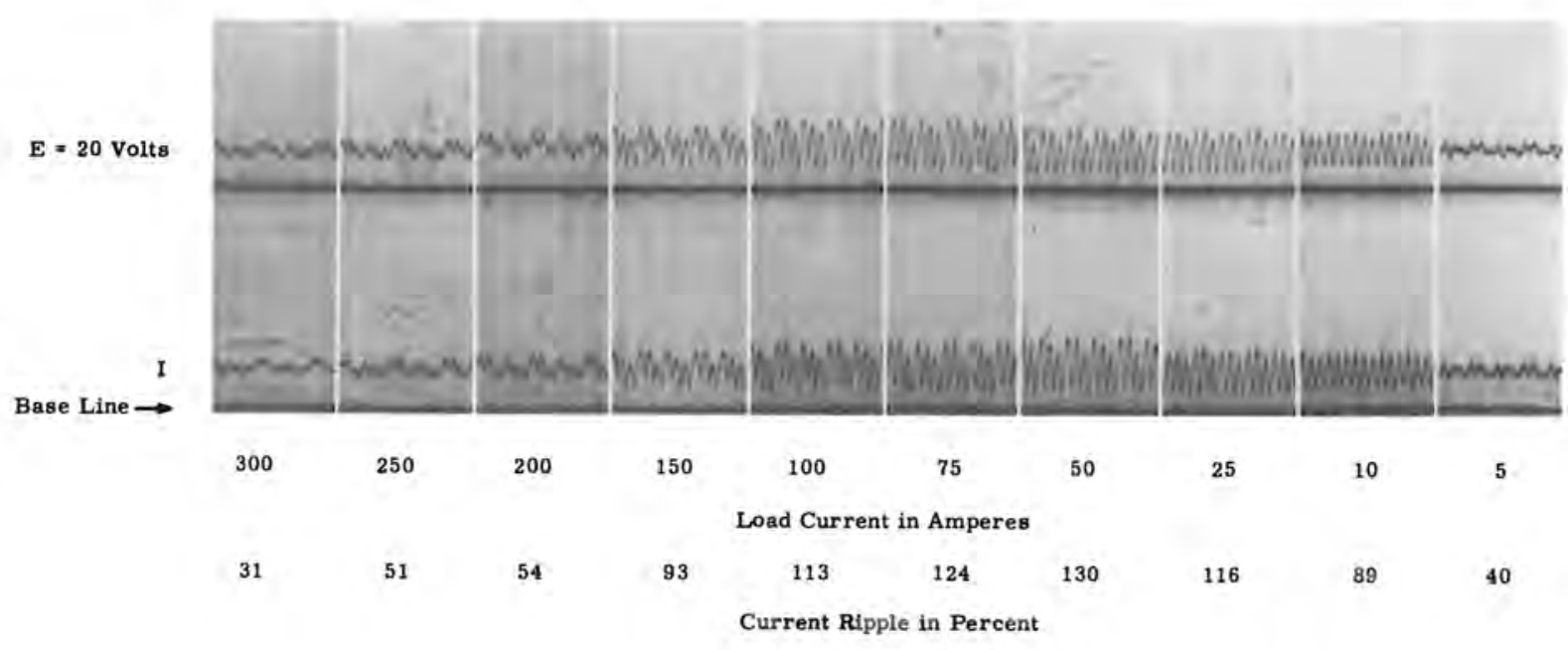

FIGURE 66. Oscillograms of Three-Phase Fu11-Wave Magnetic Amplifier Rectifier Showing Amount of Ripple

rise and decay of current in magnetic amplifiers is usually less than ten cycles so that the addition of a small amount of inductance to reduce the ripple would not affect the performance an appreciable amount.

The characteristics of a three-phase, full-irave bridge magnetic amplifier rectifier delivering six current. pulses per cycle are shown in the oscillograms of Figure 67 . Though its current ripple is exceptionally high, its 150 volts open-circuit gives it good arc starting with helium, using an impulse arc starter, and good arc stability. Its welding speed and weld surface finish are good. There is a lack of balance between the magnetic amplifiers as shown by the variation of the heights of current peaks in series of threes. Balancing of the magnetic amplifiers and the addition of some inductance will greatly improve the overall performance.

In Figure 68 are shown the oscillograms of a commercially available power supply using a three-phase, full wave, maqnetic amplifier rectifier delivering six pulses per cycle. When welding aluminum with the inert 


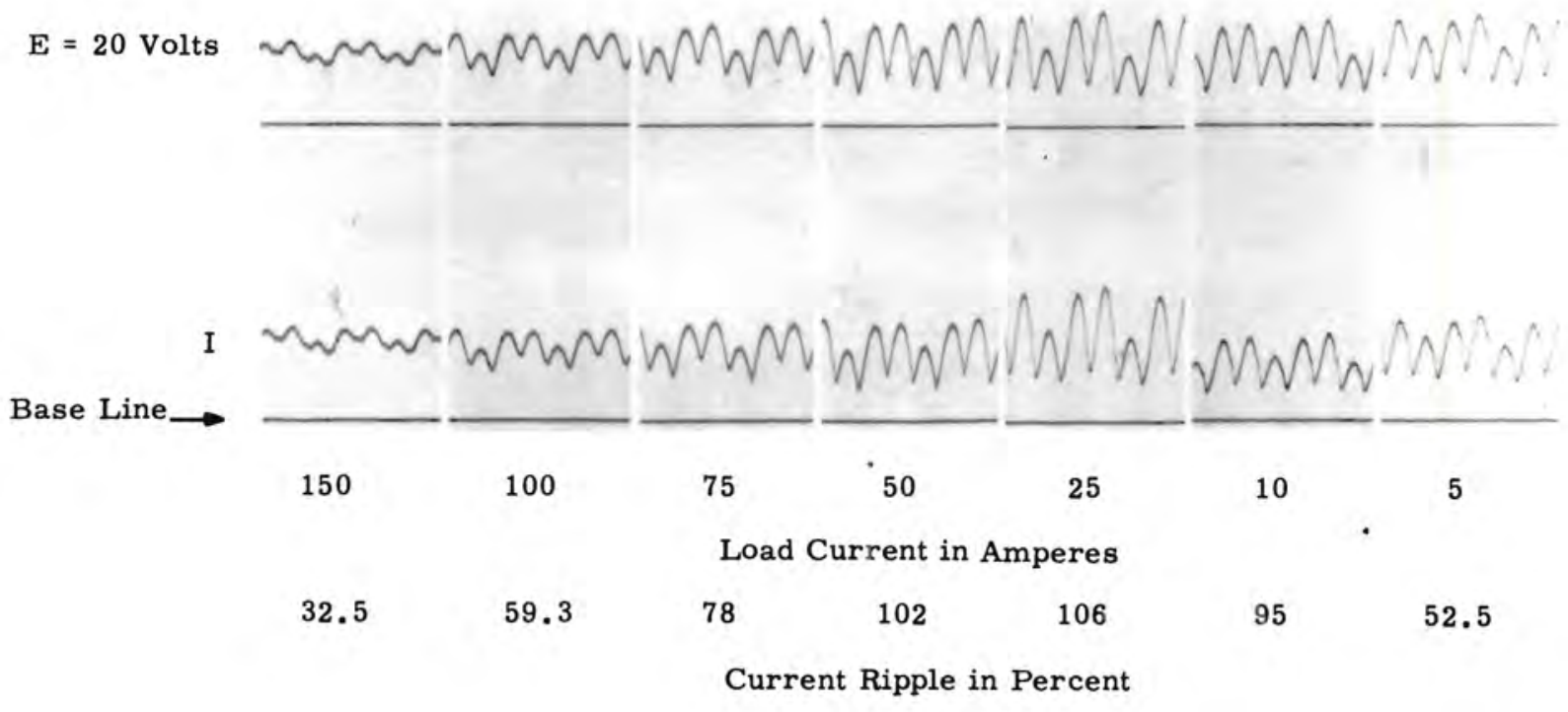

FIGURE 67. Oscillograms of Three-Phase Fu11-Wave Magnetic Amplifier Rectifier Showing Amount of Ripple

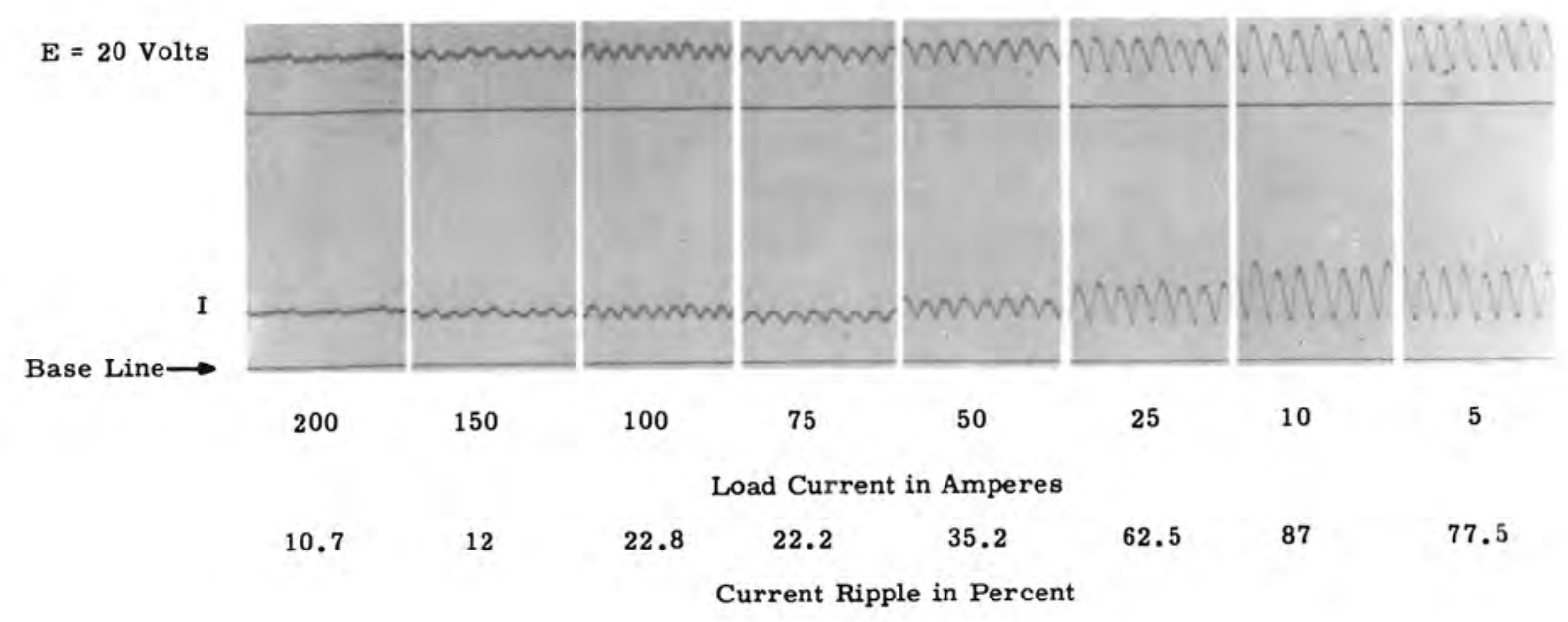

FIGURE 68. Oscillograms of Three-Phase Fu11-Wave Magnetic Amplifier Rectifier Showing Amount of Ripple 
gas-tungsten-arc process at 100 amperes and helium shielding gas, the arc will go out if decayed to currents below 10 amperes. In addition to this, the arc is hard to start. By connecting two of these units in series the arc starts well and will not go out at minimum current. But the arc will squeal, becoming a series of discharges. Experience with these units has shown that their performance can be improved considerably by the addition of inductance, but the arc will go out at low values of current. The unit has a hot start circuit.

This unit was used for the studies of the effect of current ripple on welding aluminum clad fuel elements at current ripples of 2, 20, 26, and 40 percent by changing the amount of inductance in the welding current circuit. The two lower values of current ripple produced excellent welds.

The unit has also been used for the studies of welding copper and Zircaloy-2 using argon as the shielding gas with good results.

Theoretically, the twelve pulse per cycle system is most advantageous for welding as the natural ripple of the system approaches that of a motor generator set. Therefore, it does not require additional inductance to commutate over the valleys in the current ripple.

A twelve-pulse system that was studied used parallel three-phase, fullwave bridge magnetic amplifiers. The insulating transformer of one rectifier was connected wye-delta and the other delta-delta to produce the 30 degree voltage phase shift between the two units required to produce twelve pulses of current for a complete phase rotation in one cycle. Secondaries of the rectifiers were connected in parallel. No additional inductance was used in the welding current circuits. Series operation does not produce the equivalent of twelve pulses as four of the magnetic amplifiers are in series during each conduction period. The unit had an open-circuit potential of 80 volts. Arc starting tests at 5 amperes with helium shielding, a $1 / 16$-inch $(1.58 \mathrm{~mm})$ two percent thoriated tungsten electrode, aluminum workpiece, and high-frequency starter produce positive repetitive arc starting with a cold electrode without cleaning the electrode or aluminum between arc starts. It appears that the low value of inductance 
and the short time between current pulses are responsible for the improved arc starting. These units are available only on special order. An interphase transformer is required for parallel operation of two power supplies for twelve pulse operation.

ii. Thyristor Controlled Rectifier. The optimum of what can be done with a rectifier type power supply is shown in the oscillograms of Figure 69 . In this case the circuit was a six-phase, half-wave thyristor controlled rectifier with judiciously designed saturating inductance which delivers six pulses of current per cycle. It is available in open-circuit voltages from 80 to 150 volts and, if desired, with a tap switch to select a particular open-circuit voltage. Other voltages may be obtained on special order. A combination of selectable open-circuit voltages for arc starting and welding may also be obtained. There is a hot start device. Its arc starting and stability characteristics are exceptionally good. In addition, the times for the current to rise from minimum to maximum and to decay from

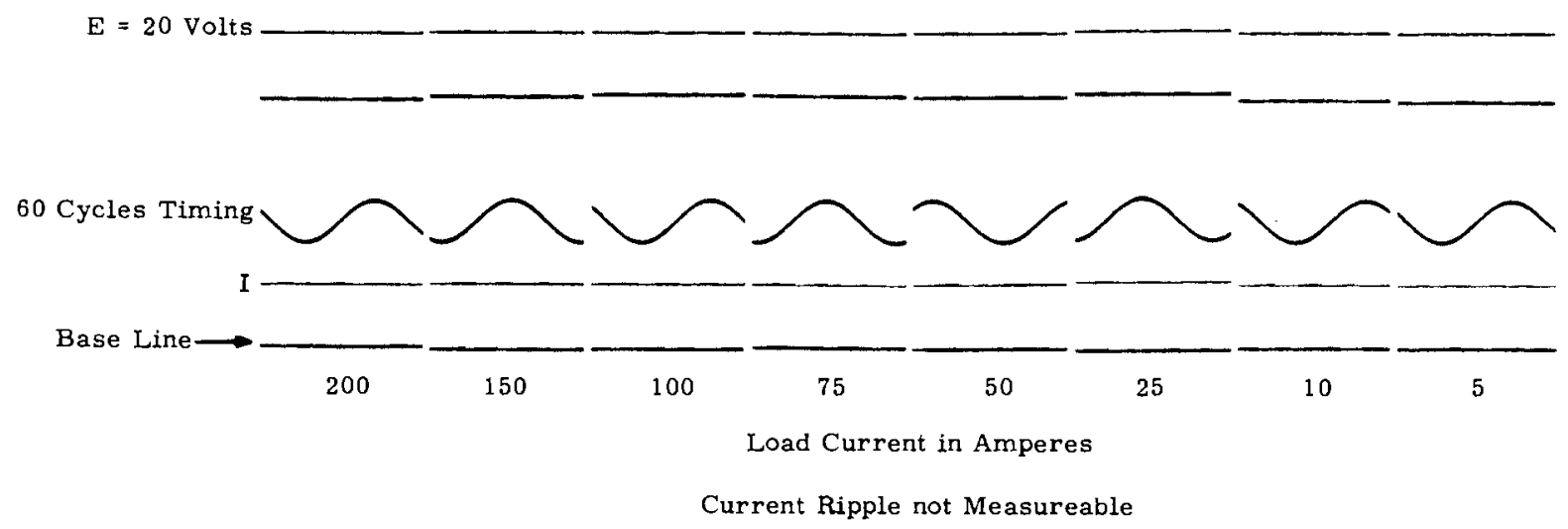

FIGURE 69. Oscillograms of Six-Phase Half-Wave Silicon Controlled Rectifier Showing Amount of Ripple 
maximum to minimum are five cycles which exceed the requirements for excellent current programming. The surface finish of the welds is equal to that of a motor generator. Also the arc will remain stable when decayed from maximum to minimum current when welding aluminum with helium using the gas-tungsten-arc process. It does very little to improve arc starting using a high frequency starter when helium is used as the shielding gas.

This unit has been used extensively for argon shielded gas tungsten arc welding development studies of Zircaloy-2 with excellent results.

\section{b. Basic Findings}

- Arc starting with argon is improved by reducing the ripple in the open circuit voltage.

- Arc stability over the full current range is provided by some minimal value of ripple in the current in relation with the open circuit voltage, the inductance, and the number of pulses of current per cycle.

- Optimum current programming is provided by current rise times from minimum to maximum and maximum to minimum in the order of five to thirty cycles depending on the ripple requirements.

- Welding speed is increased and surface finish is improved by decreasing the amount of ripple in the current.

- The maximum amount of ripple at any point over the full current range for maximum welding performance appears to be in the order of 10 to 20 percent.

- Within the desirable limits of current ripple, 10 to 20 percent, it has essentially no affect on alloying $x 8001$ aluminum brazed with $A-13$ alloy and welded. Between 86 and 2 percent ripple there is a 19 percent improvement in alloying. 123

- There was a 70 percent increase in cracking of $x 8001$ aluminum-A-13 aluminum brazed joints welded with helium shielding gas as current ripples increased from 2 to 86 percent. 123

- Helium shielding gas requirements for welding $x 8001$ aluminum-A-13 alloy aluminum brazed joints were increased 114 percent at 150 amperes when going from 2 to 86 percent current ripple. ${ }^{123}$ 
- High quality bead on plate welds can be made with direct-current electrode positive and argon shielding gas with a current ripple of one percent. Increasing the ripple degrades the weld quality. ${ }^{123}$

- Zircaloy-2 when welded with argon and a 15 percent current ripple produces very high quality welds.

- Direct-current welding power supplies are available with open-circuit voltages, current ripple, current rise and decay times, logic controls, numerical tape, ${ }^{127}$ and computer programming ${ }^{128}$ to fit the most demanding requirements.

\section{Pulsed Current Direct-Current Welding Power Supplies*}

\section{a. Pulsed Direct-Current Welding Characteristics}

Pulsing of welding current from a high value to a low value was developed to produce precision and high quality welds between two thin pieces of material, a thin and a thick piece of material, or non-uniform material thickness along a joint. An example is the root pass in pipes or plate joints to be welded.

There are three types of arc characteristics used in pulsed current gas tungsten arc welding: ${ }^{129}$

- Soft arc produced with low values of current for the tungsten size used.

- Hard arc produced with high values of current for the tungsten electrode size used.

- Overshot arc has a spike of current continuing as the peak current reaches its value that may be equal to or greater than the peak current.

Figure 70 is a schematic representation of an ideal pulsed current rectangular waveform showing the associated pulse parameters. For this waveform, the average current $I_{a v}$ is equal to:

$$
I_{a v}=\frac{I_{B} t_{B}+I_{p} t_{p}}{t_{B}+t_{p}}
$$

*For recent developments, see Addenda I and II. 


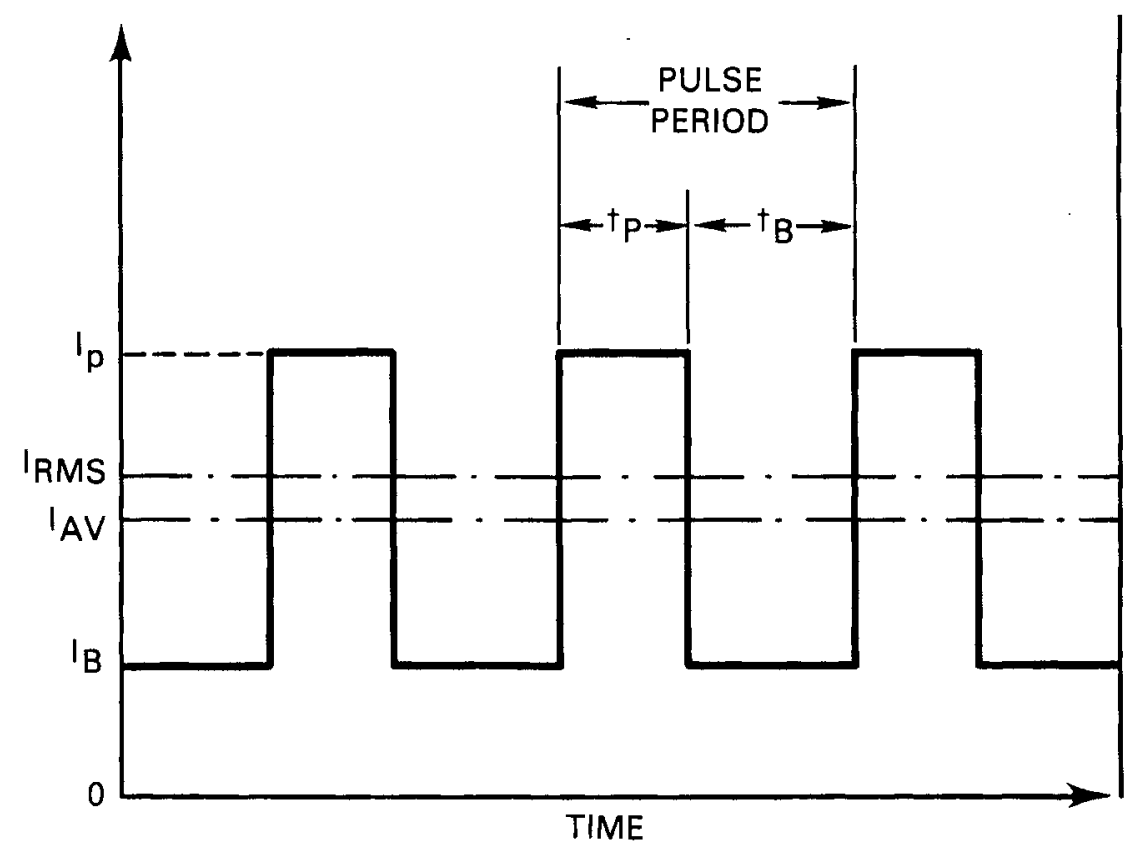

Figure 70. Schematic of an Ideal Pulsed Current Square Wave Form and Associated Pulse Parameters 129

where $I_{B}$ and $I_{p}$ are the background and peak currents and $t_{B}$ and $t_{p}$ are their respective durations. The effective current $I_{\text {eff }}$ or the root-meansquare $I_{\text {RMS }}$ is:

$$
\begin{aligned}
& I_{\text {eff }}=I_{R M S}=\left(\frac{I}{t} \int_{0}^{t} i^{2} d t\right)^{1 / 2} \\
& I_{\text {eff }}=I_{\text {RMS }}=\left(\frac{I^{2} t+I^{2} t}{t}\right)^{1 / 2}
\end{aligned}
$$

where $t$ is the time of the pulse cycle and is equal to:

$$
t=t_{B}+t_{p}
$$


The pulse frequency $f$ is defined as: ${ }^{127}$

$$
f=\frac{1}{t}=\frac{1}{t_{B}+t_{p}}
$$

In addition to the above pulse parameters, the varying pulse time ratio is $t_{p} / t_{b} \cdot 127$

In Figure 71 is a representative of a general purpose pulsed current rectangular wave form with its three phase $60 \mathrm{~Hz}$ ripple showing the additional pulse parameters. 130 The average current, $I_{a v}$, equation for the background and peak currents are the same as for the ideal rectangular wave form with the addition of the current rise, $I_{R}$, and the current decay, $I_{D}$, so the equation for the average current becomes:

$$
I_{a v}=\frac{I_{B} t_{B} \div I_{p} t_{p}+I_{R} t_{R}+I_{D} t_{D}}{t_{B}+t_{P}+t_{R}+t_{D}}
$$

However, since there is a ripple in all of these currents, it becomes a complex problem to calculate them directly. The base and peak currents can be measured directly with a D'Arsonval (regular) direct-current ammeter. The rise and decay currents can be calculated from an oscillogram of these currents by averaging the current ordinates over each time period.

The effective current equation becomes:

$$
\begin{aligned}
& I_{e f f}=I_{R M S}=\left(\frac{I}{t} \int_{0}^{t} i^{2} d t\right)^{T / 2} \\
& =\left[\frac{T}{t} \int_{0}^{t}\left(I_{B}^{2} t_{B}+I^{2} t_{P}+I_{R}^{2} t_{R}+I_{D}^{2} t d\right) d t\right]^{1 / 2}
\end{aligned}
$$




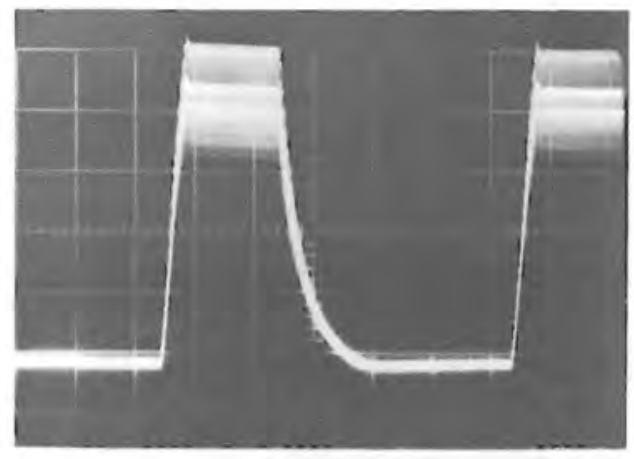

300 AMPS MAX

30 AMPS MIN

0.5 SECIDIV

60 AMPS/DIV

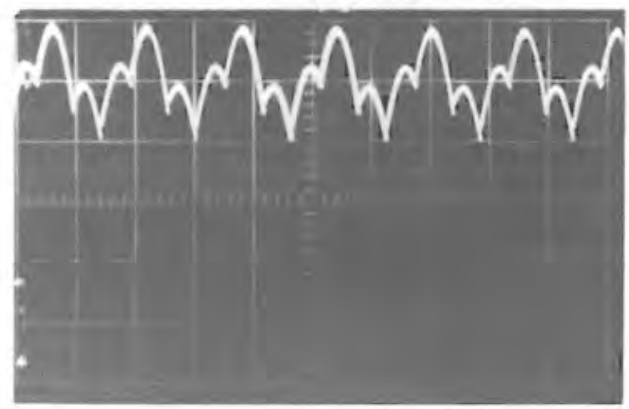

3 PHASE RIPPLE

300 AMPS

5.0 MILLISECONDSIDIV

60 AMPS/DIV

FIGURE 71. A Representative General Purpose Pulsed Current Square Wave Form and Its Three Phase $60 \mathrm{~Hz}$ Ripple ${ }^{130}$ 
The terms for the effective currents cannot be integrated until the equations for the instantaneous values of each current are known. The easiest way to calculate the RMS value of these currents is to calculate them from a light beam or cathode vary oscillogram by taking the square root of the sum of the squares of the current ordinates over the time period of each current section. Effective values of current for the base and peak currents can be measured with an iron vane or dynamometer type ammeter.

In Figure $72^{129}$ is shown a hybrid pulse of current with its narrow high peaked spike starting to form as the peak current reaches its value, continuing to rise to its peak value, and then decaying to the value of the peak current. ${ }^{129}$

By programming the current control of the welding power supply, with numerical or computer programming, it is possible to provide a "hybrid" current program. This is done by taking the characteristics of soft arc,

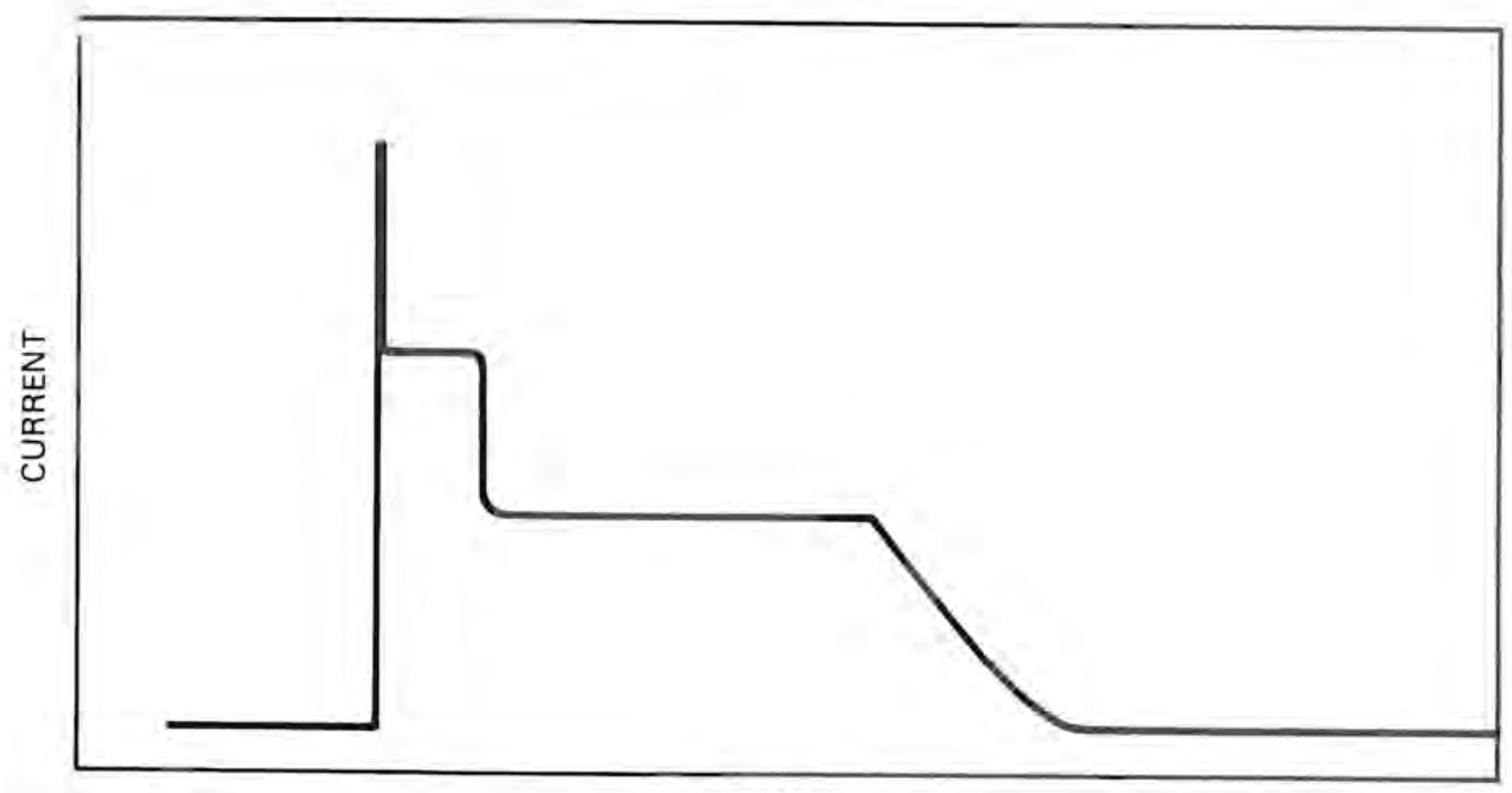

TIME

FIGURE 72. Hybrid current Pu1se $\mathrm{s}^{129}$ 
hard arc, and the overshot arc and programming them to occur at the proper point along the weld joint to take advantage of their superior characteristics to produce a better quality weld.

Though a number of investigators have explored the area of pulse shapes in relation to the quality of the weld produced, there has been no general agreement on the selection of pulse shapes. It is beginning to be felt that for precision reproducible welding that the essentially straight sided current pulses are required. 131

There are two basic types of pulses termed "hard" and "soft" and each has its area of application in precision welding. Figure 73 shows the relationship between pulse current and duration for a given penetration requirement, and the current is identical for both plain and overshot current leading edges. The curves in Figure 73 apply equally well when using either plain or overshot leading edges on initiating the peak current

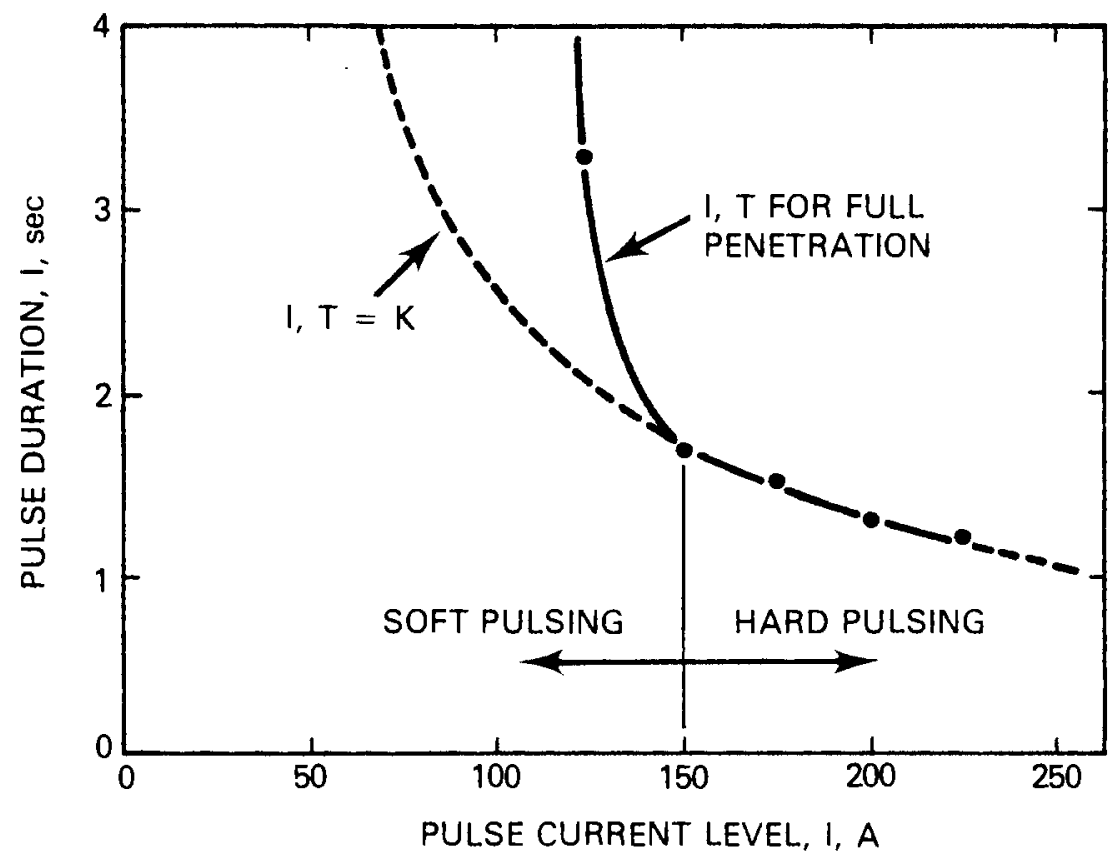

FIGURE 73. Relationship Between Pulse Current Amplitude and Duration For Ful1 Penetration on $3.5 \mathrm{~mm}$ (0.14 in.) Stainless Steel. Square Wave Pulses, Bickground Current $=15 \mathrm{~A}$, Background Duration $3 \mathrm{Sec} .137$ 
pulse. The curve in Figure $73^{131}$ can be considered to have two parts, steeply drooping and shallow drooping. The steeply drooping or low current end of the curve is where the rate of advance of the fusion boundary through the work is slow in comparison with the thermal constant of the material (reciprocal of thermal diffusivity), i.e., this is a "hot soak" situation which is somewhat contrary in principle to the pulsed gas tungsten arc process. The remaining part of the curve, or high current end, is where the rate of advance of the fusion boundary is relatively fast. This part of the curve approximates very closely to a curve of the product of current and time being constant implying that the heat input to the work is also constant. 131

The low current end is referred to as soft pulsing and the high current as hard pulsing. At the moment, the dividing line between hard and soft pulsing has to be determined empirically for each class of application and material. 131

Soft pulses have their area of application when the depth of penetration is greater than about $4 \mathrm{~mm}$ ( $0.16 \mathrm{in}$.$) and where the surface oxides on the$ plate have some peculiar, if yet unclassified, factors. Generally, a pulse height is chosen which gives a duration (the two are interrelated, Figure 73) which is long enough to negate the effects of the variable stepping phenomena as the molten pool grows, but short enough to minimize the heat input. 131

In all other cases, hard pulsing of current is used since these give the lowest total heat input. There appears to be no preferred level of hard pulsing from a heat input viewpoint; however, Figure 74 shows that the top width of the bead can be minimized by keeping to the lower current end of the hard pulsing range.

The finite pulsing program must be determined empirically for each class of application and material. For precision and difficult weldments with variable sections and thickness, a current rise time of one percent of the peak pulse duration is considered adequate. In addition to this, it has been found that if the initial current rises above the steady state value of the current pulse, a profound effect on tolerance in terms of 


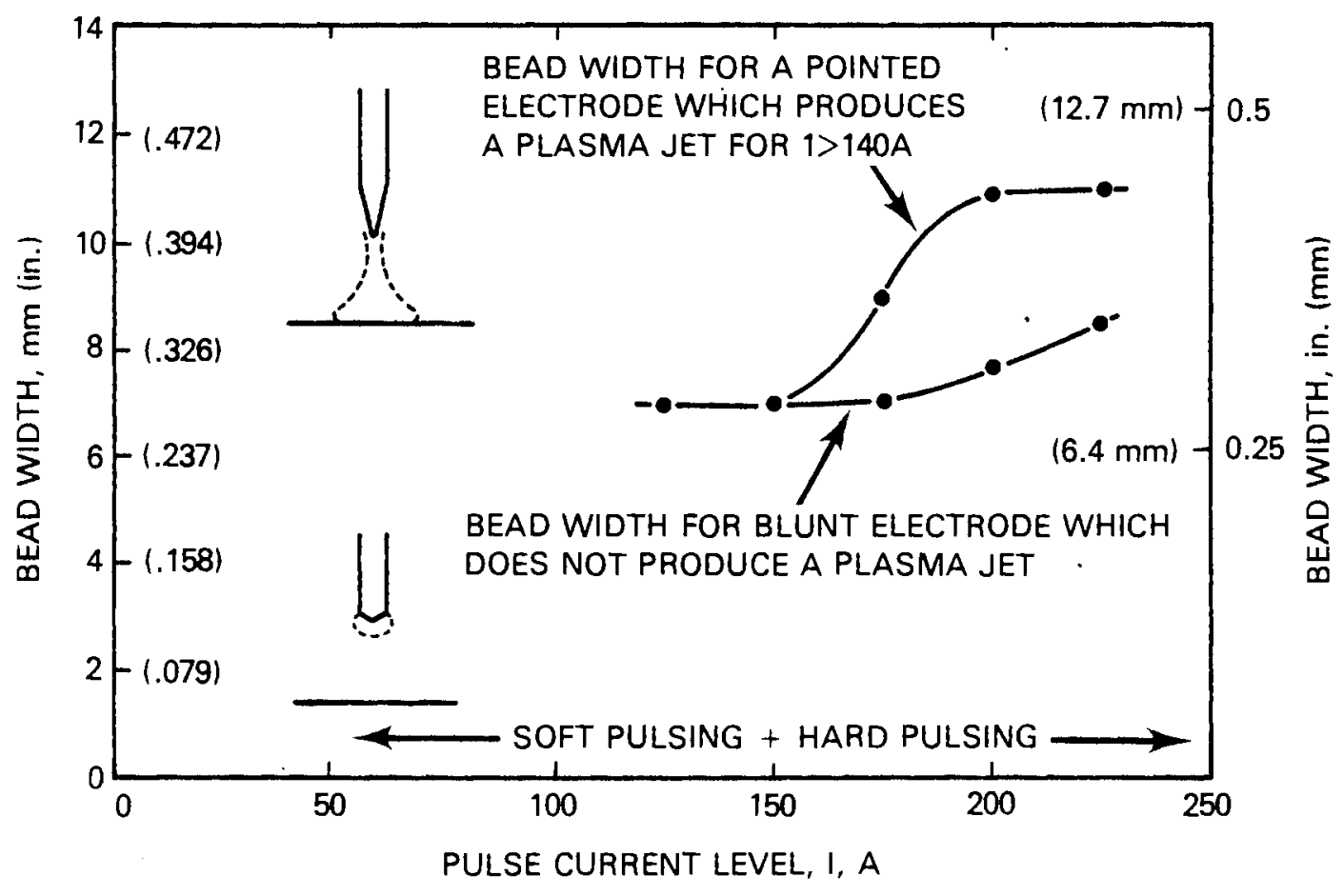

FIGURE 74. Top Width of Weld Beads for Full Penetration On $3.5 \mathrm{~mm}(0.14 \mathrm{in.})$ Stainless Stee 131

penetration are produced. For example, the difference between "justpenetrated" and "fully penetrated" in terms of pulse duration can be minimized by using initial currents which overshoot the normal pulse amplitude by, say $100 \%$. The duration of the overshoot should be in the order of a few milliseconds. 131

b. Power Supplies Studied

There are four different types of gas-tungsten-arc welding power supplies used for pulsed-current welding:

i. Saturable reactor controlled

ii. Magnet amplifier

iii. Silicon controlled rectifier

iv. Transistor controlled rectifier 
i. Saturable Reactor. The saturable reactor produces a variable pulse rate with the current pulse rise and decay slopes being exponential and fixed by the physical characteristics of the current carrying circuit. The current rise time is of much shorter duration than the decay time. In this type of welding power supply, by judiciously selecting the high and low values of current and pulse lengths, a square pulse of current can be effectively produced. This limits the maximum pulse width and amplitude differences for a square current pulse without seriously degrading the pulse geometry. They are generally used where relatively low pulsing rates are adequate.

ii. and $i i i$. Magnetic Amplifier and Silicon Controlled Rectifiers. The second and third types are essentially the same as the first type but have a higher pulsing rate and higher peak pulse to base pulse current ratios, as the current rise and decay times are much shorter.

iv. Transistor Controlled Rectifier. The transistor controlled pulse rate rectifier produces virtually a square wave current pulse. The current pulse remains virtually square regardless of the pulse width or height and the peak and base values of current. Rates of rise and decay of pulse current in the order of $10^{6} \mathrm{~A} / \mathrm{sec}$ are readily maintained with a response time of $10^{7} \mathrm{~A} / \mathrm{sec} .{ }^{132}$ This rate will be reduced if the thermionic emission of the tungsten cathode during current rise cannot follow the emission demand of the current (see arc hysteresis). From minimum to $1 \mathrm{kHz}$ pulsing rates at maximum current and 80 percent current pulse height, the square pulse configuration may be maintained at $10^{6} \mathrm{~A} / \mathrm{sec}$. From $1 \mathrm{kHz}$ to $10 \mathrm{kHz}, 99$ percent of the pulse height may be obtained in less than $50 \mathrm{\mu} / \mathrm{sec} .^{132}$ (These are accomplished by setting the peak values of current and shunting through a resistor with transistors, a portion of the peak pulse current to reduce the peak to this base pulse value.) Between 1 and $10 \mathrm{kHz}$ the reduction in rise and decay times is caused by the inductance in the power supply and welding leads spacing and length. ${ }^{132}$ This may be reduced by one-half by pairing the welding leads; minimizing their lengths also helps. Between 10 and $20 \mathrm{kHz}$ there may be a rapid decay of the square wave toward a saw-toothed wave depending on power supply and setup used. ${ }^{132}$ 
There are several design variations, using transistors, which shunt a portion of the welding current to obtain virtually square current pulses over the full pulse ratio and width range. All have essentially the same rate of current rise and decay characteristics.

A unique feature that is available with virtually all of the pulsed direct-current welding power supplies is a variable high frequency applied to the peak current pulse.

These are the basic types of direct-current, pulsed-current welding power supplies. Variations of these features in welding power supplies indicate that man is not lacking in ingenuity.

Each of the types of pulsed direct-current welding power supplies can be obtained with a multitude of sequencing and programming controls from the comparatively simple hot start, upslope, weld, downslope, and final current to complicated programming with numerical ${ }^{127}$ and computer ${ }^{128}$ controls for such parameters as wire feed rate, pulse rate, pulse height, and frequency and amplitude of the superimposed high frequency.

\section{c. Investigations}

The investigation of pulsed, direct-current welding power supplies involved autogenous, gas-tungsten-arc fully penetrated fillet butt-welding a thin to a thick section of maraging steel, making a simultaneous weld on each side of the thin section to the thick section. Metal for the fillets was supplied from the thick section. No evidence of undercutting of the thin section was produced. The electrodes were normal to the thick section in the direction of travel. Alternate pulsing of the two power supplies was obtained by cross-connecting the two pulsing control units. This required that the time periods of the peak and base pulses of each power supply be set at the same value. Inter-connection of the pulsing units permitted changing the time lengths of the pulses.

\section{d. Basic Findings}

- Virtually all of the basic findings listed under Direct-Current Welding Power Supplies apply to pulsed direct-current welding power supplies. 
- The smoothness of the weld surface and root depends on a combination of:

- Travel speed

- Peak and base current pulse amplitudes

- Pulse lengths

- Depth of penetration of the fusion zone depends on a combination of:

- Electrode tip shape

- Electrode diameter

- Peak pulse time

- Peak pulse current

- Base pulse time

- Base pulse current

- Average current ${ }^{129}$

- R.M.S. Current ${ }^{129}$

- Squareness of current pulse

- Rate of current rise and decay

- Shielding gas

- Travel speed

- Material

- Thickness of material

- Sufficient arc force was produced using a $2.4 \mathrm{~mm}$ (0.945. in.) diameter, $2 \%$ thoriated tungsten electrodes with $90^{\circ}$ included angle points to melt metal from the thick section to provide a fully penetrated double fillet butt weld between the thin and thick sections without over-heating the thin section.

- The depth and width depend on a combination of the following:

- Electrode tip geometry

- Electrode diameter

- Peak pulse current and time

- Base pulse current and time

- Pulsing rate

- Shielding gas

- Travel speed 
- Average current ${ }^{129}$

- R.M.S. current

- Rate of current rise and decay

- Pulsed direct-current welding power supplies are available with pulse shapes, widths, heights, pulsing frequencies, programming numerical control and computer connections to fit the most demanding requirements. In addition, superimposed variable high-frequency and current amplitude pulsing on the background and peak currents, either separately or collectively are available on many welding power supplies.

The advent of the 200 and 400 ampere transistors will greatly improve and simplify the performance of constant current and pulsed current directcurrent welding power supplies.

One investigator using Inconel Alloy 600 cold rolled and annealed sheet 0.109 inch $(2.77 \mathrm{~mm})$ thick found there is no evidence that pulsing the welding current at 2, 10, and $20 \mathrm{KHz}$ produced no observed marked advantage in the metallurgical quality or weld joint configuration. ${ }^{129}$ The audible noise generated by the arc during the high-frequency operation could be harmful to the operator and in any instance is objectionable to individuals working in the area immediately adjacent to the welding operation.

The same investigator found there is no evidence that variable highfrequency current superimposed on a low-frequency pulse mode of welding showed any improvement in the resulting metallurgical structure and geometrical configuration of the weld.

An investigator using Inconel Alloy 600 tubing with a $5 / 8 \mathrm{in} .(15.18 \mathrm{~mm})$ diameter and with a 0.080 inch $(2.03 \mathrm{~mm})$ wall explored the effects on welds with pulse period rates from 2 to $33 \mathrm{KHz}$ with and without superimposed high frequency current. Travel speed in all cases was 10 inches $(254 \mathrm{~mm})^{133}$ per minute. He found that with pulse frequencies from 2 to $22 \mathrm{KHz}$ that weld penetration and depth to width ratios of the weld fusion zones are improved.

With 2 to $22 \mathrm{KHz}$ superimposed on the peak current only, in one series of tests and on both the background and the peak pulses in the second series of tests no evidence of grain refinement was found in either case. In both 
cases, at the higher frequencies there was an improvement in the depth to width ratios of the weld fusion zones. 133 .

The prime function of the background current is to maintain an electron emission temperature on the tungsten electrode tip which will permit the high rates of current rise required for precision welding. 131 see Figure 39 , page 54.131 The current value is selected empirically to be low enough to avoid heat buildup, varying from not less than the peak pulse duration to not more than three times the peak pulse duration.

When welding sheet metal less than $0.26 \mathrm{~mm}$ ( 0.012 in.), a plasma jet is virtually required to prevent wandering of the anode spot. On very thin sheet, $0.13 \mathrm{~mm}(0.004$ in. 0$)$, it is not possible to maintain a low enough background current with low enough heat to produce a stable anode spot without melting the faying surfaces. 131

Grain refinement was obtained in fully penetrated bead on plate welds of $0.063 \mathrm{in} .(1.59 \mathrm{~mm})$ with steel plate at speeds below 2 I.P.M. $(0.42 \mathrm{~mm} / \mathrm{sec}) .134$ The grain refinement obtained in these welds is clearly due to cycling the temperature above and below the austenitizing temperature with the current pulsing. No grain refinement was obtained with Ti-11.5 Mo - $6 \mathrm{Zr}-4.55 \mathrm{Sn}$ with the same thickness of material.

\section{Alternating-Current Welding Power Supplies}

This study was started in 1956. There were no 30 to 300 ampere current single range, alternating-current welding power supplies on the market of either the manually adjusted or the remote control types. The first remotely controlled 30 to 300 ampere single range balanced sine wave current was manufactured in January 1962.135

The joints to be welded were the ends of the aluminum clad, tubular, nuclear fuel elements which were brazed with an A-13 alloy. Welding was primarily an inspection tool to uncover defects such as voids and inclusions in the braze. Requirements of the welding process were:

Good arc starting

Smooth readily inspectable weld surfaces 
Good alloying of the braze metal and the cladding into the weld A closure craterfill that was free of craters and cracks

The only power supply available that came the nearest to fulfilling all of the requirements was a dual range, moveable coil, capacitor balanced and manually adjusted unit with a compressed air operated crater filling device. A multiple range, saturable reactor type with high-frequency stabiliziation and no current balancing was studied for application of current programming and found to be totally unsatisfactory as the weld alloying was extremely poor and the weld surface was corrugated.

At this point the study was directed toward the development of a welding power supply that would fulfill all of the requirements for welding aluminum clad nuclear fuel at the Hanford Works, Richland, Washington 99352. Enough information has been gained that the following parameters were found essential for a power supply to satisfy the weld requirements:

- At least 150 volts open-circuit to produce positive arc starting and stable crater filling ${ }^{136}$

- A current wave shape approaching a square to improve alloying, surface finish, and arc starting and stability

- Balanced current waves

- A maximum time of $1 / 2$ second for the current to rise from minimum to maximum or to decay from maximum to minimum.

- A maximum current of at least 300 amperes

- A minimum current of no greater than 30 amperes

In Figure 75 is shown the range of current wave shapes investigated. They range from an unbalanced third harmonic distorted sine wave through a square wave.

\section{a. Power Supplies Studied}

i. Saturable Reactor. In Figure 76 are shown the characteristic oscillograms of an 80 volt open-circuit, high-frequency stabilized, saturable reactor controlled welding power supply. The spikes of power below the zero line in all power traces are artifacts caused by circuit components. The current is not only unbalanced but it is also distorted 

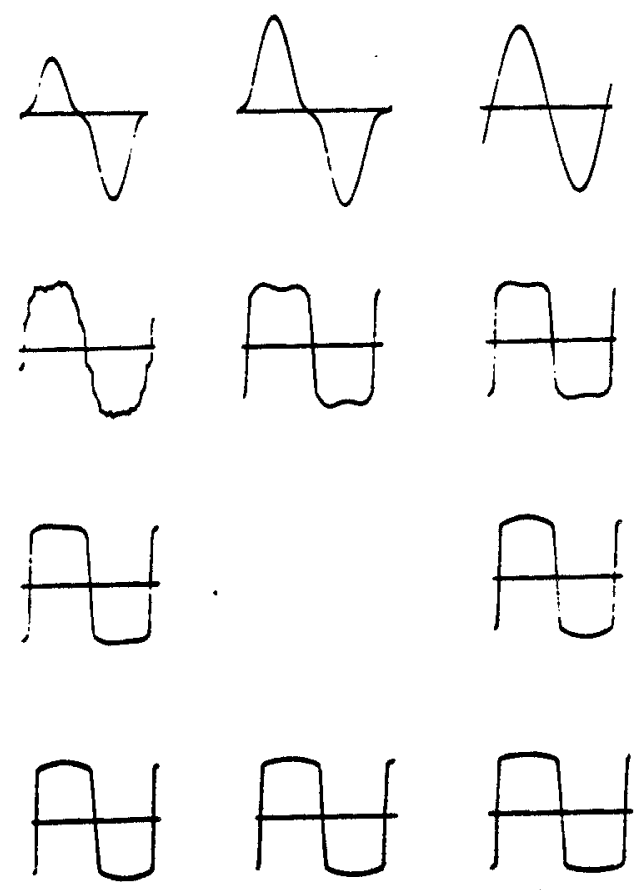

FIGURE 75 . Range of Current Wave Shapes Investigated 125

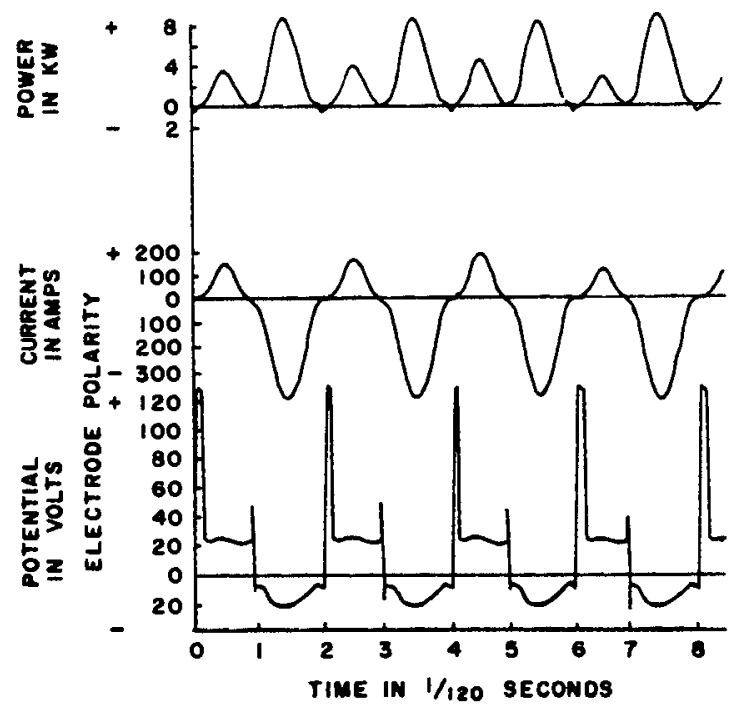

Shielding Gas 5-7 Argon-Helium Volume Ratio

FIGURE 76. Saturable Reactor 80-Volt Open Circuit on Unbalanced Current High Frequency Stabilized Third Harmonic Distorted Sine Wave 125 
with a third harmonic and has variable amplitude. Third harmonic distortion increases the time required for the polarity to reverse and restrike the arc. This is shown by the high magnitude of the restrike voltage when the electrode is positive. The result of these conditions is that virtually all of the power delivered to the weldment is when the electrode is negative. The end result is poor arc starting, very poor alloying in the weld, a rough and ragged weld, and slow welding speeds. In addition, the current range was too short to permit crater filling. Increasing the open-circuit potential to 165 volts by connecting two units in series helped to stabilize the arc but had no noticeable effect on alloying or surface roughness. Balancing the current of the 165 volt open-circuit unit with series capacitors produces the results shown in Figure $77^{125}$ The maximum pulse of power now occurs with the electrode positive. When the electrode is positive for equal currents, the arc covers a wider area than when the electrode is

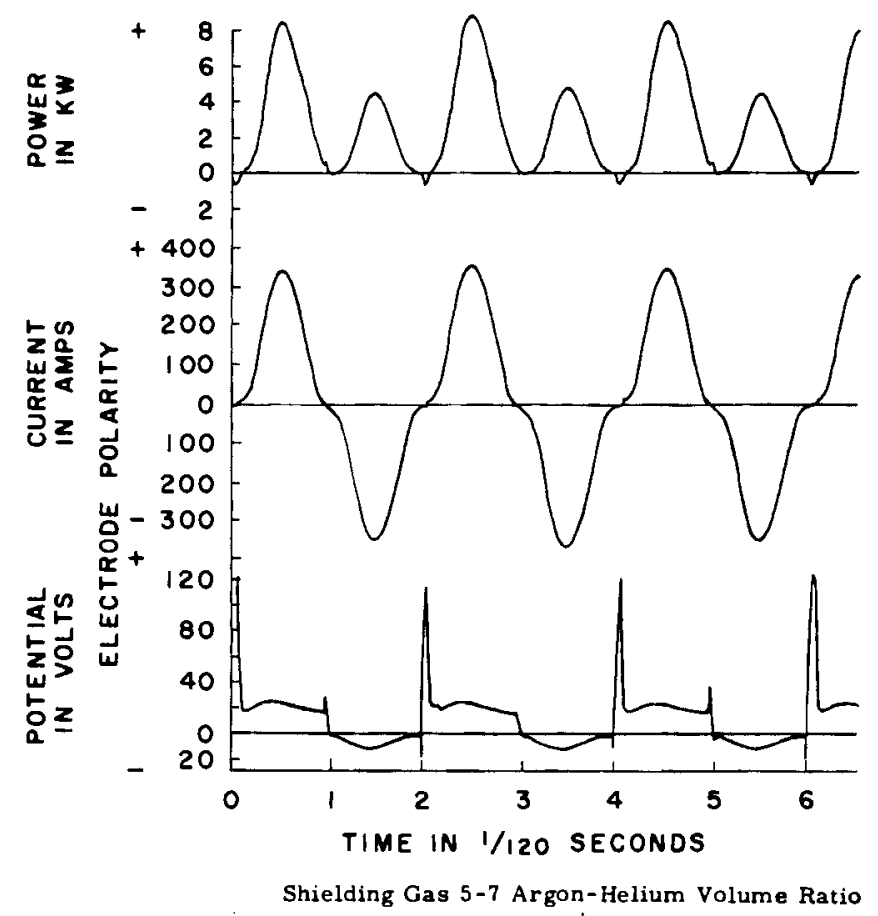

FIGURE 77. Saturable Reactor 165 Volt Open-Circuit Balanced Current No Stabilization Third Harmonic Distorted Sine Wave ${ }^{125}$ 
negative which produces a smoother weld surface. The surface produced was acceptable, and crater filling was adequate, but there was virtually no improvement in alloying.

ii. Moveable Coil. Transformer. Figure $78^{125}$ shows some characteristic welding arc oscillograms of a sine wave current welding power supply having unbalanced current and 80 volts open-circuit without high-frequency stabilization. There is not enough open-circuit voltage to re-ignite the arc when the electrode is positive. In effect, it is essentially a rectifier. The addition of series capacitors, Figure 79, 125 improves the arc starting and stabilizes the arc at welding value of current. However, as the current decays, a point is reached at which the re-strike voltage with the tungsten electrode positive is no longer of sufficient magnitude, and the arc does not re-strike. The arc re-strikes when the tungsten electrode becomes negative and places a second charge on the series balancing capacitors. When the tungsten electrode again becomes positive, the additional charge on the capacitors raises the re-strike voltage to the point that the arc will re-strike.

Increasing the open-circuit potential to 150 volts by tap changing without capacitor balancing produces positive arc starting, a stable arc, and full crater filling (Figure 80 ). 125 The addition of series capacitors balances the current and produces a smoother weld surface. In Figure $81^{125}$ are shown the characteristic voltage, current, and power oscillograms of a balanced sine wave of current with 150 volts open-circuit. Re-strike voltages are adequate and the valleys between the power pulses have narrowed, producing improved weld alloying.

iii. Selin Poly Reactor. Figure $82^{125}$ shows the welding arc oscillograms for a balanced current whose instantaneous values are the cube root of the instantaneous values of sine wave. $137,138,139$ It was produced with a Selin reactor which is a group of saturable reactors connected in series, each one conducting at a later time in the half cycle of voltage. This permits the tailoring of a wave shape and frequency to fit the needs. The opencircuit potential of the unit was 165 volts. In the oscillogram for the arc voltage, the height of the re-strike spike with the tungsten electrode positive indicates that there is adequate re-strike voltage. 


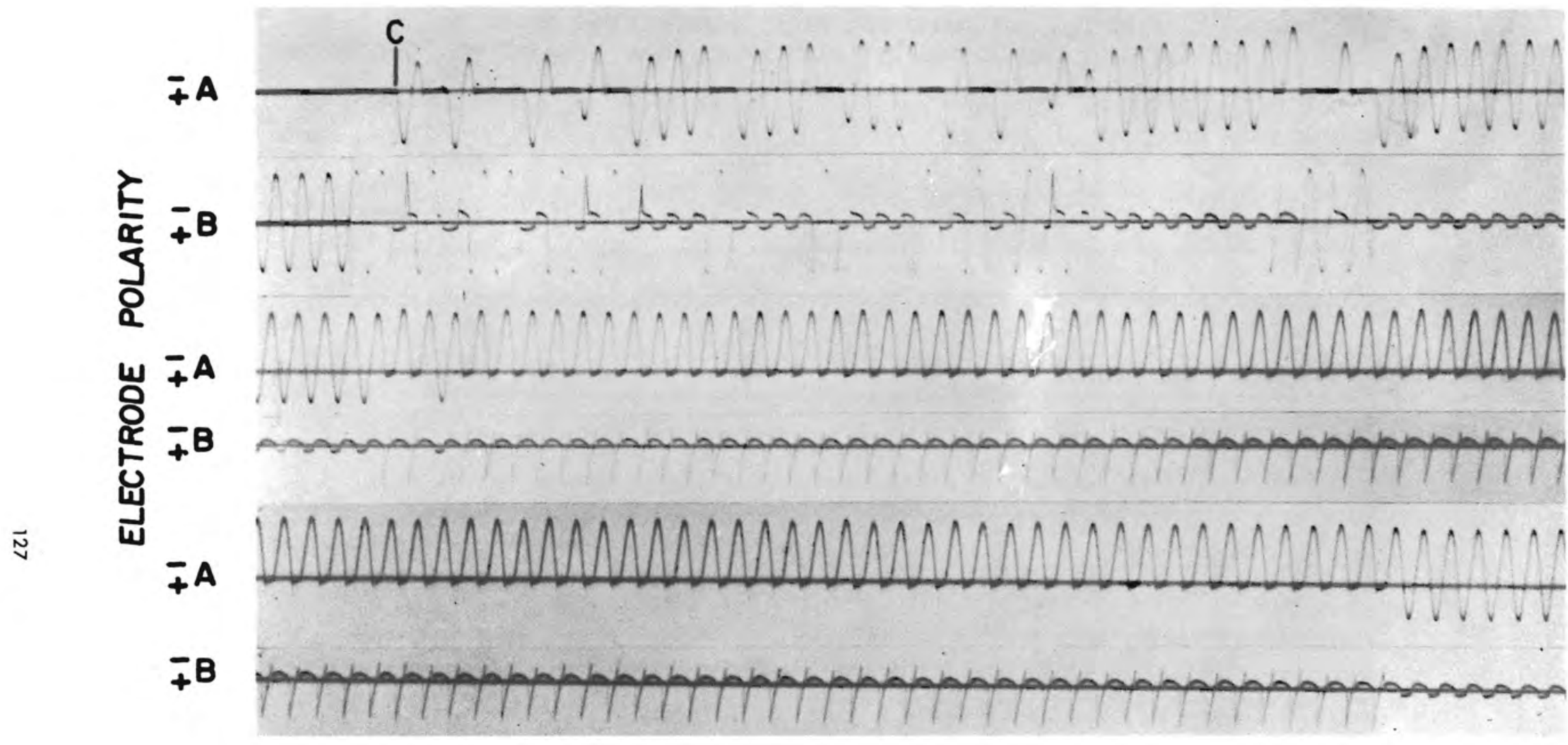

\footnotetext{
Argon Shielding Gas

A - Arc Current 135 A Rms

B - Arc Voltage $135 \mathrm{~V}$ Rms

C - Arc Starting Point
}

FIGURE 78. Moving Coil Transformer Oscillograms of Arc Starting Sequences for 100 Volts 0penOpen-Circuit Sine Wave Current With No Stabilization or Balancing 24 
C

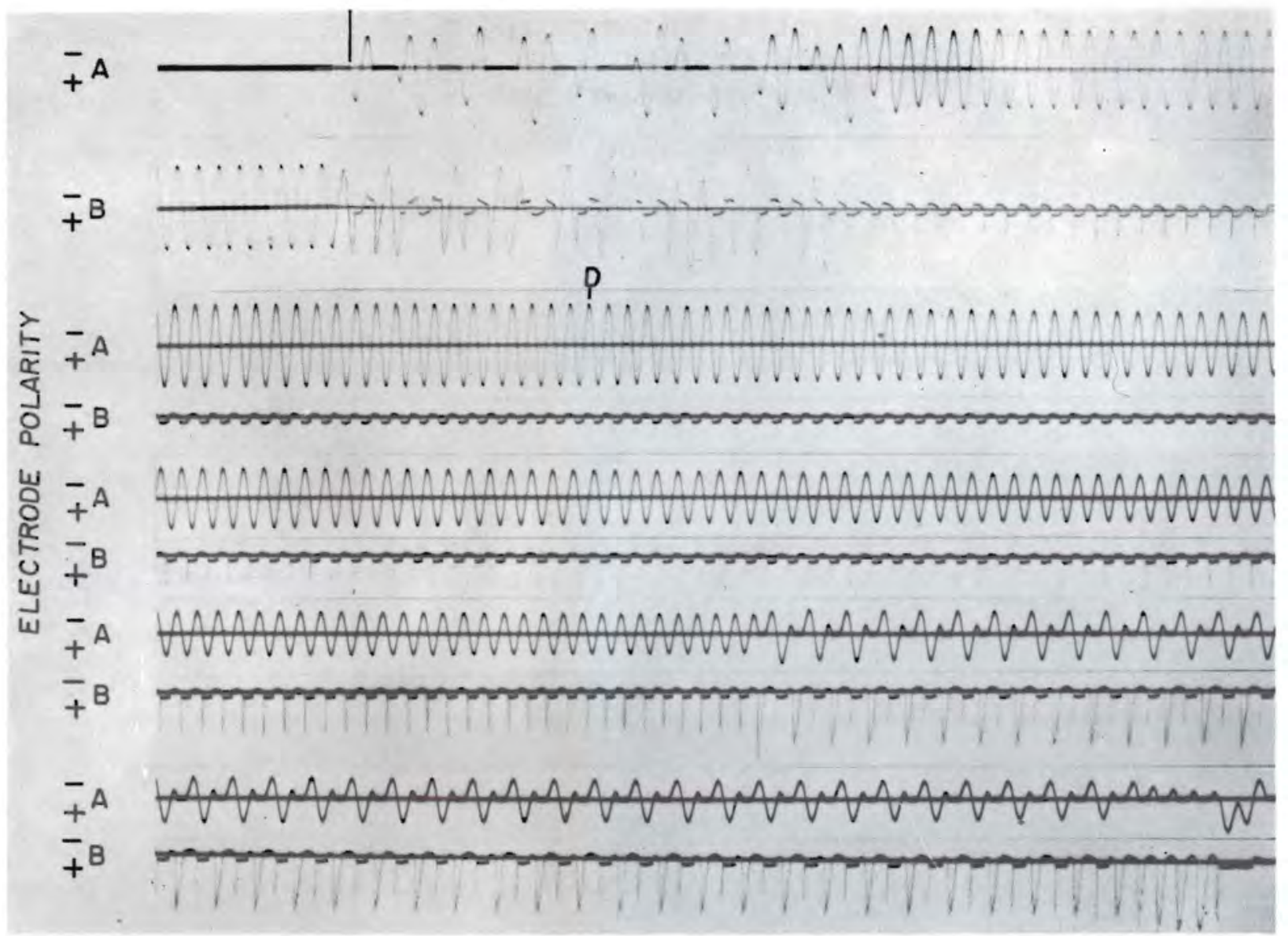

A - Arc Current 135 A Rms

B - Arc Voltage 135 Volts Rms
C : Arc Starting Point

D - Crater Filling Start

FIGURE 79. Moving Coil Transformer Oscillograms of Arc Starting, Welding, and Crater Filling Sequences for 100 Volt

Open-Circuit Balanced Sine Wave Current Arc, No Stabilization 124 


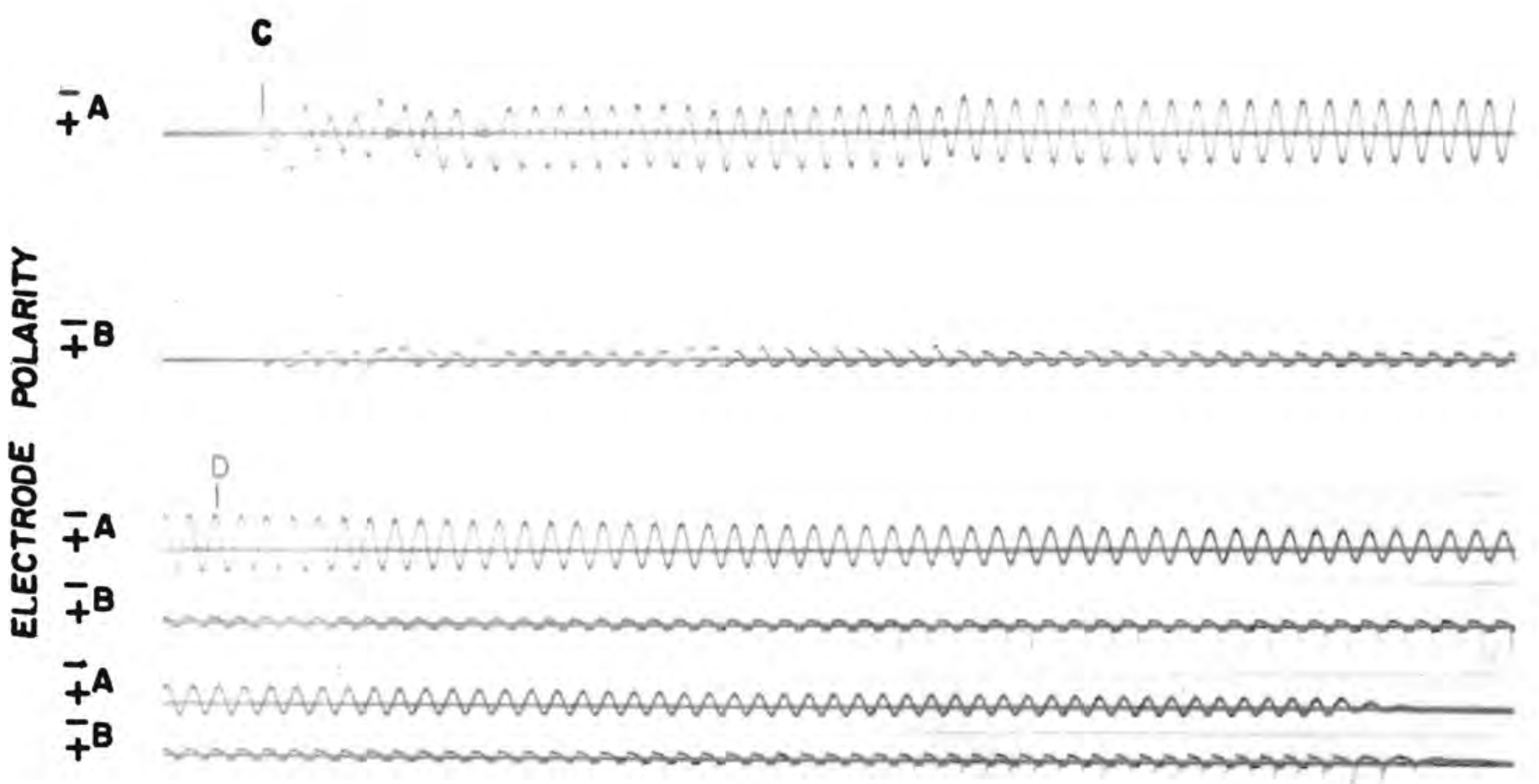

$$
\begin{aligned}
& \text { A - Arc Current } 135 \text { A Rms } \\
& \text { B - Arc Voltage } 135 \text { V Rms } \\
& \text { C - Arc Starting Point } \\
& \text { D - Crater Filling Start } \\
& \text { Argon Shielding Gas }
\end{aligned}
$$

FIGURE 80. Moving Coil Transformer Oscillograms of Arc Starting, Welding, and Crater Filling Sequences for 150 Volt

Open-Circuit Sine Wave With No Stabilization or Balancing 124 


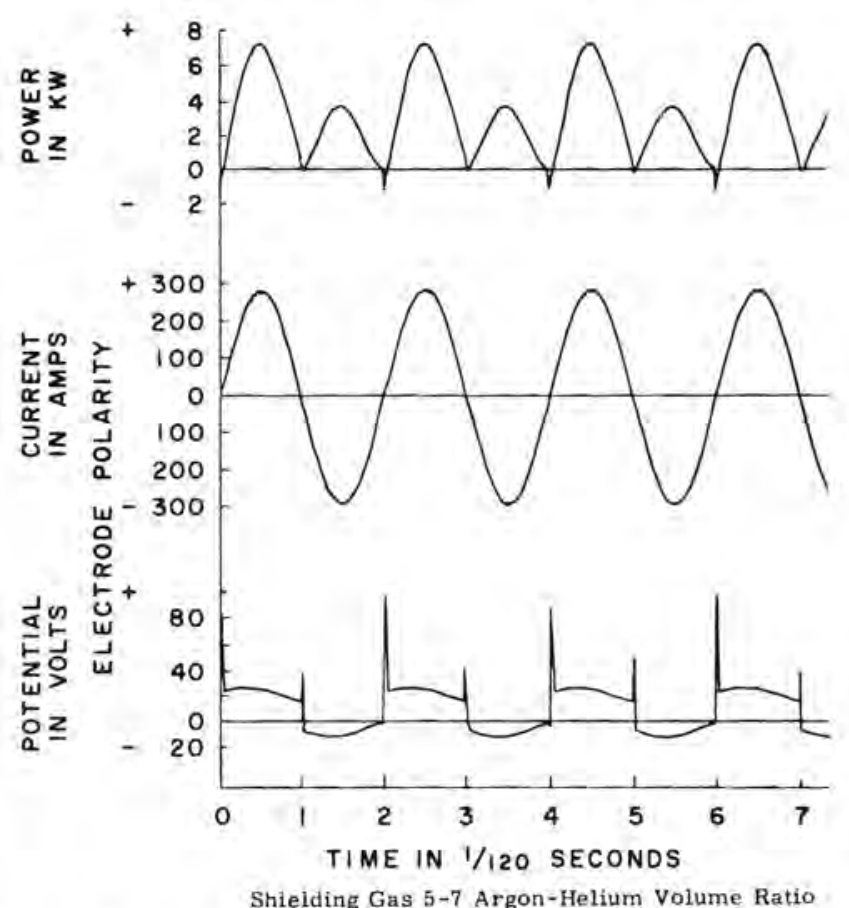

FIGURE 81. Moving Coil Transformer Oscillograms of 150 Volt Open-Circuit Balanced Sine Wave Arc, No Stabilization 125

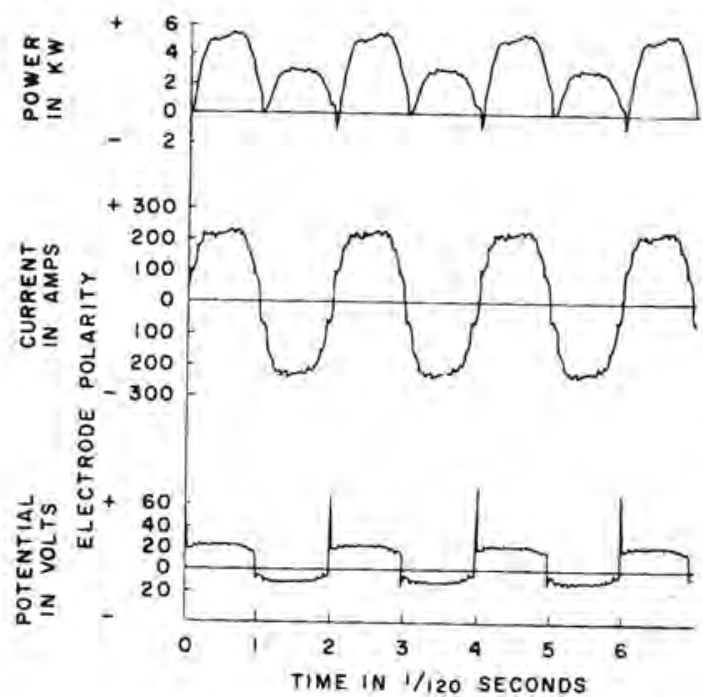

Shielding Gas 5-7 Argon-Helium Volume Ratio

FIGURE 82. Selin Saturable Reactor Oscillograms of 165 Volts Open-Circuit,Balanced Cube Root Sine Wave Arc, No StabiTization 125 
The lack of symmetry in the top of the current wave is caused by arc hysteresis. This also produces the variation in symmetry of the sides of the current waves. The gaps in the power have been filled some more, producing a more uniform temperature in the weld, better alloying, and smoother weld surfaces.

iv. Square Wave Saturable Reactor. In Figures 83 and 84 are shown two types of square current waves. The first one was produced with a saturable reactor having a large inductance and third harmonic filter in series with the saturating control circuit. The second one has just a very large inductance in series with the saturating control circuit. $140,141,142,143$ Examination of the re-strike voltage shows that it is very much smaller than that for the other types of current waves indicating that the polarity reverses very fast and that the arc re-strikes instantaneously. On the original oscillograms there is a very small discontinuity visible in the current trace where the arc re-strikes when the tungsten electrode is positive. Examination of the power pulses shows that the valley is almost completely filled in. This maintains a more constant temperature in the weld and increases the period of agitation of the puddle with the arc, producing the best alloying and a very smooth weld surface. Flatness of the top of the wave is also detectable in the alloying--the flatter the top of the wave, the better the alloying.

Since the time for the polarity to reverse with square current waves approaches zero, the amount of open-circuit voltage to re-strike the arc and to supply adequate extinguishing voltage should be minimized. A series of tests was performed to determine the relationship between argon and argon-helium mixtures, welding speed, and open-circuit voltage without high-frequency stabilization. Stability was determined by slowly decaying the welding current manually until the arc changes from thermionic emission to cold cathode discharge, then raising the current at approximately the same speed. If the arc did not go out or become unstable, it was considered that there was adequate open-circuit voltage for arc stability. The maximum current was 300 amperes, the minimum approximately 30 amperes where the arc discharge mode transition took place. A (5/32 inch) $4 \mathrm{~mm}$ zirconated tungsten 


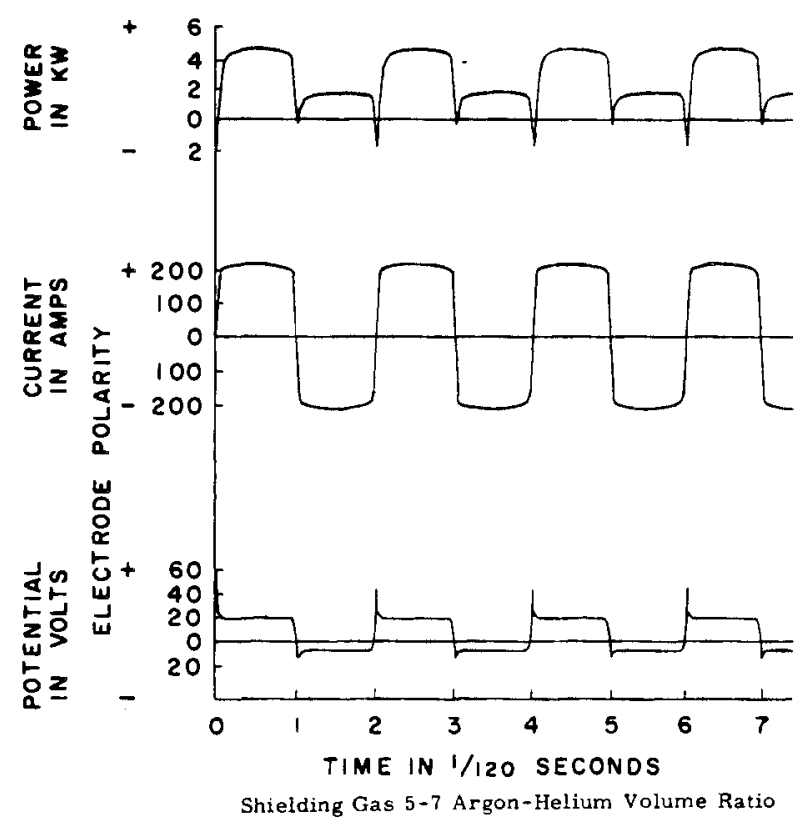

FIGURE 83. Weed Saturable Core Reactor With Fiiter. Oscillograms of One Type of Square Wave, 165 Vol $\$ \xi_{5}$
Open-Circuit, Balanced Current, No Stabilization

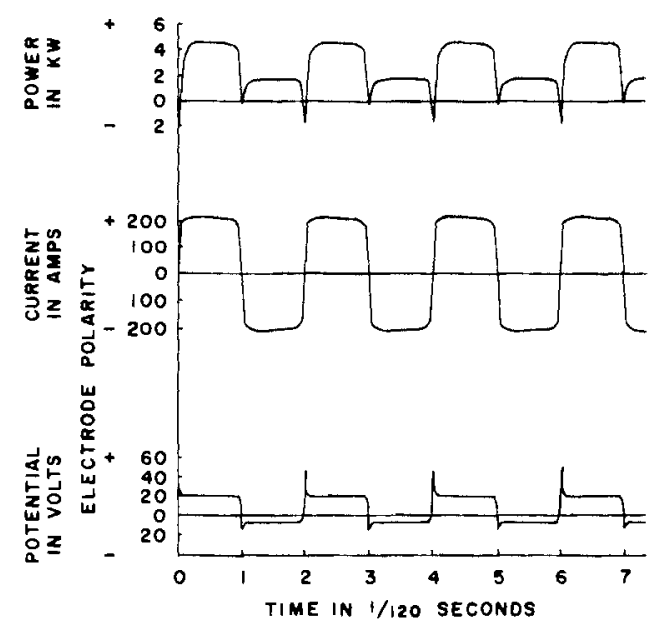

Shielding Gas 5-7 Argon-Helium Volume Ratio

FIGURE 84. Weed Saturable Reactor With Inductance.

Oscillograms of Arc of Another Type of Scuare Wave, 165 Volts Open-Circuit, Balanced Current, No Stabilization. Shielding Gas 5-7 Argon-Helium Volume Ratio 
electrode was used. From the results of the tests shown in Table 4 it was found that 130 volts open-circuit would produce arc stability with a 1 volume part argon, 3 volume parts helium shielding gas mixture. The test showed that stability was dependent on there being enough open-circuit voltage to establish a cold cathode discharge when the polarity reversed and the aluminum became negative.

v. Pulsed Square Wave Alternating-Current. The final result of the work on the development of the alternating-current welding power supplies was the idea that if there was some way of providing the cathodic cleaning necessary to produce a high quality weld with a smooth surface, then the welcing could be done with direct-current electrode negative. This was the birth of pulsed square wave alternating-current welding in which a short square electrode positive pulse of current is used to cathodically etch the surface of the weld area followed by a long electrode negative pulse of the same amplitude to do the welding. ${ }^{144,145}$ In Figure $85^{146}$ are shown oscillograms

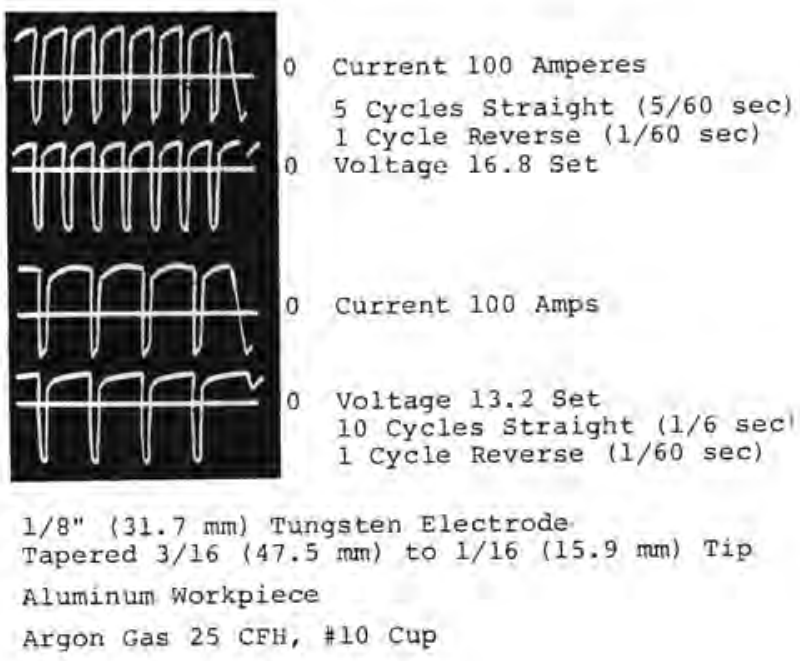

FIGURE 85. Pulsed Square Wave Alternating-Current ${ }^{\top 46}$ 
of current and voltage of the pulsed square wave alternating-current arc. The electrode positive pulse is one-half cycle and the electrode negative pulse is ten half cycles. Other combinations of time periods and relative pulse heights have been found useful.

Pulsed square wave alternating-current controls which operate in conjunction with premium grade direct-current, inert-gas shielded tungsten-arc welding power supplies are available in continuously adjustable, single range welding power supplies to $600 \mathrm{~A}$. Pulse lengths of the electrode negative arc are from 1 cycle to 20 cycles in 1 cycle steps and the electrode positive is adjustable from $1 / 2$ to 10 cycles in half cycle steps. The minimum current is 10 amperes. The time of rise of current from minimum to maximum and to decay from maximum to minimum is in the order of 6 to 7 cycles, which permits very fast programming. These times are based on using a direct-current power supply that has equally fast response times. This system produces very high quality welds with fine surface finish.

A pulsed square wave alternating-current welding power supply is available which has independent time control of each half cycle and requires no capacitors to produce welding current symmetry when gas tungsten arc welding a1uminum. 147

In an extensive evaluation of the autogenous automatic butt welding of one inch, Schedule 5, alloy 3003 aluminum pipe using an orbital head, the following parameters were developed: ${ }^{148 *}$

- Alternating-current having equal time periods and amplitudes for both the electrode negative and electrode positive currents (square waves) produced the fewest porosity, stringers, and lack of root fusion. Internal bead surface smoothness was also best as were concentricity and fa11-through.

- There was no evidence that an internal purge of inert gas was of any advantage.

*For a complete detailed description of the work, see Supplemental References 27 and 28. 
- It was difficult to see any advantage to the pulsing of the straight polarity pulse of current.

- The best overall internal and external weld quality produced with a chopped sine wave was obtained with $20 \mathrm{cfh}$ ( $575 \mathrm{Lh}$ ) helium and $7 \mathrm{cfh}$ (195 Lh) argon, pulsed straight polarity average current reading of 37 amperes with a 16 percent difference between the high and low pulse currents and a reverse polarity reading of 21 amperes average reading. The rotational speed was 7 RPM.

- Two wave shapes were selected as producing the required weld qualities.

A virtually low square wave of equal positive and negative amplitudes and time periods.

A chopped sine wave such as produced with thyristor or a grid controlled tube. The two pulses were of equal time periods and had equivalent wave shapes. Due to the chopping there is a period at the start of each pulse in which no conduction takes place.

vi. Sine Wave Saturable Reactor. A sine wave alternating-current power supply is available. The wave shape is produced with a circuit similar to the Weed ${ }^{140}$ circuit used to produce the square current waves. It has two wide continuously adjustable current ranges which may be used either with or without series capacitor current balancing. Shown in Figure $86^{149}$ are the oscillograms of the balanced current waves of the low and high current range. An open-circuit potential of 150 volts is available for arc starting and re-striking. Without an arc the maximum electrode to ground potential is 80 volts.

\section{b. Basic Findings}

The basic findings of the study of alternating-current welding power supplies are:

- Arc starting, arc, half-cycle restriking, half-cycle extinguishing, and crater filling stability are determined by the open-circuit voltage for a particular wave shape, welding speed, and weldment material.

- The major portion of the cathodic etching, or cleaning, takes place while the current is rising. 

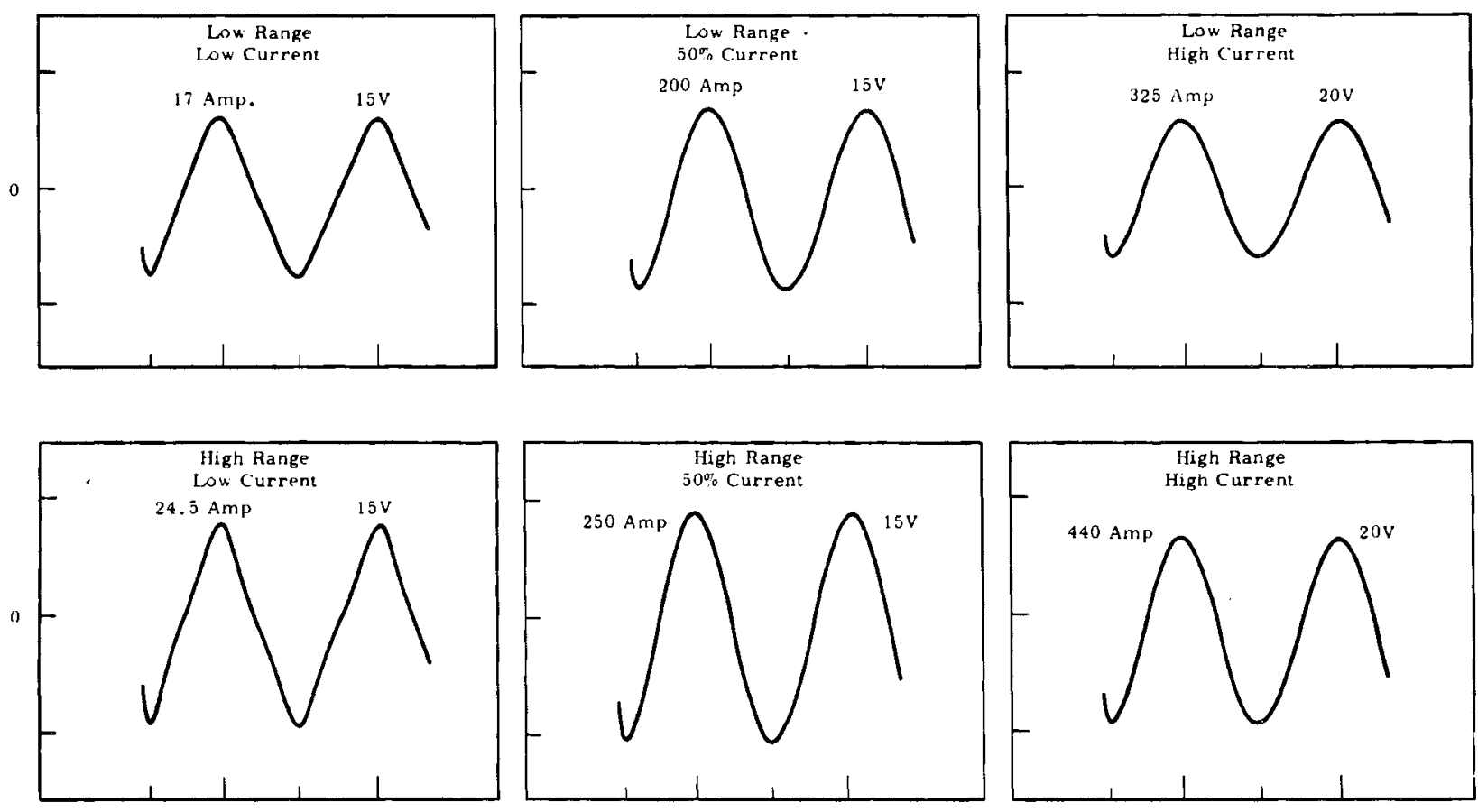

FIGURE 86. Wide Ranges, Balanced Sine Wave furrent
Alternating-Current Power Supply 149

- The addition of resistance into the welding current carrying circuit during arc starting limits starting transients that blow craters in the workpiece.

- As the current wave shape changes from a narrow high peaked third harmonic distorted sine wave toward a square wave, the arc starting, half-cycle re-strike, half-cycle extinguishing, and crater filling improve, and less open-circuit voltage is required.

- Weld alloying, quality, and surface finish improve as the current wave shape changes toward a square wave.

- Welding speed increases as the current wave shape changes toward a square wave.

- As the welding speed increases, the open-circuit voltage must be increased to maintain arc stability.

- Wide single and double range welding power supplies are available with fine programming. 
- The current wave shapes of alternating-current welding power supplies can be tailored to the work requirements.

- Balanced square current waves permit the welding of aluminum at 80 volts open-circuit without high frequency or other stabilization when welding with argon shielding gas.

- Single range pulsed square wave alternating-current welding power supplies are available in precision and general purpose welding models. Electrode negative and electrode positive pulse times and pulse amplitudes are independently adjustable on some designs. 



\section{PROCUREMENT}

Experience gained in the procurement of the welding power supplies used in this work has developed the following suggestions for procuring precision welding power supplies. The job needs to be analyzed for:

\section{A. CONSTANT CURRENT POWER SUPPLIES}

- Allowable current ripple for direct-current welding power supplies allowing for change in ripple as current changes.

- Allowable wave shape for alternating-current welding power supplies allowing for the change in shape as the current changes.

- Open-circuit voltage rquired

- Arc starting

- Arc starting and welding

- Variable open-circuit voltage

- Arc starting and welding voltage ranges

- Maximum allowable current rise and decay times

- Current upslope and downslope

- Linear or exponential slope

- Slope time ranges and precision

- Arc starting characteristics required:

- High frequency starting

- Pulse starting

- Logic Controls

- Number of controls required

- Precision of each control

- Range of each control

- Constant current control precision as affected by:

- Line voltage variation

- Welding current programming

- Drift with time and temperature

- Response time 
- Programming controls

- Sequential

- Numerical

- Computer

- Hot start control range

B. PULSED CURRENT POWER SUPPLIES

- General. If current pulsing is required, check which of the following functions is needed and the precision and range of each:

- Pulsing frequency, adjustable range

- High pulse width, adjustable range

- Low pulse width, adjustable range

- Pulsing on upslope

- Pulsing on downslope

- Pulsing on hot start

- Pulsing on initial current

- Pulsing on final current

- Superimposed high-frequency pulsing and frequency range

- Shape of current rise curve minimum to maximum

- Maximum time for current to rise from minimum to maximum currents

- Shape of current decay curve maximum to minimum

- Maximum time for current to decay from maximum to minimum

- Hot start

- Adjustable current range

- Adjustable time range

\section{SPECIFICATIONS}

- Write a tight comprehensive specification for the power supply needed including a checkout at the manufacturer's plant.

- Make a complete checkout of each unit at the manufacturer's plant in accordance with the specification. The points to be checked out are spelled out in detail in the contract as to how and with what shall be used in the checkout. 
- Use precision instrumentation. Direct writing recorders and "Simpson" type test equipment do not have the required precision and response times. 


\section{MAINTENANCE}

Experience gained in the operation of precision power supplies and welding systems indicates that the following points should be checked regularly, the frequency depending on the amount of use of the equipment:

- Ripple and wave shape of the welding current with a cathode ray oscilloscope

- Current, voltage, and travel readouts with precision instruments (not "Simpsons" or equivalents)

- All timing devices

- Cooling air inlet filters and cooling air outlets

- Al1 heavy current manual and magnetic switches

- All welding and ground current connections 


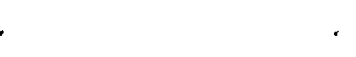




\section{SUMMARY}

Arc welding is a metal melting and casting process in which the metal to be melted is either the base metal, supplied as an auxiliary filler metal, or a consumable electrode. The heat to melt the metal is supplied by the arc whose heat is determined by the arc voltage and current. Lengthening the arc increases the arc power for the same current, but penetration decreases and weld width increases. Power generated in a welding arc is almost a linear function of current since the arc voltage remains almost constant in the welding range for a particular electrode size as the welding current changes. Characteristics of the arc are determined by electrode size, tip shape and composition, current, shielding gas, shielding gas pressure and filler, and base metal compositions. As the current increases, a point is reached at which the electromagnetic forces surrounding the arc cause a sudden constriction of the arc producing deeper penetration. Slowly raising a current in helium and tungsten arcs, a point is reached at which there is a sudden rise in voltage as the arcs constrict. Decreasing the current to just below the point of voltage rise, a point is reached at which the voltage suddenly drops as the arc expands.

Some of the elements form slags on the surface of the weld puddle limiting heat transfer and penetration. Essentially all elements in and on the electrode, filler, and base metals enter the arc and affect the voltage, shape, and, thus, the penetrating properties of the arc. Minute quantities of some materials grossly affect the arc and its voltage while rather large quantities of other materials have a very small effect. This effect is determined by the ease of electron emission, the vapor pressure of the material at electrode, and arc column temperature and pressure.

Welding with the gas tungsten arc in atmospheres of argon, helium, and their mixtures has been investigated to 40 bars $\left(400 \mathrm{~N} / \mathrm{cm}^{2}\right)$ and techniques developed for welding Zircaloy clad nuclear fuel pressurized to 40 bars $\left(400 \mathrm{~N} / \mathrm{cm}^{2}\right)$.

Gas shielded metal arc and shielded metal arc welding have been investigated and welding techniques developed to 32 bars $\left(320 \mathrm{~N} / \mathrm{cm}^{2}\right)$ for underwater welding. 
Equations have been developed for calculating the plasma energy transfer in gas shielded welding arcs from the cathode and into the weld fusion zone. The equations have been developed to cover the major sections of the arc. Regions covered are:

- Energy interchange between the cathode and column

- Plasma energy transfer in the column

- Plasma energy transfer to the anode without current flow and without melting

- Plasma energy transfer to the anode with current flow and without melting

- Plasma energy transfer to the anode with current flow and with melting The welding arc receives its power/energy for melting the metal from the welding power supply. The efficiency and quality of the weld produced, assuming that the base metal-filler metal-shielding gas system is adequate, is determined by the characteristics of the current and voltage being delivered by the welding power supply. Ripple of direct-current varying from a constant numerical value such as a battery, depending on the amount of ripple, will affect weld width, interior quality, penetration, and surface roughness. As the current ripple increases, the open-circuit voltage must increase to maintain the arc at the deep valleys in the current.

The open-circuit voltage required to produce a stable arc is determined by the ratio of the depth of the current valley to the residual current and the times-width of the valley. This applies to both alternating- and direct-currents.

As the alternating-current wave changes from a square wave through a sine wave to a narrow high-peaked wave, alternating-current produces a rougher weld surface and requires higher open-circuit voltage and/or stabilization. This includes the effect of rectification that occurs with reversal of alternating-current when the electron emission characteristics of the tungsten electrode and the base metal arc are not equal. Techniques are avaialable for optimizing the amount of rectification for a particular requirement. 
Pulsing of the welding current between a high value and a low value produces very beneficial effects when making root passes or joining thick and thin sections. The high current pulse produces fusion and the low current pulse maintains the arc while solidification and heat runout take place.

No matter how simple or sophisticated the required weldment may be there are arc welding power supplies and programmed systems available that will produce weld quality to satisfy the requirements. At one end of the spectrum is the simple tapped winding transformer for stick electrode welding and on the other end computer programmed, multi-axis arc welding systems for aerospace and nuclear systems.

Constant-current direct-current power supplies are available with single ranges as great as 50 to 1 with current ripple over the full current range equivalent to a motor generator set. Their current rise and decay times from minimum to maximum and maximum to minimum current for the finest units are on the order of a few microseconds.

Pulsed-current, direct-current welding power supplies are available all the way from ones for a very ordinary job to ones with all of the sophistication of constant-current, direct-current welding power supplies. In addition, the pulsing options available appear to exceed the present needs of the welding industry. Besides pulsing the welding current, some superimpose higher frequencies on the high and low current pulses, selectively or concurrently.

Alternating-current welding power supplies are available with single continuously adjustable ranges on the order of 10 to 1 up to 60 to 1 , depending on the particular wave shape. The current wave shapes vary from a third harmonic distorted sine wave through a chopped sine wave to a square wave to pulsed square waves. In pulsed square waves the heights of the pulses may be independently adjusted for both polarities. The time period for the electrode negative pulse may be adjusted from 1 to 20 cycles and the length of the electrode positive pulses from $1 / 2$ to 10 cycles for one power supply. 
Open-circuit voltages up to at least 150 volts may be obtained with both direct and alternating-current welding power supplies for mechanized welding. Pressurized welding requires open-circuit voltage as high as 400 volts. These high open-circuit voltages are lethal and are not allowed for hand welding unless approved circuitry is used that limits the opencircuit voltage to 80 volts or less when the arc is extinguished.

Arc starting with helium for six current pulses per cycle directcurrent welding power supplies has been improved with higher open-circuit voltages, higher current rates of rise times, and lower current ripples.

Twelve current pulses per cycle rectifier type, direct-current constant current welding power supplies permit excellent arc starting on aluminum at any point on the current range with pure helium shielding gas using thoriated tungsten electrodes and high-frequency arc starting. Tests run with a $200 \mathrm{~A}$, 12 current pulses per cycle magnetic amplifier welding power supply disclosed, in addition, the following:

- Stable operation at any point on the current range

- Higher rates of rise and decay of the welding current

- Higher welding speeds

- Smooth welds

- Better weld quality 


\section{REFERENCES}

1. Rich, J.A., "Resistance Heating in the Arc Cathode Spot Zone," Jn1. App. Phys., 32 1030, (1961).

2. Cobine, J.D. and Ga1lagher, C.J., "Current Density of the Arc Cathode Spot," The Phys. Rev., 74, 1528 (1948).

3. vonEnge1, A., Ionized Gases. Oxford University Press, 275, (1965).

4. Cobine, J.D., Gaseous Conductors, Dover Publications, 300, (1958).

5. Goldman, K., "Electric Arcs in Argon: Heat Distribution," Brit. Weld Jn1., 10, 347, (1965).

6. Goldman, K. and White, E.S., "Effect of Anode Material on Arc Mechanism," Brit. Weld. Un1., 12, 430, (1965).

7. Cobine, J.D. and Gallagher, C.J., "Current Density of the Arc Cathode Spot," The Physical Review., 74, 1524, (1948).

8. Private communication with J.A. Rich, July 27, 1961.

9. Rich, J.A., "Resistance Heating in the Arc Cathode Spot Zone," Jn1. App. Phys., 32, 1027, (1961).

10. Op. cit., 1030

11. LoC. cit.

12. Cobine, J.D., "The Nature of the Arc," WRC Bulletin 2, 3, (1949).

13. Op. cit., p. 5

14. LoC. cit.

15. Goldman, K. and White, E.S., "Effect of Anode Material on Arc Mechanism," Brit. Weld. 3n1., 12, 430-434, (1965).

16. Cobine, J.D., Gaseous Conductors, Dover Publications, 299-302, (1958).

17. Op. cit., 326-327.

18. Cobine, J.D. and Gallagher, C.J., "New Electrodes for Stabilizing Inert-Gas Welding Arcs," Trans. AIEE, 70, 805, (1957).

19. Morton, H.S. and Gage, R.M., "Arc Properties in the Five Rare Gases," Symposium on Arcs in Inert Atmospheres and Vacuum, John Wiley \& Sons, 10-11, (1956).

20. Skolnick, Merri11, and Jones, T.B., "Characteristics of the High Current Tungsten Arc in Argon, Helium, and Their Mixtures," Inl. App. Phys., 23, 648, (1952).

21. Op. cit., p. 649

22. Morton, H.S. and Gage, R.M., "Arc Properties in the Five Rare Gases," Sympsoium on Arcs in Inert Atmospheres and Vacuum, John Wiley \& Sons, Fig. 5., (1956). 
23. Cobine, J.D., "The Nature of the Arc," WRC Bulletin 2, p. 3, April 1949.

24. Savage, W.F., Strunck, S.S., and Ishikawa, Y., "The Effect of Electrode Geometry in Gas Tungsten-Arc Welding," Weld. Jn1., 44, 494s, (1965).

25. Correy, Thomas B., "Effect of Gas Pressures on Etching and Surface Roughness of Cathodically Etched Metal Surfaces," Physics of the Welding Arc. London: The Institute of Welding, pp. 224-233, (1966).

26. Feldman, J.M. and Williams, E.M., "Electrode Sputtering in High Pressure Electric Discharge," App. Scien. Res., Sec B, 16, (1963).

27. Wolsky, S.P., "Sputtering Mechanisms," Transactions of the Tenth National Vacuum Symposium, Part II, Thin Film Technology, Section I, Invited Papers, Sputtering, (1953-1963).

28. Pattee, H.A., Anno, J.H., and Randall, M.D., "Theoretical and Experimental Study of Cathodic Cleaning with Plasma Arc," Weld. Jnl., Weld. Res. Sup., 47, 183-s, (1968).

29. Rich, J.A., "Resistance Heating in the Arc Cathode Spot Zone," Jn1. App. Phys., 32, 1030, (1961).

30. Rich, J.A., Private Communication, July 27, 1961.

31. Almar-Ness, A., "Introduction to Arc Welding," Document IIS/IIW - 129 64, Prepared by Commission XIV, International Institute of Welding, American Welding Society, Miami, Florida, p. 124, (1964).

32. Cobine, J.D., Gaseous Conductors, Dover Publications, p. 302, (1958).

33. Rich, J.A., "Resistance Heating in the Cathode Spot Zone," Inl App. Phys., 32, 1028-1029, (1961).

34. Correy, Thomas B., "Waveshape Effect on Alloying and Arc Stability of A-C Tungsten Inert-Arc Welding," IEEE Applications and Industry 56, 172, (1961).

35. Cobine, J.D., Gaseous Conductors, Dover Publications, 302, (1958).

36. Ludwig, H.C., "Plasma-Energy Transfer in Gas-Shielded Welding Arcs," Weld. Jn]. 38, 299s, (1959).

37. Cobine, J.D., "The Nature of the Arc," WRC Bulletin 2, p. 1, April 1949.

38. vonEnge1, A., Ionized Gases, Oxford University Press, p. 260, (1965).

39. Cobine, J.D., Gaseous Conductors, Dover Publications, 343, (1958).

40. Loc. cit. 343

41. Morton, H.S. and Gage, R.M., "Arc Properties in the Five Rare Gases," Symposium on Arcs in Inert Atmospheres and Vacuum, John Wiley \& Sons, Fig. 5, 13, (1956).

42. Goldman, K., "Electric Arcs in Argon: Volt-Amp and Volt-Arc Gap Characteristics, "Physics of the Welding Arc. London: The Institute of Welding, 17-22, (1966).

43. Goldman, K. and White, E.S., "Effect of Anode Material on Arc Mechanism," Brit. Weld. Un1., 12, 432, (1965). 
44. Goldman, K., "Properties of the Argon Arc with Thoriated Tungsten Cathode," Brit. Weld. Un1., 10, 517-523, (1963).

45. Goldman, K., "Electric Arcs in Argon: Volt-Ampere and Volt-Arc Gap Characteristics," Physics of the Welding Arc, A Symposium, London: The Institute of Welding, Figure 3, (1962).

46. Nestor, O.H., "Heat Intensity and Current Density Distribution at the Anode of High Current, Inert Gas Arcs," Jn1. App. Phys., 33, 1641-1646, (1962).

47. Glickstein, S.S., "Temperature Measurements in a Free Burning Arc," Weld. Un1., Weld. Res. Supp. 55, 226s-227s, (1976).

48. Suits, C.G., "The Determination of Arc Temperature From Sound Velocity Measurements," Physics, 6, 195, Part I, (1935).

49. Skornyakov, G.P., "The Asymmetry of the Voltage in a Direct-Current Arc," Izvest. Akad. Nauk. SSSR, Otdel, Tekh. Nauk. No. 5, 140-143, (1956).

50. Glickstein, S.S. and Yeniscavich, W., "A Review of Minor Element Effects on the Welding Arc and Weld Penetration," 1-18, Welding Research Council Bulletin No. 226, May 1977.

51. Steenbeck, M., Physikolosche Zeitschrift, 33, (1932).

52. Almar-Ness, A., "Introduction to Arc Welding," IIS/IIW - 129-64, Prepared by Commission XIV, International Institute of WeIding, American Welding Society, Miami, Florida, 126, (1964).

53. Loc. cit.

54. Goldman, K., "Effect of Anode Material on Arc Mechanism," Brit. Weld. Un1. 9, 430-434, (1965).

55. Spiller, K.R., and MacGregor, G.J., "Effect of Electrode Vertex Angle on Fused Weld Geometry in TIG-Welding," Proceedings of the Conference on Advances in Welding, Apri1 14-16, 1970, The Welding Institute, Abington Ha11, Cambridge, England, Session III, Paper 9, 83.

56. "How to Avoid Inclusions in TIG Welds," Met. Fab. 41, 50-51, (1973).

57. Savage, W.F., Strunck, S.S., and Ishikawa, Y., "The Effect of Electrode Geometry in Gas Tungsten-Arc Welding," Weld. In1.2 44, Figs. 3,7, (1965).

58. Lander, Harvey and Thomson, Donald S., "PIGME, A New Automatic Inert Arc Welding Process," Amer. Soc. of Tool and Mfg. Engrs., Paper No. AD67-195, 1-11.

59. Sparagen, W., and Lengyel, Bela, "Physics of the Arc and the Transfer of Metal in Arc Welding," Weld. Un1., Weld. Res. Supp. 22, Figures $34,35,40,42$, (1943).

60. Kouwenhoven, W.B., and Jones, T. Benjamin, "Arc Phenomena With Electrodes Moving at High Speed," Weld. Jn1., Weld. Res. Supp 27, 470s-475s (1948).

61. Jones, T.B., and Kouwenhoven, W.B., "Heat Effects in Anode Spots of High-Current Arcs," Weld Jn1., Weld. Res. Supp 28, 461s-465s, (1949). 
62. Goodfellow, R., "Problems and Developments When Welding In Deep Water," Underwater Welding for Offshore Installations, The Welding Institute, Abington Ha 11, Cambridge, England, Paper 6, pp. 63-72, (1977).

63. Stalker, W.A., and Salter, G.R., "A Preliminary Study of the Effect of Increased Pressure on the Welding Arc," Paper No. RR-SMT-R-7504, The Welding Institute, Abington Ha11, Cambridge, England, 2-5, (1975).

64. Pfluger, Allan R., Boettcher, C.W., and Allen, G., "Gas Tungsten-Arc Welding at Pressures Greater Than Atmospheric," Presented at AWS 1968 Fall Meeting. Not published.

65. Stehle, H., "Welding in Pressurized Inert Gas," Progress in Zircaloy-4 Canning Technology for PWR-Fuel, Fourth United Nations International Conference on Peaceful Uses of Atomic Energy. Geneva, Switzerland, September 6-16, 1971, AED-Conf-71-100-26, Germany, 11-12, May 1971.

66. Goodfellow, R., "Problems and Developments When Welding In Deep Water," Underwater Welding For Offshore Installations, The Welding Institute, Abington Ha11, Cambridge, England, Paper 6, pp. 63-72, 1977.

67. Cobine, J.D., Gaseous Conductors, Dover Publications, 352-363, (1965).

68. Slepian, J., "The Transition From Glow to Arc Discharge at Atmospheric Pressure," Jnl. Frank. Inst., 201, 77-90, (1926).

69. Cobine, J.D., Gaseous Conductors, Dover Publications 352, (1958).

70. Op. cit., 349.

71. Herm, Th Simon, "On the Dynamics of the Electric-Arc Events and on Electric-Arc Hysteresis," Phys. Zeit. 6, 297-319, (1905).

72. vonEnge 1, A., Ionized Gases, Oxford Univ. Press, 274, (1965).

73. Ludwig, H.C., "Plasma Energy in Gas-Shielded Welding Arcs," Weld. Jnl. Weld. Res. Supp., 38, 296s-300s, (1959). (Virtually all of article included.)

74. Langmuir, I., "Atomic Hydrogen Arc Welding," General Electric Review 29, 160-168, (1926).

75. Bjerrum, N., "Dissociation and Specific Heat of Carbon Dioxide at Very High Temperatures as Determined by the Explosion Method," Berlin. Z. Physik. Chem., 79, 537-550, (1912).

76. Saha, M.N., and Srivaslava, B.N., "A Treatise on Heat," Indian Press, Calcutta, India, (1935).

77. Busz-Peuckert, G., and Finkelnburg, W., "Zum Anodenmechanismus des Thermischen Argonbogens," Z. Physik., 144, 244, (1956).

78. Maecker, H., "Ergeb. der Exact. Naturwiss," Band 25, Spring-er-Verlag, Berlin, (1951).

79. Drawin, H.W., "Wirkungsquerschnitte von Neutralen Wasserstoff-, Hel ium- und Argonatomen Gegenuber Electronenstrob," Z. Physik., 146 , 295, (1956). 
80. Gvosdover, S.D., "Mobility and Mean Free Path of Electrons in the Positive Column," Phys. Z. Sowjet 12, 164, (1937).

81. Chapman, S., and Cowling, T.G., The Mathematical Theory of Non-Uniform Gases, Cambridge University Press, (1953).

82. Spitzer, L., and Harm, E., "Transport Phenomena in a Completely Ionized Gas," Phys. Rev., 89, 977, (1953).

83. vonEnge1, A., Ionized Gases, Oxford Univ. Press, Chap 9, (1955).

84. Hocker, K.H., and Finkelnburg, W., "Theorie der Hochstrombogensaule," Z. Naturforsch, 1, 305, (1946).

85. Wienecke, R., "Uber das Geschwindifkeitsfeld der Hochstromkohlebogensaule," Z. Physik. 143, 128, (1955).

86. Hocker, K.H., and Schulz, P., Ibid., 260-270, (1949).

87. King, L.A., Report of International Symposium on Electric Discharge in Gases, The Hague, Martinus Nijhoff, (1955).

88. Haynes, J.R., "The Production of High Velocity Mercury Vapor Jets by Spark Discharge," Phys. Rev., 73, 891, (1948).

89. Larche, K., Lichttechnik, 2, 41-42, (1950).

90. Ludwig, H.C., "Metal Transfer Characteristics in Gas-Shielded Arc Welding," Weld. Jn1., Weld Res. Supp., 36, 23s-26s, (1957).

91. Muller, A., Greene, W.J., and Rothschild, G.R., "Characteristics of Inert-Gas-Shielded Metal-Arcs," Weld. Un1., 30, 717-723, (1951).

92. Maecker, H., Report of International Symposium on Electric Discharges in Gases, The Hague, Martinus Mijhoff, (1955).

93. Post., R.F., "Controlled Fusion Research - An Application of the Physics of High Temperature Plasmas," Re. Mod. Phys., 38, 338, (1956).

94. Morton, H.S., and Gage, R.M., "Arc Properties in the Five Rare Gases," Symposium on Arcs in Inert Atmospheres and Vacuum, edited by Kuhn, W.E., John Wiley, New York, (1956).

95. Eckert, E.R., and Pfender, E., "Plasma Energy Transfer to a Surface With and Without Electric Current," Weld. Jnl., Weld Res. Supp., 46, 471s-480s, (1967). (Virtually all of article included.)

96. Lees, L., "Laminar Heat Transfer Over Blunt-Nosed Bodies at Hypersonic Flight Speeds," Jet Propulsion, 26, 259, (1956).

97. Fay, J.A., and Ridde11, F.R., "Theory of Stagnation Point Heat Transfer in Dissociated Air," Jnl. Aeronaut. Sci., 25, 73, (1958).

98. Eckert, E.R.G., and Tewfik, O.E., "Use of Reference Enthalpy in Specifying the Laminar Heat Transfer Distribution Around Blunt Bodies in Dissociated Air," Jn1. Aerospace Sci., 27, 464, (1960).

99. Penski, K., "Variables of State and Transport Properties of an Argone Plasma," Chem. Ing. Technik., 34, 84, (1962). 
100. Park, C., "Heat Transfer from Nonequilibrium Ionized Argon Gas," AIAA Un1., 2, 169, (1964).

101. Pytte, A., and Williams, A. R., On Electrical Conduction in a Non-Uniform Helium Plasma, ARL 63-166.

102. Shoeck, P.A., Eckert, E.R.G., Wutzke, S.A., An Investigation of the Anode Losses in Argon Arcs and Their Reduction by Transpiration Cooling, (Interim Tech. Report), ARL 62-341.

103. Pfender, E., Eckert, E.R.G., and Raithby, G., "Energy Transfer Studies in a Wall Stabilized Cascaded Arc," Proceedings of the VII International Conference on Ionization Phenomena in Gases, Vo1. I, 691, (1966), Gradivinska Knjige Publishing House, Beograd, (1966).

104. Finkelnburg, W., and Maecker, H., Electrische Bögen und Thermisches Plasma, Handbuch der Physic, Bd. XXII, Springer-Verlag, (1956).

105. Goldman, K., Proceedings Vth International Conference on Ionization Phenomena in Gases, Munich, 1961, North Holland Publishing Company, Amsterdam, (1962).

106. Maecker, H., "Plasmastromungen in Lichtbogen infolge eigenmagnetische Kompression," Z. Physic, 141, 198,(1955).

107. Wienecke, R., "Uber das Geschwindigkeitsfeld der Hochstromkohlebogensaule," Z. Physik., 143, 128, (1955).

108. Larche, K., Lichttechnik, 2, 41-42, (1950).

109. Schoeck, P.A., An Investigation of the Energy Transfer to the Anode of High Intensity Arcs in Argon, Ph.D. Thes is, University of Minnesota, (1961).

110. Schoeck, P.A., and Eckert, E.R.G., Proceedings Vth International Conference on Ionization Phenomena in Gases, Munich, 1961, North Holland Publishing Company, Amsterdam, (1962).

111. Schoeck, P.A., Modern Developments in Heat Transfer, Academic Press, New York, 353, (1963).

112. Nestor, O.H., "Heat Intensity and Current Density Distributions at the Anode of High Current Inert Gas Arcs," Jnl. Appl. Phys., 33, 1638 , (1962).

113. Wutzke, S.A., Cremers, C.J., and Eckert, E.R.G., The Thermal Analys is of Anode and Cathode Regimes in an Electric Arc Column, August 1963, HTLTR No. 56, Mechanical Engineering Department, University of Minnesota.

114. Eberhart, R.C., The Energy Balance for the High Current Arc, NSFGP $\alpha 2520$, University of California, Berkley, California, (1965).

115. Ecker, G., "Electrode Components of Arc Discharges," Erg.d. exakten Naturwiss, 33, Springer-Verlag, (1961).

116. Rosentha1, D., "The Theory of Moving Sources of Heat and Its Application to Metal Treatments," Trans. Amer. Soc. Mech. Engr., 68, 849, (1946). 
117. Maecker, H., "Messung und Auswertung von Bogencharkteristiken $\left(\mathrm{Ar}, \mathrm{N}_{2}\right), " \mathrm{Z}$. Physik., 158, 392, (1960).

118. Pfender, E., Wutzke, S.A., and Eckert, W.R.G., An Arc Tunnel Facility for the Thermal Analys is of Anode and Cathode Regimes," NAAS CR54080 $(1964)$.

119. Pfender, E., and Eckert, E.R.G., Proceedings of the 1965 Heat Transfer and Fluid Mechanics Institute, 50, (1965).

120. Pfender, E., and Cremers, C.J., "Steadiness in a Plasmajet," AIAA Jn1., $3,1345,(1965)$.

121. Cremers, C.J., and Pfender, E., Thermal Characteristics of a High and Low Mass Flux Argon Plasma Jet," ARL 64-191.

122. Apps, R.L., and Milner, D.R., "Heat Flow in Argon-Arc Welding," Bri. Weld. Jnl. 7, (1955). (About 1/2 pp. 477-478 used.)

123. Hanson, G.R., The Effect of Current Ripple on Direct-Current Welded Aluminum Clad Fuel Elements, HW-70692, pp. 4, 5, 7, General Electric Company, Richland, Washington, June 17, 1961.

124. Correy, Thomas B., Fusion Welding of AISi Bonded Fuel Elements, HW-48978, General Electric Company, Richland, Washington, March 11, 1957.

125. Correy, Thomas B., "Waveshape Effect on Alloying and Arc Stability of A-C Tungsten Inert-Arc Welding," IEEE Appli. and Ind., 56, 171-183, (1961).

126. Schaefer, Johannes, Rectifier Circuits: Theory and Design. John Wiley and Sons, New York, 27, (1965).

127. "Five Axis N/C TIG Welding Machine," Weld. and Met. Fab., 36, 457-459, (1968).

128. Hanford Engineering Development Laboratory, Richland, Washington. 99352. A. Computer Programmed TIG Welder is Used to Produce Circumferential Welds on Hexagonal Fuel Ducts Requiring Variable Filler Wire Feed Rates, Variable Current, and Variable Welding Torch Angülation. Not published.

129. Omar, A.A., and Lundin, C.D., "Pulsed Plasma - G.T.A. Arc: A. Study of the Process Variables," Weld. Jnl., Weld. Res. Sup., 58, 97s-105s, (1979).

130. Oscillogram, Courtesy of Hobart Bros. Co., Troy, Ohio 45373.

131. Boughton, P. High Precision Pulsed TIG-Welding, Proceeding of the Conference on Advances in Welding Processes, April 1970, Welding Institute, Abington Hal1, Cambridge, England, Paper 11, 95-98 (1971).

132. Watkins, P.V.C., The Transistor Controlled D.C. Welding Power Source, Research Report, Misc. 35/10/75, October 1975, The Welding Institute, Abington Hall, Cambridge, England, 1-28 (1975). 
133. Mathews, Litell Bryan, The Effect of Slow and Rapid Current Pulsation on the Metallurgical Structure and-Geometrical Properties of Gas Tungsten-Arc Welds. A thesis in partial fulfillment of the requirements for the degree of Bachelor of Science, p 40, University of Tennessee, (1972).

134. Becker, D.W, and Adams, Jr, C.M., "The Role of Pulse G.T.A. Welding Variables in Solidification and Grain Refinement, "Weld. Inl., Weld. Res. Sup., 58, 1435s-5s-152s, (1979).

135. Information furnished by John Kuhr, Miller Electric Mfg. Co., Appleton, Wisc., 54911.

136. Wyer, R.F., U.S. Patent 2,474,023, Method of Gas Shielded AlternatingCurrent Arc Welding, June 21, 1949. (An excellent treatise on the beneficial effects of high open-circuit voltage.).

137. Selin, Karl I., and Kusko, A., "Experimental Characteristics of 3-Phase Poly Saturable Reactors," AIEE Trans., Vol. 75, Pt. 3, No. 26, 868-871, (1956).

138. Selin, Karl I., "Poly Unit Saturable Reactor," Ibid., 863-867, (1956).

139. Selin, Karl I., U.S. Patent 2,916,689, Saturable Core Regulators, December 8, 1959 .

140. Weed, J.M., U.S. Patent 1,612,084, Alternating-Current Arc Welding, December 28, 1926.

141. Milnes,A.G., Transducers and Magnetic Ampl ifiers, McMillan, London, (1957).

142. Storm, H.F., Magnetic Amplifiers, John Wiley, New York, pp. 113 and 167, (1955).

143. Storm, H.F., "Series Connected Saturable Reactors With Control Source of Relatively High Impedance," AIEE Trans., 66, 1299-1309, (1950).

144. Correy, Thomas B., U.S. Patent 3,068,352, Method for Obtaining an Improved Weld in Inert Arc Welding, December 11, 1962.

145. Vilkas, E.W., "Square Wave Welding," IEEE Trans. Ind. Gen. Appli. Vol. IGA-3, No. 6, 525,530, (1967).

146. Sciacky Bros., Inc., Chicago, Illinois 60638, Pulsed Square Current Oscillograms.

147. Kanevisto, M. and Pullinen, J., "Development in the Design of Welding Plant," Australian Weld. Jn1., 22, 17-19, (1978).

148. Auh1, J., Richard, Program Manager, Evaluate Welding Machine Specifications and Conduct Trials of Two Prototype Units, Technical Study, Final Report, Aluminum Co. of America, Alcoa Laboratories, Alcoa, Pa., 15069, for Union Carbide Corp., Nuclear Div., Oak Ridge, Tennessee, 37830 , prepared under Contract No. 72K-02269V, 9/28/76 issued December 3, 1976. 
149. Oscillograms of Wide Range Balanced Sine Wave Current With Saturable Reactor, Miller Electric Mfg., Co., Appleton, Wisconsin, 54911.

150. Cobine, J.D., Gaseous Conductors, Dover Publications, New York, N.Y., (1958).

151. Oscillograms in Figures 88-101 were furnished by the courtesy of Dr. R. R. Wright, Automated Products, Div., BOC Limited, Milton Keynes, England.

152. Grist, F. J., and Armstrong, F. W., "A New Constant Potential Power Source for Heavy Plate, Deep Groove Welding," Weld. In1., 59, 30-35, (1980).

153. Bunker, T. A., "Multi-Electrodes in SAW With Square Wave AC Power," Weld. Int., 61, 36-40, (1982). 



\section{SUPPLEMENTAL REFERENCES}

1. Chapin, N.A., Cobine, J.D., and Gallagher, C.J., "Tungsten-Thoria Electrodes for Inert Arc Welding," Weld. Jn1., 30, 529-531, (1951).

2. Edels, H., "Properties and Theory of the Electric Arc," The Inst. of Elect. Engr., London, Paper No. 3498, 55-69, (1961).

3. Francis, Gordon, "Ionization Phenomena in Gases," Butterworths Scientific Publications, London, pp. 32-33, 50-51, 59-60, and 158-159, (1960).

4. Pfeil, P.C.L., and Griffiths, L.B., "The Effect of Inclusions on the Arcing Behavior of Metals," Jnl. Nuc. Matls., North Holland Publishing Co., 3, 244-248, (1959).

5. Kenyon, D.M., and Boyce, A.J., "Effect of Cathode Length and Diameter on Tungsten Arc Characteristics," Brit. Weld. Inl., 13, 103-108, (1966).

6. Vegner, O.G., "Increase of Stability of an A.C. Welding Arc," Elect. Tech., U.S.S.R., 252-258, Apri1 1959.

7. Rollason, E.C., "The Electric Arc in Welding: Introductory Survey," Brit. Weld. Jn1., 7, 71-72, (1960).

8. Milner, D.R., Salter, G.R., and Wilkinson, J.B., "Arc Characteristics and Their Significance in Welding," Op cit., 73-78.

9. Salter, G.R., and Milner, D.R., "Gas Absorptions From Arc Atmospheres," Op. cit., 89-100.

10. Wilkinson, J.B., and Milner, D.R., "Heat Transfer From Arcs," Op. cit. 115-128.

11. Pattee, H.E., Survey of Alternating-Current Power Supplies for Welding, AD-465 248 Battelle Memorial Institute, Columbus, Ohio, for Redstone Scientific Information Center, Research Branch, U.S. Army Missile Command, Redstone Arsenal, Alabama, pp. 1-25, November 25, 1965.

12. Pattee, H.E., Effect of Current Wave Form on the Properties of Aluminum Welds, RSIC-402, Battelle Memorial Institute for Redstone Scientific Information Center, U.S. Army Missile Command, Redstone Arsena 1, Alabama, pp. 1-33, April 1965.

13. Gibson, G.J., and Rothschild, G.R., "The Effect of D-C Component in A-C Inert-Gas Arc Welding of Aluminum," Weld. Jnl., Weld. Res. Supp. 27, 496s - 501-s, (1948).

14. Tuthill, R.W., "The Effect of Wave Shape in Inert-Arc Welding Circuits," Weld. Jn1. 27, 785-788, (1948).

15. Sugihara, Eijiro, Researches on the Arc Welding Machine, No. 609, Electrotechnical Laboratory, 2-Chome, Nagata-Cho, Chiyoda-Ku, Tokyo, Japan, pp. 1-70, September 1961. 
16. Winsor, L.P., and Turk, R.R., "A Comparative Study of Thoriated, Zirconated, and Pure Tungsten Electrodes," Weld. Jnl., Weld. Res. Supp. 36, 113-s - 119-s, (1957).

17. Sugihara, E., Kikuchi, S., and Yada, K., "ETL Type Welder," Jap. Weld. Soc. Jnl., Tokyo, 28, 31-36, (1959).

18. Bibliography on High Pressure Arcs, AIEE Bulletin S-62, (1954).

19. Houldcroft, P.T., "Developing Precision Assembly by Welding," 1977 Comfort A. Adams Lecture, Weld. In 1., 56, pp. 15-25, (1977).

20. Bromage, K., "Arc Efficiency and Heat Flow in Inert Gas Welds," Brit. Weld. Jn1., 15, 493-500, (1968).

21. Needham, J.C., and Orton, L.H., "A.C. Argonarc Welding at Less than 50 V.R.M.S. Open-Circuit," The British and Allied Industries Research Association, U.D.C. 621.791.75, May 20, 1952, pp. 1-17. For contact see British Welding Research Association, Cambridge, Abington Hall, England.

22. Jennings, Chas. H., and White, Alfred B., "Magnetic Arc Blow," Weld. Jn1., Weld Res. Supp. 20, 427s - 436s, (1941).

23. Jayarajan, T.N., and Jackson, C.E., "Magnetic Control of Gas Tungsten-Arc Process," Weld. Inl., Weld. Res. Supp. 51, 377s - 385s, (1972).

24. Hicken, G.K., Stucky, N.D., and Randa11, H.W., Applications of Magnetically Controlled Welding Arcs," Weld. Jn1., 55, 264-267, (1976).

25. Hicken, G.K., and Jackson, C.E., "The Effects of Applied Magnetic Fields on Welding Arcs," Weld. Jnl., Weld. Res. Supp. 45, 515s - 524s, (1966).

26. Dickerson, Paul B., Program Manager, Evaluate and Develop Automatic A.C. (HF) - TIG Welding of Aluminum Pipe, Technical Study - Phase I, Prepared under Contract No. 72K-93118V, for Union Carbide Corp Nuclear Division, Oak Ridge, Tennessee, UCC Specification JS a YEM a 42446 - 31, Rev. B, Government Contract No. W-74-05, Final Report. September 20, 1976, Aluminum Company of America, Alcoa Laboratories, Alcoa Center, Pennsylvania 15069.

27. Sager, R.K., Michnuk, P.R., and Mahan, G.W., Evaluate and Develop Automatic TIG Welding of Aluminum Pipe, Technical Study - Phase II, Prepared under Contract No. 72K-02200V for Union Carbide Corp Nuclear Division, Oak Ridge, Tennessee, UCC Specification JS - YEM 42446-31, Rev. A. Government Contract No. W-7405. Final Report. September 30, 1976, Aluminum Company of America, Alcoa Laboratories, Alcoa, Pennsylvania 15069.

28. Dickens, R.G., and Pinfold, B.E., "Investigations in Pulsed Tungsten Inert-Gas Welding," Paper 11, Proceedings of the Conference on the Advances in Welding, April 1970, The Welding Institute, Abington Hall, Cambridge, England, 63-67 (1971). 
29. Vagi, J. J., Mishler, H. W., and Randa11, M. D., "Pulsed-Current ArC Welding Processes," Defense Metals Information Center, Battelle Memorial Institute, Columbus, Ohio, 43201. DMIC Memorandum 250. 1-24, September 1979 .

30. Lucas, W., "Exploitation of Transistor Power Sources in Arc Welding," Research Bullet in R140/11/77, November 1977, The Welding Institute, Abington Hall, Cambridge, England, 304-310, (1977).

31. Boughton, P., "High Precision TIG Welding," Proceedings of the Conference on Advances in Welding Processes, April 1970, Welding Institute, Cambridge, England, Paper 11, 95-98 (1971).

32. Dugdale, R. A., "Engineering With Glow Discharge," Science Journal, 4, 40-45 (1968).

33. Soehngen, Erich, E., and Bauder, Uwe, "Investigation of Plasmas at Very High Pressures," Aerospace Resaerch Laboratories, Air Force Systems Command, Wright Patterson Air Force Base, Ohio, Inst. of Environmental Sciences, Proceedings, 276-280, (1969). 


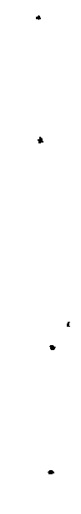


APPENDIX A

\section{THE GLOW DISCHARGE AND ELECTRIC ARC COMPONENTS*}

The gaseous discharges of interest in welding are:

\section{Low Pressure Glow Discharge}

Appearance of the low pressure glow discharge starts on decreasing pressure at approximately a few centimeters (one inch) of mercury absolute pressure, depending on the electrode configuration and gaseous element(s) within the enclosing vessel. Its principal characteristics are no cathode spot and no anode melting. An excellent example is a fluorescent tube lamp. Al1 visible light ceases at approximately $1 \times 10^{-3} \mathrm{~mm}(0.00004 \mathrm{in.})$ mercury absolute pressure depending on electrode configuration, gaseous element(s), and the enclosing vessel material. The distribution of parameters between the electrodes is shown in Figure 87.150

\section{Low Pressure Arc}

The gas temperature of the low pressure arc is never more than a few hundred degrees centigrade (approximately $400^{\circ} \mathrm{F}$ ) while the electron temperature may be as high as $40,000 \mathrm{~K}$ (approximately $72,000^{\circ} \mathrm{F}$ ). It has a true cathode spot which furnishes the electron current and a true anode spot which can produce melting if anode mass, melting point, and thermal conductivity are in the proper proportions. In Figure 1 is shown the voltage drop along a true arc. The low pressure arc exists between approximately a few centimeters (approximately 1 in.) of mercury absolute pressure to approximately twenty centimeters (approximately 8 in.) of mercury absolute pressure depending on electrode configuration, electrode mass, and gaseous element(s) within the enclosing vessel.

* For a more detailed discussion of arc components, refer to "Gaseous Conductors," J. D. Cobine, Dover Publications, Chapters 8 and 9 (1958) New York. 


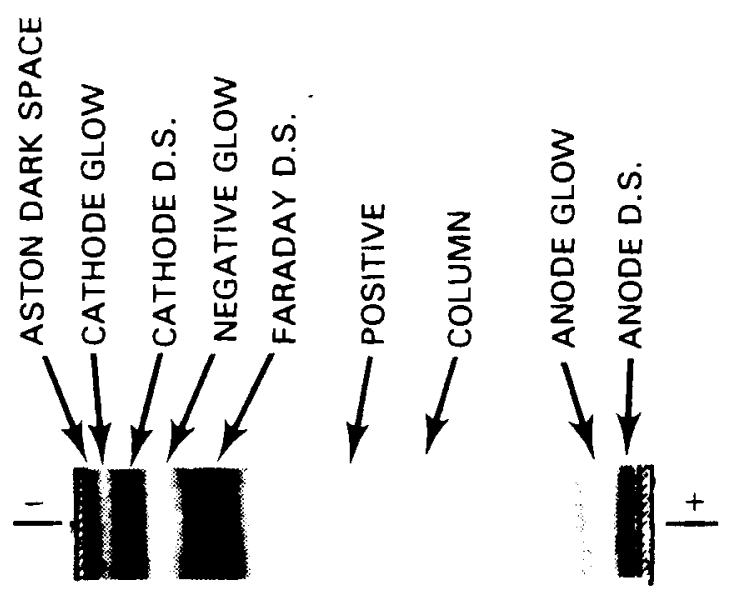

LIGHT

INTENSITY

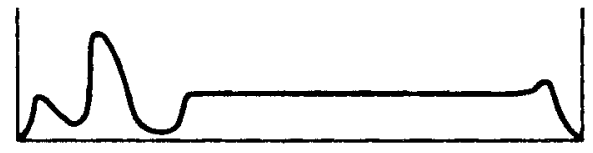

VOLTAGE

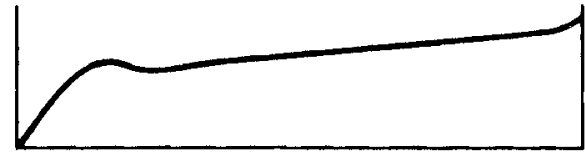

VOLTAGE

GRADIENT

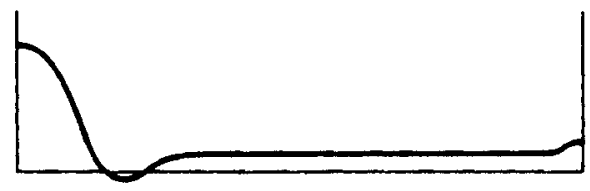

NET CHARGE

DENSITY

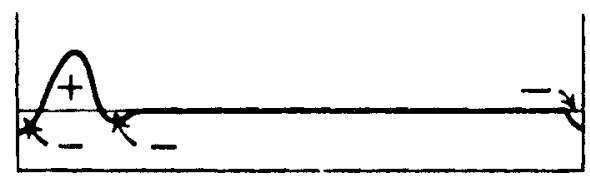

POSITIVE

CHARGE

DENSITY

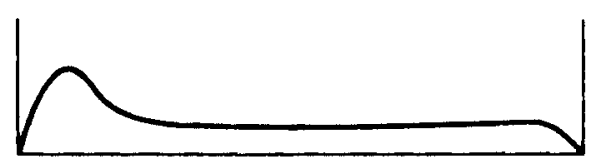

NEGATIVE

CHARGE

DENSITY

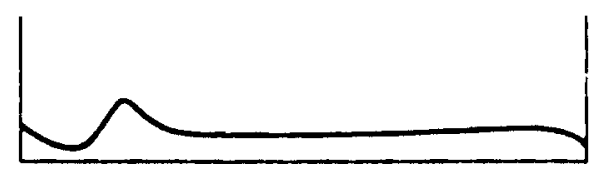

ELECTRON CURRENT DENSITY

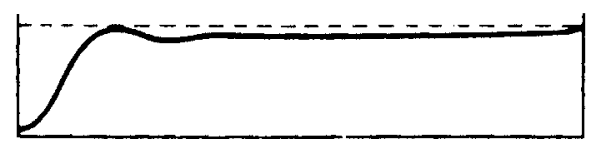

FIGURE 87. Characteristics of the Low Pressure Glow Discnarge 150 


\section{High Pressure Arc}

The gas temperature of the high pressure arc column is usually in the order of 5000 to $6000^{\circ} \mathrm{K}\left(9000\right.$ to $\left.10,800^{\circ} \mathrm{F}\right)$. Formation of the high pressure arc takes place on rising pressure at approximately twenty centimeters ( 8 in.) of mercury absolute pressure, at which pressure the electrons, ions, and gas atoms are in thermal equilibrium (equal temperature). Pressures to 1000 atmospheres $\left(10,000 \mathrm{~N} / \mathrm{cm}^{2}\right)$ have been investigated and no deviation from a true high pressure arc has been found. In the high pressure arc (atmospheric pressure) the cathode spot occupies a stable position on the electrode and when melting takes place by electron bombardment the anode spot forms and anchors in a stable position.

The low pressure glow discharge, the low pressure arc, and the high pressure arc have cathode and anode fall spaces extending axially out toward the opposite electrode. Connecting the fall spaces is the column. The column is simply a conducting path between the anode and cathode fall spaces of the low pressure glow discharge, the low pressure arc, and the high pressure arc. Its length can vary from virtually zero to virtually infinity.

The cathode fall space is a region of very high positive ion density and produces the greatest voltage drop in the high pressure arc (atmospheric pressure) providing the heat to the cathode by positive ion bombardment to produce the required electron emission. As the gas pressure is lowered, the high pressure arc changes to a low pressure arc, followed by a low pressure glow discharge. The arc cathode and anode regions elongate to the point of visibility along the arc axis as the low pressure glow discharge forms. At roughly one millimeter (0.0394 in.) mercury absolute pressure, depending on the gas, the fall spaces consist of alternate light and dark bands. The bands, of varying intensity and color, start at the cathode surface and extend along the arc axis. The known bands, Figure 87,150 starting at the cathode surface are:

- Aston dark space

- Cathode glow

- Cathode dark space 
- Negative glow

- Faraday dark space

The dark spaces are not totally dark, only dark relative to the bright glowing regions where ionization and excitation processes are much more active.

The anode end of the anode fall region of the high pressure arc has a high density space charge of electrons. They come from the cathode, ionization of the gas, and the anode. In addition, positive ions, produced by electron collision, are produced in the anode fall region. It is at the anode that the essential positive ion current that heats the cathode by bombardment to produce thermionic emission of electrons is established. At the anode surface is a dark region called the anode dark space, followed by a bright glow, called the anode glow. Their production depends on the gas and especially the value of the discharge current.

From the mass of the material in the literature on the low pressure glow discharge and the high pressure arc, it appears that the general belief is that as the pressure of the low pressure glow discharge rises, all of the spaces at the anode and cathode are compressed into such a thin, possibly homogenous, space at each electrode as to become invisible. During the rise of pressure there is a transition from the low pressure glow discharge to the low pressure arc discharge and then to the high pressure arc discharge. Each type of discharge has its own characteristic anode and cathode fall regions. 


\section{APPENDIX B}

COMPOSITION OF INCONEL ALLOY 600

$\mathrm{Ni} 76 \%$, Fe $7.2 \%$, Cr $15.8 \%$, C $0.04 \%$, Mn 0.20\%, Si $0.20 \%$ 



\section{APPENDIX C}

\section{COMPOSITIONS OF ZIRCALOYS-2 AND -4}

\section{$\mathrm{Zr}-2$}

A1 75ppm B 0.5ppm Cb $0.5 p p m$ C 270ppm Cr 0.06-0.014\%

Co 20ppm Cu 50ppm Hf 200ppm H 40ppm Fe 0.07-0.17\%

$\mathrm{Pb} 0.130 \mathrm{ppm} \mathrm{Mg} 20 \mathrm{ppm} \mathrm{Mn} 50 \mathrm{ppm}$ Mo 50ppm Ni 0.03-0.07\%

N 80ppm Si 100ppm Sn 1.25-1.65\% Ti 50ppm W 100ppm

V 50ppm

$\mathrm{Zr}-4$

Cr 0.05-0.15\% Fe 0.18-0.24\% Ni 0.007 Max. Sn 1.20-1.70\%

other elements are the same as for $\mathrm{Zr}-2$ 


\section{APPENDIX D \\ ALTERNATING-CURRENT BALANCING}

Due to the high thermal conductivity of the aluminum and the thermal electron emission difference between the tungsten electrode and the aluminum workpiece, the amount of current flowing when the tungsten electrode is negative is greater than when the aluminum workpiece is negative. In welding, this is known as unbalanced current. It may be corrected by connecting a capacitor of several hundred microfarads in series with the welding current circuit. The nature of the capacitor is such that it will pass equal amounts of current in each direction and still conduct current continuously. Solid state diodes back to back with a parallel adjustable resistor will perform the same function but the resistor has to be adjusted for each current change. A six-volt storage battery connected in series with the welding current circuit and having the negative terminal connected to the workpiece will provide a partial correction of the unbalance. The storage battery will require recharging occasionally.

Higher open-circuit voltages tend to balance the current but will not do so completely. 


\section{APPENDIX E}

\section{NATURAL IONIZATION IN AIR}

Electrons released by cosmic particle bombardment of atoms in the air, and by natural radioactive elements in the air, electrodes and surrounding hardware provide the initial electrons required for arc starting. This in turn reduces the breakdown voltage of the air and shielding gas. Since the release of electrons by cosmic particle bombardment of gas atoms is a random process, the minimum breakdown voltage between the electrodes and the workpiece may not be the shortest path between the point of the electrode and the workpiece. Thus, the arc may initiate at some place along the electrode other than the point. 


\section{ADDENDUM I}

\section{HIGH PERFORMANCE, PULSED-CURRENT WELDING POWER SUPPLIES}

\section{INTRODUCTION}

The advent of the two ampere transistors, when connected into large parallel groups, permitted the handling, metering, and very high rates of switching of currents in the welding range and thus the development of the first high performance welding power supplies. This has been further enhanced by the development of the 200 and 400 ampere transistors. It has not been found in the literature by whom and where the first transistorized high performance welding power supply was produced. It seemed suddenly that there were a number of different makes on the market, mostly of United States manufacture. The actual number on the world market is not known but it is in excess of the original four. The one developed in England $(132)$ is now licensed for manufacture in England, the United States, and Japan.

Two circuit features that are common to the first three high performance welding power supplies are:

- An insulating transformer that insulates the rectifier and welding power supply from the alternating-current supply and also produces the required voltage for welding.

- The rectifier is a three-phase, full wave bridge.

Beyond the rectifier there are at least three types of circuits:

- The first type of circuit employed is a pulsing type of circuit with the peak and background values of current maintained at constant current values. The desired value of welding current is obtained by changing the time period of the current pulses which produce the desired current from the average value of the two currents.

- The second type is very similar to the first type except that the pulsing of the current is at a high frequency and is performed slightly differently. At low values of welding current, the pulses go slightly 
below the zero of the direct-current and thus produce a very small alternating-current in addition to the pulsing. There is a noise produced by the arc due to the current pulsing.

- The third type 132 filters the direct-current output of the rectifier with a very large bank of capacitors which perform two functions:

- Removes the ripple from the rectified alternating-current.

- Assists in providing current rise and decay times of $10^{6}$ amperes per second.

The welding current in this type of high performance welding power supply is controlled directly by the transistors, using them as a throttle in the welding current circuit.

- A fourth type of high performance power supply that appears to be on its way to market converts the alternating-current supply power directly to direct-current wi th a three-phase bridge rectifier. The directcurrent is then converted to a single phase high-frequency current whose frequency is varied to produce the required value of welding current. A two winding high-frequency transformer is used to insulate the welding current from the incoming alternating-current. The output of the high-frequency transformer passes through a single-phase two diode full wave rectifier to supply the direct-current for welding. Since it is a single-phase rectifier, there is a ripple in the directcurrent which makes noise as it passes through the arc. In addition, there is noise produced by the magnetic core. The advantages of this type over the other three are:

- Higher overall electrical efficiency from the three-phase input to the direct-current output.

- The physical weight of the high performance welding power supply is reduced substantially.

- The cost of the high performance welding power supply is reduced substantially. 


\section{CURRENT RIPPLE AND CURRENT PULSING}

In the early development of precision welding it appeared that the most desirable form for direct-current was a ripple-free current with very high rates of current rise and decay to permit rapid arc starting, current programming, and current pulsing.

Though no reasons have appeared in the literature for the designers of high-performance direct-current welding power supplies using high rates of current pulsing in the welding current over the ripple-free current, the various types of high-performance direct-current welding power supplies are operating side by side on the same job-producing equal high-quality welds.

There is no evidence that there is any problem in starting the arc with high-frequency current when using pure helium shielding gas in gas tungsten arc welding with any of the four types of high-performance direct-current welding power supplies.

A similar situation exists in high-performance alternating-current welding. In one case ${ }^{145}$ it was found that what appeared ${ }^{148}$ to be a very undesirable alternating-current pulse formation produced the highest quality weld.

\section{PULSE FORMATION OF DIRECT-CURRENT}

It is desirable in researching the effects of current pulsing and pulse shaping on weld quality to use a ripple-free, high rate of current rise and decay welding power supply for the following reasons:

- A ripple-free source is essential for producing and reproducing reference welds.

- The exact pulse shapes can be produced and reproduced with a computer.

There is considerable interest in the possibility that weld quality produced by a shielded metal arc, gas tungsten arc, gas metal arc, electrogas, and electroslag welding may be improved by pulse timing and shaping of the welding current. 


\section{PULSE SHAPE SYNTHESIZING}

By computer synthesizing it is possible to tailor virtually any geometric shape, the shapes being made up of a great number of short time segments. Applying this principle to ripple-free welding current, pulse shapes can be tailored to any shape that can be made up of pulses that are one millisecond long. Any time the current changes magnitude or direction, there is a one millisecond delay between the original current and the change. Though the following light beam oscillograms all show linear up and down slopes, 151 other slopes such as exponential or step may be produced.

In Figure 88 is shown an oscillogram of the virtually ripple-free current of the third type of high performance direct-current welding power supply. There is no ripple detectable.

Figure 89 shows a square pulse of current with the maximum rate of rise and decay of current, $10^{6}$ amperes per second. The rates of rise and decay are greater than the writing speed of the light beam, thus there is no ordinate trace.

A square wave with a pulsation rate eight times the rate in Figure 89 is shown in Figure 90.

Shown in Figure 91 is a symmetrical sawtooth pulse. There is a one millisecond delay at both the peak and the base before the current reverses.

In Figure 92 is shown a series of sawtooth pulses with a background current hold of five milliseconds.

A simple, slow rise pulse of current is shown in Figure 93. Again the rate of current decay is faster than the writing speed of the light beam.

In Figure 94 is shown a fairly fast simple shape of current pulse having a penetration peak of current.

Shown in Figure 95 is a fairly slow pulse of current having a penetration peak that is held at its maximum current for forty milliseconds. 
Introducing pulsing into a shaped current pulse during downsloping is shown in Figure 96. Pulsing may be applied to any or all portions of a sloped current pulse. The limiting factor is that the base value of the pulsing current wi11 always be the background current of the shaped wave.

Figure 97 is a fast pulse of current with a high-frequency sawtooth pulse of current superimposed on it. The magnitude of the sawteeth are proportional to the values of the current.

The same square pulse shown in Figure 97 is shown in Figure 98 with a sine wave of current superimposed on the base and peak current pulses. As in the previous figure, the magnitude of the sine waves are proportional to the magnitude of the current pulses.

In Figure 99 are shown the same square pulses of current as in the two previous figures with a square wave of current superimposed on it.

The effect of increasing the frequency of the superimposed sine wave by a factor of ten on the previous square waves is shown in Figure 100.

Square current pulses as shown in the previous figure are shown in Figure 101 with a sine wave of current superimposed on it of ten times the previous frequency. The one-tenth millisecond pulses used to tailor the sine wave are visible.

\section{CERTIFICATION OF HIGH PERFORMANCE WELDING POWER SUPPLIES}

The first three types of high performance direct-current welding power supplies have been certified for all phases of welding in the aerospace, nuclear power, and piping and pressure vessel fields regardless of the severity of the requirements. Development of the fourth type has not advanced to the point of certification. 


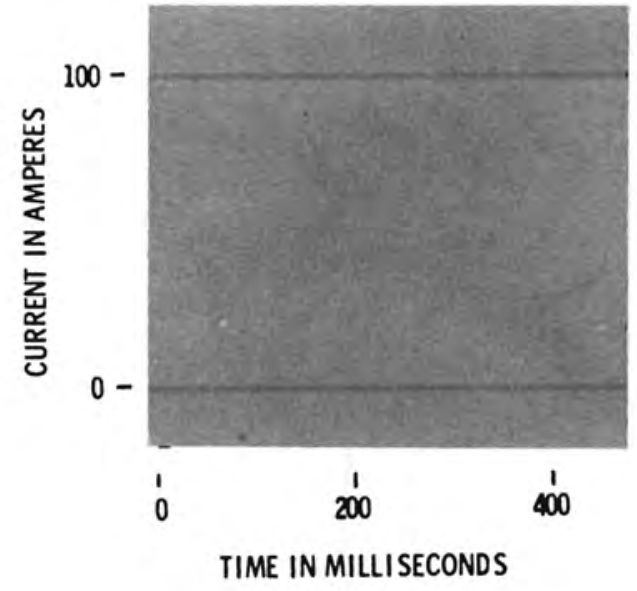

FIGURE 88. Steady State Welding Current

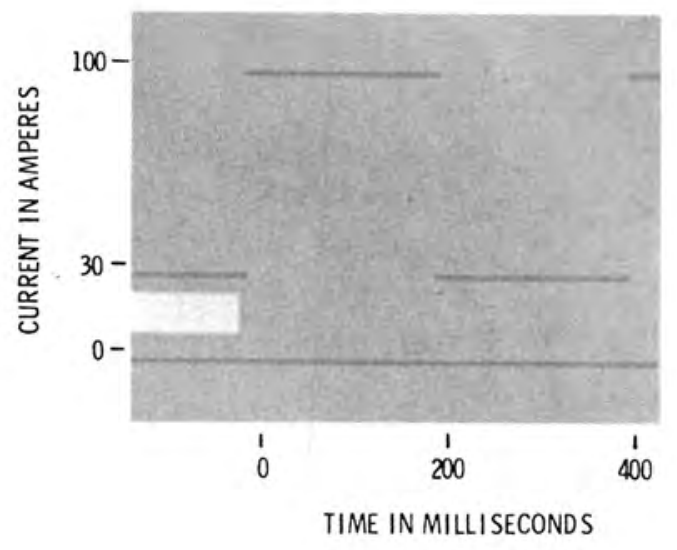

FIGURE 89. Square Wave Slow Pulsing 


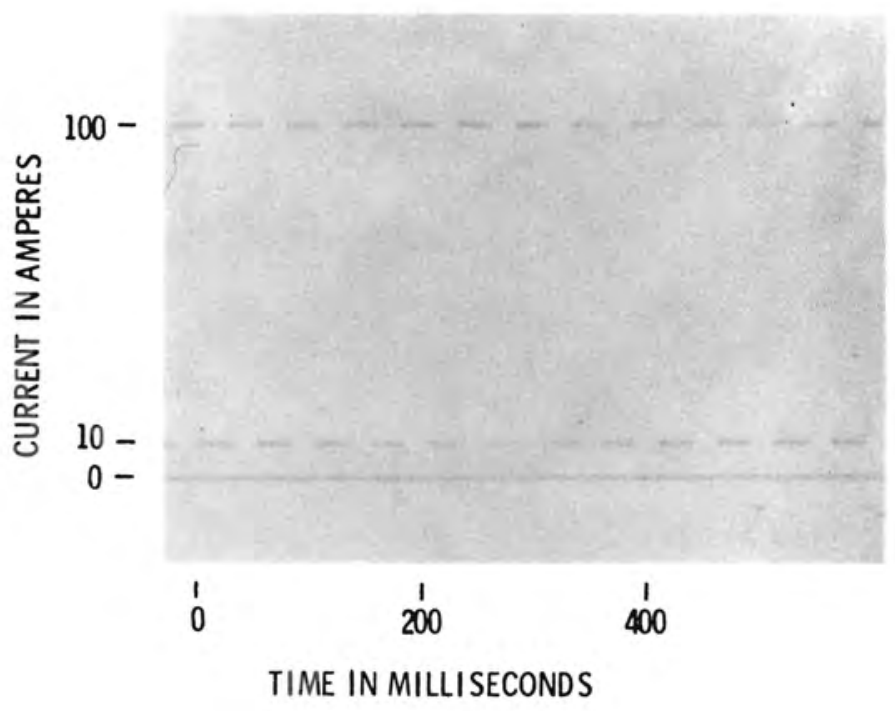

FIGURE 90. Square Wave Fast Pulse

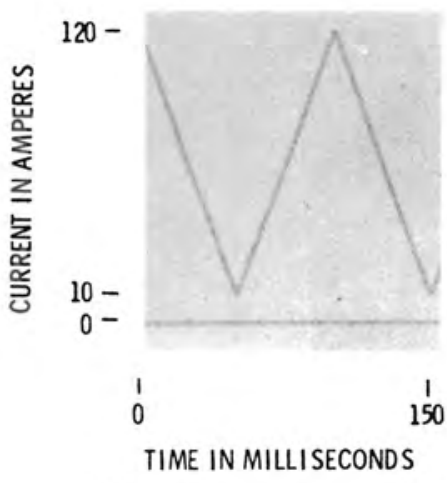

PEAK HOLD 1 MS

BACKGROUND HOLD I MS

FIGURE 91. Slow Sawtooth Pulsing 


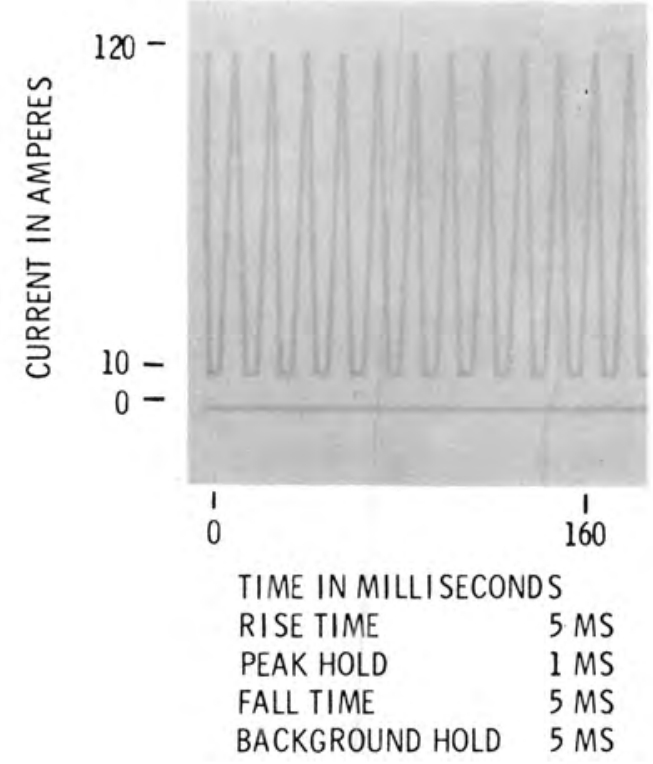

FIGURE 92. Fast Triangular Pulsing

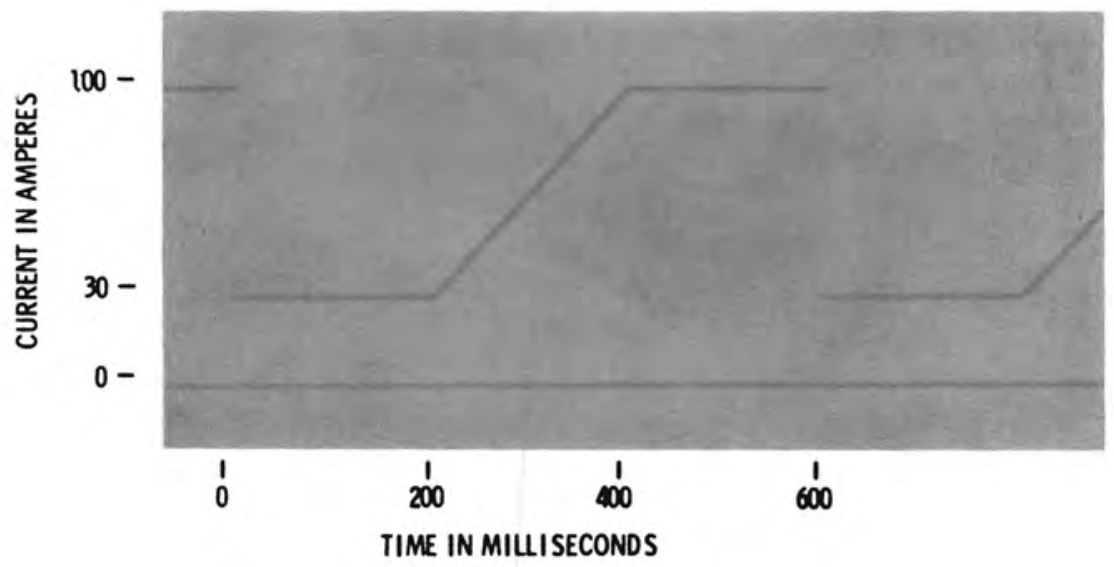

FIGURE 93. Pulse With Upslope 


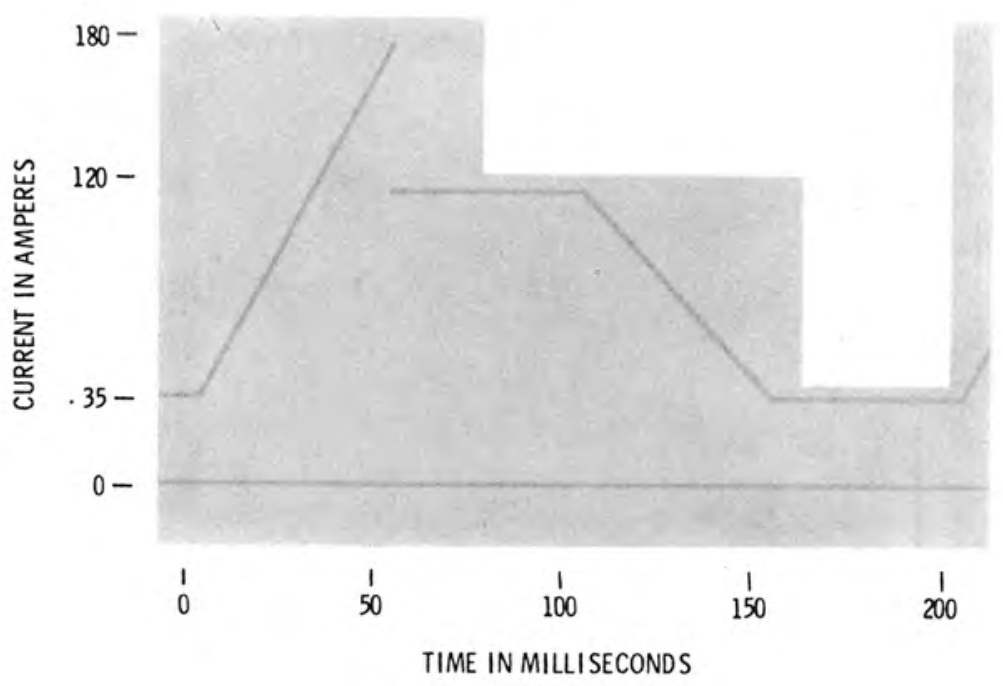

FIGURE 94. Linear Sloped Penetration Peak Pulse

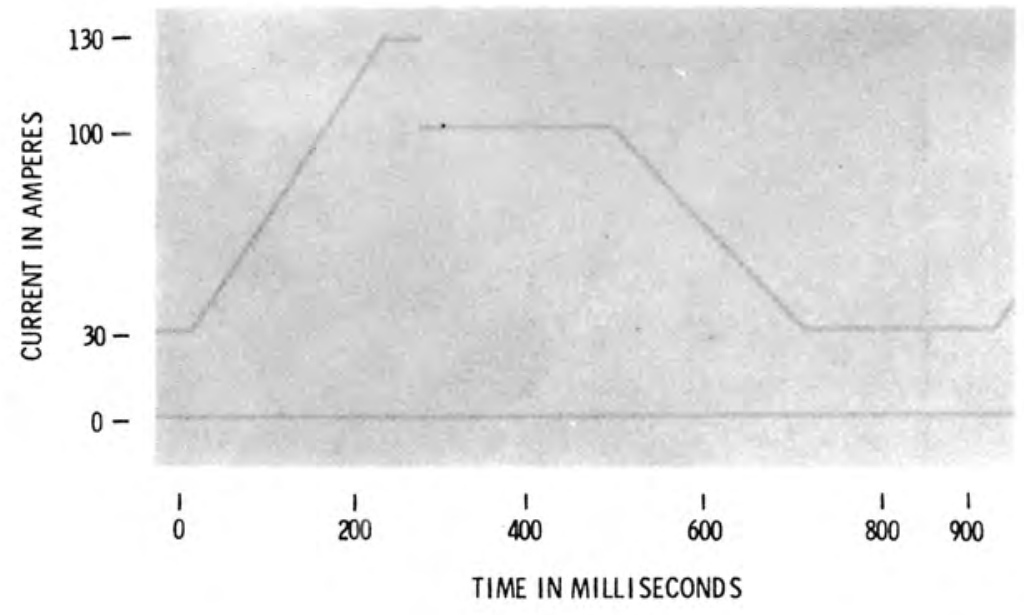

FIGURE 95. Penetration Peak Pulse With Peak Hold 


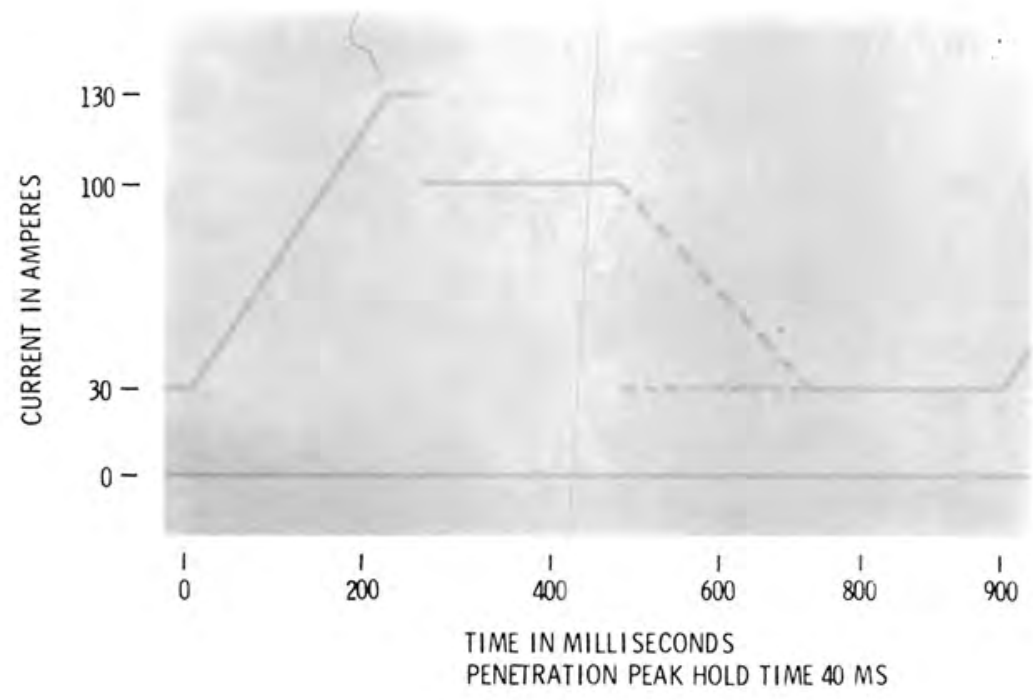

FIGURE 96. Linear Sloped Penetration Peak Pulse With Hold and Down Slope With Pulsing

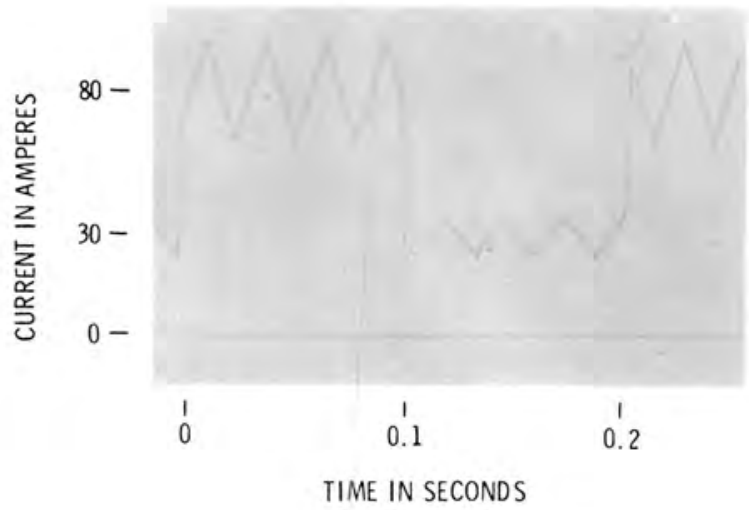

FIGURE 97. Square Current Pulses With Sawtooth Pulses Superimposed on Peak and Background Pulses 


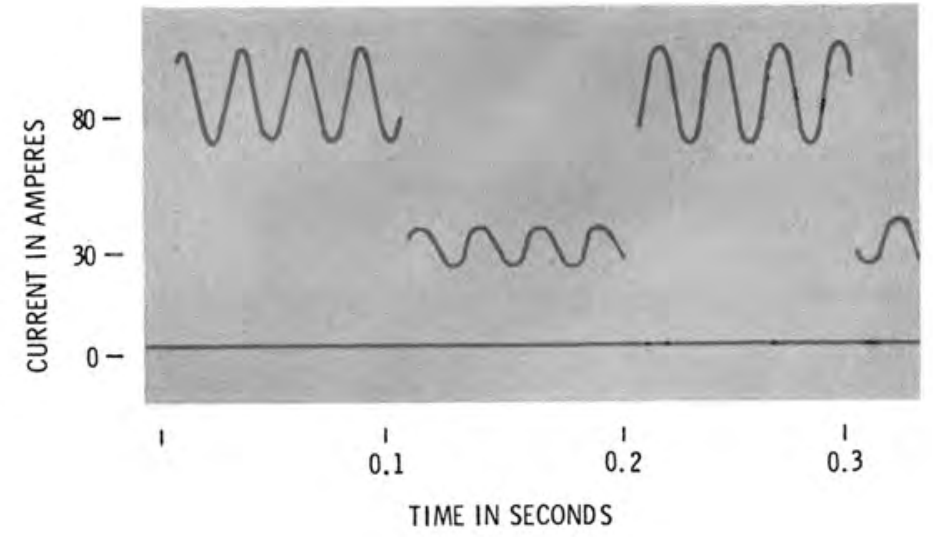

FIGURE 98. Square Current Pulses With Sine Wave Pulses Superimposed on Peak and Background Pulses

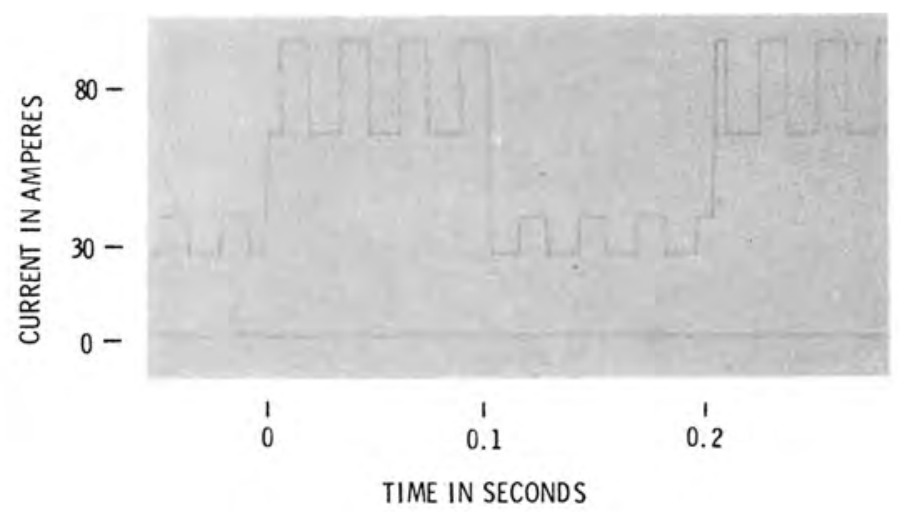

FIGURE 99. Square Current Pulse with Square Pulses Superimposed on Peak and Background Pulses 


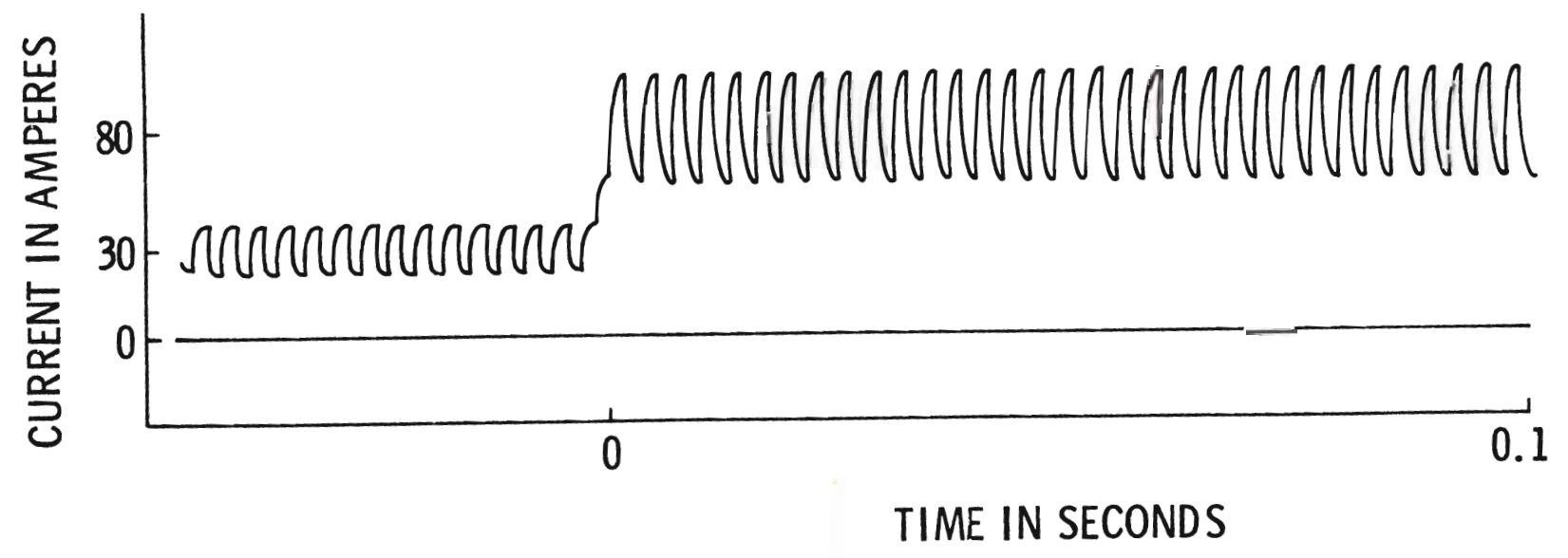

FIGURE 100. Square Current Pulses With Pulses Similar to Sine Waves Superimposed on Peak and Background Pulses

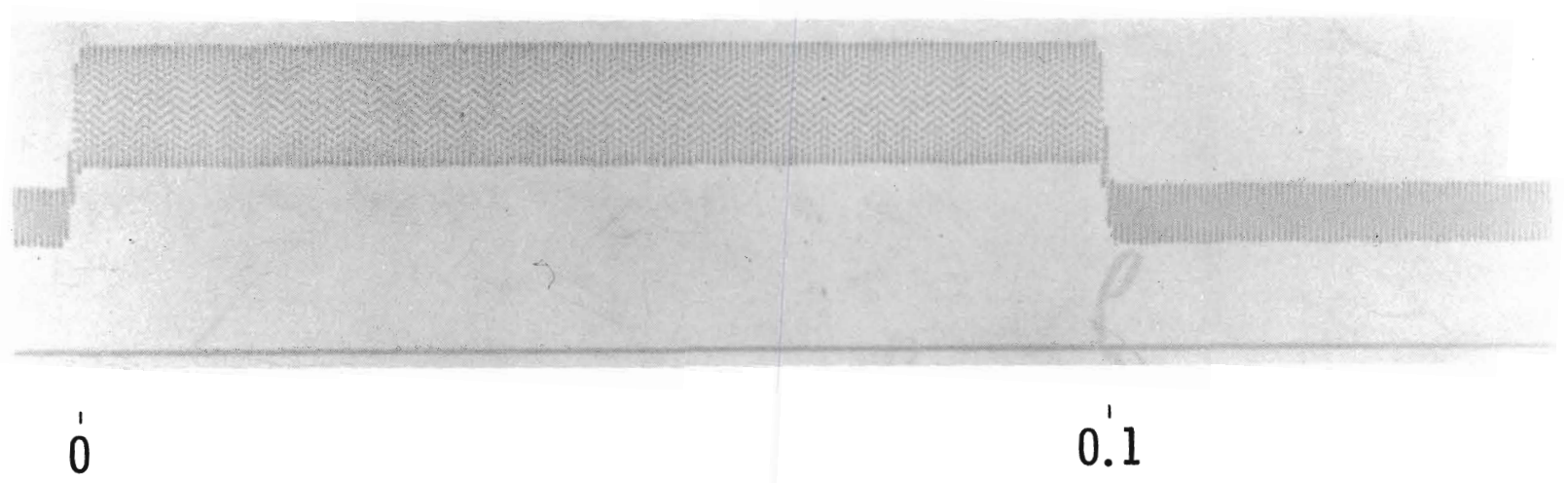

TIME IN SECONDS

FIGURE 101. Square Current Pulses With Sine Wave of Ten Times the Frequency of Figure 100 Superimposed on Peak and Background Pulses Showing the Building Block Pulse of One Millisecond Duration Used to Tailor the Pulses 
ADDENDUM II

\section{ELECTRICAL EFFICIENCIES OF WELDING POWER SUPPLIES}

Due to the energy shortage and the escalating price of electrical energy, interest is being generated in improving the electrical efficiency of all welding power supplies.

Efficiencies of existing drooping current welding power supplies, from the general purpose to the high performance ones, vary from about 20 per cent to 95 per cent in any performance classification.

Generally speaking, the electrical efficiency of drooping current welding power supplies may be determined by the method or circuit used to set the welding current and in addition the open-circuit voltage. The amount of voltage droop between the open-circuit and the arc voltage, for a finite value of welding current, multiplied by the welding current gives a good measure of the electrical losses, providing that some of the drop does not occur across an alternating-current component carrying welding current such as a saturable reactor or magnetic amplifier. In the latter case the quadrature drop due to the inductance must be deducted.

In selecting a welding power supply for its electrical efficiency, the electrical efficiency has to be given consideration after the electrical characteristics, such as rate of change of current, ripple current, opencircuit voltage, etc., since high electrical efficiencies are not currently available in all performances of welding power supplies.

One source of current for direct-current welding that has not been used is an amplidyne. It is a motor-driven direct-current generator in which the generator has been modified. The device may have more than two independent shunt fields. In conjunction with the shunt fields the modified connective circuit produces high rates of rises and decay of the output current. If used as a welding power supply, the difference between the open-circuit voltage and the arc voltage would effectively disappear as the control field would drop the open-circuit voltage, upon arc starting, 
to that required to maintain the arc at its required voltage and current.

Remaining are the copper, friction, windage in the generator, and the motor losses. Usually these are quite small in a unit of this type. 


\section{ADDENDUM III}

\section{SQUARE WAVE ALTERNATING-CURRENT}

NARROW GROOVE SUBMERGED ARC WELDING

A recent development in the automatic narrow groove submerged arc welding is the use of constant potential square wave alternating-current for melting the electrode. 152 Shown in Figure 102A is a schematic oscillogram of a sine wave and Figure $102 \mathrm{~B}$ an actual oscillogram of square wave of submerged arc welding currents. It will be noted that the areas under the positive and negative pulses are virtually equal. This is due to the fact that both the electrode wire and the weld puddle have virtually the same thermal electron emission characteristics, thus producing virtually no rectification or current unbalance.

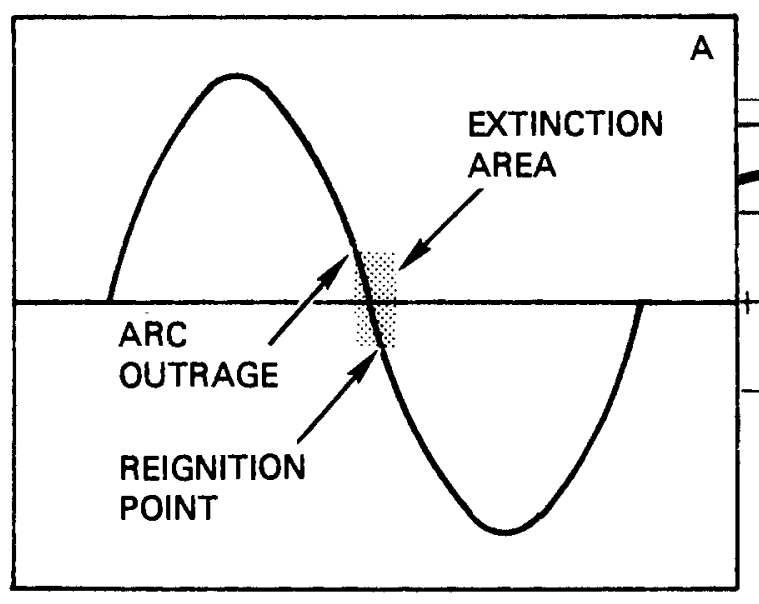

CONVENTIONAL SINE WAVE

"SQUARE" WAVE

FIGURES 102A \& B. Oscillograms of Sine Wave and Square Wave Alternating-Currents 152 
In Figure 102A, the schematic oscillogram of a sine wave of current, is shown the extinction area of the alternating-current arc when used in welding. As the arc current and voltage decrease, a point is reached at which there is not enough voltage to maintain the current with the electrons available and the arc goes out. The arc remains extinguished until the voltage available is high enough to restrike the arc with the electrons available.

To overcome the arc extinguishing-restrike problem, a square wave current, constant potential welding power supply was developed. Shown in Figure $102 \mathrm{~B}$ is an oscillogram of the square wave alternating-current taken with an oscilloscope. The transition time for switching from positive to negative or negative to positive current pulse is in the order of 80 to 150 microseconds. Thus the arc is reestablished with the opposite polarity before thermal electron emission decays to the point that the arc is difficult to restart and is unstable.

The advantages of square wave alternating-current submerged arc welding are:

- Since the welding current and thus the arc voltage are each balanced, the instantaneous heat input approaches that of direct-current.

- Any magnetic effects producing transverse arc blow, Page 35, are neutralized by the alternating-current and the arc remains central to the electrode across the grooves.

- Due to the fast reversing of polarities, the electron emission of the electrode wires and the weld puddle have very little time to decay and thus arc outages at polarity reversals are virtually eliminated.

- In normal direct-current submerged arc welding, the groove may be wide and have an angle of twenty degrees, Figure 103A153, while with square wave submerged arc welding the groove may be narrow and the angle may be as small as two degrees, Figure 103B 153 .

- The combination of all of the above reduces the filler metal, the number of passes, the amount of energy, and labor. 


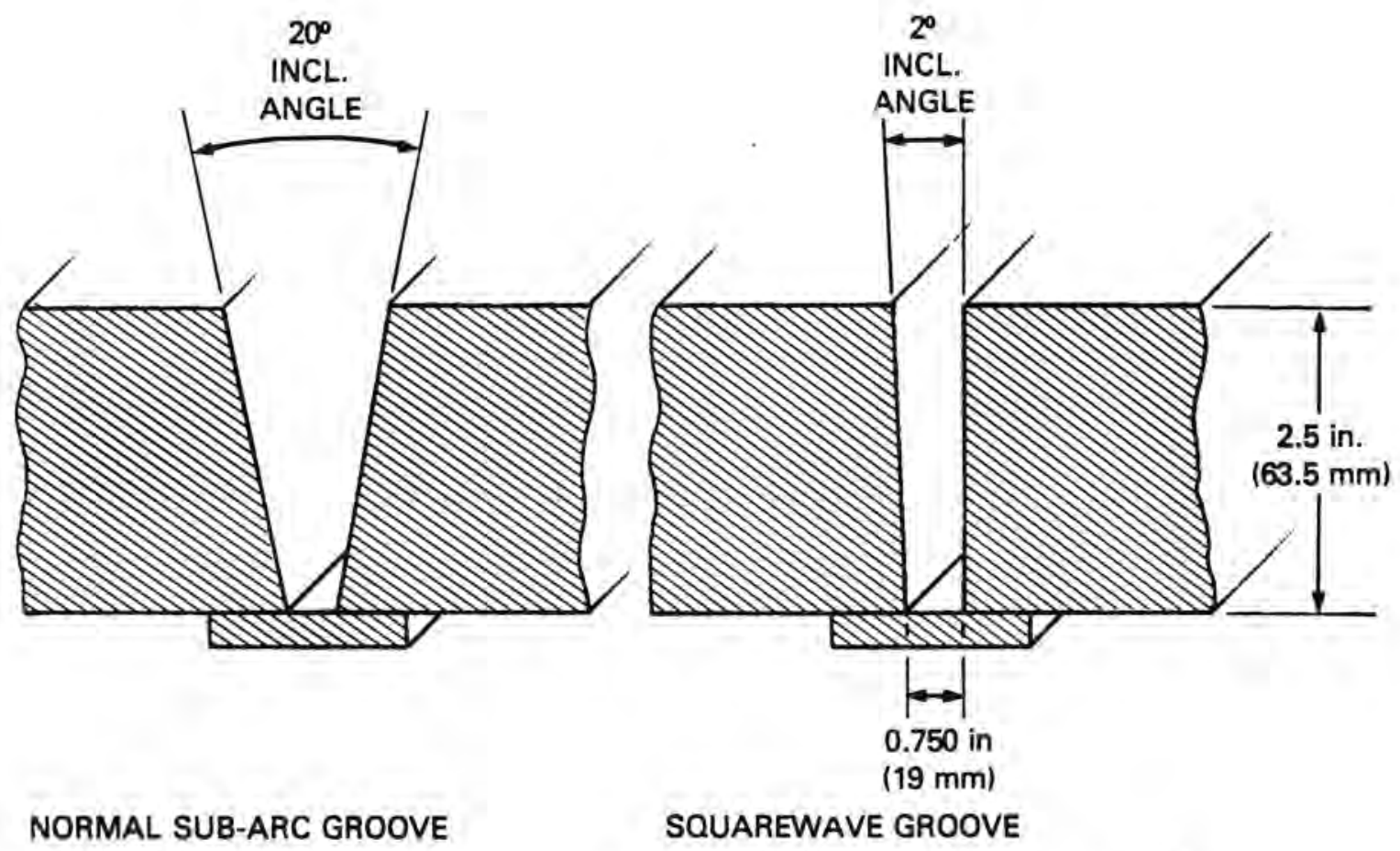

FIGURE 103A. Normal Submerged Arc Welding Groove 153 FIGURE 103B. Square Wave Submerged Arc Welding Groove 153

A recent advancement in square wave alternating-current submerged arc welding ${ }^{153}$ is the use of two electrodes. Several electrical systems, single-phase, two-phase, and three-phase, using two welding power supplies were investigated.

To the economics of narrow groove square wave alternating-current can be added the following significant economics when using two electrodes:

- Increased travel speed

- Increased deposition rates

- Produces stiffer or more stable arcs when compared to multi-electrode sine wave alternating-current arcs due to the constant value of current in each pulse.

The three-phase to two-phase transformer connection for power to two square wave building power supplies operating together is preferred because it balances the primary current in the three-phase supply lines. Two 
parallel connected square wave power supplies on a single-phase supply line or two on a three-phase open delta connection may be used when the unbalanced current in the three phase system is not of concern. 
PNL -4341

UC -25

\section{DISTRIBUTION}

\begin{tabular}{ll}
$\begin{array}{l}\text { No. of } \\
\text { Copies }\end{array}$ & $\begin{array}{l}\text { No. of } \\
\text { Copies }\end{array}$ \\
OFFSITE & OFFSITE \\
\hline 27 & DOE Technical Information Center \\
& Howard C. Ludwig \\
& 159 Roberta Drive \\
& Pittsburgh, PA 15221 \\
& John N. Kuhr \\
& Miller Electric Co. \\
& Appleton, WI 54912
\end{tabular}

R. G. Culbertson

Columbia Bas in College

Kennewick, WA 99301

Dr. R. R. Wright

BOC Limited

PO Box 222

Granby Employment Area

Milton Keynes MKI INN

England

D. R. Milner

University of Birmingham

Birmingham, England

W. J. Farrell

Sciaky Bros., Inc

4915 W. 67 St.

Chicago, IL 60638

E. B. LaVelle

825 South Hartford

Kennewick, WA 99336

American Welding Society

Po Box 351040

Miami, FL 33135

H. B. Carey

Hobart Brothers Co.

Troy, OH 45373
M. E. Gedguadas

Arc Machines, Inc.

11239 Ilex Ave.

Pacoima, Ca 91331

J. C. Needham

The Welding Institute

Abington Hall

Abington, Cambridge

CB1 6AL

England

George E. Linnert

The Welding Institute

PO Box 5268

Hilton Head Island, SC 29928

E. P. Vilkas

Astro Arc

Sun Valley, CA

R. K. Sager

1202 Marigold Drive

Albuquerque, NM 37122

Jay $B 1$ and

16924 S.W. 112 Court

Miami, FL 33151

Air Force Technical

Applications Center AFTAC/TNE

Attn: M. J. Kniedler

Patrick AFB, FL 32925

Air Force Weapons Laboratory

Att: Geogiana Hillyer

Technical Library

Kirtland AFB, NM 87117 


\section{DISTRIBUTION (cont'd.)}

No. of

Copies

OFFSITE (cont'd.)

Commanding Officer

Army Aberdeen Proving Ground

Att: Technical Library

Army Ballistics Labs

Bldg. 305 (Mrs. Frost)

Aberdeen Proving Ground, MD 210

Director, US Army Engineers

Att: Library

Waterways Experiment Station

PO Box 631

Vicksburg, MS 39108

Commanding Officer

Att: Stinfo Div DRDAR-TSS 59

US Army Armament Res. and Dev Comman.

Dover, NJ 07801

Army Tank Automotive Command

Att: DRDTA-J

US Army Tank Automotive R\&D

Command

Warren, MI 48090

Babcock \& Wilcox Co.

Att: Information Services

Nuclear Power Generation Dept.

PO Box 1260

Lynchburg, VA 24505

Bechtel Corp.

Att: Central Library

PO Box 3965

San Francisco, CA 94119

Westinghouse Elec. Corp.

Att: Librarian

Bettis Atomic Power Lab.

PO Box 79

West Mifflin, PA 15122
No. of

Copies

OFFSITE (cont'd.)

US Dept. of Agriculture

National Agricultural Library

Current Serial Record

Beltsville, MD 20705

US Dept. of Energy

Office of Basic Energy

Sciences ER-

Washington, DC 20545

US Dept. of Energy

Att: Chief/NE-50

Gas-Cooled Reactor Projects F309

Div. of Nuclear Res. and Applic.

Washington, DC 20545

US Dept. of Energy

Att: Deputy Assist. Secretary F0

Naval Reactor/NR-08

Div. of Naval Reactors

Washington, DC 20545

US Dept. of Energy

DOE Library/AD-453

MS G-043

Washington, DC 20545

US Dept. of Energy

Att: Asst. Mgr.

Energy Research \& Develop.

PO Box E

Oak Ridge, TN 37830

E. I. DuPont de Nemours \& Co.

Att: Document Custodian

Atomic Energy Division

Room 700, Wilm Trust

Wilmington, DE 19898

EG\&G Idaho, Inc.

Technical Library

PO Box 1625

Idaho Falls, ID 83415 


\section{DISTRIBUTION (cont'd.)}

No. of

Copies

OFFSITE (cont'd.)

Electric Power Research Institute Technical Library

Po Box 10412

Palo Alto, CA 94303

Energy Technology Engr. Center

Att: Librarian

Po Box 1449

Canoga Park, CA 91304

Garrett Corp.

Att: Library 93-45, T40

2525 West 190th St.

Torrance, CA 90509

General Electric Co.

Att: Librarian

Vallecitos Atomic Lab

PO Box 845

Pleasanton, CA 94566

General Electric Co.

Att: Division Librarian

Nuclear Energy Division (MC-328)

PO Box 1131

San Jose, CA 95108

Kent State University

Att: Prof. Richard Madey

Smith Laboratory of Physics

Kent, $\mathrm{OH} 44242$

Exchange and Gift Division

Library of Congress

10 First St., S.E.

Washington, DC 20540

Director, Atomic Energy Div

Office of the Chief of

Naval Operations

Dept. of the Navy

Washington, DC 20350
No.

Copies

OFFSITE (cont'd.)

Dept. of the Navy

Office of Naval Research

Code 422

Washington, DC 20360

Naval Ship Systems Command Hdq

Att: Librarian - Navships 08

Navy Dept.

Washington, DC 20360

Nevada Dept. of Energy

Att: Robt. R. Loux, Asst. Adm.

Energy Conservation \& Planning

1050 E. William, Suite 405

Carson City, NV 89710

US Nuclear Regulatory Comm.

Att: Director

Div. of Reactor Safety Research

Washington, DC 20555

US Nuclear Regulatory Comm.

Att: Library

Washington, DC 20555

Union Carbide Corp.

Central Research Library

Acquisitions Section

Oak Ridge National Lab

PO Box X

Oak Ridge, TN 37830

Southern Energy \& Environmental

Information Center

2300 Peachford Rd

One Exchange Place

Suite 1150

Atlanta, GA 30338

United Technologies Res. Center

Att: Library

400 Main St

East Hartford, CT 06108 


\section{DISTRIBUTION (cont'd.)}

No. of

Copies

OFFSITE (cont'd.)

Westinghouse Electric Corp.

Att: Library

Advanced Energy Systems Div.

PO Box 10864

Pittsburgh, PA 15236

ONSITE

DOE Richland Operations Office

H. E. Ransom

34 Pacific Northwest Laboratory

W. E. Anderson

D. G. Atteridge

R. A. Clark

T. B. Correy (15)

R. L. Dillon

C. R. Hann

A. J. Haverfield/W. R. Wiley

E. I. Husa

R. S. Kemper

R. F. Kle in

R. P. Marshall

R. E. Page

J. P. Pilger

Technical Information (5)

Publishing Coordination (2)

United Nuclear Company

L. P. Morgan

R. R. Studer

J. E. Tobin

Westinghouse Hanford Company

W. F. Brown 